\title{
Near-Field Optical Fluorescence Correlation Spectroscopy
}

\author{
Zur Erlangung des akademischen Grades eines \\ DOKTOR DES NATURWISSENSCHAFTEN \\ von der Fakultät für Physik des \\ Karlsruher Instituts für Technologie (KIT)
}

genehmigte

DISSERTATION

von

Dipl.-Phys. José Ronan Corentin Pérez aus Plømeur

Tag der mündlichen Prüfung:

03. 02.2012

Referent:

Priv. Doz. Dr. Andreas Naber

Korreferent:

Prof. Dr. Heinz Kalt 





\section{Contents}

1. Introduction 1

2. Theoretical and experimental aspects of near-field optics 5

2.1. Near-field optical microscopy . . . . . . . . . . . . . . . . . 5

2.1.1. Resolution enhancement . . . . . . . . . . . . 5

2.1.2. The aperture probe and the trade-off resolution/signal . . . 8

2.1.3. Single molecule imaging with triangular aperture probe . . . 13

2.2. Particuliar aspects of near-field optics . . . . . . . . . . . . . . . 14

2.2.1. Surface plasmon polaritons . . . . . . . . . . . . . . . . 14

2.2.2. From surface plasmon to nanoantennas . . . . . . . . . . . . 19

3. Principles of fluorescence correlation spectroscopy 21

3.1. Physics of fluorescence . . . . . . . . . . . . . . . . . 21

3.1.1. Fundamental aspects . . . . . . . . . . . . . . 21

3.1.2. The 3-level system . . . . . . . . . . . . . . . . . . 23

3.2. Fluorescence correlation spectroscopy . . . . . . . . . . . . . . . . . 28

3.2.1. Non-diffusing particles . . . . . . . . . . . . . . . . 28

3.2.2. Particles diffusing through a focussed Gaussian beam . . . . 35

4. Modeling of the optical near-field distribution at NSOM probes using FCS $\quad \mathbf{4 5}$

4.1. Principles of modeling . . . . . . . . . . . . . . . . . . . 45

4.1.1. Parameters of simulation . . . . . . . . . . . . . 45

4.1.2. Accuracy of the model . . . . . . . . . . . . . . . . 51

4.2. Modeling of the triangular aperture probe . . . . . . . . . . . 63

4.2.1. The Bethe-Bouwkamp model . . . . . . . . . . . . . 63

4.2.2. Light intensity distribution at a less idealized aperture . . . 66

4.2.3. FCS simulation at the triangular aperture probe . . . . . . 71

5. Experimental realization of near-field optical fluorescence correlation $\begin{array}{ll}\text { spectroscopy } & \mathbf{7 7}\end{array}$

5.1. Fabrication of the aperture probes and setup of the near-field optical microscope . . . . . . . . . . . . . . . 77

5.1.1. Probe fabrication . . . . . . . . . . . . . 77

5.1.2. Measurement setups . . . . . . . . . . . . . . . . 84

5.2. Analysis of the fluorescence signal . . . . . . . . . . . . . . . 88

5.2.1. Sample preparation . . . . . . . . . . . . . 88 
5.2.2. Auto- and cross-correlation calculation from the detected signals . . . . . . . . . . . . . . . . . .

6. Near-field optical microscopy combined with FCS: characteristics and applications

6.1. Comparison of the combination of NSOM probes with FCS over other

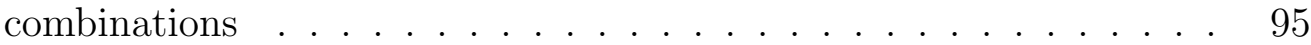

6.1.1. Time resolution . . . . . . . . . . . . . . . . . . . . 95

6.1.2. Excitation volume . . . . . . . . . . . . . . . . . . 98

6.1.3. Application domain and invasiveness . . . . . . . . . . 100

6.2. Application example of NSOM-FCS for the measurement of fast biological processes on the nanometer scale . . . . . . . . . . . . . 102

6.2.1. Imaging of single nuclear pores in a nuclear membrane . . . 102

6.2.2. Analysis of complex diffusion mechanism in the light of the confined diffusion model . . . . . . . . . . . . . . . . . 105

6.2.3. FCS measurement at a single nuclear pore . . . . . . . . 108

7. Detailed characterization of near-fields at an aperture probe with FCS113

7.1. Experimental aspects of NSOM-FCS measurements . . . . . . . 113

7.1.1. Stability of FCS measurements with a TA probe . . . . . . . 114

7.1.2. Reproducibility of NSOM-FCS measurements . . . . . . . . 114

7.1.3. Variability of NSOM-FCS with different TA probes . . . . . 119

7.2. Insight into the signal-to-noise ratio of NSOM-FCS . . . . . . . 122

7.2.1. Background origin of NSOM-FCS using gold coated TA probes 122

7.2.2. Probe aperture size dependent emission of background noise 124

7.2.3. Antenna effects at the triangular probe . . . . . . . . 126

7.3. Large intensity confinement at a TA probe . . . . . . . . . . . . 131

7.4. High time resolution of NSOM-FCS . . . . . . . . . . . . . 135

7.5. Change of the molecule's dynamic at the TA probe . . . . . . . 138

7.5.1. Effect of the solvent . . . . . . . . . . . . 138

7.5.2. Effect of the $\mathrm{pH} \ldots \ldots \ldots \ldots \ldots \ldots$

$\begin{array}{ll}\text { 8. Conclusion } & 147\end{array}$

A. Confined-diffusion model 149

$\begin{array}{ll}\text { Bibliography } & 151\end{array}$ 


\section{Introduction}

For now more than twenty years, optical methods are making use of the revolutionary ultra-high sensitivity for the detection of single molecule fluorescence at ambient conditions [1], [2]. Ever since, single molecule techniques were explored by a wide community of biologists, chemists and physicists with different aims.

The special field of biophysics, which aims at better understanding the function and structure of biological molecules, is particularly interested in a further development of the sensitivity and resolution of optical techniques. If electron microscopy techniques do help to gather useful information on these molecules, the preparation methods and observing conditions are often detrimental to their integrity [3]. Also, dynamic information is not accessible due to the fixation techniques needed to image samples in an electron microscope. A much more flexible way to gather this information is to use fluorescent molecules as a probe of the local environment of a biological structure. Due to high specificity of fluorescent markers, optical techniques have proven to be, until today, the best method to satisfy the needs of biophysicists regarding the observation of dynamic processes on a microscopic scale.

A single molecule technique is mainly characterized by two aspects which are closely related: its resolution capability and the achievable signal-to-noise ratio. But not only high-resolving imaging techniques allows one to observe single molecules. If the molecules to detect are sparse enough, lower-resolving techniques, such as wide field microscopy [1] or confocal microscopy [4], can also be used to detect single molecules. However, as the concentration of fluorescent molecules is increased, the resolution has to be increased too in order to further detect only single molecules. This requirement has particularly pushed the field of optical imaging forward and as a consequence a plethora of new imaging techniques were developed that increase the resolving capabilities at optical wavelength beyond the diffraction barrier. The first of these high-resolving techniques used to image single molecules was near-field scanning optical microscopy (NSOM) [5]. Many techniques have followed with the same goal of resolution enhancement but with different approaches. Some of these techniques aim at shaping the excitation light path to obtain a better resolution of a factor two or three [6], [7]. Many others are based on fluorescence switching techniques [8] [9] among which stimulated emission depletion (STED) [10], photoactivated localization microscopy (PALM) [11], stochastic optical reconstruction microscopy (STORM) [12] or its more flexible variant direct STORM (dSTORM) [13] are most prominent.

But resolution is not the only demanding criterion to detect single molecule at optical wavelengths. Also the fluorescence signal of the molecules of interest must 
be high enough to be able to compete with unwanted signals. These are commonly termed as background signal or noise and the signal-to-noise ratio (SNR) must be as high as possible. In the case of detecting fluorescence signals in a biologically relevant environment, the common noise sources are Rayleigh and Raman scattering or autofluorescence of biological samples. While many optical techniques can more or less reduce these background signals by, e.g., appropriate filter sets, time-gated fluorescence measurement [1] or working with larger excitation wavelength (for which autofluorescence is decreased) [14], their ability to increase the fluorescence signal is often limited. A young research field called plasmonics consists in designing metallic nanostructures at which surface charge density oscillations or surface plasmons are created [15]. These plasmons are coupled to light and enhance the excitation light or the fluorescence light intensity locally with a resulting fluorescence signal greater than that of conventional optical techniques [16], [17], [18].

Improving single molecule detection techniques is still needed but the reward is worth it. These techniques can gather information on many single molecules and this way allows one to measure the distribution of values for an experimental parameter instead of only its average value [19]. Also it removes the need for synchronisation of many molecules undergoing a time-dependent process [19]. For example, diffusion processes or binding kinetics can be monitored at thermodynamical equilibrium by detecting the fluorescence fluctuation of molecules diffusing in and out of a given excitation volume. In order to efficiently extract kinetic information from the large amount of data obtained by single molecule measurements, appropriate mathematical tools have to be used. Fluorescence Correlation Spectroscopy (FCS) [20] correlates the arrival times of subsequent photon detection events. The calculated correlated function depends on the kinetics of the process under study as well as on the particular shape of the excitation volume and provides most valuable information on the single molecule dynamics.

FCS was already combined with different high-resolving techniques. Using total internal reflection fluorescence (TIRF), surface bound dynamics could be measured [21]. Also STED was used in combination with FCS to show the principle reduction of the excitation volume [22]. But contribution to the fluorescence signal of molecules at the focal periphery seems to limit the use of this technique [22] [23]. So far, these techniques also lack a good time resolution and small excitation volumes.

The high intensity gradients at metallic nanostructures such as zero mode waveguides (ZMW) paved the way to highly temporally and spatially resolved FCS measurements [24]. However, methods using ZMW cannot simultaneaously image the structures at which dynamic processes are to be measured. Consequently, it is not possible to identify a specific structure and realize FCS measurements at this defined position.

As a scanning method, NSOM is able to give high-resolved images of sample surfaces. Though NSOM-FCS has already been applied to measurements of surface bound dynamics in biological environments [25] [26], its scanning ability was never used to target FCS measurements at a specific subwavelength structure. There are mainly two reasons for this. Firstly, imaging of biological substrates in water using 
feedback control is a complicated matter and requires the development of appropriate methods; secondly, conventional aperture probes cannot deliver a sufficient excitation intensity for the detection of ultra-low fluorescence at a high temporal resolution. Using the superior light throughput and light confinement at a triangular aperture probe, NSOM-FCS developed in this thesis could be used within this thesis for the first time to measure the translocation dynamics of proteins at a the subwavelength sized nuclear pores of a nuclear membrane.

A further major goal of this thesis was to derive a heuristical model for the correlation function of the free diffusion of fluorescent molecules at the triangular aperture probe and thereby laying a foundation for the understanding of the new method NSOM-FCS.

\section{Outline of this thesis}

Chapter 2 introduces the concept of near-fields and near-field aperture probes. Specifically it addresses the resolution enhancement and the trade-off between it and the excitation light intensity at the aperture probe and show single molecule measurements with this technique. Additionally, the concept of surface plasmon is explained along with its relation to the concept of nanoantenna. Fundamental aspects of fluorescence are presented in chapter 3. Then it is shown how FCS is used to gather kinectic information on the example of the different transition rates which govern the photophysical properties of fluorescent molecules. This will be then further applied to retrieve information on simple excitation volumes created by common optical techniques. Chapter 4 introduces the simulation model used to simulate fluorescence emission under defined conditions and particularly for the case of diffusion of fluorescent molecules in and out of the excitation volume created at the near-field aperture probe. After showing the accuracy of the simulation model for cases with known behavior, simulation results for different aperture probe models will be presented. In chapter 5, the experimental realization of NSOM-FCS is explained. This includes the near-field aperture probe fabrication process as well as details about the excitation and the detection setup used to measure FCS with a near-field aperture probe. Using the results of chapter 4, chapter 6 compares the NSOM-FCS techniques to other single molecule techniques combined with FCS. It also shows how the highly confined intensity at the near-field aperture probe can be used to measure the translocation of proteins at a biological membrane on a subwavelength scale. Finally, chapter 7 gives detailed results about the characterization of near-fields at an aperture probe. In this last chapter, the reproducibility of NSOM-FCS measurements is evaluated. Measurements with high SNR and extreme confined excitation volume will then be presented and attest to the quality of NSOM-FCS as a single molecule technique. This will be confirmed with FCS measurements at high concentration of fluorescent molecules and small time resolution.

A summary of the results and an outlook finally conclude the thesis. 



\section{Theoretical and experimental aspects of near-field optics}

\subsection{Near-field optical microscopy}

Near-field optics was first developed as a microscopy tool: Near-field optical microscopy has a long history and its origin is generally attributed to the Irish scientist Edward Hutchinson Synge. His first proposal in a personal communication with Einstein 1928 was to use the scattered light of an illuminated tiny gold sphere as a local probe on a sample of interest [27]. By illuminating a gold sphere and using it to raster scan the sample in close distance, he realized that features as small as the particle's size could be resolved [27]. This was the first step towards the development of higher resolving techniques than conventional optical microscopy and its diffraction barrier of typically $300 \mathrm{~nm}$. A few month later he published a slightly modified version where an aperture in a metal film, not a gold particle, would act as the local probe [28]. This version is the foundation of near-field optical microscopy using an aperture probe.

\subsubsection{Resolution enhancement}

Classical optical microscopy uses far-field optics and its resolution is therefore limited by the "diffraction barrier". A classical way of understanding how near-field optical microscopy breaks this barrier is to use the angular spectrum representation [29]. In this representation the fields are first Fourier transformed to extract the spectrum of spatial frequencies of the electromagnetic wave in a given plane (for example here the plane $z=0$ ).

$$
\mathbf{E}\left(k_{x}, k_{y}, z=0\right)=\iint_{-\infty}^{\infty} \mathbf{E}(x, y, z=0) \mathrm{e}^{-\mathrm{i}\left(k_{x} x+k_{y} y\right)} \mathrm{d} x \mathrm{~d} y
$$

with the transverse wave numbers $k_{x}$ and $k_{y}$.

As every other electromagnetic wave the evolution of $\mathbf{E}$ is governed by the wave equation (or Helmholtz equation) $\left(\Delta+\mathbf{k}^{2}\right) \mathbf{E}((r))=0$, where $|\mathbf{k}|=n 2 \pi / \lambda$ is the magnitude of the wavevector of the electric field $\mathbf{E}, \lambda$ the wavelength of $\mathbf{E}$ and $n$ the refractive index of the medium the field is propagating in. For example, for an electric field travelling in the positive $z$ direction, equation (2.1) in the Helmholtz 
equation gives the evolution of the spatial frequencies spectrum with distance $z$ :

$$
\mathbf{E}\left(k_{x}, k_{y}, z\right)=\mathbf{E}\left(k_{x}, k_{y}, z=0\right) \mathrm{e}^{-\mathrm{i} k_{z} z}
$$

where $k_{x}$ and $k_{y}$ and the longitudinal wavenumber $k_{z}$ are related to each other through $k_{x}^{2}+k_{y}^{2}+k_{z}^{2}=\mathbf{k}^{2}$. Because of the finite value of $|\mathbf{k}|$, we can find values of $k_{x}$ and $k_{y}$ for which $k_{x}^{2}+k_{y}^{2}>\mathbf{k}^{2}$. For these values $k_{z}$ becomes imaginary and the amplitude of the corresponding spectral components $\mathbf{E}\left(k_{x}, k_{y}\right)$ decays exponentially with $z$. In the case of classical microscopy, the image is obtained by measuring $|\mathbf{E}|^{2}$ in the far-field where $z \gg \lambda\left(k_{z} z \gg 1\right)$. Correspondingly, many spectral components vanish and cannot contribute anymore to the image. For an infinitely thin slit illuminated in the object plane and centered at $x_{0}$, which corresponds to a simple one dimensional case, the spectral components are homogeneously distributed over the spatial frequencies $k_{x}$ as $\mathrm{e}^{-\mathrm{i} k_{x} x_{0}}$. Consequently, in the image plane [30]:

$$
E_{i}(x, y, z) \propto \int_{-k_{\max }}^{+k_{\max }} \mathrm{e}^{\mathrm{i} k_{x} x_{0}} \mathrm{e}^{-\mathrm{i} k_{x} x} \mathrm{~d} k_{x}=2 \frac{\sin \left(k_{\max }\left(x-x_{0}\right)\right)}{\left(x-x_{0}\right)}
$$

where $k_{\max }=n \frac{\omega}{c}$. Above this value of $k_{x}$, the spectral components $E\left(k_{x}\right) \operatorname{grad}-$ ually vanish because of the afore mentioned exponential decay. $n$ is the index of refraction of the propagating medium, $\omega$ the light frequency and $c$ the vacuum speed of light. It means it is not necessary to take the spectral frequencies $k_{x}>k_{\max }$ into account in the integral. Due to this decay, these spectral components are called evanescent waves in contrast to propagating waves $\left(E\left(k_{x}\right)\right.$ for $\left.k_{x} \leq k_{\max }\right)$. The result is not a point source as in the object plane but a spot of finite width, given by the distance of the first minima on each side of the main maximum (see figure 2.1):

$$
\Delta x=\frac{\pi}{k_{\max }}=\frac{\lambda}{2 \mathrm{NA}}
$$

where $\mathrm{NA}=n \sin \theta$ is the numerical aperture of the objective responsible for the image formation. This result is the Rayleigh criterion for the resolving power of the objective and also the consequence of the so-called "diffraction barrier". As shown in [30], it is a direct consequence of the Heisenberg uncertainty principle: only the photons with a wavenumber $k_{x}<\mathrm{NA} \frac{\omega}{c}$ can reach the image plane (at most $k_{x}<n \frac{\omega}{c}$ in the ideal case where all the photons propagating in the half space in direction of the image plane can be collected). The uncertainty on the photon wavenumber is therefore at most $\Delta k_{x}=2 \mathrm{NA} \frac{\omega}{c}$. As the uncertainty limit on the position of the photon in the image plane $\Delta x$ is given by $\Delta x \Delta k_{x}=2 \pi$, the resolution $\Delta x$ cannot be better as the Rayleigh criterion. A possibility to increase the resolution and consequently the bandwidth $\Delta k_{x}$ is therefore to gather spectral components with $k_{x}>k_{\max }$. This is what is done in conventional far-field microscopy when a sample is imaged in an immersion medium: the medium with a higher refraction index than air (typically liquids such as oil with $n \sim 1.5$ or water with $n \sim 1.3$ ) propagate higher spectral components $E\left(k_{x}\right)$. The resolution enhancement is of 

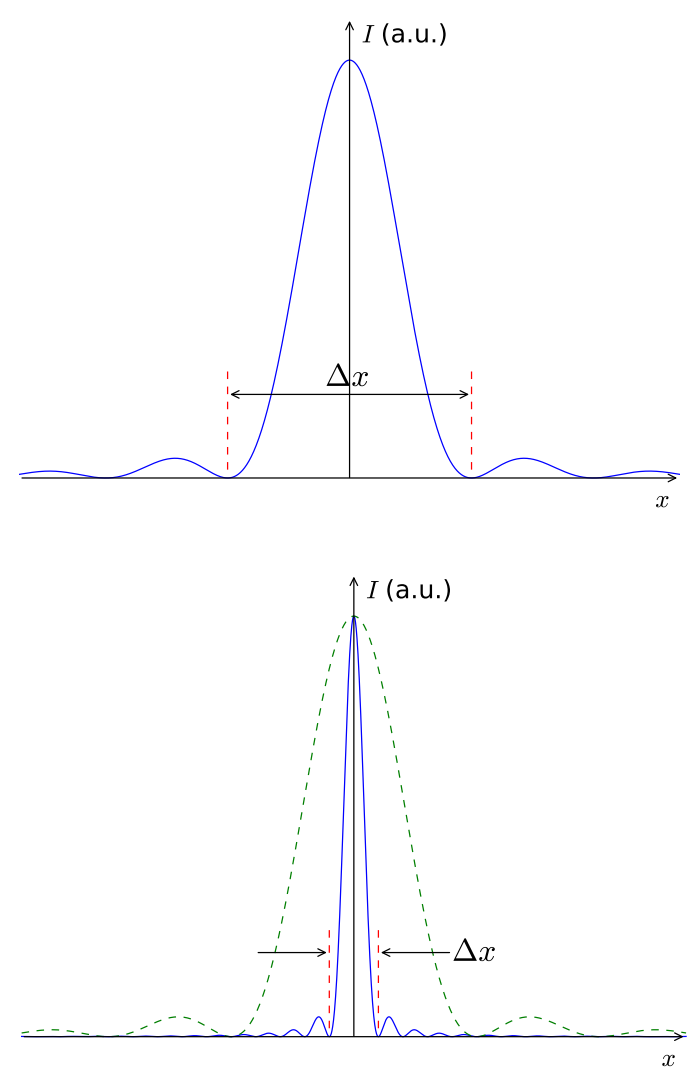

Figure 2.1.: Left: Diffraction limited spot in the image plane of an infinitely thin slit illuminated in the object plane. The width of the spot is given by $\Delta x=\frac{\pi}{k_{\max }}$ with $k_{\max }$ being the maximum spatial frequency $k_{x}$ for which the spectral component $E\left(k_{x}\right)$ can propagate in the far-field. Right: If spectral components with higher spatial frequencies take part in the image formation process, then the image is sharper. That is what near-field optics achieves. 
factor $n$, which is usually not better than 1.5 with liquids. A solid immersion lens can further increase the resolution by imaging through a solid with higher refractive index but the enhancement is hardly doubled compared to the Rayleigh criterion [31].

The goal of near-field optical microscopy is also to gather spectral components with higher spatial frequencies as $k_{\max }$ but in a slightly other manner: the original idea of E.H. Synge was to place an object smaller than the structure to image at a distance $z \ll \lambda$ where the evanescent waves have not yet vanished. This idea relies on the reciprocity theorem in optics: if a small object illuminated by a propagating wave generates evanescent waves then a small object should also be able to convert evanescent waves to a propagating wave. This relation is shown theoretically in [30] in terms of spectral components. A spectral component with a given spatial frequency $K_{x}>k_{\max }$ of the object to image is diffracted at a small object (smaller than the object to image, itself smaller than the smallest distance resolvable from the Rayleigh criterion). The small diffracting object then transforms this spectral component to a propagating wave that can reach the image plane. As a result, the image obtained by scanning the object over the sample contains a wider spectrum of spatial frequencies and the image is sharper, higher resolved (see figure 2.1). The smaller the object, the wider the band of spatial frequencies the object is able to scatter in the far-field. Consequently a smaller object will create an image of the sample with higher resolution.

However, near-field optical microscopy cannot be simplified to a technique allowing a larger bandwidth of spatial frequencies in the imaging process. Such a scheme would totally miss the description of resonant effects between exciting field and probe (field enhancement) or probe and sample (emission enhancement). These effects are grouped under the denomination "antenna effects" and will be described in section 2.2 .

A direct consequence of imaging the near-field distribution of a sample is that the scattering object has to be brought a few nanometers close to the sample. This is usually achieved by different force-distance control schemes, the most known of them is the shear force distance control [29]. This aspect is of uttermost importance in near-field optical microscopy to prevent the probe object from crashing onto a sample with a hard surface. In this work, however, no such samples were investigated and as a consequence an implementation of a force-distance control was not required.

\subsubsection{The aperture probe and the trade-off resolution/signal}

The first experimental realizations of near-field optical microscopy used metal coated structures with small aperture as near-field optical probe [32]. Although there have been many variations of this kind of microscopy such as the apertureless based technique [33], [34] or the aperture based technique in collection mode, also knwon as photon scanning tunneling microscopy (PSTM) and scanning tunneling optical microscopy (STOM) [35], [36] we will mainly deal with aperture based 
technique in illumination mode as it is the kind of microscopy used in this work.

In the aperture based near-field optical microscopy, the subwavelength sized object responsible for scattering spectral components with high spatial frequencies is usually the apex of a glass tip that is coated with a metal everywhere but at the apex. The typical size of the uncoated glass aperture extends from a few $10 \mathrm{~nm}$ to above $100 \mathrm{~nm}$. Even in this kind of technique there are different variations that are characterized by the probe fabrication and the aperture creation process. Though the first measurements with aperture probe used an etched quartz crystal coated with aluminum [32], thermally pulled fiber very soon became the standard [37],[38]. Not as widespread as the thermally pulled or the etched probe, the triangular aperture probe [39] is another important member of the family of aperture probes.

Although this work uses triangular aperture probes, the following cannot be directly applied to them, however, it allows to get a clear picture of the main drawback of aperture probes: the weak intensity throughput and the necessary trade-off between high resolution and still high enough signal-to-noise ratio (SNR).

By using aperture probes the exciting light is usually launched into a metal coated piece of glass, which acts as a metallic cylindrical waveguide filled with a dielectric. Contrary to the ideal plane wave which is extended in the whole transverse plane, the electromagnetic wave in a waveguide is constrained. As a result the transverse wavenumbers $k_{x}$ and $k_{y}$ take finite values. These values define the different TE (transverse electric) and TM (transverse magnetic) modes, i.e. modes that light can couple to and with their electric and magnetic fields, respectively, solely in the transverse plane [40]. The number of available modes increases with the size of the cross-section of the waveguide. Furthermore, in the simplified case of a coating with a perfect conductor and for a large cross-section of the waveguide, the longitudinal wavenumber $k_{z}$ (also called propagation constant in waveguide theory) is purely real: the electromagnetic wave propagates in the waveguide without energy lost. But in aperture probes the cross-section of the waveguide is bound to decrease down to the aperture area. As it does, less and less modes are available for propagation. Ultimately a certain value for the dimension of the waveguide in the transverse plane is reached and the last mode disappears. The longitudinal wavenumber $k_{z}$ of the electromagnetic wave becomes purely imaginary and the wave becomes evanescent. This region of the probe, over which only evanescent waves can exist, is called the cut-off region and is depicted in figure 2.2. The longer the cut-off region (i.e. the longer the extension of the cut-off region in the $z$ direction), the less light can reach the end of the cut-off region. In the case where the coating metal is ideal, most of the light is reflected. Only the tiny fraction that made its way to the aperture is missing in the reflected light. This process is similar to the case of frustrated total internal reflection where a tiny fraction of the light impinging on an interface above the critical angle traverses the gap between two high refraction index material to further propagate, leading to a smaller reflection coefficient of the interface. For real metals some part of the light that does not reach the aperture is absorbed in the coating instead of being reflected. The fraction of the light intensity dissipated 
in this way is usually as high as two third of the incoming intensity [29].

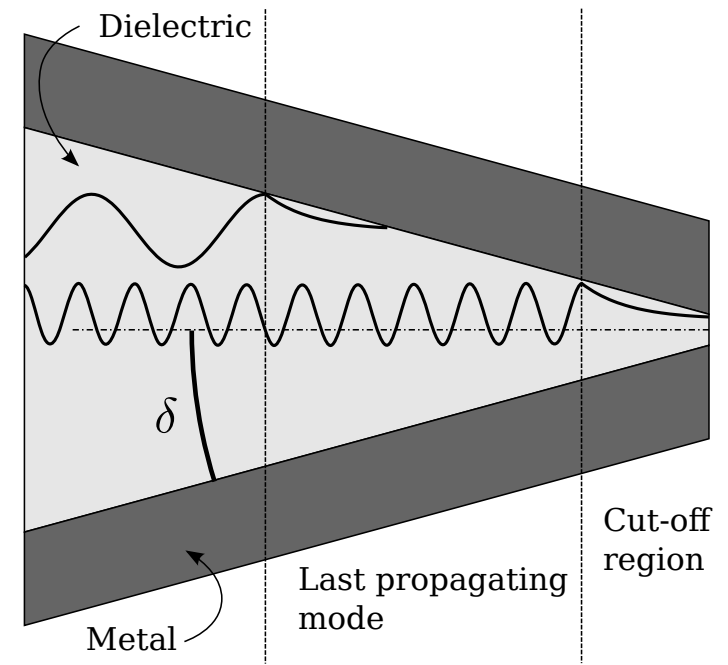

Figure 2.2.: Illustration of the damping of 2 different modes in an aperture fiber probe with diminishing cross-section. At a certain point only one mode still exists. If the cross-section of the waveguide further diminishes, the light cannot propagate anymore and the electromagnetic wave becomes evanescent. The longer this cut-off region, the less energy is transmitted through the aperture. $\delta$ is the taper angle of the fiber probe. Adapted from [41].

It was shown in the previous section that the smaller the scattering object the higher the resolution of the image. In the case of the aperture probe, decreasing the size of the scattering object means decreasing the aperture size and consequently increasing the cut-off region. Therefore a smaller aperture will provide a better resolution but at the cost of the light intensity that excites the sample. Fluorescence (see section (3.1)), that is generally excited at the sample, has already a small absorption cross-section. If the excitation intensity is too small then the fluorescence intensity falls under the noise level of the detectors and no image can be reconstructed. Therefore aperture based near-field optical microscopy always has to deal with the trade-off between resolution and signal. For fiber aperture probes it is the reason why the diameter of the aperture is mostly chosen not smaller than $50 \mathrm{~nm}$ to $100 \mathrm{~nm}[29]$.

However, figure 2.2 shows that the size of the cut-off region can be controlled by the taper angle $\delta$ of the aperture probe. This is actually the critical parameter in aperture probe fabrication and a lot of effort has been invested to increase it as much as possible [42], [43], [44]. Simulations of the light throughput at an aluminum coated dielectric waveguide show for example that changing the taper angle from $10^{\circ}$ (typical for thermally pulled fiber probe, [45] to $45^{\circ}$ increases the power throughput by eight orders of magnitude (figure 2.3). Even though chemical etching of probes enables one to produce probes of a given taper angle in a relatively reproducible 
way, the glass surface is still very irregular after the etching process, which impairs the subsequent metallization of the probe: pinholes and imperfections in the metal coating interfere with the near-field at the aperture probe and lead to an optical signal that is more difficult to interpret [41]. Though thermally pulled fiber probes have a small taper angle they do not suffer from coating artifacts as the glass surface gets very smooth upon pulling [41].

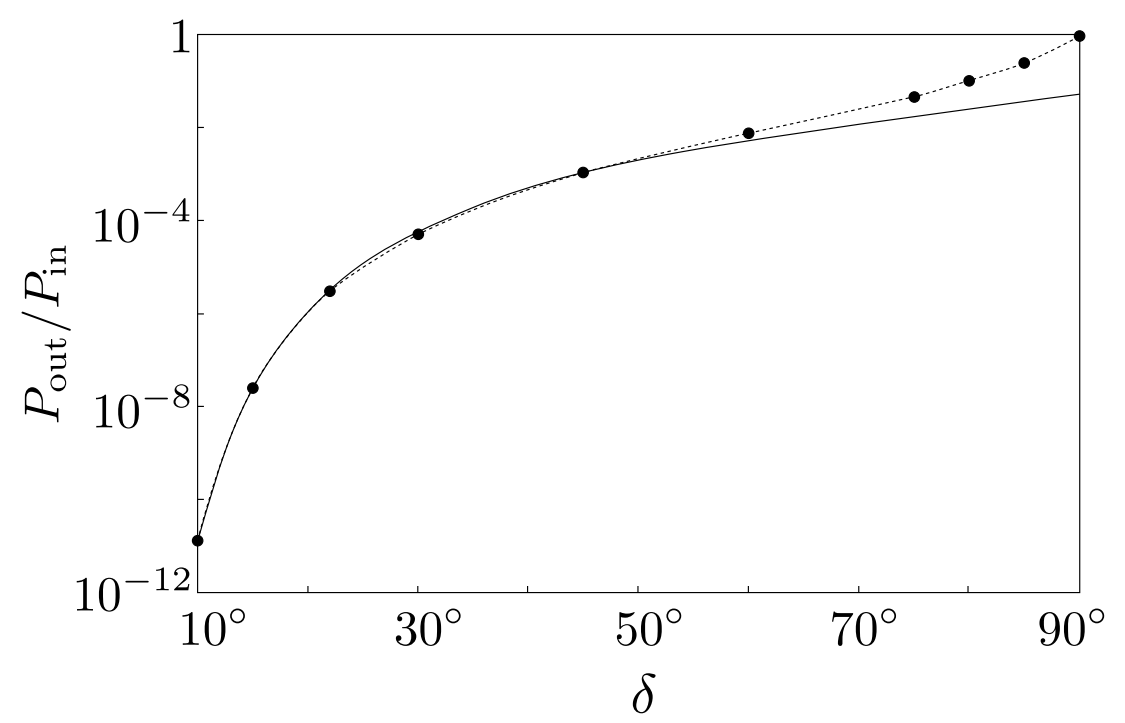

Figure 2.3.: Power transmission of an aperture probe as a function of the taper angle $\delta$. The aperture diameter is $20 \mathrm{~nm}$ and the wavelength $\lambda=488 \mathrm{~nm}$. The points correspond to a three-dimensional computation whereas the solid line is computed with the mode matching approximation [29]. This approximation fails to describe power transmission correctly at taper angles above $50^{\circ}$ due to neglected reflection. Adapted from [29].

The triangular aperture probes that are used in this work have the advantage of a high taper angle [39]. Even though very high taper angle $\delta>45^{\circ}$ can be achieved with this technique, it is not useful to do so. This is due to the finite skin depth of metals at optical wavelength: at a very large taper angle the metal coating is very thin at the aperture and the electromagnetic wave can propagate through it with nearly no attenuation. The effect is a loss of confinement of light intensity at the aperture probe. The leakage of the fields at the sidewalls of the aperture leads to a larger apparent size of the aperture and results in a loss of resolution (see figure 2.4). For a given aperture size, the taper angle of $45^{\circ}$ chosen for this work, is therefore a trade-off between maximum intensity throughput and small field leakage in the metal coating.

In the first section, it was shown that a small object like the aperture of a near-field optical probe is characterized by the ability to scatter a broader spectral frequency range in the far-field with all the more efficiency when this object is small compared to the structure to image. However, this high resolution imaging takes 


\section{a}

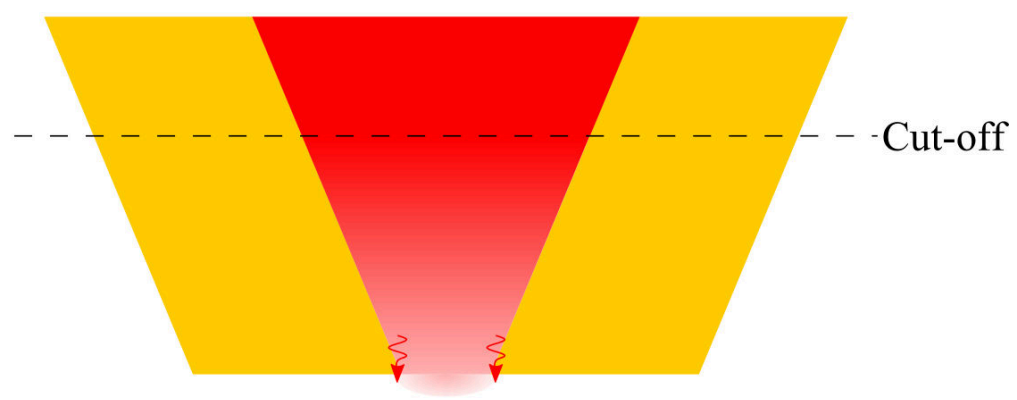

b

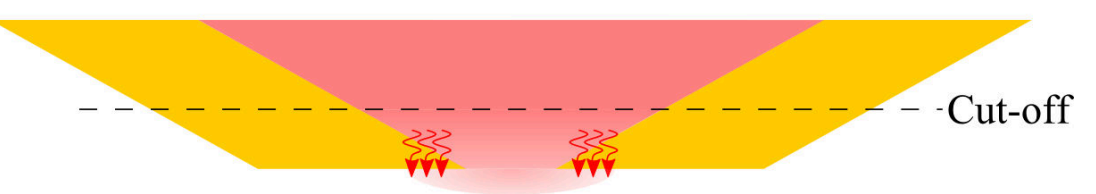

Figure 2.4.: Increasing the taper angle of the aperture probe leads to a smaller cutoff region. Therefore, for a given aperture size, less light intensity is required to achieve a given intensity in the near-field of the aperture when the taper angle is increased (intensity represented by the intensity of the red color). However, this also has adverse consequences: due to the finite skin depth of the metal coating, electromagnetic fields leak through the coating and if the taper angle is too large the region around the aperture contribute to a greater extent to the near-field distribution and thus impairs the achievable resolution. 
place at the cost of the excitation intensity at the aperture: light runs towards the aperture in the cut-off region and becomes evanescent. Additionally, the smaller the taper angle of the probe the less intensity is available at the output of the probe. This is particularly an issue for thermally pulled fiber probes where the aperture angle is small due to the fabrication process, which requires to fabricate these probes with large aperture size at the cost of the achievable resolution. Cut-off is also an issue for etched fiber probes as the rough glass surface caused by etching, results in an inhomogeneous and rough coating, which leads to field leakage through the coating and interference with the near-field at the aperture [41], [29].

Near-field optical microscopy with the triangular aperture probes, that are used in this work, do not suffer to such an extent from these drawbacks and can be consequently used to image single molecules at a very high resolution as shown in the next section.

\subsubsection{Single molecule imaging with triangular aperture probe}

In order to demonstrate the high SNR offered by a near-field aperture probe with triangular aperture and consequently the viability of the technique to measure single molecule processes, these probes were used to scan a sample made out of terrylenediimide (TDI) molecules embedded in a $10 \mathrm{~nm}$ thick polymethylmetacrylate (PMMA) film $[46]^{1}$. An example of a scan using a triangular aperture of a size of $50 \mathrm{~nm}$ and coated with aluminum is given in figure 2.5. The TDI molecules are recognizable as small spots with different shapes and intensities, which can be well explained by the fact that the dipole moment of the TDI molecules are randomly oriented in the PMMA film and also at distances fluctuating wihtin $10 \mathrm{~nm}$ (the thickness of the film) to the aperture. Figure 2.6 shows the high resolution achievable with the triangular aperture in some example fluorescence spots. For these spots, a very high SNR can be seen and a resolution down to $30 \mathrm{~nm}$ is obtained.

Using a numerical model for the interaction of a dipole with a triangular aperture in an aluminum film, the spot shapes could be explained as a result of the local interaction of the electric field with the dipole orientation of the TDI molecules. This was also shown to be consistent with both polarization perpendicular and parallel to a rim of the triangle.

Additionally, the high resolution enhancement at the triangular aperture probe compared to the usual thermally fiber pulled and chemically etched probes with circular aperture results from the localization of the electric fields at one rim of the triangular aperture for polarization perpendicular to this rim, whereas the circular aperture shows two spots of high electric field values on both side of the aperture along the polarization axis.

With these promising results regarding SNR and resolution, near-field aperture probes with triangular aperture are best suited to probe the dynamic of single

\footnotetext{
${ }^{1}$ For this imaging process, a control force distance was necessary and is explained in the given reference
} 


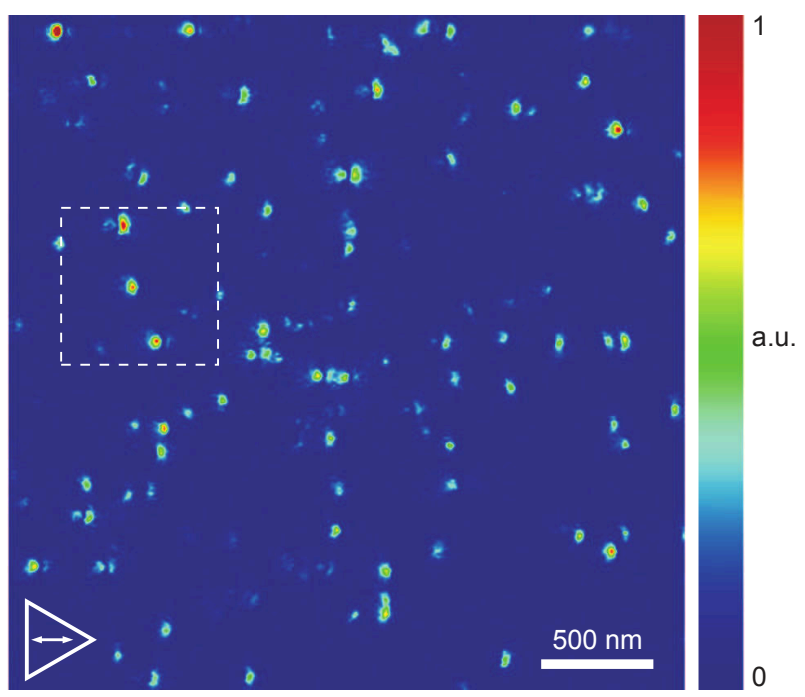

Figure 2.5.: Imaging of TDI molecules embedded in a $10 \mathrm{~nm}$ thick PMMA film using a $50 \mathrm{~nm}$ sized and aluminum coated triangular aperture probe (represented by the white triangle). The orientation of the arrow represents the polarization of the exciting light (adapted from [46]).

molecules at the nanometer scale. This feature made it possible to use this kind of probe for use with fluorescence correlation spectroscopy (FCS) for the first time.

In the work on TDI molecules embedded in a PMMA matrix, the fluorescent molecules were bound to stay at a finite distance to the metal coating of the probe. However, for FCS measurements on fluorescent molecules, that are free to diffuse around the aperture, the distance between molecules and metal coating at the aperture can become arbitrarily small so that additional effects influence the fluorescence signal that is emitted. The most known of these effects are fluorescence quenching and fluorescence enhancement. This last point was already shown to result from electron oscillations at a metal dielectric interface that are known as surface plasmons [47], [48], [49]. The following section introduces that and shows how it can affect the near-field at the aperture probe.

\subsection{Particuliar aspects of near-field optics}

\subsubsection{Surface plasmon polaritons}

Surface plasmons are charge density waves caused by a collective oscillation of electrons at a metal surface. The term polariton is used to describe the coupling between this charge density wave and a photon. The origin of surface plasmon polaritons (SPP) can be entirely derived from Maxwell's equations applied on an electromagnetic wave impinging on the interface between two media. Starting from the Helmholtz equation $\left(\Delta+\mathbf{k}^{2}\right) \mathbf{E}((r))=0$, it appears that a p-polarized plane 

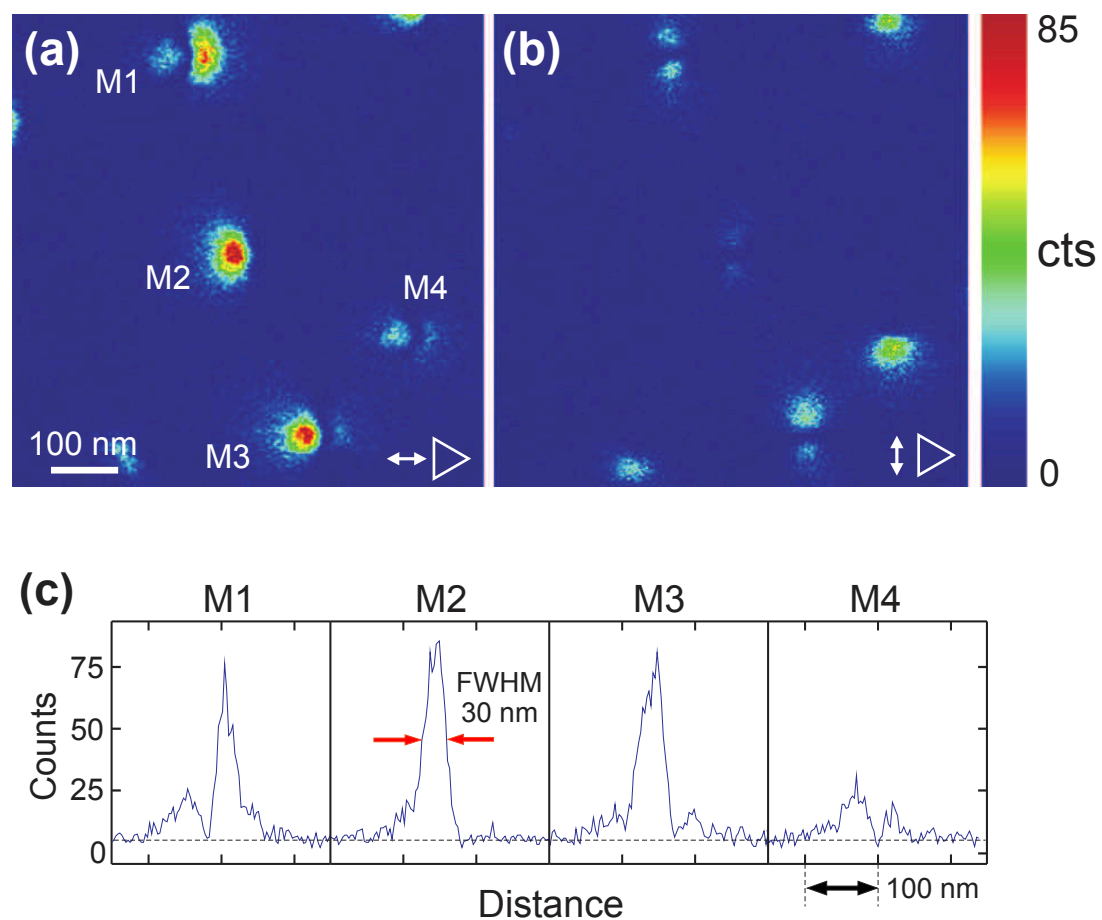

Figure 2.6.: Enlarged view of the framed area in figure 2.5 for two different polarizations of the exciting light (indicated by the white arrows). The different shapes of the fluorescence spots are caused by different orientations of the molecules embedded in the PMMA film. The cross-sections of the spots tagged in the enlarged view show that the triangular aperture features typically a resolution of $30 \mathrm{~nm}$ (adapted from [46]). 
wave is the only solution that fulfills this and can sustain a plasmon at the interface. Although a s-polarized wave also fulfills the Helmholtz equation, a SPP cannot be launched from this wave at an interface as its electric field has no normal component to the interface. Letting $z=0$ be the interface, the impinging p-polarized plane wave can be written as follows:

$$
\mathbf{E}_{j}=\left(\begin{array}{c}
E_{j, x} \\
0 \\
E_{j, z}
\end{array}\right) \mathrm{e}^{\mathrm{i}\left(k_{x} x-\omega t\right)} \mathrm{e}^{\mathrm{i} k_{j, z} z} \quad(j=\{1,2\})
$$

where $\mathrm{j}$ designates the medium in which the wave is propagating. $k_{x}$ does not need an index as the parallel component of the wavevector is conserved at the interface.

Then applying the Maxwell's equation $\nabla \cdot \mathbf{D}=0$ separately in the two different media and fulfilling the boundary conditions for the tangential component of $\mathbf{E}$ and the normal component of $\mathbf{D}$ leads to the following system of equations:

$$
\begin{aligned}
k_{x} E_{1, x}+k_{1, z} E_{1, z} & =0 \\
k_{x} E_{1, x}+k_{2, z} E_{2, z} & =0 \\
E_{1, x}-E_{2, x} & =0 \\
\epsilon_{1} E_{1, z}+\epsilon_{2} E_{2, z} & =0
\end{aligned}
$$

where $\epsilon_{1}$ and $\epsilon_{2}$ are the dielectric constants of each medium and depend on the light frequency: $\epsilon_{j}(\omega)$. This system can be written as a matrix:

$$
\left(\begin{array}{cccc}
k_{x} & k_{1, z} & 0 & 0 \\
k_{x} & 0 & 0 & k_{2, z} \\
1 & 0 & -1 & 0 \\
0 & \epsilon_{1} & 0 & \epsilon_{2}
\end{array}\right)\left(\begin{array}{l}
E_{1, x} \\
E_{1, z} \\
E_{2, x} \\
E_{2, z}
\end{array}\right)=\left(\begin{array}{l}
0 \\
0 \\
0 \\
0
\end{array}\right)
$$

which has a solution if the determinant of the left matrix vanishes. The only nontrivial solution is:

$$
k_{x}^{2}=\frac{\epsilon_{1} \epsilon_{2}}{\epsilon_{1}+\epsilon_{2}} k^{2}
$$

where $k$ is the vacuum wavevector. As the wavevector in each medium fulfills $k_{j}^{2}=\epsilon_{j}^{2} k^{2}=k_{x}^{2}+k_{j, z}^{2}$, it follows:

$$
k_{j, z}^{2}=\frac{\epsilon_{j}^{2}}{\epsilon_{1}+\epsilon_{2}} k^{2} \quad(j=\{1,2\})
$$

From these two last equations we can see that for a wave propagating on the interface $\left(\operatorname{Re}\left(k_{x}\right)>0\right)$ and decaying in both two media $\left(\operatorname{Im}\left(k_{j, z}\right)>0\right)$ to exist, following conditions have to be fulfilled: 


$$
\begin{array}{r}
\epsilon_{1}(\omega) \cdot \epsilon_{2}(\omega)<0 \\
\epsilon_{1}(\omega)+\epsilon_{2}(\omega)<0
\end{array}
$$

These two conditions would be fulfilled for an ideal metal (for example $\epsilon_{1} \rightarrow$ $-\infty)$ interfacing with vacuum $\left(\epsilon_{2}=1\right)$ but as light cannot exist in ideal metals $k_{1, z}^{2} \rightarrow-\infty \Rightarrow \mathbf{E}_{1} \rightarrow 0$, the plasmon phenomenon can only occur at interfaces with real metals. These metals are always lossy $\left(\operatorname{Im}\left(\epsilon_{1}\right)>0\right)$ and the conduction electrons cannot instantly follow the electric fields because of their finite mass and their scattering at the lattice $\left(\operatorname{Re}\left(\epsilon_{1}\right)\right.$ is finite). For noble metals with large negative real part of their dielectric constant like gold $\left(\operatorname{Re}\left(\epsilon_{1}(\omega)\right)=-13.2\right.$ at $633 \mathrm{~nm}$ [50]) at the interface with vacuum or even glass $\left(\epsilon_{2} \simeq 2.25\right.$ at $\left.633 \mathrm{~nm}\right)$, this condition is fulfilled.

All characteristics of a SPP at a planar interface between gold $(\tilde{\epsilon}=-13.2+\mathrm{i} 1.08)$ and glass $\left(\epsilon_{2} \simeq 2.25\right)$ or gold and water $\left(\epsilon_{2} \simeq 1.77\right.$, [51] $)$ at $\lambda=633 \mathrm{~nm}$ can then be calculated. These data are referenced in table 2.1 .

\begin{tabular}{|l|c|c|}
\hline Interface & Gold/Glass & Gold/Water \\
\hline Wavelength & $385 \mathrm{~nm}$ & $443 \mathrm{~nm}$ \\
Propagation length & $3.7 \mu \mathrm{m}$ & $5.6 \mu \mathrm{m}$ \\
Decay length in the metal & $13 \mathrm{~nm}$ & $13 \mathrm{~nm}$ \\
Decay length in the dielectric & $74 \mathrm{~nm}$ & $97 \mathrm{~nm}$ \\
\hline
\end{tabular}

Table 2.1.: Characteristic values of a SPP at a planar interface between gold and glass or gold and water. The SPP wavelength was computed with $\frac{2 \pi}{\operatorname{Re}\left(k_{x}\right)}$ and its propagation length (distance at which its intensity falls to 1/e) along the interface metal/dielectric with $\frac{1}{2 \operatorname{Im}\left(k_{x}\right)}$, where $k_{x}$ is given by equation 2.12. The decay length in the metal or in the dielectric was calculated through $\frac{1}{2 \operatorname{Im}\left(k_{j, z}\right)}$ with $k_{j, z}$ from equation 2.13 .

A SPP cannot be excited from a free space photon at the first coming interface because at any frequency and angle their wavevector component in propagation direction of the plasmon has a higher amplitude than the wavevector of a free space photon. Therefore energy conservation and impulse conservation cannot be satisfied simultaneously. To excite a SPP using optical wavelength three main methods are known. First, using the frustrated reflection of light at an interface [52], [53]. In this case, light impinges on a metal interface through a medium with high refractive index like glass where it is reflected. Evanescent waves are created at the interface glass/metal. However, the metal layer is kept thin and adjacent to another dielectric of lower refractive index than glass (for example water). The high impulse photon in the evanescent field can thereby excite SPP at the metal/water interface (see figure 2.7). Another technique to excite SPPs consists in using a grating coupler where the additional momentum is given by a periodic metallic structure to ensure 
impulse conservation [54]. The third method is intimately linked to near-field optics as it uses the large spectrum of spatial frequencies created by scattering of light at a subwavelength particle [55]. When the planar interface is in the near-field of the particle and their distance is small enough, photons with high impulse are available to launch a SPP in the interface.

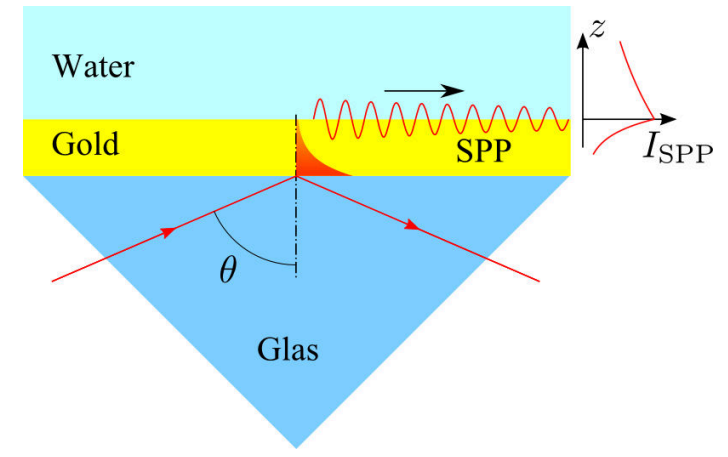

Figure 2.7.: Illustration of the SPP excitation in the Kretschmann configuration. Light impinges at a defined angle through a glass prism on a gold layer. Since the electromagnetic wave cannot propagate in the gold layer only evanescent waves exist there, the intensity of which decreases exponentially with gold thickness. If the metallic layer is thin enough, a SPP is created at the surface gold/water. It propagates along the interface and eventually vanishes. Perpendicular to the interface the electric field of the SPP decays much quicker in gold than in water (see schematic representation of $I_{\mathrm{SPP}}$ in $z$ direction on the right). The penetration depth of the intensity of a plane wave reflected at the surface and that of a SPP in gold is comparable (see table 2.1): about $13 \mathrm{~nm}$ for $\lambda=633 \mathrm{~nm}$, i.e. $\sim \lambda / 50$. The decay length of the intensity of the SPP in water is about $\sim \lambda / 6.5$ and the propagation length of the SPP at the interface is with $\sim 9 \lambda$ much larger than the decay in the dielectric. In this configuration the angle $\theta$ at which the SPP is created is $\theta \sim 72.3^{\circ}$. For comparison $\theta \sim 43.9^{\circ}$ at a gold/air interface.

SPPs have been used for years because of the extreme sensibility of the resonance condition (value of the angle $\theta$ ) for the creation of a SPP at an interface. Calculations at a silver-air interface separated by a layer of water as thin as $3 \mathrm{~nm}$ shows that $\theta$ is shifted by $0.2^{\circ}[29]$. This high sensitivity stems from the enhanced intensity directly at the interface metal-dielectric compared to the intensity of the radiation that is used to excite the SPP. At a gold-air interface for example, an enhancement factor of 10 can be achieved when exciting the SPP at $633 \mathrm{~nm}$ through a $50 \mathrm{~nm}$ thick gold film [29].

This section gave a general insight on SPPs, i.e. electron density wave propagating on a two dimensional substrate. The following section deals with resonance effects at subwavelength structures caused by localized electron density oscillations. 


\subsubsection{From surface plasmon to nanoantennas}

Surface plasmon are by nature a two dimensional resonance phenomenon at a metal interface. Further restricting the degree of freedom of surface plasmons in metallic wires (one dimensional) or metallic spheres or particles (zero dimensional) gives birth to new resonance phenomena. These phenomena are commonly classified as "antenna effects" and describe interactions of electromagnetic radiation with subwavelength objects at a subwavelength scale. In analogy with antenna theory at radiowave frequencies, there are 2 features these effects are related with: first the enhancement and confinement of propagating optical fields on the nanometer scale ("receiving antenna") and second, the effective release of energy confined on a nanometer scale in propagating optical fields ("transmitting antenna") [56]. It is important to note that for a fluorescent molecule near such an optical antenna, "receiving" and "transmitting" do not occur at the same light frequency. This is because the excitation frequency is always higher than the emission frequency for this molecule. The difference between these frequencies depend on the Stokes shift of the energy states involved in the electronic transition leading to the emission of a fluorescence photon. For this reason, if the antenna efficiently confines and enhances the exciting optical fields it does not mean that fluorescence will be as effectively released in the far-field as the exciting fields are confined and vice versa.

One of the most simple example of nanoantenna is the simple nanorod or singlewire antenna. These are subwavelength structures similar to a cylinder that show a resonant behavior of the light scattering. These structures act in a quite similar fashion to RLC circuits with an external driving force, in this case the electric field of the incoming light wave: the electrons in the structure make forced oscillations. For a light frequency corresponding to the resonance frequency of the equivalent circuit, the amplitude of these oscillations is maximized and a high surface charge density is created at both ends of the nanorod. This surface charge density in turn gives rise to high electric field amplitudes. As a result, the electric fields are not only enhanced at the ends of the nanoantenna compared to the incoming radiation but are also highly confined there.

A bunch of different antenna geometries have been studied so far [56]. The antenna resonances of single triangular structures were also studied [57], [58]. These structures were however triangular metallic structures and not triangular holes in a metallic film as in the case of of the triangular aperture probe. However, comparative studies between crescent-shaped nanoholes in a gold film and gold nanocrescent allows one to envisage that if a gold nanostructure is efficient as a nanoantenna then its complementary form, the same structure as a hole in a gold film is also an efficient antenna. Even if the resonances are not exactly the same in this case, their spectral positions coincide within a few nanometers [59]. This is also what would be expected from the generalized Babinet's principle though it is strictly valid only for perfect conductors. 



\section{Principles of fluorescence correlation spectroscopy}

\subsection{Physics of fluorescence}

\subsubsection{Fundamental aspects}

Fluorescence relates the optical emission characteristic of some particle (molecules or quantum dots) to its light absorption through transition between two electronic states of different energies. In the case of fluorescent molecules, many vibrational states stemming from the nuclei vibrations are superimposed on the possible electronic states. These possible stationary states of a molecule are often illustrated in a so-called Jablonski diagram. Figure 3.1 shows an example of such a diagram.

When a photon impinges on a molecule, there is a certain probability that this photon is absorbed and drives the molecule in an electronic state of higher energy. This absorption probability is given by the absorption cross-section $\sigma_{a b s}$ of the molecule. Following this absorption process, the molecule gives back the energy through a relaxation process. There are several possible relaxation processes for the molecule and fluorescence is one of them. In this case the molecule deexcites in a lower electronic state of the same spin multiplicity, whereas the emitted energy is converted to a photon. But other relaxation processes are also possible: through transition in vibrational-rotational states of lower energy a molecule gives the absorbed energy back in form of heat (Internal conversion, IC). This process is actually always present and explains why the emitted photon mostly has a lower energy than the absorbed photon (Stokes shift).

Additionally to fluorescence and heat transfer other relaxation processes are possible: intersystem crossing is an example. If an electron is excited to a higher electronic state, spin-orbit coupling leads to a finite torque, acting on the spin of that electron. As a result a spin flip can occur, changing the total electronic spin of the molecule. In the two electron case the spin operator $\mathbf{S}$ has two eigenvalues: $s=0$ and $s=1$. The state with value $s=1$ is called the triplet state as the z-component of $\mathbf{S}$ i.e. $S_{z}$ has three possible values $m_{S}=0, \pm 1 / 2$ leading to three states of different energies when placed in a magnetic field. Additionally, the mean distance between two electrons in a triplet state is increased because of exchange interaction between the two spins. As a result, the coulombic repulsion is lowered and the triplet state has a lower energy as the singlet state of same electronic configuration. In figure 3.1 singlet and triplet states are labelled with $S$ 


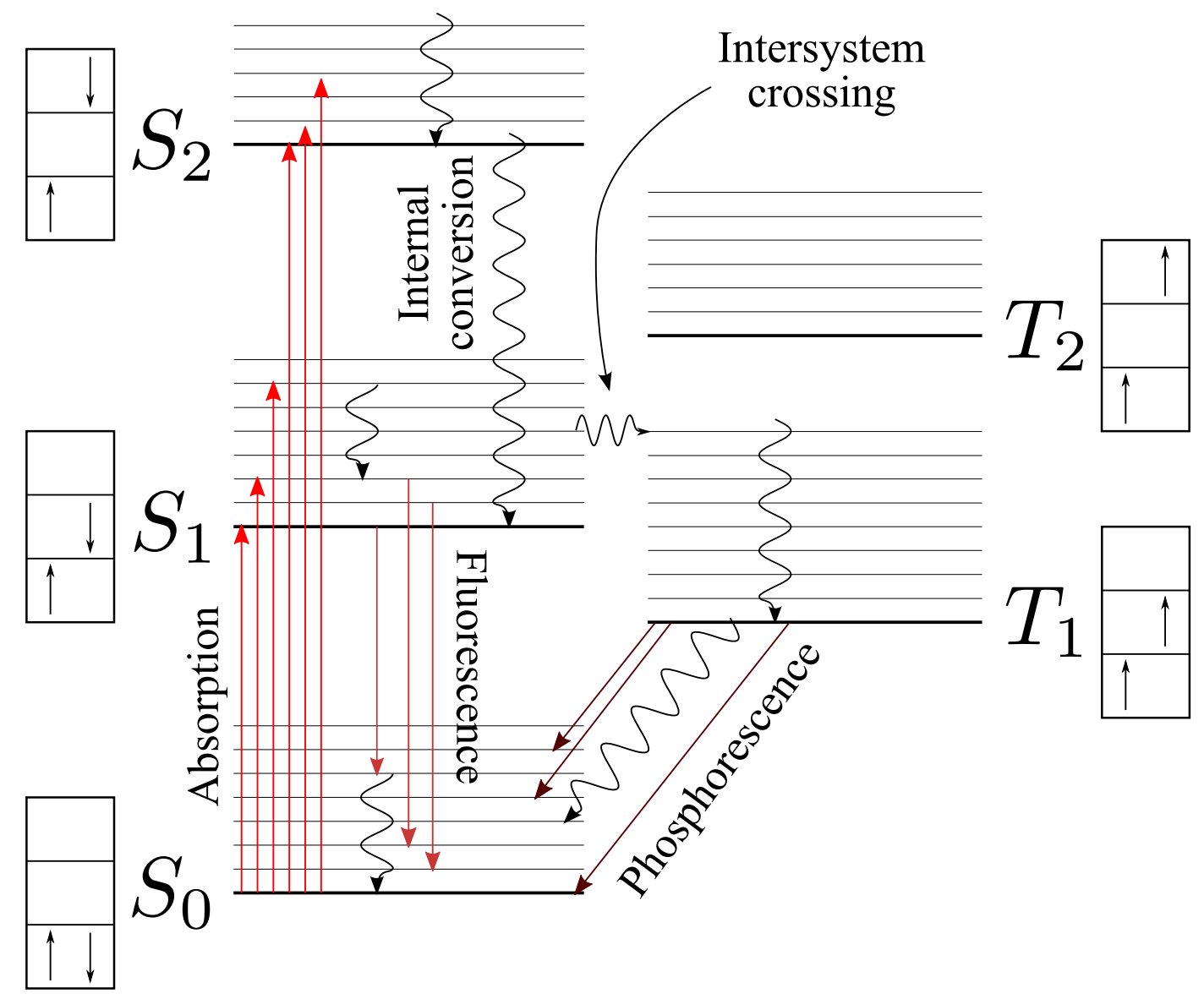

Figure 3.1.: An example of Jablonski diagram for a simplified molecule with two free electrons. The $\mathrm{z}$ orientation of the spin of these electrons is symbolized in the boxes for each electronic configuration. For the 3 electronic configurations and the corresponding wavefunctions on the left, the eigenvalue of the $\mathbf{S}$ operator is $s=0$. These eigenstates are called singlet state. For the two electronic configurations on the right the eigenvalue of $\mathbf{S}$ is $s=1$. These states are called triplet states. Thick lines represent the vibrational-rotational ground state for a given electronic configuration whereas higher vibrational-rotational states are represented with thinner lines. Straight and waved arrows define radiative and nonradiative transitions (internal conversion) respectively. The transition of a singlet to a triplet state is called intersystem crossing. At room temperature, light absorption takes place near the ground vibrational-rotational state. The emission of light through a molecule is termed luminescence and can occur through 2 processes: either the transition occurs between two singlet states and is called fluorescence or it occurs between a triplet state and a singlet state, then it is called phosphorescence. 
and $T$, respectively, with an additional index for a given electronic configuration. This transition between the singlet state and the triplet state, i.e. the intersystem crossing is usually undesired in fluorescence experiment as it hinders the particle to fluoresce. It is one of many types of fluorescence quenching. The efficiency by which a particle can fluoresce is given by its fluorescence quantum yield $Q$, that is defined by the fraction of emitted to absorbed photons. $Q$ is high if, for a molecule in a singlet excited state, transitions to triplet states or non radiative transitions are unlikely to occur.

Transitions from a triplet state to a singlet state usually occurs through relaxation in higher vibrational states of the singlet ground electronic state through heat transfer to the environment. Radiative transition between these two states of different spin multiplicity is a forbidden transition in the dipole approximation framework. As a consequence, this radiative mechanism called phosphorescence is a highly unefficient relaxation process and the triplet state can last as long as a few milliseconds if no other relaxation mechanism is available. Because of this long time, imaging of single molecules leads to "dead time" in the measured fluorescence signal. This phenomenon is called fluorescence intermittency or "blinking", as it repeatedly occurs as the molecule returns from the triplet state to the ground state and is excited again to a higher singlet state where it once again has the possibility to make a transition into the triplet state. Another process that is often linked to the existence of a triplet state in a molecule is the fluorescence bleaching. It refers to the destruction of the molecule through triplet-triplet annihilation with oxygen molecules (ground state is a triplet state). Through interaction with a fluorescence molecule in its triplet state the oxygen molecules can experience a transition to a singlet state that is highly chemically active for this molecule. In this state the oxygen molecule can attack a molecule chemically and may definitively destroy its fluorescence ability.

\subsubsection{The 3-level system}

For a given intensity, i.e. a given constant number of photons impinging on the molecule, a stationary state is reached when each state is populated with a given probability. To better understand this distribution and the consequences on the fluorescence property of the molecule, it is useful to simplify a Jablonski diagram in a three level system where the molecule can populate only 3 different states: the ground singlet state, the first excited singlet state and the triplet ground state. In such a scheme described in figure 3.2, all the possible absorption pathways from the ground state $\left(S_{0}\right)$ to a higher excited state are summed up in one transition to a singlet excited state $\left(S_{1}\right)$. The transition rate of $S_{0}$ to $S_{1}$ is given by $k_{12}$ and scales directly with the excitation intensity as follows:

$$
k_{12}=\frac{\sigma_{\mathrm{abs}} I}{h \nu}
$$


where $\sigma_{\text {abs }}$ is the absorption cross-section of the molecule as introduced above. For liquids, the absorption efficiency is often expressed through the extinction coefficient $\epsilon_{\text {ext }}$. These two quantities are proportional to each other and appear in BeerLambert's law in two different forms: $I=I_{0} \exp \left(-\sigma_{\text {abs }} l N\right)=I_{0} \cdot 10^{-\epsilon_{\text {ext }} l c}$, where $l$ is the distance travelled by the light in the absorbing medium, $N$ the density of absorbing particle and $c$ their concentration in $\mathrm{mol} / \mathrm{L}$. Therefore the absorption cross section $\sigma_{\text {abs }}$ can be expressed as a function of the extinction coefficient through the following relation:

$$
\sigma_{\mathrm{abs}}\left(\mathrm{cm}^{2}\right)=\frac{10^{3} \ln (10)}{N_{a}} \epsilon_{\mathrm{ext}}\left(\mathrm{M}^{-1} \mathrm{~cm}^{-1}\right) \sim 3.8 \cdot 10^{-21} \epsilon_{\mathrm{ext}}\left(\mathrm{M}^{-1} \mathrm{~cm}^{-1}\right)
$$

For example, a fluorescent molecule like Atto655 (Atto-Tec GmbH), has an extinction coefficient of $\epsilon_{\text {ext,Atto655 }}=125000 \mathrm{M}^{-1} \mathrm{~cm}^{-1}$ at the maximum of absorption, i.e. $663 \mathrm{~nm}$ in a phosphate buffer saline $(\mathrm{PBS})$ solvent at $\mathrm{pH}=7.4$ [60]. It corresponds to $\sigma_{\mathrm{abs}, \text { Atto655 }} \sim 4.75 \cdot 10^{-16} \mathrm{~cm}^{2}$.

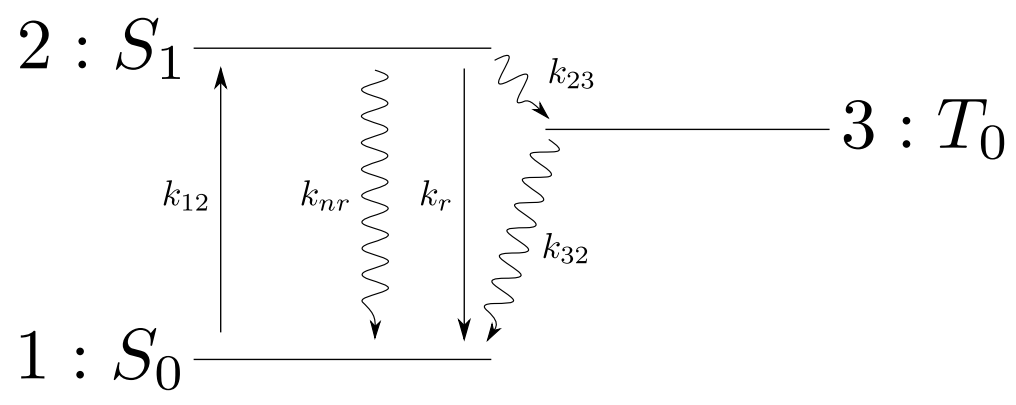

Figure 3.2.: 3-level system of a fluorescent molecule. Only 2 singlet states (the ground state $S_{0}$ and the first excited state $S_{1}$ ) and the ground triplet state are taken into account. All the possible decay pathways are contained in $k_{12}, k_{21}=k_{\mathrm{r}}+k_{\mathrm{nr}}, k_{23}$ and $k_{31}$. Radiative transitions are represented by straight arrows whereas nonradiative transition are represented by wavy arrows.

Furthermore, In the 3-level system, all possible decay pathways from excited singlet states to the ground state are summed up in either a radiative decay rate $k_{\mathrm{r}}$ that corresponds to fluorescence or a nonradiative decay rate $k_{\mathrm{nr}}$ that corresponds to internal conversion. The whole decay rate from the first excited singlet state to the singlet ground state is then: $k_{21}=k_{\mathrm{r}}+k_{\mathrm{nr}}$. Importantly, stimulated emission is not taken into consideration as the emission band of the fluorescent molecules, that is usually used, is located rather far from the excitation wavelength [61].

Finally, this model takes into account the existence of only the triplet ground state. Intersystem-crossing is then allowed only from the first excited singlet state with the rate $k_{23}$. Radiative decay in form of phosphorescence and nonradiative decay can occur between the triplet ground state and the singlet ground state and 
are described by $k_{32}$. Additionally the effect of triplet-triplet annihilation is not taken into account, as it only occurs for high concentration of fluorescent molecules [61].

For a given excitation intensity $I$ the stationary (or steady) state, i.e. the occupation probability of the three different states at equilibrium under the assumption of no coherent excitation is given by:

$$
\begin{aligned}
\overline{x_{1}}+\overline{x_{2}}+\overline{x_{3}} & =1 \\
-k_{12} \overline{x_{1}}+k_{21} \overline{x_{2}}+k_{31} \overline{x_{3}} & =0 \\
k_{23} \overline{x_{2}}-k_{31} \overline{x_{3}} & =0
\end{aligned}
$$

where $\overline{x_{1}}$ is the steady state occupation probability of $S_{0}, \overline{x_{2}}$ the one of $S_{1}$ and $\overline{x_{3}}$ the one of $T_{0}$. The first equation states that the molecule has to be in one of the three states. The two other equations give the rate of change of level $1\left(S_{0}\right)$ and level $3\left(T_{0}\right)$. This rate of change is naturally zero at equilibrium.

Solving this system of equation leads to the following occupation probability for the three different states:

$$
\begin{aligned}
& \overline{x_{1}}=\frac{k_{31}\left(k_{21}+k_{23}\right)}{k_{31}\left(k_{21}+k_{23}\right)+k_{12}\left(k_{31}+k_{23}\right)} \\
& \overline{x_{2}}=\frac{k_{12} k_{31}}{k_{31}\left(k_{21}+k_{23}\right)+k_{12}\left(k_{31}+k_{23}\right)} \\
& \overline{x_{3}}=\frac{k_{12} k_{23}}{k_{31}\left(k_{21}+k_{23}\right)+k_{12}\left(k_{31}+k_{23}\right)}
\end{aligned}
$$

Making use of equation 3.1 and after some transformations, the following more practicable set of equation results:

$$
\begin{aligned}
\overline{x_{1}} & =\frac{1}{1+I / I_{S}} \\
\overline{x_{2}} & =\overline{x_{2, \infty}} \frac{I / I_{S}}{1+I / I_{S}} \\
\overline{x_{3}} & =\overline{x_{3, \infty}} \frac{I / I_{S}}{1+I / I_{S}}
\end{aligned}
$$

The saturation intensity $I_{S}$ at which the singlet ground state occupation has dropped to half of its original value without excitation is given by:

$$
I_{S}=\frac{h \nu}{\sigma} \frac{k_{31}\left(k_{21}+k_{23}\right)}{k_{23}+k_{31}}
$$

and the maximum occupation probabilities $\overline{x_{2, \infty}}$ and $\overline{x_{3, \infty}}$ of the two other states are:

$$
\overline{x_{2, \infty}}=\frac{k_{31}}{k_{23}+k_{31}} \quad \overline{x_{3, \infty}}=\frac{k_{23}}{k_{31}} \overline{x_{2, \infty}}=\frac{k_{23}}{k_{23}+k_{31}}
$$


As an example of numerical values of these different photophysical parameters, Widengren et al. carried out measurements on the fluorescent molecule Rhodamine6G (Rh6G) in some defined environmental conditions, which led to the following values: $k_{21}=250 \cdot 10^{6} 1 / \mathrm{s}[62], k_{23}=1.1 \cdot 10^{6} 1 / \mathrm{s}[61], k_{31}=0.5 \cdot 10^{6} 1 / \mathrm{s}$ [61] and $\sigma_{\text {abs,Rh6G }}=170 \cdot 10^{-18} \mathrm{~cm}^{2}[61]$. At a vacuum wavelength of $\lambda=532 \mathrm{~nm}$ $\left(\nu=5.6 \cdot 10^{14} 1 / \mathrm{s}\right)$, the different occupation probabilities are shown in figure 3.3.

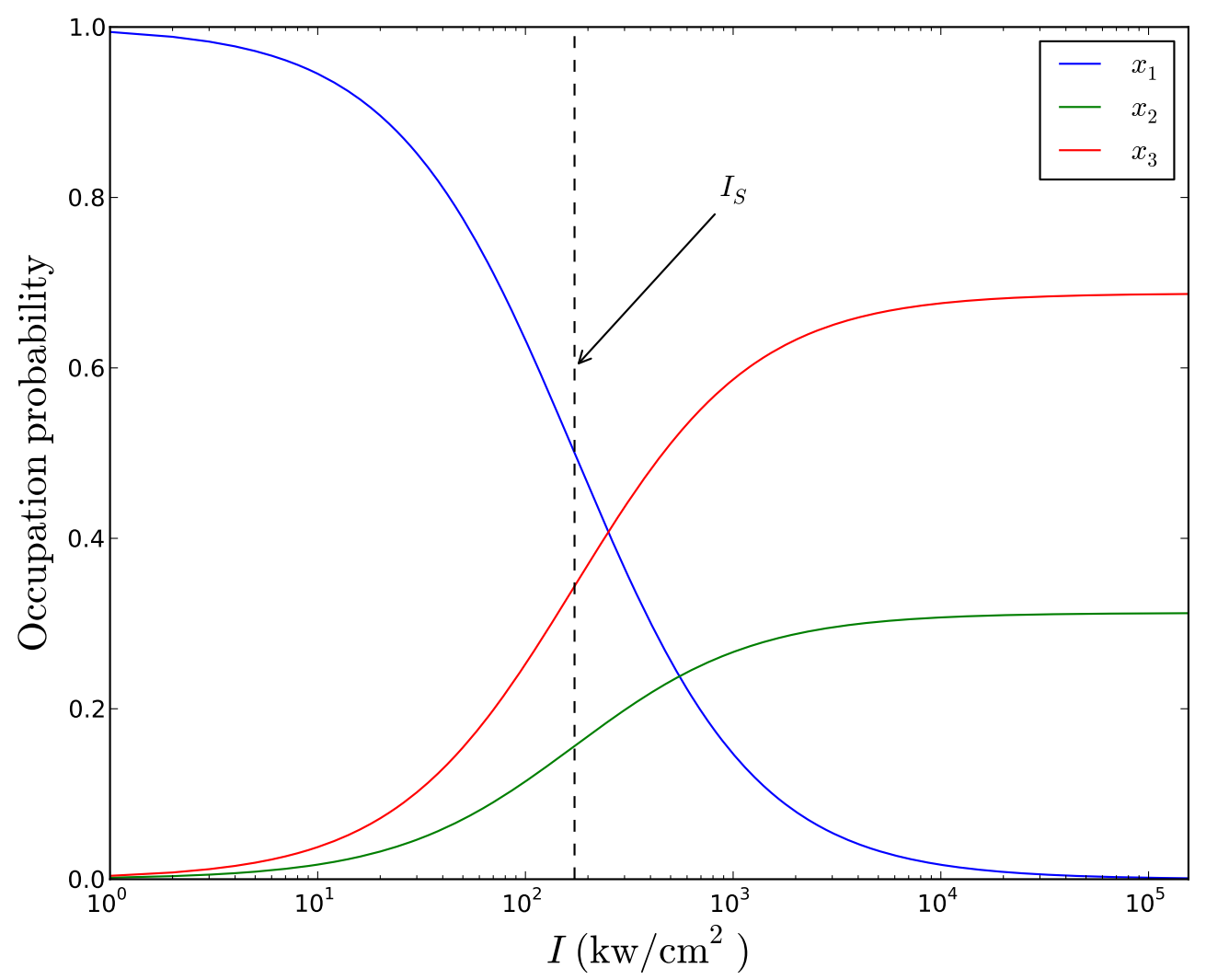

Figure 3.3.: Occupation probabilities of the different states in the 3-level system as a function of the excitation intensity for the fluorescent molecule Rhodamine6G. At small intensities only the ground singlet state is populated. At high intensities it is depopulated and molecules are either in the singlet excited state or in the triplet ground state. For this molecule the saturation intensity is $\sim 172 \mathrm{~kW} / \mathrm{cm}^{2}$.

The rates for Rh6G presented here are of course different for other fluorescent molecules but the qualitative magnitude relationship $k_{21} \gg k_{23}, k_{31}$ is practically always present. The triplet occupation at high excitation intensities can vary depending on the relative magnitude of $k_{23}$ and $k_{31}$. Therefore the following observations from the graph in figure 3.3 are quite general. First, due to the finite and relatively long lifetime of the excited singlet state $\tau_{2}=\frac{1}{k_{23}+k_{21}} \sim \frac{1}{k_{21}}$, the ground singlet state is already strongly depopulated for intensities on the order of $I_{S}$. In 
view of the antenna theory sketched in section 2.2.2, this long lifetime can be seen as a consequence of the mismatch between the electron length confinement (on the order of the molecule size, i.e. a few nanometers) and the emitted wavelength (some hundred nanometers) [56]. This lifetime can be assessed through the knowledge of the fluorescence lifetime $\tau_{f}$ and the quantum yield $Q$ of the molecule. The former is defined by $\tau_{f}=1 / k_{\mathrm{r}}$ and the latter is given by:

$$
Q=\frac{k_{\mathrm{r}}}{k_{\mathrm{r}}+k_{\mathrm{nr}}}=\frac{k_{\mathrm{r}}}{k_{21}}
$$

In the case of Rh6G in water, the quantum yield is $Q=0.95$ [63], meaning that this molecule has very efficient radiative transitions between the two singlet states. Consequently, for the same environmental conditions as for the values of $k_{21}$ and $k_{23}$ that were cited before, the lifetime of the excited singlet state is $\tau_{2}=$ $1 /\left(k_{21}+k_{23}\right) \sim 4$ ns and the fluorescence lifetime is $\tau_{f} \sim \tau_{2} / Q \sim 4.2 \mathrm{~ns}$.

A second aspect shown by the graph is that Rh6G at high intensities has a high steady state triplet state occupation. The relative triplet occupation defined as $\frac{\overline{x_{3}}}{1-\overline{x_{3}}}$ reaches a maximum value of about 2.2 at high intensities. Seen on an ensemble of Rh6G this represents more than $2 / 3$ of the molecules being in their triplet state for high excitation photon rates. This also means that the molecule has an increased chance of being destroyed through the process of triplet-triplet annihilation.

The last aspect that can be seen in the graph concerns the fluorescence emission rate of the molecule. This is defined by:

$$
N_{\text {out }}=Q \cdot k_{21} \cdot \overline{x_{2}}=k_{r} \overline{x_{2}}
$$

However $\overline{x_{2}}$ reaches a maximal value at high intensities. The consequence is that a further increase in excitation intensity will not increase the fluorescence emission: the fluorescence emission saturates. By Rh6G, this maximal value is $N_{\text {out }} \sim 77 \cdot 10^{6} 1 / \mathrm{s}$ but in a real experiment this maximal number of fluorescence photons cannot be detected because of a finite detection solid angle and the optical losses in the setup (see chapter 5).

From this description of the fluorescence properties of a molecule, the effect of fluorescent emission enhancement dealt with in section 2.2 .2 can be understood as the result of a shorter fluorescence lifetime $\tau_{f}$, i.e. a larger radiative decay rate $k_{r}$ that enhances the maximal number $N_{\text {out }}$ of fluorescence photons. However, a competitive phenomenon disturbs this fluorescence enhancement. In order to get to the zone of enhanced intensity, the fluorescent molecules come in close proximity to the metal surface of the nanoantenna. This proximity can cause the molecule to release its energy through absorption in the metal film. In this case, the singlet excited state is quenched and the nonradiative decay $k_{\mathrm{nr}}$ increases [64]. This complex behavior directly influences the quantum yield $Q$ (see equation 3.14) and makes it difficult to understand which part (fluorescence enhancement or fluorescence quenching) is predominant when dealing with nanoantennas. 


\subsection{Fluorescence correlation spectroscopy}

In the precedent section, the level occupation of a fluorescent molecule was studied in a steady state case, i.e. when the equilibrium has already been reached. This is the level occupation that would be measured if, starting with many molecules in their ground states, every molecules were illuminated with a given intensity. After a time long enough counting the number of occurences of one molecule in a given state would give the steady state level occupation of one fluorescent molecule. Alternatively one could operate on a single molecule, letting it go through many excitation cycles and at every given time step, checking in which level it is. The distribution of level occupation would of course be the same.

In a conventional fluorescence experiment, only the steady state occupation probability $\overline{x_{2}}$ is retrieved through the measured fluorescence photons $N_{\text {out }}$. Most parameters characterizing the molecule $\left(k_{21}, k_{31}, \ldots\right)$ cannot be determined in this way. These parameters have an influence on the magnitude and dynamics of the fluctuations in the number of emitted fluorescence photons. As a consequence, analyzing the light emission dynamics as given by the detector should allow one to access these parameters. This is what fluorescence correlation is meant for: a time dependent fluorescence signal $F(t)$ is measured and then autocorrelated. If some events (fluorescence emission, fluorescence dark period) are characterized by a certain time scale, then the correlation of the signal will have a non-zero value over this timescale. If this value is positive the signal is said to be correlated, whereas a negative value corresponds to an anticorrelation. If on average the event shows no correlation for a given time delay, then the value of the correlation function for this time delay is zero.

FCS was first introduced in 1974 to measure chemical kinetic constants and diffusion coefficients in a solution at thermodynamic equilibrium [65]. But it works on the same principle as dynamic light scattering (DLS), using fluorescence instead of scattered light as signal to be correlated. How FCS works is shown in the case of fixed particles and for a diffusion process in a solution in the next two sections.

\subsubsection{Non-diffusing particles}

To better understand how this fluorescence correlation works, it is useful to return to the 3-level system from section 3.1.2 and study the time dependent occupation probabilities $x_{1}(t), x_{2}(t)$ and $x_{3}(t)$. The rate of change $\dot{x}_{1}$ and $\dot{x}_{3}$ are then not zero and they appear correspondingly in the right side of equations 3.4 and 3.5, respectively [29]:

$$
\begin{aligned}
x_{1}(t)+x_{2}(t)+x_{3}(t) & =1 \\
-k_{12} x_{1}(t)+k_{21} x_{2}(t)+k_{31} x_{3}(t) & =\dot{x}_{1}(t) \\
k_{23} x_{2}(t)-k_{31} x_{3}(t) & =\dot{x}_{3}(t)
\end{aligned}
$$


This is a simple linear system of coupled differential equations of first order. Preparing the emitter in the ground state at $t=0\left(x_{1}(t=0)=1\right)$, this sytem is easily solved [29] and gives for the occupation probability of the second level $\left(S_{1}\right)$ :

$$
x_{2}(t)=A_{1} \mathrm{e}^{s_{1} t}+A_{2} \mathrm{e}^{s_{2} t}+A_{3}
$$

In the case where the inequality $k_{21} \geq k_{12} \gg k_{23} \geq k_{31}$ is valid, which is true for most fluorescent molecules, this leads to the following expressions for $A_{1}, A_{2}, A_{3}$, $s_{1}$ and $s_{2}[61]$ :

$$
\begin{aligned}
A_{1} & =-\frac{k_{12}}{k_{12}+k_{21}} \\
A_{2} & =\frac{k_{12}^{2} k_{23}}{\left(k_{12}+k_{21}\right)\left[k_{12}\left(k_{23}+k_{31}\right)+k_{21} k_{31}\right]} \\
A_{3} & =\frac{k_{12} k_{31}}{k_{12}\left(k_{23}+k_{31}\right)+k_{21} k_{31}} \\
s_{1} & =-\left(k_{12}+k_{21}\right) \\
s_{2} & =-\left(k_{31}+\frac{k_{12} k_{23}}{k_{12}+k_{21}}\right)
\end{aligned}
$$

The time dependent occupation probability of level $S_{1}$ is plotted in figure 3.4 using the same parameters for Rh6G from section 3.1.2 for different excitation intensities.

For the curve of $x_{2}(t)$ for the highest intensity, it is clear that the molecule is not likely to be in the excited singlet state for time values smaller than $10^{-11} \mathrm{~s}$. Indeed it is on average still in its ground state since the photon excitation rate is on the order of $1 / k_{12} \sim 4 \cdot 10^{-11} \mathrm{~s}$. Only after a time on this order of magnitude, the molecule will be promoted to the excited state. There, it will dwell as long as no relaxation process takes place. In this excited state and in the 3-level system, there are only two ways to relax: in $S_{0}$ or in $T_{0}$. The first relaxation process occurs after about $1 / k_{21} \sim 4 \cdot 10^{-9} \mathrm{~s}$ and the second after about $1 / k_{23} \sim 10^{-6} \mathrm{~s}$. Therefore, it is highly probable that the molecule stays in the excited state for about $10^{-10}$ to $10^{-9} \mathrm{~s}$. If the molecule relaxes in the ground state $S_{0}$ the high excitation photon rate ensures that the molecule will return to $S_{1}$ in a time $1 / k_{12}$ much smaller than the dwell time $k_{21}$ in $S_{1}$. Therefore, until about $1 / k_{23}$, the molecule is still with high probability in the excited state. However, after this time, a relaxation is possible to the triplet state $T_{0}$. There, it will stay a relatively long time on the order of $1 / k_{31} \sim 2 \mu$ s and can therefore not be excited again to $S_{1}$. As a consequence, the occupation probability of $S_{1}$ decreases until it reaches its steady-state value $x_{2}$ (see equation 3.10) at about $10^{-5} \mathrm{~s}$.

At medium excitation intensities like $100 \mathrm{~kW} / \mathrm{cm}^{2}$, the excitation photon rate is correspondingly smaller and a photon will excite the molecule only after a time of about $1 / k_{12} \sim 20 \mathrm{~ns}$. The deexcitation itself still takes place after $1 / k_{21} \sim 4 \mathrm{~ns}$ and 


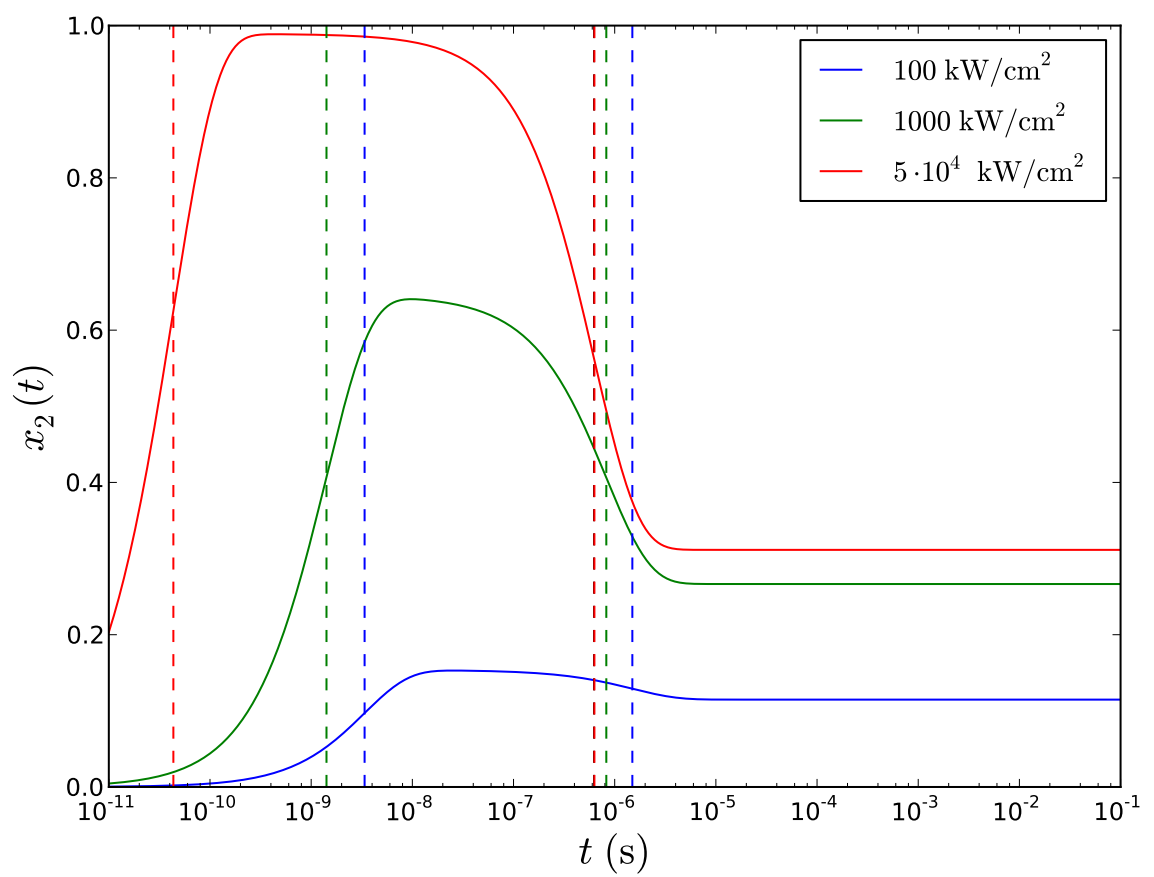

Figure 3.4.: Time dependent occupation probability of the excited singlet state $S_{1}$ for three different excitation intensities. The course of the curve for small lag times is determined by the term $A_{1} \mathrm{e}^{s_{1} t}$ of equation 3.19 and for each curve the position of $-1 / s_{1}$ (the antibunching time) is given by a dashed line. For high excitation intensities, this shifts to smaller lag times and if the resolution capability of the detection instrumentation is not sufficient (see chapter 5), the antibunching behavior cannot be observed accurately. The further course of the curves is determined by $A_{2} \mathrm{e}^{s_{2} t}$ and the corresponding bunching time $-1 / s_{2}$ is given by the dash-dotted line. At higher excitation intensities this bunching time also shifts to smaller lag times but has a limiting value: $-1 /\left(k_{31}+k_{23}\right)$. 
comes much quicker than the excitation. Consequently the molecule is more often in the ground state than in the other states.

The course of $x_{2}(t)$ can be retrieved from the autocorrelation of the fluorescence intensity. This is because the probability for the molecule for emitting a photon is directly proportional to the occupation probability of the excited singlet state. As a result, the mean fluorescence intensity $\langle F(t)\rangle$ at the detector is proportional to the steady state value of $x_{2}(t)$ (equation 3.15). Mathematically, the autocorrelation function $g(\tau)$ is defined as follows:

$$
g(\tau)=\frac{\langle F(t) \cdot F(t+\tau)\rangle}{\langle F(t)\rangle^{2}}
$$

where $\langle\cdots\rangle$ represents time averaging. An alternative definition makes use of the fluctuations of the fluorescence signal $\delta F(t)=F(t)-\langle F(t)\rangle$ :

$$
G(\tau)=\frac{\langle\delta F(t) \cdot \delta F(t+\tau)\rangle}{\langle F(t)\rangle^{2}}
$$

where $g(\tau)$ and $G(\tau)$ are simply related to each other:

$$
g(\tau)=1+G(\tau)
$$

The numerator in equation 3.25 represents the probability of detecting a photon at time $t+\tau$ if a photon was already detected at time $t$ and this is exactly the value given by $x_{2}(\tau)$ in equation 3.19 [29]. The denominator is a normalization factor: it represents the average photon detection rate. Therefore [29]:

$$
g(\tau)=\frac{x_{2}(\tau)}{\overline{x_{2}}}
$$

or alternatively:

$$
G(\tau)=\frac{x_{2}(\tau)}{\overline{x_{2}}}-1
$$

$\tau$ is oftened called lag time. These correlations can be analyzed by the curves in figure 3.4, that have the same course as the curve of $G(\tau)$ as the functions are linearly dependent. For the sake of clarity, the corresponding autocorrelation function $G(\tau)$ is additionally plotted in figure 3.5 for the intensity $1000 \mathrm{~kW} / \mathrm{cm}^{2}$.

At small lag times, the autocorrelation is negative, i.e. the signal is anticorrelated. Following the same arguments as for the explanation of the course of the curve of $x_{2}(t)$, this anticorrelation is due to the fact that, if the molecule has just emitted a photon, it is highly probable that it will not emit any photons in the fraction of time corresponding to $1 /\left(k_{12}+k_{21}\right)$. For longer lag times, a positive correlation appears: depending on the excitation intensity, it becomes more probable to detect a photon at $t+\tau$ if one was already detected at $t$. This is true as long as the molecule has not relaxed to the triplet state, which occurs only at an average time of $\sim 1 / k_{23} \sim 10^{-6}$ s after an excitation in $S_{1}$. For longer lag times the correlations 


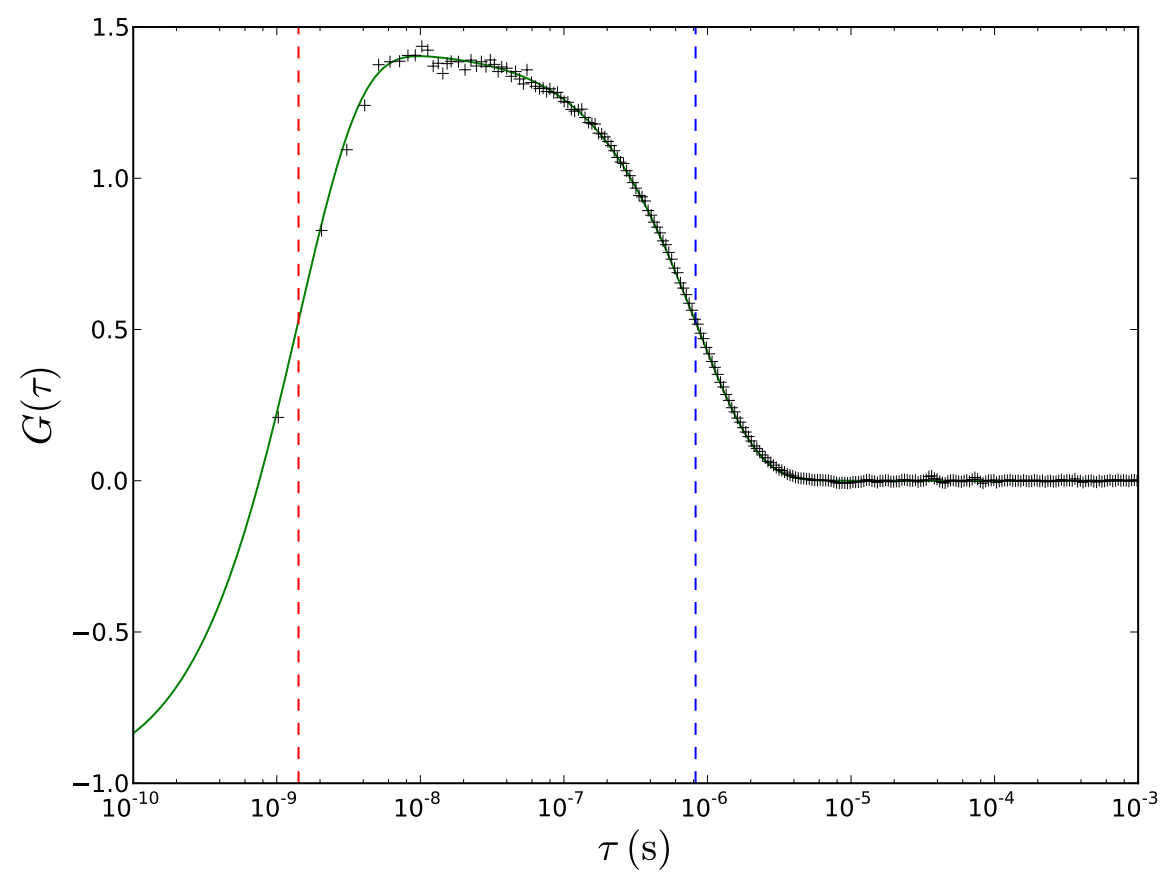

Figure 3.5.: Autocorrelation calculated with equation 3.29 (green curve). $G(\tau)$ is given by $x_{2}(\tau)$ in equation 3.19 . The time rates $-1 / s_{1}$ resp. $-1 / s_{2}$ are also given in red and blue, respectively. The black points represent the computed autocorrelation from the simulation of a molecule with the parameters of Rh6G at the same excitation intensity of $1000 \mathrm{~kW} / \mathrm{cm}^{2}$ used for the green curve. Simulation data are generated with the model presented in section 4.1 and the calculation of the autocorrelation is carried out by a multiple-tau algorithm (see chapter 5 ). 
disappear: at such long lag times, the molecule has undergone many excitations so that it is unclear in which state the molecule is. Therefore, it cannot be said whether a photon will be detected or not.

Although the time dependent fluorescence signal $F(t)$ at first glance shows a somehow random nature, the corresponding correlation times, i.e. the time ranges over which correlations (positive or negative) can be observed, are yet visible. This signal is called the time trace and such a time trace was simulated in figure 3.6. The details of the simulation are explained in section 4.1. Briefly, the simulation was carried out by using the fact that the probability for the molecule to make a transition to another level in a given time follows a Poisson distribution, which is equivalent to draw the time between two transitions from an exponential distribution where the rate parameter $\lambda$ is chosen according to the transition $\left(1 / k_{12}\right.$ for the transition $S_{0} \rightarrow S_{1}, 1 / k_{23}$ for $S_{1} \rightarrow T_{0}$ and so on). The autocorrelation of this time trace gives the point of the black curve of figure 3.5, properly fitting to the analytical solution from equation 3.29.

This time trace over the first $35 \mu$ s of the simulation is actually only a small section of its entire simulation duration $(100 \mathrm{~ms})$. At the intensity of $1000 \mathrm{~kW} / \mathrm{cm}^{2}$, a lot of photon detection events can already be seen, although only one fluorescent photon from ten was supposed to be detected. The simulation gave a total count rate of $6.7 \cdot 10^{3} \mathrm{kHz}$, in accordance with the steady-state emission count rate calculated from equations 3.15 and 3.10 given the emission probability detection of 0.1 of the simulation and a simplified singlet excited to singlet ground state transition rate of $k_{\mathrm{r}}=k_{21}=250 \cdot 10^{6} 1 / \mathrm{s}$. This steady-state emission count rate is $\sim 76 \cdot 10^{3} \mathrm{kHz}$.

In this time trace the two different correlation times are well visible. Mostly the signal appears in "bursts" of photons which are only interrupted when the molecule dwells in the triplet state, where no photon emission is possible (blue arrow of figure 3.6). This "triplet time" also called "bunching time" is on the order of a microsecond with fluctuations according to the exponential distribution of the time between two triplet events. On a smaller time scale of one microsecond (see the inset of figure 3.6, that corresponds to the enlargement of the first burst of photons occuring in the first microsecond), the time between the emission of two photons is depicted with red arrows. This is called "antibunching time" because in this period of time no photon can be emitted due to the fact that the particle is still in the singlet ground state. This strongly contrasts with the bunching time, a period of time in which photons are detected in close temporal proximity. During the photon bursts, the molecule experiences many excitation-deexcitation cycles between $S_{0}$ and $S_{1}$ and will accordingly emit a photon in average every $1 / k_{12}+1 / k_{21}$ seconds.

To finish this introductory section on fluorescence correlation technique on fixed fluorescent molecules, a word on terminology: in this work, no spectroscopic measurements were carried out in combination with this correlation technique. It is yet always called fluorescence correlation spectroscopy (FCS). Therefore, we will stick to the abbreviation FCS throughout the rest of this text. 


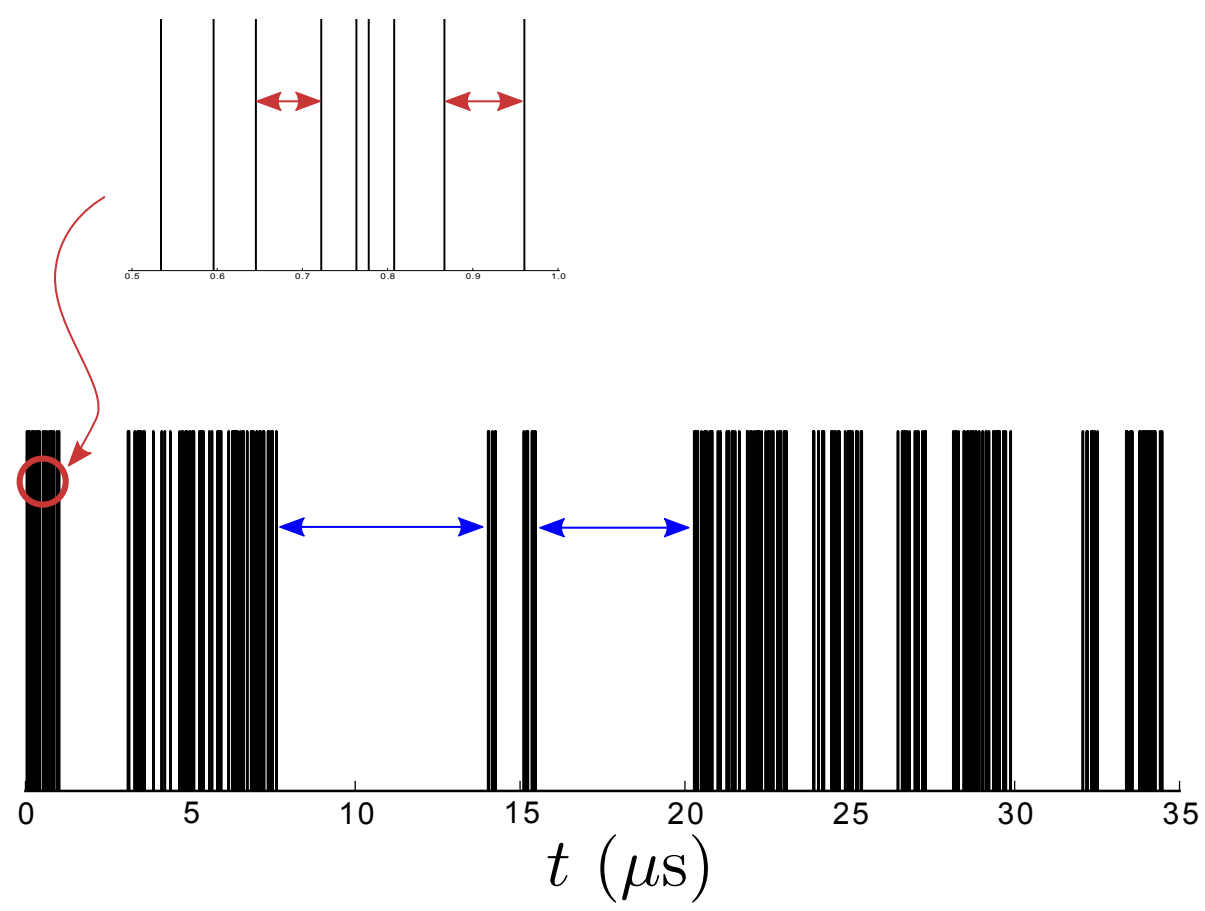

Figure 3.6.: An example of the time representation of $F(t)$, also called time trace. This is the result of the simulation of a molecule being excited at $I=$ $1000 \mathrm{~kW} / \mathrm{cm}^{2}$ and for which the detection probability of an emitted photon is 0.1 . This time trace is only a small part $(35 \mu \mathrm{s})$ of the total simulation duration of $100 \mathrm{~ms}$. As the processes leading to the photon detection events are of stochastic nature, this time trace is only of illustrative nature and differs at a different time or in another simulation. The photon detection events are represented with bars of $1 \mathrm{~ns}$ width. The inset is an enlargement of the first microsecond of the time trace. The blue arrows represent the time between the emission of two photons when the molecule relaxed in the triplet state in between ("triplet time" or "bunching time"). The red arrows, on the other hand, represent the time between the emission of two photon when the molecule directly relaxed in the singlet ground state between the two photon emission events ("antibunching time"). Because of the stochastic nature of the transition times between different energy levels, ascribing the arrows to the bunching or antibunching time is not unambiguous. Only the long time delay in case of the blue arrows and the small time delay in case of the red arrows, respectively, enables to state that these arrows represent bunching and antibunching time, respectively, with high probability. 


\subsubsection{Particles diffusing through a focussed Gaussian beam}

The focused Gaussian beam is an idealisation of the field intensity distribution in a focused laser beam. Indeed a Gaussian excitation profile means a Gaussian spectrum of spatial frequencies with consequently an infinite extension of the spectrum into high spatial frequencies. However, as seen in section 2.1.1 not all the spatial frequencies, especially those of high amplitudes can propagate in the far-field. Therefore, a Gaussian spectrum and a Gaussian excitation profile cannot exist. It is yet a good approximation of the distribution of intensity in a focused laser beam and is used for analytical purposes.

The first implementation of FCS was done using a focused laser beam to measure, among other things, diffusion coefficients in a solution at thermodynamical equilibrium [65]. It is based on the high fluorescence fluctuations stemming from the diffusion of a fluorescent molecule in the spatially inhomogeneous Gaussian beam (see figure 3.7). The idea is that if the molecule emitted a photon at an instant $t$, the probability that it emits another photon at time $t+\tau$ is now not only dependent on the different transition rates of the molecule (see section 3.2.1) but also on the location within the Gaussian beam. Even if the movements of a molecule in a solution at thermodynamical equilibrium are totally random, its position in the solution is governed by a probability distribution, which is reflected by the diffusion law (Fick's second law):

$$
\frac{\partial C(\vec{r}, t)}{\partial \tau}=D \Delta C(\vec{r}, t)
$$

where $C(\vec{r}, t)$ is the molecule's concentration in the solution, dependent on time $t$, the position of the molecule $\vec{r}$ and the fluctuations about the mean concentration $C_{0}: \delta C(\vec{r}, t)=C(\vec{r}, t)-C_{0} . D$ is called the diffusion coefficient and is given in the simplest model of spheres by the Stokes-Einstein equation:

$$
D=\frac{k_{B} T}{6 \pi \eta R_{0}}
$$

with the Boltzmann constant: $k_{B}=1.381 \cdot 10^{-23} \mathrm{JK}^{-1}$, the dynamic viscosity $\eta$ of the solution $\left(\eta=1.002 \cdot 10^{-3}\right.$ Pa.s for water at $\left.20^{\circ} \mathrm{C}\right)$ and the radius $R_{0}$ of the sphere. When used to compute the effective size of a particle that is generally not spherical from its diffusion coefficient, $R_{0}$ is called the hydrodynamic radius of the particle.

To understand the correlation that arises from the diffusion of the molecules in and out of the volume illuminated by a Gaussian beam, it is useful to first neglect the correlation that stems from bunching (transition in the triplet state) and antibunching (finite excitation and deexcitation time of the singlet states). In this case the fluorescence intensity measured can be expressed as [66]:

$$
F(t)=K \int_{-\infty}^{+\infty} \operatorname{CEF}(\mathbf{r}) I_{\operatorname{exc}}(\mathbf{r}) C(\mathbf{r}, t) \mathrm{d} V
$$




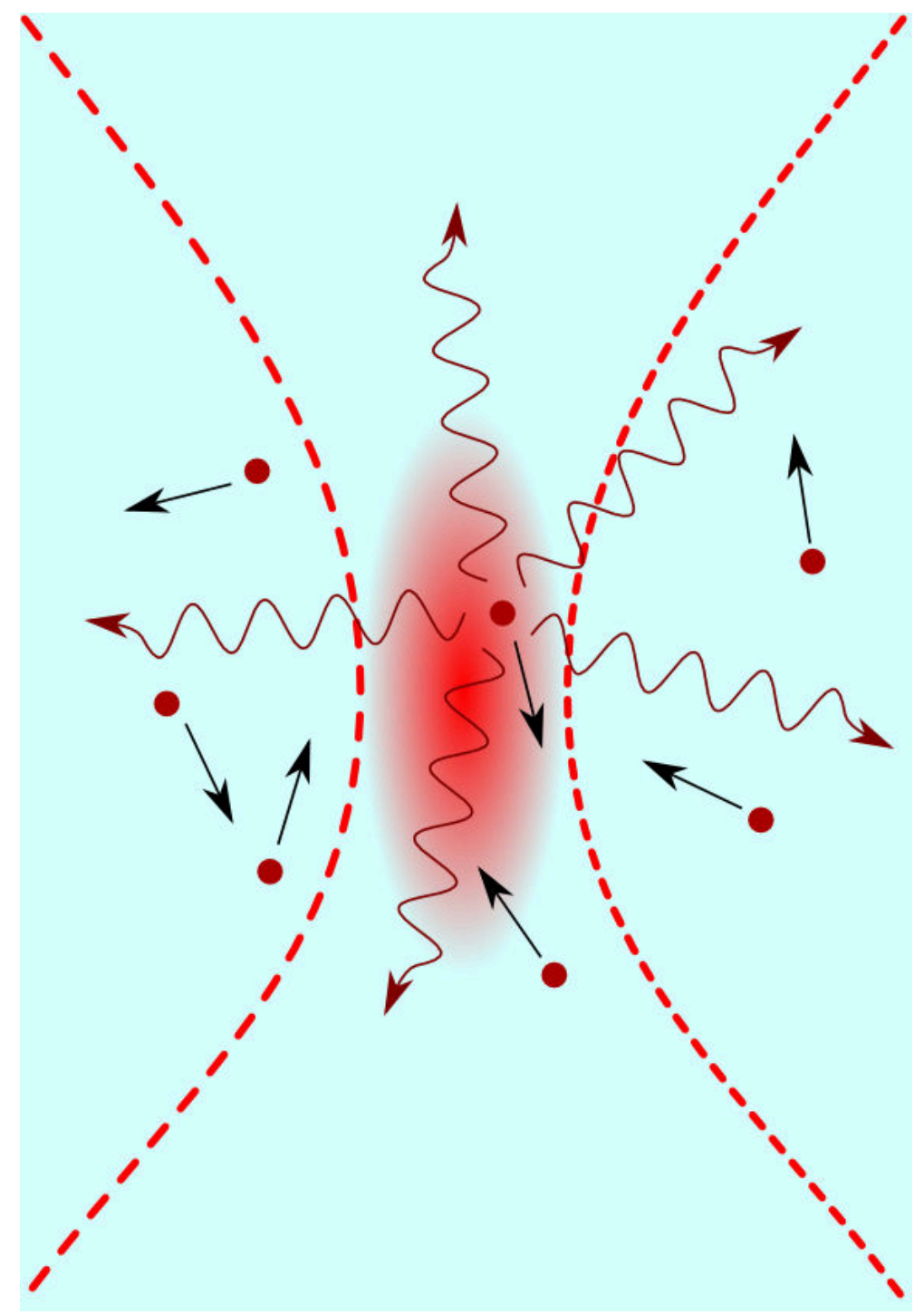

Figure 3.7.: Diffusion of fluorescent molecules represented here as small spheres in a Gaussian beam. The black straight arrows refer to the random movement of the molecules in the solution. If a molecule enters the Gaussian beam it emits fluorescence isotropically, which is represented by waved arrows. The red dashed line shows the intensity profile of the laser beam. 
where $\operatorname{CEF}(\mathbf{r})$ is the collection efficiency function, which includes the fluorescence quantum efficiency and $K$ is a constant factor depending on the absorption crosssection of the molecule and its quantum yield. Usually $\operatorname{CEF}(\mathbf{r})$ varies little on the scale of $I_{\text {exc }}(\mathbf{r})$ and can be expressed through a collection efficiency factor $C$. Consequently the fluorescence signal can be written:

$$
F(t)=K^{\prime} \int_{-\infty}^{+\infty} p(\mathbf{r}) C(\mathbf{r}, t) \mathrm{d} V
$$

with $p(\mathbf{r})$ being the Gaussian excitation profile normalized by the excitation intensity $I_{0}$ at maximum:

$$
p(\mathbf{r})=\frac{I_{\text {exc }}}{I_{0}}=\mathrm{e}^{-2 \frac{x^{2}+y^{2}}{R_{x y}^{2}}} \mathrm{e}^{-2 \frac{z^{2}}{R_{z}^{2}}}
$$

where $K^{\prime}=K \cdot C$ and $R_{x y}$ and $R_{z}$ refer to the distance at which the intensity falls off to $I_{0} / \mathrm{e}^{2}$.

Using the definition of the autocorrelation function from equation 3.26, it follows:

$$
G(\tau)=\frac{K^{\prime 2} \iint_{-\infty}^{+\infty} p(\mathbf{r}) p\left(\mathbf{r}^{\prime}\right)\langle\delta C(\mathbf{r}, t) \delta C(\mathbf{r}, t+\tau)\rangle \mathrm{d} V \mathrm{~d} V^{\prime}}{K^{\prime 2}\left(\int_{-\infty}^{+\infty} p(\mathbf{r}\langle C(\mathbf{r}, t)\rangle \mathrm{d} V)^{2}\right.}
$$

The process of diffusion in the solution at thermodynamical equilibrium can be considered as a stationary process, therefore $G(\tau)$ further simplifies to:

$$
G(\tau)=\frac{\iint_{-\infty}^{+\infty} p(\mathbf{r}) p\left(\mathbf{r}^{\prime}\right)\left\langle\delta C(\mathbf{r}, 0) \delta C\left(\mathbf{r}^{\prime}, \tau\right)\right\rangle \mathrm{d} V \mathrm{~d} V^{\prime}}{C_{0}^{2}\left(\int_{-\infty}^{+\infty} p(\mathbf{r}) \mathrm{d} V\right)^{2}}
$$

where $\int_{-\infty}^{+\infty} p\left(\mathbf{r} \mathrm{d} V=V_{\text {exc }}\right.$ is the excitation volume defined by the Gaussian beam where the excitation intensity is larger than $I_{0} / \mathrm{e}^{2}$.

Furthermore, the concentration fluctuations $\delta C(\mathbf{r}, t)$ can be retrieved through the diffusion equation 3.30 by applying a Fourier transformation on the component $\mathbf{r}$ of the concentration fluctuations $\delta C(\mathbf{r}, t)[66]$ :

$$
\begin{aligned}
\frac{\partial C(\mathbf{r}, t)}{\partial t} & =D \nabla^{2} C(\mathbf{r}, t) \\
\Rightarrow \frac{\partial \delta C(\mathbf{r}, t)}{\partial t} & =D \nabla^{2} \delta C(\mathbf{r}, t) \\
\Rightarrow \mathcal{F}_{\mathbf{r} \rightarrow \mathbf{k}}\left\{\frac{\partial \delta C(\mathbf{r}, t)}{\partial t}\right\} & =\mathcal{F}_{\mathbf{r} \rightarrow \mathbf{k}}\left\{D \nabla^{2} \delta C(\mathbf{r}, t)\right\} \\
\Rightarrow \frac{\partial \delta \tilde{C}(\mathbf{k}, t)}{\partial t} & =-D \mathbf{k}^{2} \delta \tilde{C}(\mathbf{k}, t)
\end{aligned}
$$


where $\delta \tilde{C}(\mathbf{k}, t)$ is the Fourier transform of $\delta C(\mathbf{r}, t)$. The last differential equation has the solution:

$$
\delta \tilde{C}(\mathbf{k}, t)=\delta \tilde{C}(\mathbf{k}, 0) \mathrm{e}^{-D \mathbf{k}^{2} t}
$$

This can be used to calculate the term in bracket in equation 3.36:

$$
\begin{aligned}
\left\langle\delta C(\mathbf{r}, 0) \delta C\left(\mathbf{r}^{\prime}, \tau\right)\right\rangle & =\left\langle\delta C(\mathbf{r}, 0) \mathcal{F}_{\mathbf{k}^{\prime} \rightarrow \mathbf{r}^{\prime}}^{-1}\left\{\delta \tilde{C}\left(\mathbf{k}^{\prime}, \tau\right)\right\}\right\rangle \\
& =\mathcal{F}_{\mathbf{k}^{\prime} \rightarrow \mathbf{r}^{\prime}}^{-1}\left\{\left\langle\delta C(\mathbf{r}, 0) \delta \tilde{C}\left(\mathbf{k}^{\prime}, \tau\right)\right\rangle\right\} \\
& =\mathcal{F}_{\mathbf{k}^{\prime} \rightarrow \mathbf{r}^{\prime}}^{-1}\left\{\left\langle\delta C(\mathbf{r}, 0) \delta \tilde{C}\left(\mathbf{k}^{\prime}, 0\right) \mathrm{e}^{-D \mathbf{k}^{\prime 2} \tau}\right\rangle\right\} \\
& =\mathcal{F}_{\mathbf{k}^{\prime} \rightarrow \mathbf{r}^{\prime}}^{-1}\left\{\left\langle\delta C(\mathbf{r}, 0) \mathcal{F}_{\mathbf{r}^{\prime} \rightarrow \mathbf{k}^{\prime}}\left\{\delta C\left(\mathbf{r}^{\prime}, 0\right)\right\} \mathrm{e}^{-D \mathbf{k}^{\prime 2} \tau}\right\rangle\right\} \\
& =\mathcal{F}_{\mathbf{k}^{\prime} \rightarrow \mathbf{r}^{\prime}}^{-1}\left\{\mathrm{e}^{-D \mathbf{k}^{\prime 2} \tau} \mathcal{F}_{\mathbf{r}^{\prime} \rightarrow \mathbf{k}^{\prime}}\left\{\left\langle\delta C(\mathbf{r}, 0) \delta C\left(\mathbf{r}^{\prime}, 0\right)\right\rangle\right\}\right\}
\end{aligned}
$$

Since the number of molecules within any subvolume is supposed to be Poisson distributed (mean number of molecules is equal to its variance) and the molecules do not interact with each other [66]:

$$
\begin{aligned}
\left\langle\delta C(\mathbf{r}, 0) \delta C\left(\mathbf{r}^{\prime}, 0\right)\right\rangle & =\left\langle\left(C(\mathbf{r}, 0)-C_{0}\right)\left(C\left(\mathbf{r}^{\prime}, 0\right)-C_{0}\right)\right\rangle \\
& =\left\langle C(\mathbf{r}, 0)^{2}\right\rangle \delta\left(\mathbf{r}-\mathbf{r}^{\prime}\right)-C_{0}^{2} \\
& =C_{0} \delta\left(\mathbf{r}-\mathbf{r}^{\prime}\right)
\end{aligned}
$$

Further using the properties of the Fourier transform, the numerator of equation 3.36 becomes:

$$
\iint_{-\infty}^{+\infty} \tilde{p}(\mathbf{k}) \tilde{p}\left(\mathbf{k}^{\prime}\right) \mathcal{F}_{\mathbf{r} \rightarrow \mathbf{k}}\left\{\left\langle\delta C(\mathbf{r}, 0) \delta C\left(\mathbf{r}^{\prime}, \tau\right)\right\rangle\right\} \mathrm{d} \mathbf{k} \mathrm{d} \mathbf{k}^{\prime}
$$

which under use of equations 3.46 and 3.49 simplifies to:

$$
\int_{-\infty}^{+\infty} \tilde{p}(\mathbf{k})^{2} C_{0} \mathrm{e}^{-D \mathbf{k}^{2} \tau} \mathrm{d} \mathbf{k}
$$

with $\tilde{p}(\mathbf{k})$ being the Fourier transform of the normalized excitation profile of equation 3.34:

$$
\tilde{p}(\mathbf{k})=\frac{1}{8} R_{x y}^{2} R_{z} \mathrm{e}^{-\frac{\left(k_{x}^{2}+k_{y}^{2}\right) R_{x y}^{2}}{8}} \mathrm{e}^{-\frac{k_{z}^{2} R_{z}^{2}}{8}}
$$

Upon integration of the numerator and the denominator, equation 3.36 finally reads:

$$
G(\tau)=\frac{1}{C_{0} V_{\text {exc }}} \frac{1}{1+\frac{4 D \tau}{R_{x y}^{2}}} \frac{1}{\sqrt{1+\frac{4 D \tau}{R_{z}^{2}}}}
$$


where $V_{\text {exc }}$ has the dimension of a volume and is given by:

$$
V_{\mathrm{exc}}=\pi^{3 / 2} R_{x y}^{2} R_{z}
$$

As the Gaussian beam has a continuous intensity profile, a volume cannot strictly be defined as some volume element delimited by a sharp border but this volume is slightly larger than the volume $V_{\text {eps }}$ of a prolate spheroide described by an equatorial radius of $R_{x y}$ and a polar radius $R_{z}$. At these radii, the intensity falls off by a factor of $1 / \mathrm{e}^{2}$ of the value in the center of the beam: $V_{\text {exc }} \sim 1.3 V_{\text {eps }}$.

The curve resulting from equation 3.53 is shown in figure 3.8 where the factor $C_{0} V_{\text {exc }}$ defines a number $N$ of molecules present in average in the excitation volume $V_{\text {exc }}$. As this number increases, the amplitude of $G(\tau)$ decreases. This corresponds to the intuitive idea that fluctuations of intensity are all the more observable as the number of molecules participating to this fluctuations by diffusing in and out of the excitation volume is small.

Note that the value $G(0)=1 / N$ of the autocorrelation function at zero lag time is independent of the precise shape of the volume. It is also valid for all diffusion processes where possible additional fluctuation sources (of photophysical nature like bunching or antibunching or of chemical nature like creation or destruction processes of the molecules) are not taken into account. This value can therefore be retrieved without giving explicitely $I_{\text {exc }}(\mathbf{r})$ or $\mathrm{CEF}(\mathbf{r})$ but just using Poisson statistics and the fact that the fluorescence signal is proportional to the number of fluorescent molecules that contribute to the signal: $F(t)=\alpha N(t)$. As the number of molecules in any subvolume of the solution is Poisson distributed, its variance $\sigma_{N}^{2}=\left\langle\delta N(t)^{2}\right\rangle$ is the same as its mean: $\left\langle\delta N(t)^{2}\right\rangle=\left\langle N(t)^{2}\right\rangle-\langle N(t)\rangle^{2}=\langle N(t)\rangle$ and consequently:

$$
G(0)=\frac{\left\langle\delta F(t)^{2}\right\rangle}{\langle F(t)\rangle^{2}}=\frac{\left\langle\delta N(t)^{2}\right\rangle}{\langle N(t)\rangle^{2}}=\frac{1}{\langle N(t)\rangle}
$$

With the knowledge of the concentration of fluorescent molecules in the solution, the extent of the volume out of which the molecules contribute to the fluorescence signal can be retrieved, provided that no other fluctuation source is present.

Two correlation times can be defined from equation 3.53: the lag times $\tau$ at which the term $\left(1+R_{i}^{2} \tau / 4 D\right)^{-1 / 2}$ falls to $1 / \sqrt{2}$ with $i=\{x, y, z\}$. In the case of a Gaussian beam with axis symmetry about $z, R_{x}=R_{y}=R_{x y}$ and the correlation time relating to the diffusion in the $(x, y)$-plane is $\tau_{x y}=R_{x y}^{2} / 4 D$. For the diffusion in the $z$ direction, $\tau_{z}=R_{z}^{2} / 4 D$.

These correlation times have a form similar to the time obtained from the consideration of the variance of the molecule displacement in solution: For a $n$-dimensional displacement the variance of the random variable $\vec{X}=\left(X_{1}, X_{2}, \ldots, X_{n}\right)$ is given by:

$$
\sigma_{\vec{X}}^{2}=\left\langle\vec{X}^{2}\right\rangle-\langle\vec{X}\rangle^{2}=2 n D t \quad \Rightarrow \quad \tau=\frac{\sigma_{\vec{X}}^{2}}{2 n D}
$$




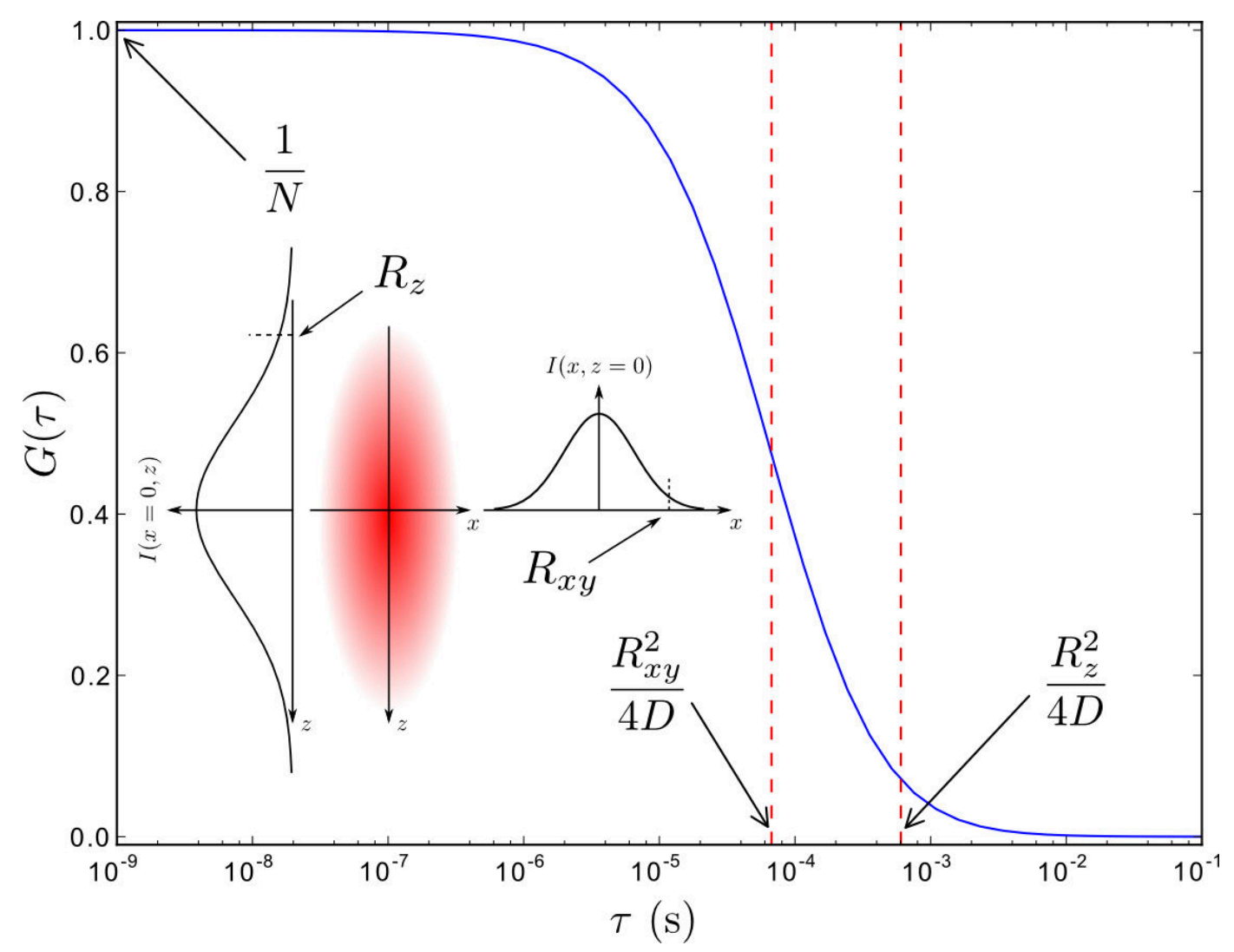

Figure 3.8.: Autocorrelation of the fluorescence intensity of molecules in a solution diffusing in and out a Gaussian beam, which excitation profile is given by 3.53. The excitation profile is also sketched in the inset with $R_{x y}$ and $R_{z}$ being the radii at which the intensities $I(x, z=0)$ and $I(x=0, z)$, respectively, are a factor $1 / \mathrm{e}^{2}$ smaller than the maximum intensity in the center of the beam. The corresponding time scale $R_{x y}^{2} / 4 D$ and $R_{z}^{2} / 4 D$ for the diffusion process are indicated on the graph. The maximum of the autocorrelation occurs at small lag times $\tau \rightarrow 0$ with $N=C_{0} V$ being the mean number of molecules in a volume $V=\pi^{3 / 2} R_{x y}^{2} R_{z}$. 
Letting $\sigma_{\vec{X}}^{2}$ be the variance of the displacement in the two dimensional plane $(x, y)$ then $\tau=\sigma_{(X, Y)}^{2} / 4 D$. The similarity of this expression with $\tau_{x y}$ shows that the correlation time over which the fluorescence emission takes place is a measure of the mean time needed to cross the excitation volume in the $(x, y)$-plane.

In figure 3.8, $\tau_{x y}$ and $\tau_{z}$ are depicted by the dashed line. At these positions, a transition between positive correlations (positive values of the $G(\tau)$ ) and no correlation (zero value of $G(\tau)$ ) is observed. The fact that at small lag times, only a positive correlation is observed stems from the fact that antibunching was not considered in the derivation of the autocorrelation. Consequently, if a molecule emitted a photon at $t=0$ it means it is in the excitation volume and only little time afterwards the molecule is likely to be still in this excitation volume and it is consequently likely to emit another photon.

Measuring the autocorrelation of the fluorescence of a molecule arising from the diffusion in and out of a focused laser beam with a molecule of known diffusion coefficient $D$, is a widespread technique as it allows to assess the dimensions $R_{x y}$ and $R_{z}$ of the focal spot of the laser by fitting the autocorrelation to equation 3.53. Inversely, it can also be used to determine a diffusion coefficient with the precise knowledge of $R_{x y}$ ans $R_{z}$. However, some artifacts can impair this measurement. One of them is the triplet excitation of the diffusing molecules. As shown in section 3.2.1 transient relaxation in the triplet state also leads to fluctuations of the fluorescence intensity. Widengren et al. showed that these additional fluctuations during a diffusional process can be taken into account with an additional multiplying factor compared to equation 3.53, which contains the whole triplet dynamics. The new autocorrelation function accouting for the triplet dynamics is, however, only valid for a mean triplet time much smaller than the mean diffusion time, so that the triplet occupation of the diffusing molecules can be considered to be in a steady state during the diffusion process [62]:

$$
G(\tau)=\frac{1}{N} \frac{1}{1+\frac{4 D \tau}{R_{x y}^{2}}} \frac{1}{\sqrt{1+\frac{4 D \tau}{R_{z}^{2}}}}\left(1+\frac{T}{1-T} \mathrm{e}^{-\frac{\tau}{\tau_{T}}}\right)
$$

where $T$ is the steady state occupation probability of the triplet state $\overline{x_{3}}$ as given by the equation 3.8 and $\tau_{T}$ is the bunching time given by $-1 / s_{2}$ in equation 3.19 . For both $T$ and $\tau_{T}$ the rate constant $k_{12}$ is approximated for a constant intensity profile $I_{T}$ in the transverse plane of the laser beam, so that the corresponding power $I_{T} \pi R_{x y}^{2}$ is the same as the power of the Gaussian beam:

$$
I_{T} \pi R_{x y}^{2}=\int_{-\infty}^{+\infty} I_{0} \mathrm{e}^{-2 \frac{x^{2}+y^{2}}{R_{x y}^{2}}} \mathrm{~d} V=\frac{I_{0}}{2} \pi R_{x y}^{2} \quad \Rightarrow \quad I_{T}=\frac{I_{0}}{2}
$$

A more detailed description of the autocorrelation function for triplet occupation probability $T$ depending on the distribution of the intensity in the Gaussian beam, is given in [61]. A typical autocorrelation accounting for triplet is sketched in figure 3.9 for $T=0.3$ and $\tau_{T}=1 \mu \mathrm{s}$. The main feature is that the value of the autocorrelation function at lag time $\tau=0$ is no longer the inverse of the number of 
molecules $N$ in the excitation volume but the inverse of the number of molecules less the ones in the triplet state: $N(1-T)$. Additionally, the intensity fluctuations owing to the transition in the triplet state are clearly taking place on a time scale much lower than the mean diffusion time in and out of the excitation volume. This observation allows the assumption of the triplet occupation of the molecule to be in its steady-state value during the diffusion process. This assumption was used to derive equation 3.57 .

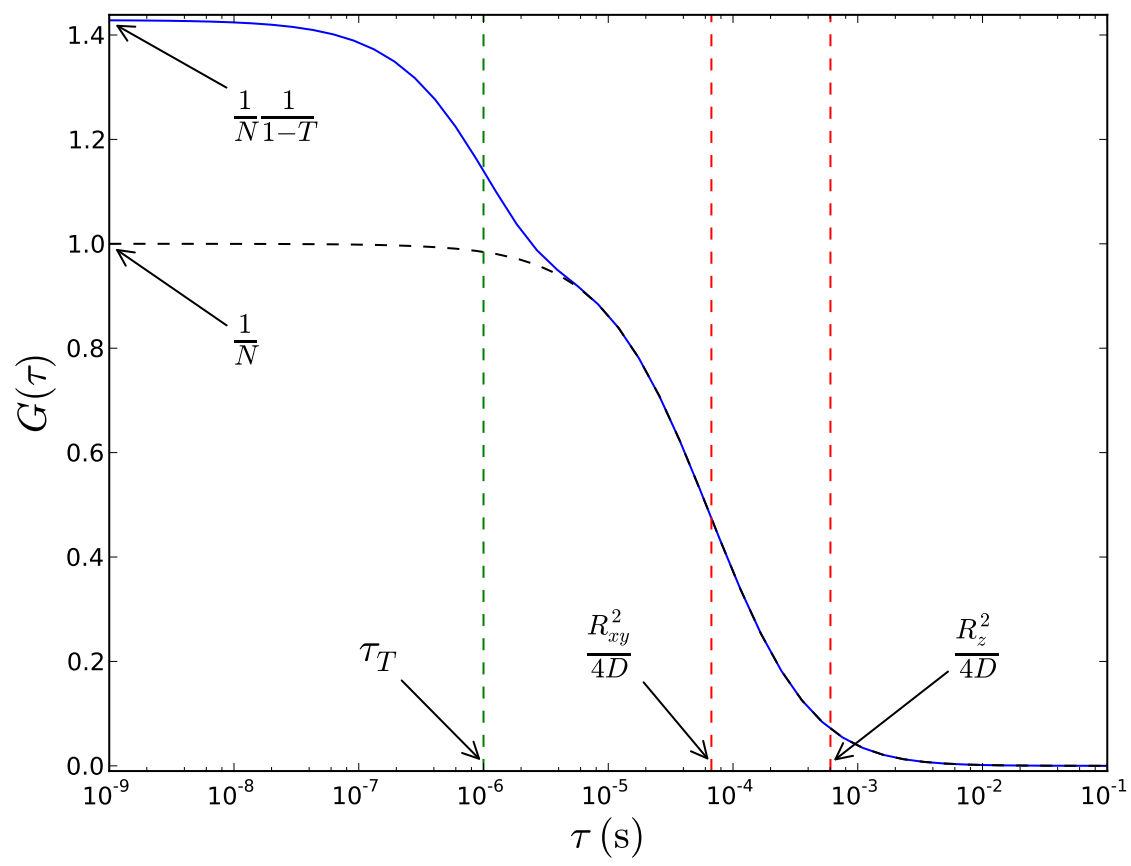

Figure 3.9.: Autocorrelation of the fluorescence intensity of molecules in a solution diffusing in a Gaussian beam with an excitation profile of the form of equation 3.34 and enduring transitions in the triplet state. This curve results from equation 3.57 and features additional fluctuations in the fast time regime compared to the curve of figure 3.8. $\tau_{T}$ is the triplet or bunching time and $N(1-T)$ is the mean number of molecules being in either one of the singlet states. The dark dashed line corresponding to equation 3.53 was also sketched for comparison.

A common problem in FCS measurements when measuring diffusion coefficients of molecules is the presence of noise that is superimposed on the fluorescence signal of the molecules. For FCS measurements in solution the most common source of noise is Raman scattering of water molecules. This noise is uncorrelated and consequently does not cause any fluctuations. But the overall amplitude of the autocorrelation of the total signal (noise + fluorescence) $G_{\mathrm{TOT}}(\tau)$ is diminished in 
comparison to the autocorrelation of the fluorescence signal alone $G_{\mathrm{f}}(\tau)[20]$ :

$$
G_{\mathrm{TOT}}=\left(1-\frac{I_{\mathrm{B}}}{I_{\mathrm{TOT}}}\right)^{2} G_{\mathrm{f}}(\tau)
$$

where $I_{\mathrm{B}}$ is the background intensity superimposed on the fluorescence intensity $I_{\mathrm{f}}$ leading to a total intensity $I_{\mathrm{TOT}}=I_{\mathrm{B}}+I_{\mathrm{f}}$.

The scheme of gaining information on the dynamical properties of fluorescent molecules (and above all fluorescent labelled molecules) diffusing through a volume of defined excitation profile has made FCS a widespread technique particularly in the field of biophysics. The inverted scheme, i.e. the determination of the excitation profile with a fluorescent molecule of defined diffusion coefficient is naturally also possible and usually applied for calibration of the size of a Gaussian beam. Similarly to this inverted scheme, FCS was used in this work to gain information on the excitation volume at the tip of a near-field optical probe (chapter 7). Since no analytical description of the near-field of a real near-field optical probe is possible, a three steps process has to be adopted. First, modeling of the excitation profile has to be carried out. Then, this profile is fed to a Monte-Carlo simulation to simulate the fluctuations of intensity stemming from several molecules of defined photophysical parameters and dynamical properties. Finally, the result of this simulation has to be compared with the experimental autocorrelation and the best match is assumed to give the simulated excitation profile describing best the experimental simulation profile. The main features of these modelings are presented in the next chapter. 



\section{Modeling of the optical near-field distribution at NSOM probes using FCS}

In the previous chapter, it was shown that the diffusion of single fluorescent particles in solution through a volume illuminated by a given intensity distribution gives rise to correlation in the fluorescence signal depending on the diffusion coefficient of the particles and the dimensions of the volume (section 3.2.2). The analytical derivation of the autocorrelation function was possible and consequently the volume dimensions can be calculated from this function. However, for more complex intensity distributions, there is in general no analytical solution for the autocorrelation function. Therefore, the retrieval of the intensity distributions from the measured autocorrelation function should ideally be conducted in two steps. Firstly, assume a certain intensity distribution in a solution with diffusing particles, then simulate the fluorescence signal that would be obtained and calculate the corresponding autocorrelation function. Secondly, fit the simulated autocorrelation function to the one measured in the experiment. This procedure has to be repeated with varying intensity distributions until the best fit is obtained. The first step is the modeling part and is the topic of this chapter.

\subsection{Principles of modeling}

\subsubsection{Parameters of simulation}

\section{Fixed particle}

To model the photon emission of a fluorescent particle under constant illumination, we can use the 3-level system presented in section 3.1.2. In this simplification of the energy level diagram of a fluorescent particle and for an excitation light of given intensity $I$ and frequency $\nu$, only a small number of parameters describe entirely the photophysics of the particle. These are the absorption cross-section $\sigma_{\text {abs }}$ and the transition rates $k_{21}, k_{23}$ and $k_{31}$ between the different energy levels.

To further model the time dependence of the photon emission, it is important to stress the stochastic nature of the different processes involved in the emission, i.e. the transitions from one energy level to another. The mean number of transition events occuring in a given interval of time is fixed and given by the transition rates. Since these events occur independently of time, the stochastic process of 
transition between two energy levels can be modelled with a Poisson distribution. This probability distribution reads:

$$
p(X=N ; k \Delta t)=\frac{(k \Delta t)^{N} \mathrm{e}^{-\mathrm{k} \Delta \mathrm{t}}}{N !}
$$

where $N$ is the number of transition events expected in a given interval of time $\Delta t$ and $k$ is the transition rate. This probability distribution is also sketched in figure 4.1 .

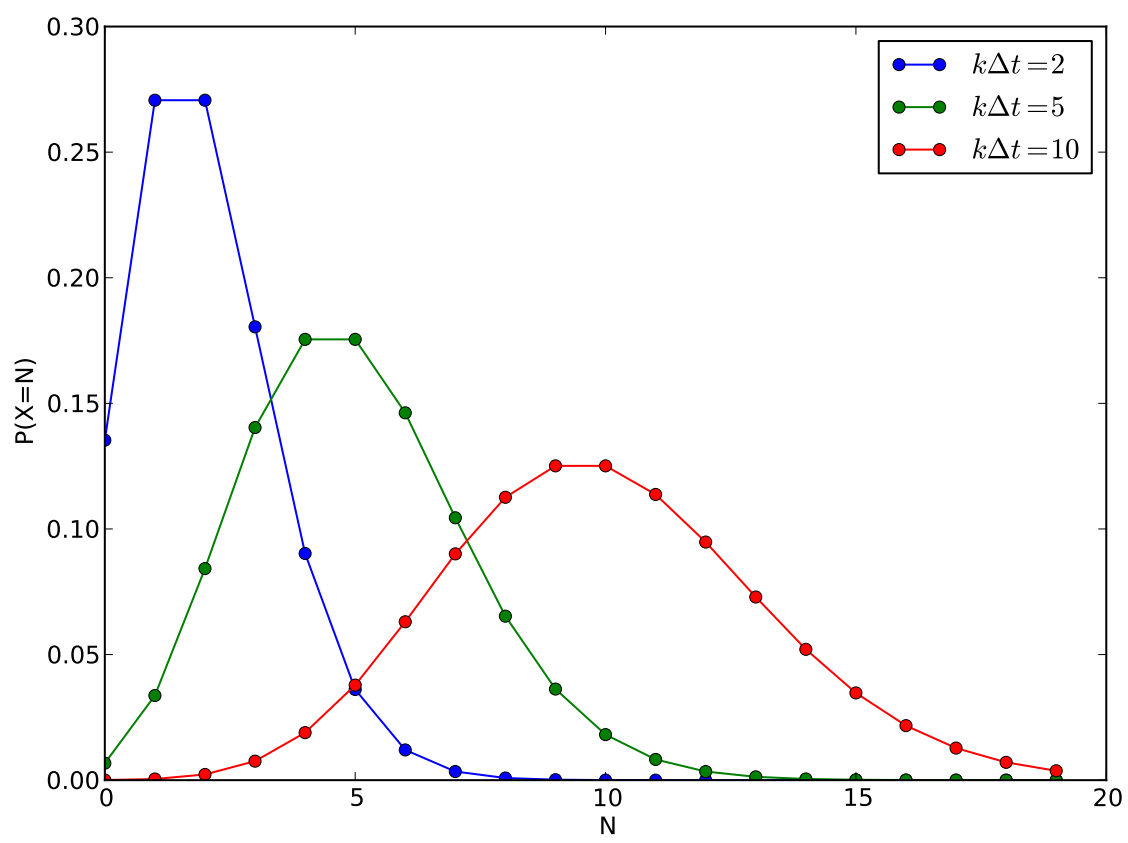

Figure 4.1.: Probability mass function of the Poisson distribution for three different average numbers of events in a given time interval $\Delta t$. The number of events expected to occur with the highest probability in this time interval is equal to the average count $k \Delta t$.

For the simulation of a fluorescence time trace the fact that the number of transition events in a given interval follows a Poisson distribution with mean value $k \Delta t$ has an important consequence: the time between two transitions follows an exponential distribution with rate parameter $k$ (see for example [67]):

$$
p(t, k)=k \mathrm{e}^{-k t}
$$

where $p(T=t, k)$ represents the probability density function for the random variable $T$ and the probability to have a transition at time $t_{1}$ after the last transition is given by $\int_{0}^{t_{1}} p(t, k) \mathrm{d} t$. Not surprising is that the expected value of the random variable $\mathrm{T}$ is $1 / k$. 
This result enables one to efficiently simulate the transition events in the particle by drawing the random time between two events from an exponential distribution with rate parameters equal to the transition rates $k_{12}, k_{21}, k_{23}$ and $k_{31}$. The three last mentioned are intrinsic to the particle, whereas the first one also depends on the intensity and the wavelength of the excitation light (see equation 3.1).

With this result, the simulation of the fluorescence emission of the particles is straightforward. At time $t=0$ the particle is said to be in the ground state. Wavelength and intensity of the excitation light and the absorption cross-section of the particle define $k_{12}$. Therefore, the time $t_{1}^{12}$ after which the particle is excited in the singlet state, is drawn from an exponential distribution with rate parameter $k_{12}$ using conventional packages to generate random numbers with known probability distribution. In the excited singlet state, the particle can either relax in the singlet ground state or the triplet ground state. To decide through which pathway the particle would go a time $t_{1}^{21}$ is drawn from an exponential distribution with rate parameter $k_{21}$ and another one, $t_{1}^{23}$, from the same distribution but with rate parameter $k_{23}$. If $t_{1}^{21}<t_{1}^{23}$ then the molecule has relaxed in the singlet ground state before an intersystem crossing could occur. Since the transition $2 \rightarrow 1$ is related to a photon emission, a photon time arrival is recorded: $t_{1}^{12}+t_{1}^{21}$. If $t_{1}^{23}<t_{1}^{21}$ then the molecule relaxes in the triplet ground state and will not come back to the singlet ground state before a time $t_{1}^{31}$ drawn from an exponential distribution with rate parameter $k_{31}$.

The effective emission of a photon when the particle is back to the singlet ground state upon relaxation from the singlet excited state, can additionally be controlled by defining a probability of emission: this enables one to model the quantum yield of a particle. In this case, after relaxation from the singlet excited state $\left(t_{1}^{21}<t_{1}^{23}\right)$, a number is drawn from a geometric distribution (basically a 0 or 1 reflecting the failure or the success of a Bernouilli trial). In case of success, the photon is emitted.

Additionally, the quality or quantum efficiency of the detection setup effect can be taken into account after emission of the photon. This is also modeled by drawing random numbers from a geometric distribution and counting only the photon as detected in case of success.

When the particle is back to its ground state (either after $t_{1}^{12}+t_{1}^{21}$ upon photon emission or after $t_{1}^{12}+t_{1}^{23}+t_{1}^{31}$ following an intersystem crossing) and only in this case, the particle can be excited again. Therefore, it has to be checked if at a time $t_{1}^{12}+t_{2}^{12}$ with $t_{2}^{12}$ giving the arrival time of the next exciting photons, the particle is already back to the singlet ground state. If it is not the case, this photon will not contribute to the excitation of the particle and the time arrival $t_{3}^{12}$ of the next photon is computed until the particle is excitable and the whole process is repeated.

\section{Diffusing particles}

In case of diffusing particles, several new parameters compared to the case of a fixed particle are to be taken into account. First, to avoid computation time to increase beyond reasonable values, a simulation volume is defined, i.e. the volume 
in which the particles are allowed to diffuse freely. Since the intensity distributions of the simulated fields often have a symmetry axis, e.g. $z$, the axis $x$ and $y$ play a symmetric role. Consequently, the simulation volume is set to be all $(x, y, z)$ so that $(|x|,|y|,|z|)<\left(L_{x}, L_{y}, L_{z}\right)$ with $L_{x}=L_{y}$. If a particle makes a movement that would bring it over the boundaries of the volume, some boundary conditions are applied. In case of an open boundary, a periodic boundary is applied to the coordinate(s) above the limits. For example, if $x>L_{x}$ then the new coordinate $x_{N}$ of the particle is defined to be $x_{N}=x-2 L_{x}$ i.e. the particle appears on the opposite side of the simulation volume. In case of a closed boundary, like a glass surface, a reflective boundary is applied: $x_{N}=2 L_{x}-x$ as if the particle would hit the surface and come back (see figure 4.2).

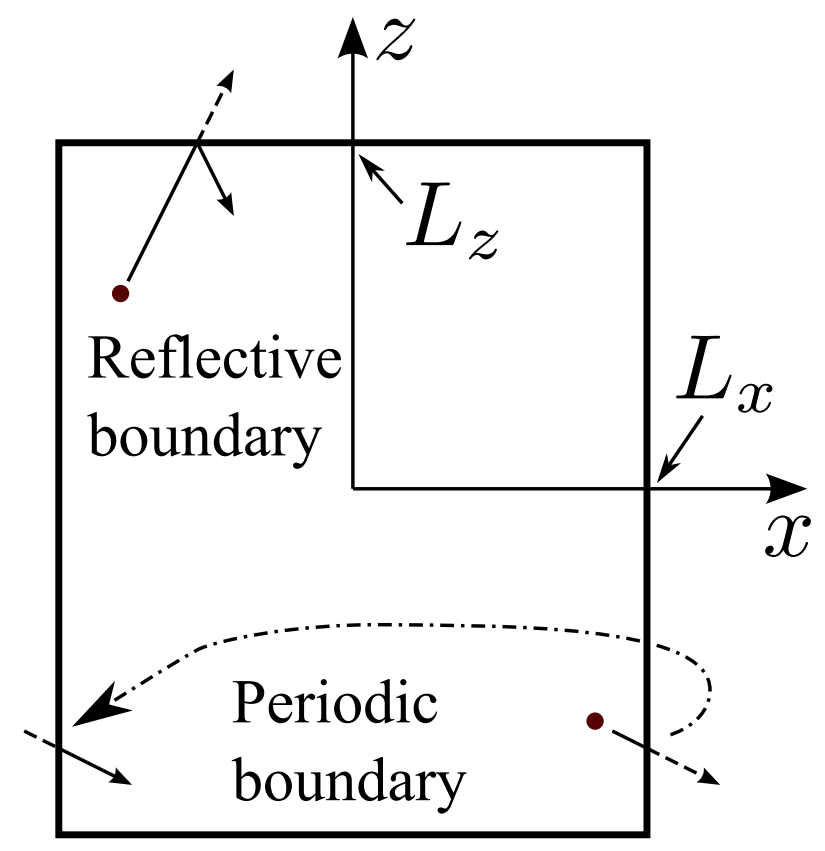

Figure 4.2.: Definition of the simulation volume in the case of diffusing particles. If a particle comes to a movement that would bring it over the boundary of this volume, then boundary conditions are applied. Two kinds of boundary conditions are simulated: periodic boundary, if the boundary is open (water for example) or reflective boundary, if the boundary is close (glass or metal for example).

Similarly to transitions between energy levels, the diffusion of particles in the volume is not a deterministic process but a stochastic process. Its probability distribution is different though and is given upon solving the diffusion equation given in equation 3.30 and rewritten as a stochastic differential equation:

$$
\frac{\partial p(\vec{r}, t)}{\partial t}=D \nabla^{2} p(\vec{r}, t)
$$

where $p(\vec{r}, t)$ is the probability density function of the continuous random variable 
$\vec{R}$. From the perspective of probability density functions, this equation is derived by using the so-called "master equation" that describes the time evolution of the density probability function. This leads to the Fokker-Planck equation and under the assumption of no external forces on the particles, this equation reduces to the above diffusion equation (see for example [68]).

The solution of this equation, i.e. the density probability function describing the movement of a particle from a point $\vec{r}$ at time $t$ to a point $\vec{r}+\Delta \vec{r}$ at time $t+\Delta t$ can be decomposed in three density probablity functions, each acting on one of the coordinates $x, y$ and $z$. For example in the case of the coordinate $x$ [68]:

$$
p(\Delta x, \Delta t)=\frac{1}{\sqrt{4 \pi D \Delta t}} \mathrm{e}^{-\frac{|\Delta x|^{2}}{4 D \Delta t}}
$$

This is a Gaussian distribution with mean value $\langle\Delta x\rangle=0$ and standard deviation $\sigma=\left\langle\Delta x^{2}\right\rangle=\sqrt{2 D \Delta t}$ from which random number can easily be drawn using standard random number generation packages. The dependence over $\Delta t$ of this probability density function requires the introduction of a time step $T_{S}$ for the simulation of the movement of the particles. At each time step, the new positions of the particles are calculated from the random number $\Delta x, \Delta y$ and $\Delta z$ drawn from a gaussian distribution with zero mean and standard deviation $\sqrt{2 D T_{S}}$.

At this point it is important to note that the diffusion mechanism imposes some constraints on the simulation volume in order to avoid artificial correlations. This problem stems from the fact that with periodic or reflective boundary conditions, the molecules inside the volume have a greater probability to come back at some point, which would not be the case without the boundaries. In the one-dimensional case for a molecule at a position $x$ at time $t=0$ the variance $\left\langle x^{2}(t)\right\rangle$ increases with time as $\sqrt{2 D t}$. Consequently, for an infinitely extended volume, equation 4.4 states that $p(\Delta x, \Delta t) \rightarrow 0$ for $\Delta t \rightarrow \infty$. This means that, after a sufficiently long time, it is impossible to exactly locate the molecule, i.e. the steady state value of $p(\Delta x, \Delta t)$ is $\bar{p}=0$. For a finite volume, however, $\bar{p}$ has a finite value, different from 0 . Therefore, $G(\tau) \neq 0$ for every $\tau=\Delta t$. As a consequence, the simulation volume has to be chosen, so that $p(\Delta x, \Delta t)$ reaches its steady state value $\bar{p}$ only for values of $\tau$, for which $G(\tau)$ practically reached its minimal value.

The last important difference between the simulation of the fluorescence emission of diffusing particles and that of fixed particles is the dependence of the photon excitation rate $k_{12}$ on the spatial position of the particle. To compute this rate, either the intensity distribution is known analytically as in the case of a Gaussian distributed intensity or the intensity distribution is itself computed by numerical methods. In the last case, the intensity is usually only calculated at discrete points of the simulation volume. If these points are sufficiently close to one another and distributed over a regular grid, then a trilinear interpolation scheme can be used to get the intensity at an arbitrarily point $(x, y, z)$. Either way the intensity distribution can be precisely mapped and the spatial dependent photon excitation rate $k_{12}(x, y, z)$ is obtained by multiplying this intensity by the constant factor $\sigma_{\text {abs }} / h \nu$ of equation 3.1 . 
In practice the simulation starts by giving the number of molecules used in the simulation volume. Then all molecules are given a random position, here again, using random numbers, but this time drawn from a uniform distribution over the half-closed interval $[0,1)$ that are then scaled to the dimensions of the simulation volume. Then, at each step and for each molecule, a list of relative photon arrival times $\left\{t_{i, r}^{12}\right\}$ is drawn from an exponential distribution with rate parameter the local photon excitation rate $k_{12}(x, y, z)$. The list of absolute photon arrival times is then simply built up by $\left\{\sum_{i=1}^{j} t_{i, r}^{12} \quad j \in[1, n]\right\}=\left\{t_{i}^{12}\right\}$ where $n$ is the last relative photon arrival time for which $\sum_{i=1}^{n} t_{i, r}^{12}$ is smaller than the time step $T_{S}$. For each value $t_{i}^{12}$ the same process as in the case of fixed particles is carried out, i.e. comparison of $t_{i}^{21}$ and $t_{i}^{23}$ leading to a photon emission at $t_{i}^{12}+t_{i}^{21}$ or intersystem crossing with subsequent return to the singlet ground state at $t_{i}^{12}+t_{i}^{23}+t_{i}^{31}$. A new excitation by the $j^{\text {th }}$ following photon is then only possible if $t_{j}^{12}>t_{i}^{12}+t_{i}^{23}+t_{i}^{31}$. Additionally, for each simulation step, only photon emission times $t_{i}^{12}+t_{i}^{21}<T_{S}$ are taken into account.

\section{Parameters specific to the near-field aperture probe}

For the specific simulation of fluorescence emission of particles diffusing in the intensity distribution at the near-field probe, two additional processes were taken into account to give simulation results as close as possible from the experimental results. The first process is the decrease in fluorescence emission near metal interfaces where energy from the particles is transmitted to the free electrons of the metal through a non radiative process. This fluorescence quenching is also well known in the field of Fluorescence Resonance Energy Transfer (FRET) [69]. However, in this case, the energy transfer takes place between two particles and not a particle and the metal. Additionally, the fluorescence of the first particle transmitting its energy to the second particle is quenched, yet, the second particle fluoresces. Even if these differences exist the energy transfer process can be reasonably thought as to be similar. How much energy is transferred in this process reflects the so-called FRET efficiency $E$ :

$$
E=\frac{1}{1+\left(\frac{|\vec{r}|}{R_{0}}\right)^{6}}
$$

where $|\vec{r}|$ is the distance between the energy donor and the energy acceptor and $R_{0}$ is the Förster distance, at which the energy transfer efficiency is $50 \%$.

To model this energy transfer, a spatially dependent probability for a particle relaxing from the singlet excited state into the singlet ground state by emitting a photon is computed. In the case of no quenching this probability is 1: the transition $2 \rightarrow 1$ leads always to the photon emission. In the case of quenching, this probability is $(1-E)$, where $E$ is the quantum efficiency of the energy transfer. In the case of FRET, $E$ is simply the FRET efficiency. To give a bit more flexibility to the quenching behavior at the metallic surface an additional parameter $\overrightarrow{r_{0}}$ pointing at a position inside the metal is introduced so that the FRET efficiency is not 
necessarily 1 at the surface. The emission probability $p_{E}(\vec{r})$ of a photon after the transition $2 \rightarrow 1$ is consequently given by:

$$
p_{\mathrm{Em}}^{\mathrm{F}}(\vec{r})=\frac{1}{1+\left(\frac{\left|\vec{r}-\overrightarrow{r_{0}}\right|}{R_{0}}\right)^{-6}}
$$

Another quenching model can also be envisaged similar to the analytical form of the Förster energy transfer. This model called "exponential decay" (whereas the first quenching model is called a "Förster decay") allows to construct an arbitrarily steep quenching profile centered on an arbitrary position $\overrightarrow{r_{1 / 2}}$ away from the metal can be constructed. It is decribed through another spatial dependent photon emission probablity:

$$
p_{\mathrm{Em}}^{\mathrm{E}}(\vec{r})=\frac{1}{1+\mathrm{e}^{-\alpha\left|\vec{r}-r_{1 / 2}\right|}}
$$

In figure 4.3 some examples of quenching profiles using a Förster decay and an exponential decay are shown. The problem of modeling a steep decay in the quenching profile using a Förster decay is due to the flexible definition used in equation 4.6: in the case of a one dimensional quenching profile for example, if the distance $\Delta x$ for which the probablity $p_{\mathrm{Em}}^{\mathrm{F}}(x)$ must fall from $90 \%$ to $10 \%$ is chosen too small, and the distance $x_{1 / 2}$ at which this probablity is 1 is too large, then the value $x_{0}$ defined in equation 4.6 will be located outside the metal. The result is a physically meaningless minimum of $p_{\mathrm{Em}}^{\mathrm{F}}(x)$ away from the metal interface in the solution.

In addition to the quenching behavior of the fluorescence of the particles at the metal interface, a further parameter was used in the simulation to describe the possiblity for the particle to bind for some time to the glass surface of the aperture probe or (in an exclusive sense) to the metal surface. To model this binding behavior, a distance $d$ to the binding surface was first defined. For distances of the particles greater than $d$ the particles diffuse freely in the solution. But for each time step $T_{S}$ where a particle is at a distance of the surface smaller than $d$, the probability distribution that the particle binds to the surface is considered to be a geometric distribution with a probability of success (here called "binding probability") defined before the begin of the simulation. This kind of distribution is also represented in usual random number generation packages, so that a number drawn from this distribution directly reflects if the binding takes place or not.

\subsubsection{Accuracy of the model}

To test the validity of the model presented in the precedent section, simulation results were compared to situations where an analytical solution exists for the autocorrelation function. 


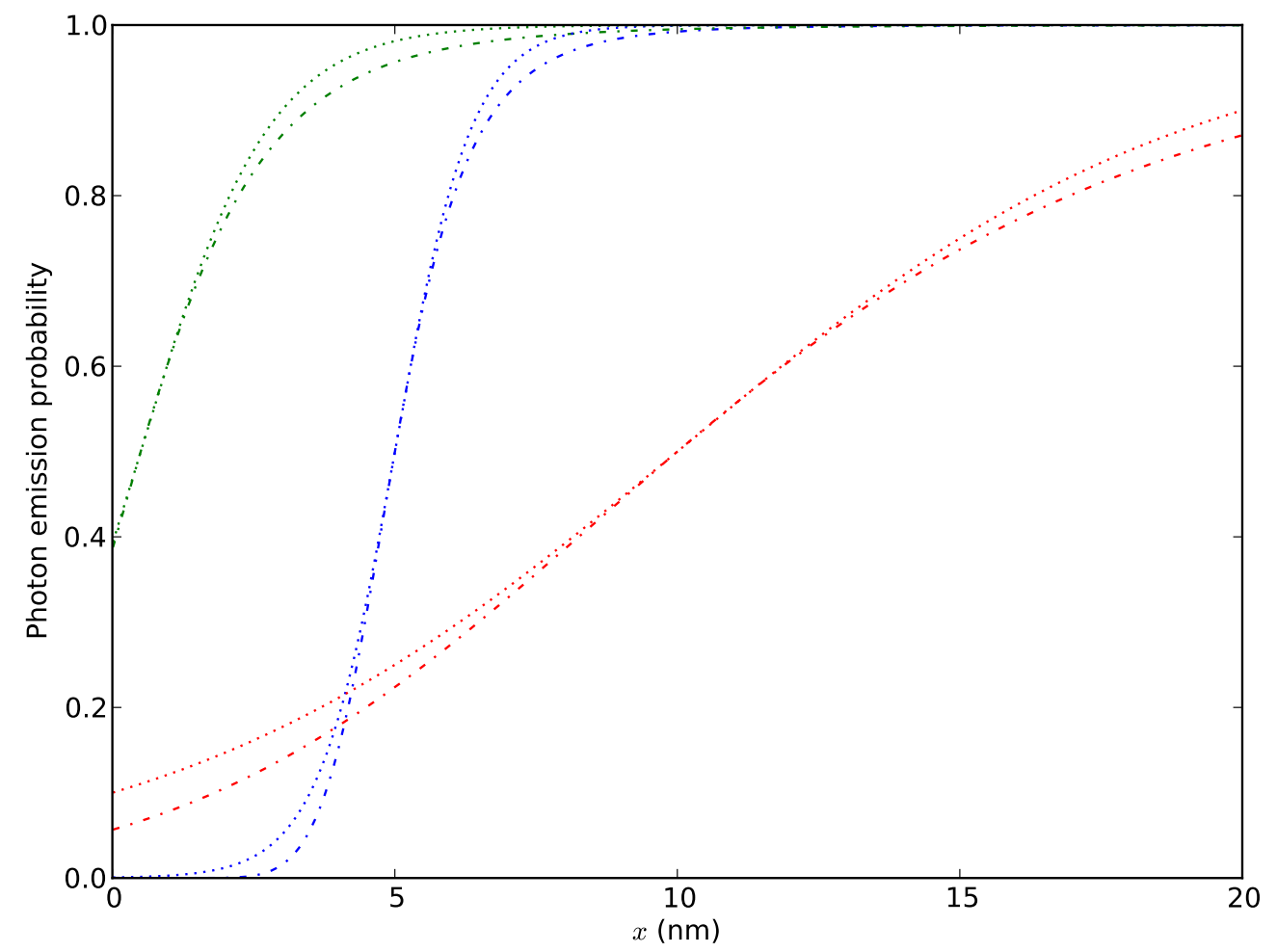

Figure 4.3.: One dimensional Förster (dash dotted line) and exponential (dotted line) decaying quenching profiles at a distance $x$ of the metal surface and for different parameters $\overrightarrow{r_{0}}=x_{0}, R_{0}, \alpha$ and $\overrightarrow{r_{1 / 2}}=x_{1 / 2}$. The parameters were chosen so that the values of $x$ at which the probablity of photon emission decreases to 0.5 and the distance $\Delta x$ for which this probability fall from 0.9 to 0.1 is the same for curves of same color. Blue curves: $x_{1 / 2}=5 \mathrm{~nm}, \Delta x=3 \mathrm{~nm}$. Red curves: $x_{1 / 2}=10 \mathrm{~nm}, \Delta x=$ $20 \mathrm{~nm}$. Green curves: $x_{1 / 2}=0.5 \mathrm{~nm}, \Delta x=5 \mathrm{~nm}$. It has to be noted that the blue dash dotted curve does not decrease linearly to 0 when $x \rightarrow 0$ but the minimum is for a value of $x$ slightly greater than 0 , which has no physical meaning. For this reason, steep quenching profiles at some distance away from the metal surface cannot be modeled that well with the Förster decay. The exponential decay addresses this issue. 


\section{Fixed particle}

In the case of fixed particles the result of a typical simulation was already presented in section 3.2.1 and the corresponding analytical result for the autocorrelation function was given in equation 3.29 :

$$
G(\tau)=-\left(1+\frac{k_{12} k_{23}}{k_{31}\left(k_{12}+k_{21}\right)}\right) \mathrm{e}^{-\left(k_{12}+k_{21}\right) \tau}+\frac{k_{12} k_{23}}{k_{31}\left(k_{12}+k_{21}\right)} \mathrm{e}^{-\left(k_{31}+\frac{k_{12} k_{23}}{k_{12}+k_{21}}\right) \tau}
$$

The simulation was done using the photophysical parameters of Rhodamine6G $(\mathrm{Rh} 6 \mathrm{G})$ given in section 3.1.2, i.e. $\sigma_{\mathrm{abs}, \mathrm{Rh} 6 \mathrm{G}}=170 \cdot 10^{-18} \mathrm{~cm}^{2}, k_{21}=250 \cdot 10^{6} 1 / \mathrm{s}$, $k_{23}=1.1 \cdot 10^{6} 1 / \mathrm{s}, k_{31}=0.5 \cdot 10^{6} 1 / \mathrm{s}$, a quantum yield of 0.95 and a detection probability of $10 \%$. The calculated autocorrelation of the fluorescence time trace obtained from the simulation are shown together with the analytical autocorrelation function in figure 4.4 for different excitation intensities.

The perfect agreement of the calculated autocorrelation from the simulation and the theoretical autocorrelation curve is the best confirmation that the simulation model reflects very well the results predicted from section 3.1.2. An additional confirmation can be found by considering the count rate of detected photons, that was given in equation 3.15 and calculated with equation 3.10. These count rates are $2.73 \cdot 10^{6} 1 / \mathrm{s}, 6.33 \cdot 10^{6} 1 / \mathrm{s}$ and $7.17 \cdot 10^{6} 1 / \mathrm{s}$ for the intensities 100,1000 and $5000 \mathrm{~kW} / \mathrm{cm}^{2}$, respectively. All the values of the simulations correspond to the theoretical values within less than $1 \%$.

\section{Diffusing particles}

The validity of the simulation model for the diffusion of particle in solution illuminated with a given intensity distribution was tested in two cases, where an analytical solution is known for the autocorrelation function of the fluorescence signal.

For these simulations, the photophysical parameters $\left(\tau_{f}, Q, \sigma_{\mathrm{abs}}, k_{21}, k_{23}\right.$ and $k_{31}$ ) and the diffusion coefficient $D$ of Atto655 additionally to those of Rh6G were used. The high absorption cross-section was already introduced in section 3.1.2. Contrary to Rh6G, Atto655 does not show any triplet excitation at high intensities [70]. For this reason its triplet excitation rate $k_{23}$ is set to 0 and consequently $k_{31}$ does not need to be given. The necessary data for these two molecules used in the following simulation are listed in table 4.1.

In the following simulations, the fit of the correlation functions can be compared to the theoretical values. For the sake of clarity, the relations between these theoretical values and the values than can be extracted from the fits are recapitulated in table 4.2 together with the references where these relations appear in chapter 3 .

The first tested model was detailed in section 3.2.2. This is the diffusion of particle through a focussed Gaussian beam. Simulations were first carried out with the parameters of Atto655 in order to avoid triplet excitation and stick to the simple model that leads to equation 3.53. An example of such a simulation is given 


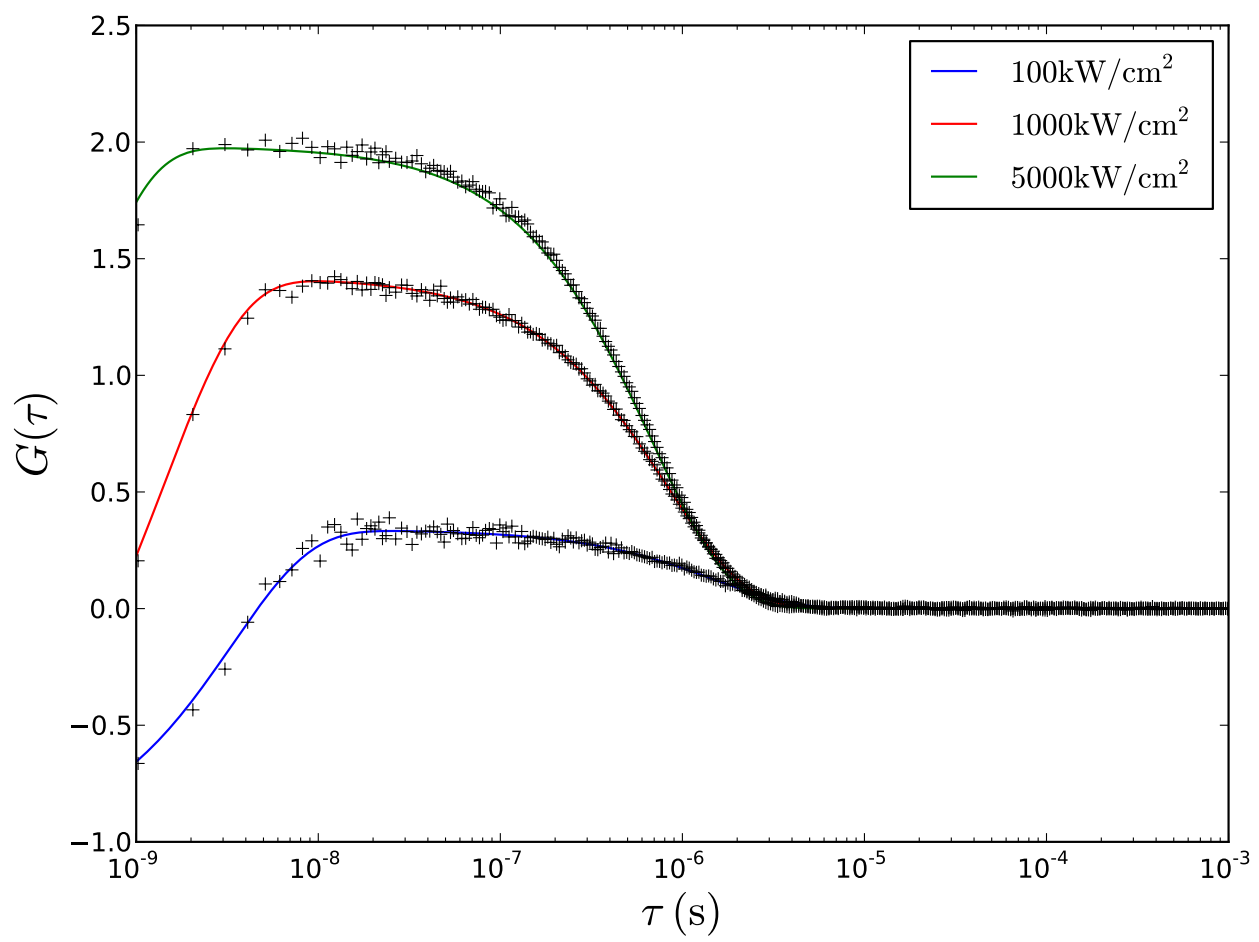

Figure 4.4.: Exact autocorrelation function of a fixed particle illuminated under different excitation intensities (continuous line) and the corresponding calculated autocorrelation from the simulation described in the precedent section (crosses). The simulation duration is $100 \mathrm{~ms}$ for the three different excitation intensities of 100,1000 and $5000 \mathrm{~kW} / \mathrm{cm}^{2}$. The detected photon rates are for these intensities $2.73 \cdot 10^{6} 1 / \mathrm{s}, 6.36 \cdot 10^{6} 1 / \mathrm{s}$ and $7.12 \cdot 10^{6} 1 / \mathrm{s}$, respectively. This is a non linear dependence on the excitation intensity showing that the fluorescence emission of the particle is at $1000 \mathrm{~kW} / \mathrm{cm}^{2}$ already highly saturated. 


\begin{tabular}{|c|c|c|}
\hline Parameter & $\begin{array}{c}\text { Atto655 } \\
\text { (for } \lambda=640 \mathrm{~nm})\end{array}$ & $\begin{array}{c}\text { Rh6G } \\
\text { (for } \lambda=532 \mathrm{~nm})\end{array}$ \\
\hline$\tau_{f}(\mathrm{~ns})$ & 1.8 & 4.2 \\
$\mathrm{Q}$ & 0.3 & 0.95 \\
$\sigma_{\mathrm{abs}}\left(\mathrm{cm}^{2}\right)$ & $315 \cdot 10^{-18}$ & $170 \cdot 10^{-18}$ \\
$k_{21}\left(\mathrm{~s}^{-1}\right)$ & $1850 \cdot 10^{6}$ & $250 \cdot 10^{6}$ \\
$k_{23}\left(\mathrm{~s}^{-1}\right)$ & 0 & $1.1 \cdot 10^{6}$ \\
$k_{31}\left(\mathrm{~s}^{-1}\right)$ & - & $0.5 \cdot 10^{6}$ \\
$D\left(\mu \mathrm{m}^{2} / \mathrm{s}\right)$ & 426 & 414 \\
\hline
\end{tabular}

Table 4.1.: Comparative table of values for the fluorescence lifetime $\tau_{f}$, the fluorescence quantum yield $Q$ and the absorption cross-section $\sigma_{\text {abs }}$ of Atto655 and Rh6G. The numerical values for Atto655 for $\tau$ and $Q$ are taken from [60]. The absorption cross section of Atto655 is calculated with the extinction coefficient value given for the maximum of absorption of this fluorescent particle, i.e. at $663 \mathrm{~nm}: \epsilon_{663 \mathrm{~nm}}=125000 \mathrm{M}^{-1} \mathrm{~cm}^{-1}$ converted to the absorption cross section at $640 \mathrm{~nm}$ with equation 3.2 and the absorption spectra given by ATTO-TEC GmbH [60]. $k_{21}$ is derived from $Q$ and $\tau$ using equation 3.14. The photophysical parameters of Rh6G were introduced in section 3.1.2. The diffusion coefficients were taken from [71] (Atto655) and [72] (Rh6G). 


\begin{tabular}{|c|c|c|c|c|}
\hline & & $N_{\mathrm{TOT}}$ & \multirow{2}{*}{$C_{0}=\frac{N_{\mathrm{TO}}}{\left(2 L_{x y}\right)^{2} \cdot 2 L_{z}}$} & \multirow{3}{*}{$N=C_{0} \cdot V_{\mathrm{exc}}$} \\
\hline & & $L_{x y}, L_{z}$ & & \\
\hline & & $R_{x y}, R_{z}$ & \multirow[t]{2}{*}{$V_{\mathrm{exc}}=\pi^{3 / 2} R_{x y}^{2} R_{z}$} & \\
\hline & & $D$ & & \\
\hline \multirow{3}{*}{$\begin{array}{c}T= \\
\frac{k_{23}}{k_{23}+k_{31}} \frac{I_{T} / I_{S}}{1+I_{T} / I_{S}}\end{array}$} & $I_{T}=I_{0} / 2$ & $I_{0}$ & \multirow{3}{*}{$k_{12}=\frac{\sigma_{\mathrm{abs}} I_{T}}{h \nu}$} & \multirow{4}{*}{$\begin{array}{c}T T= \\
\frac{1}{k_{31}+\frac{k_{12} k_{23}}{k_{12}+k_{21}}} \\
=\frac{1-T}{k_{31}}\end{array}$} \\
\hline & \multirow{3}{*}{$\begin{array}{c}I_{S}= \\
I_{T} \frac{k_{31}\left(k_{21}+k_{23}\right)}{k_{12}\left(k_{23}+k_{31}\right)}\end{array}$} & $\lambda$ & & \\
\hline & & $\sigma_{\mathrm{abs}}$ & & \\
\hline$=\overline{1+\frac{k_{31}}{k_{12} k_{23}}\left(k_{12}+k_{21}\right)}$ & & $k_{21}, k_{23}, k_{31}$ & & \\
\hline
\end{tabular}

Table 4.2.: The simulation parameters (middle column) and the different parameters that can be derived from them: $C_{0}$ is the molecular concentration (appearing in equation 3.53), $V_{\text {exc }}$ is the excitation volume given from equation 3.54 and $N$ is the mean number of molecule in the excitation volume. The mean intensity $I_{T}$ is the intensity used to evaluate the triplet characteristic of the molecule (equation 3.58). Together with the saturation intensity of the molecule (equation 3.12) it yields the mean triplet occupation $T\left(\overline{x_{3}}\right.$ in equation 3.11). The triplet time (bunching time) $T T$ is a function of the excitation rate $k_{12}$ (equation 3.1) for the mean intensity $I_{T}$ and is calculated with equation 3.24 where $T T=-1 / s_{2}$ and using the approximation $k_{21}, k_{12} \gg k_{23}, k_{31}$. 
in figure 4.5. Using simulation step of $100 \mathrm{~ns}$, the diffusion of 10 Atto655 molecules were simulated during $60 \mathrm{~s}$ in a $4 \times 4 \times 4 \mu \mathrm{m}^{3}$ volume, which corresponds to a concentration of $\sim 0.26 \mathrm{nM}$. The Gaussian excitation volume was set to be twice as large in the axial direction $(z$ axis) as in the lateral direction $(x, y)$ plane. The correlation function calculated from the simulation was fitted using equation 3.53 and the results of the fit are given in table 4.3. The fit was actually carried out with either two or three free parameters: $N$ and $R_{x y}$ are set as free parameters in both cases and $R_{z}$ is either free or bound to $R_{x y}$ using the relation $R_{z}=S R_{x y}$ with $S=2$.

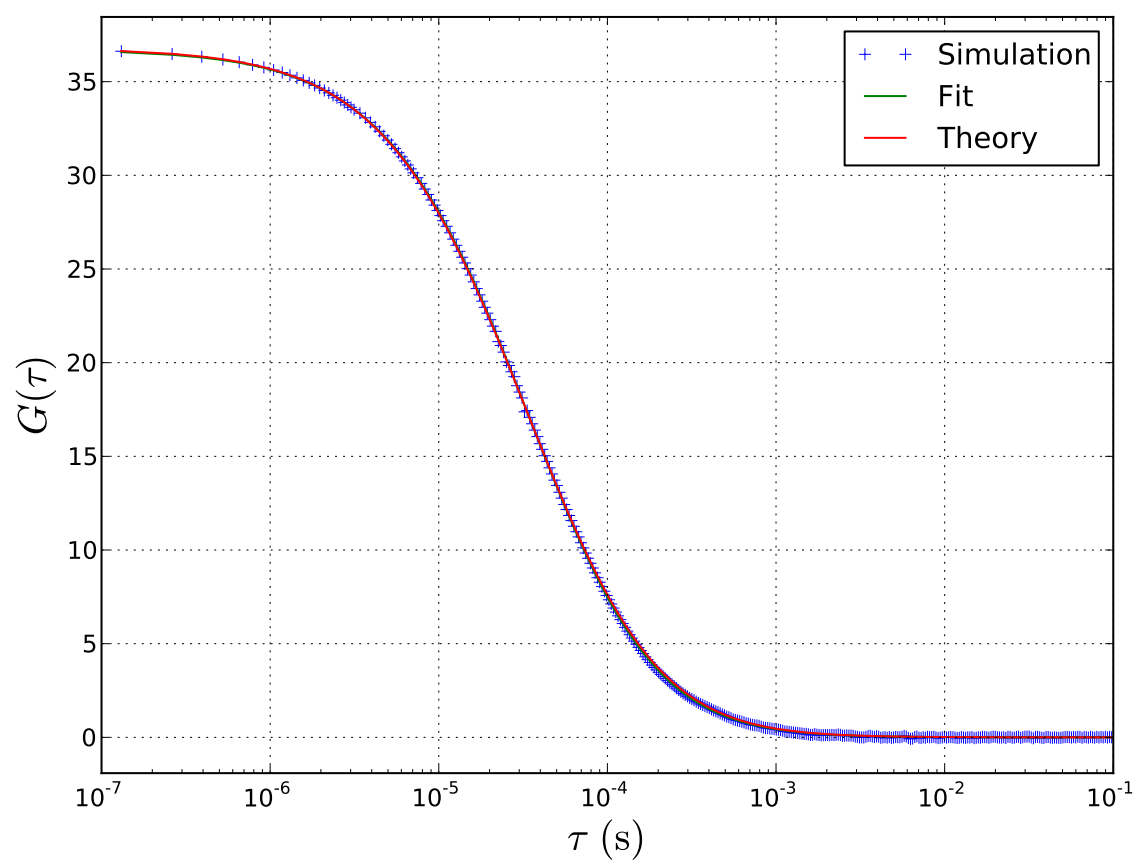

Figure 4.5.: Autocorrelation calculated from a simulation of the diffusion of Atto655 molecules in and out of a focused Gaussian beam (crosses) together with its fit (green curve) to the theoretical curve of equation 3.53. The simulation parameters are $N_{\mathrm{TOT}}=10, L_{x y}=L_{z}=2 \mu \mathrm{m}$, $R_{x y}=250 \mathrm{~nm}, R_{z}=500 \mathrm{~nm}$ and $I_{0}=1000 \mathrm{~kW} / \mathrm{cm}^{2}$. The simulation duration is $60 \mathrm{~s}$. The fit results are presented in table 4.3. Additionally, the theoretical curve corresponding to the simulation parameter is displayed in red.

The fits to the simulated correlation function are obviously very good in both cases for a free or bound parameter $R_{z}$. The standard deviation of the fit with three free fit parameters, is as expected a little better because of the higher number of free parameters. However, looking more precisely at the fit values of table 4.3 shows that fitting with three fit parameters can lead to values somewhat deviating from the simulation values. For example $R_{z}$ deviates from nearly $15 \%$ of its true 


\begin{tabular}{|c|c|c|c|}
\hline \multirow{2}{*}{ Fit parameters } & \multirow{2}{*}{ Theory } & $\begin{array}{c}\text { Fit results } \\
\text { with } R_{z} \\
\text { as free parameter }\end{array}$ & $\begin{array}{c}\text { Fit results } \\
\text { with } R_{z} \text { fixed with } \\
R_{z}=S R_{x y}, S=2\end{array}$ \\
\hline$N$ & $27.19 \cdot 10^{-3}$ & $(27.234 \pm 0.007) \cdot 10^{-3}$ & $(27.14 \pm 0.01) \cdot 10^{-3}$ \\
$R_{x y}(\mathrm{~nm})$ & 250 & $260.0 \pm 0.6$ & $249.1 \pm 0.1$ \\
$R_{z}(\mathrm{~nm})$ & 500 & $425 \pm 3$ & $498.2 \pm 0.2$ \\
\hline
\end{tabular}

Table 4.3.: Comparative table between the values of the free parameters fitted to the autocorrelation curve in figure 4.5 and their theoretical values calculated from table 4.2 .

value. Fitting with only two free fit parameters leads to an error of less than $1 \%$ on $R_{x y}$ (and obviously also on $R_{z}$ ), which is better than the $4 \%$ error for the other fit. The mean value $N$ of molecules in the excitation volume $V=\pi^{3 / 2} R_{x y}^{2} R_{z}$ is also slightly better with less free parameters. This deviation is due to the fact that $R_{x y}$ and $R_{z}$ are nearly linear-dependent parameters, which leads to a large range of possible values for the fit without much disturbing the quality of the fit. Of course, the perfect agreement with the simulation value is conditioned by the knowledge of $S$ but shows that the simulation model accurately describes the simple case of three dimensional diffusion in a Gaussian focused beam.

The simulation gets more complex by including the triplet blinking. This kind of simulation was carried out using the parameters for Rh6G listed in table 4.1 and is presented in figure 4.6. The other parameters of the simulation were left untouched in comparison with the precedent simulation. Notice that here, the excitation volume ( $R_{x y} \sim 150 \mathrm{~nm}$ and $\left.R_{z} \sim 450 \mathrm{~nm}\right)$ is chosen large enough so that the mean diffusion time of the molecules in the excitation volume is much larger than the mean triplet time, which is a condition to derive the equation 3.57. This equation is used to fit the simulation results. The fit values are given in table 4.4. Once again two slightly different fits were carried out: either with $R_{z}$ free or with $R_{z}=S R_{x y}$ and setting $S=2$.

Although the simulated autocorrelation curves can be very well fitted, the fit results deviate somewhat from the expected values. The values for the description of the excitation volume $R_{x y}$ and $R_{z}$ deviate between $5 \%$ and up to $20 \%$ from the simulation data for both free and bound parameter $R_{z}$. Also the mean number of molecule in the excitation volume $N$ and the triplet characteristics $T$ and $T T$ are not satisfying. In fact, this is not due to the simulation model but to the model used to fit the simulation data and described by equation 3.57. Additional simulations show that the errors in the fit values decrease when the excitation intensity maximum $I_{0}$ is decreased. This means that the more the fluorescent molecules are excited in the triplet state, the less accurate is the theoretical model. This is due 


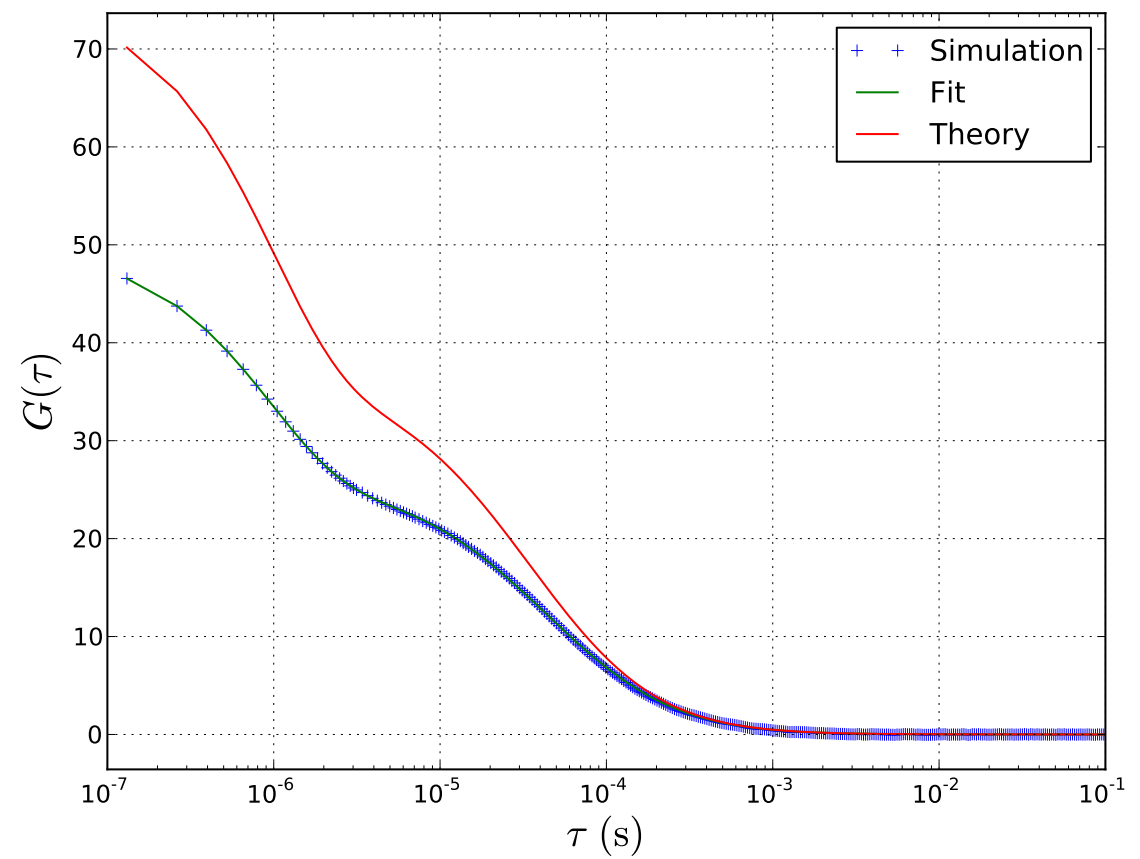

Figure 4.6.: Autocorrelation calculated from a simulation of the fluorescent molecule Rh6G diffusing in and out of a focused Gaussian beam (crosses) together with its fit to the model of equation 3.57 (green curve). In red, the autocorrelation curve expected from the simulation parameters when introduced in equation 3.57. These parameters are $N_{\text {TOT }}=10, L_{x y}=2 \mu \mathrm{m}, L_{z}=2 \mu \mathrm{m}, R_{x y}=250 \mathrm{~nm}, R_{z}=500 \mathrm{~nm}$, and $I_{0}=1000 \mathrm{~kW} / \mathrm{cm}^{2}$. The simulation duration is $60 \mathrm{~s}$.

\begin{tabular}{|c|c|c|c|}
\hline \multirow{2}{*}{ Fit parameters } & Theory & $\begin{array}{c}\text { Fit results } \\
\text { with } R_{z} \\
\text { as free parameter }\end{array}$ & $\begin{array}{c}\text { Fit results } \\
\text { with } R_{z} \text { fixed with } \\
R_{z}=S R_{x y}, S=2\end{array}$ \\
\hline$N$ & $27.19 \cdot 10^{-3}$ & $(38.75 \pm 0.02) \cdot 10^{-3}$ & $(38.46 \pm 0.02) \cdot 10^{-3}$ \\
$R_{x y}(\mathrm{~nm})$ & 250 & $302 \pm 1$ & $281.8 \pm 0,2$ \\
$R_{z}(\mathrm{~nm})$ & 500 & $475 \pm 5$ & $563.6 \pm 0,4$ \\
$T$ & 0.5113 & $0.4805 \pm 0.0004$ & $0.4777 \pm 0.0005$ \\
$T T(\mu \mathrm{s})$ & 0.976 & $0.961 \pm 0.003$ & $0.942 \pm 0.003$ \\
\hline
\end{tabular}

Table 4.4.: Comparative table between the values of the free parameters fitted to the simulated autocorrelation curve of figure 4.6 and their theoretical values calculated from table 4.2 . 
to the fact that at high excitation rate and with a non negligible triplet excitation rate, the fluorescence emission saturates. As a result, the fluorescence emission is no longer proportional to the local excitation intensity and the overall fluorescence emission profile deviate from the Gaussian form of the excitation profile. As a Gaussian profile is needed to derive equation 3.57, the fitting function is not appropriate anymore to describe the fluctuations of the fluorescence signal. It results in fit values trying to approximate the flattened emission profile by a Gaussian profile, which is the source of errors of the model. However, this distortion in the fluorescence emission profile at high excitation intensities can be taken into account by introducing a correction factor [61].

Another type of FCS measurement can be well reproduced with the simulation model: it is the total internal reflection fluorescence (TIRF) experiment [21]. In this kind of experiment, a collimated laser beam is reflected at a glass water interface at an angle larger than the critical angle (see figure 4.7).

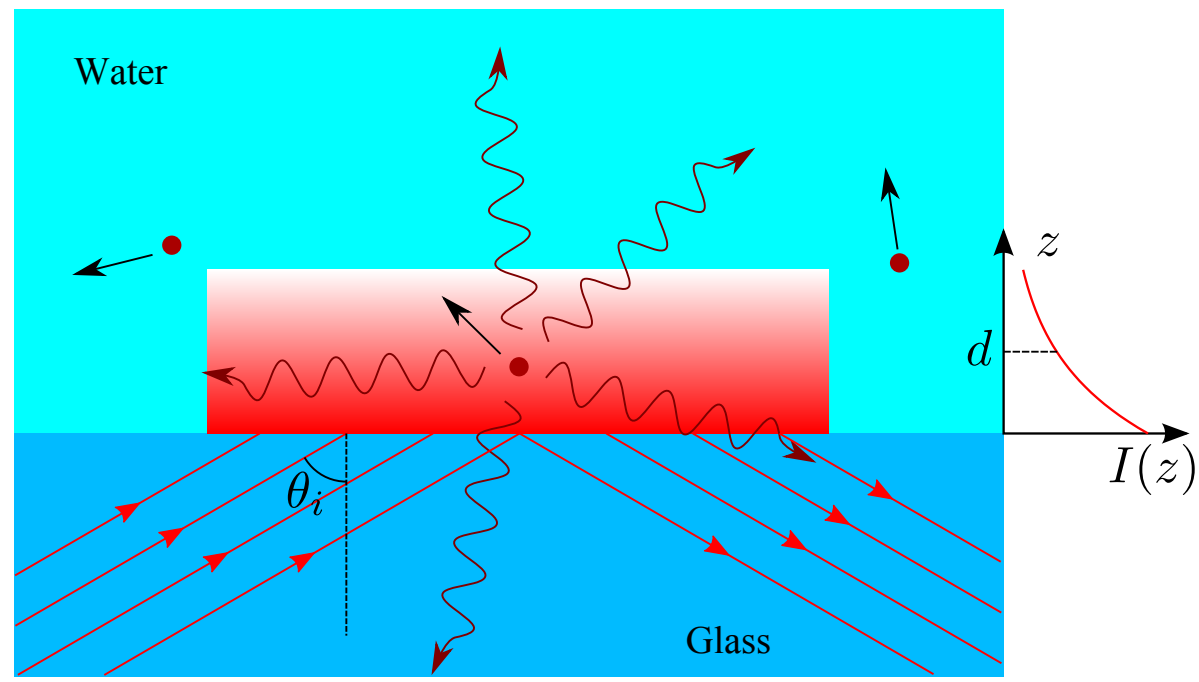

Figure 4.7.: The TIRF setup: a collimated laser beam is reflected at a glass water interface at an angle $\theta_{i}$ larger than the critical angle $\theta_{c}=\sin \left(\frac{n_{\mathrm{W}}}{n_{\mathrm{G}}}\right)^{-1} \sim$ $60^{\circ}$ where $n_{\mathrm{W}}=1.3$ resp. $n_{\mathrm{G}}=1.5$ are the refractive indices of water and glass, respectively. As a result the beam is totally reflected, i.e. no far field propagation of the light can take place in water. However, the light intensity only vanishes exponentially with the distance to the glass water interface and with a characteristic distance $d$ it decays to $1 /$ e.

In this configuration, the excitation light cannot propagate in water (total internal reflection) and only evanescent components of the electromagnetic fields penetrate in this medium. As a result the light intensity in water decreases exponentially with growing distance to the glass water interface. This decrease is characterized by a decay length $d$ at which the intensity $I=I_{0} \mathrm{e}^{-x / d}$ decays to $1 / \mathrm{e}$. The resulting autocorrelation function for the free diffusion of fluorescent molecules in water at 


\begin{tabular}{|c|c|c|}
\hline Fit parameters & Theory & Fit results \\
\hline$N$ & $28 \cdot 10^{-3}$ & $(31.00 \pm 0.02) \cdot 10^{-3}$ \\
$d(\mathrm{~nm})$ & 70 & $70.2 \pm 0.1$ \\
\hline
\end{tabular}

Table 4.5.: Fit results for the FCS simulation of figure 4.8 using equation 4.9.

the glass water interface can also be derived analytically and is given by [73]:

$$
G(\tau)=\frac{1}{N}\left\{\left(1-2 R_{e} \tau\right) w\left[i\left(R_{e} \tau\right)^{\frac{1}{2}}\right]+2\left(\frac{R_{e} \tau}{\pi}\right)^{\frac{1}{2}}\right\}
$$

with the $w$-function (also called "complex error function", "plasma dispersion function" or "Faddeeva function") being defined by [74]:

$$
w(\xi)=\mathrm{e}^{-\xi^{2}} \operatorname{erfc}(-\mathrm{i} \xi)
$$

$N$ represents the mean number of particles in the volume $h^{2} \cdot 2 d$ where $h^{2}$ is the illuminated area in the $(x, y)$ plane (supposing a squared illuminated area and the same detection area) and $R_{e}=D / d^{2}$. Note that here $N$ is defined slightly differently as in [21] where $N$ is defined for a volume of half size: $h^{2} \cdot d$.

To test the validity of the simulation model in the case of a TIRF experiment, a simulation was carried out with a simulation volume with dimensions $L_{x y}=5 \mu \mathrm{m}$, $L_{z}=13 \mu \mathrm{m}$, a total number of molecules of $N_{\mathrm{TOT}}=10$. As the light intensity is homogeneous in the $(x, y)$ plane, the excitation volume $V_{\text {exc }}$ is simply $\left(2 L_{x y}\right)^{2} \cdot 2 d$ and consequently:

$$
N=C_{0} V_{\mathrm{exc}}=\frac{N_{\mathrm{TOT}}}{\left(2 L_{x y}\right)^{2} L_{z}}\left(2 L_{x y}\right)^{2} \cdot 2 d=N_{\mathrm{TOT}} \frac{2 d}{L_{z}}
$$

which is independent of the dimension of the simulation plane $(x, y)$, consistent with the fact that the field distribution is homogeneous in this plane and therefore a diffusion in this plane does not lead to fluorescence fluctuations.

An example of simulation carried on with these parameters is shown in figure 4.8. For the excitation field, a decay length of $d=70 \mathrm{~nm}$ and the observation area in the lateral plane was set to $0.7 \times 0.7 \mu \mathrm{m}^{2}$. These two values are typical ones in TIRF at a glass/water interface [73]. The simulation was carried out with the photophysical and dynamical parameters of Atto655 molecules at an intensity of $1000 \mathrm{~kW} / \mathrm{cm}^{2}$ during $100 \mathrm{~s}$ and with a resolution of $100 \mathrm{~ns}$. The fit, using equation 4.9, is nearly perfect and its results, listed in table 4.5 , show the very strong agreement with the simulation values. For the two values $N$ and $d$, the agreement between simulation and fit is about $10 \%$ and $0.3 \%$, respectively.

To conclude this first section, a model for the simulation of FCS was implemented and it could be shown that it gives accurate results for situations where an analytical 


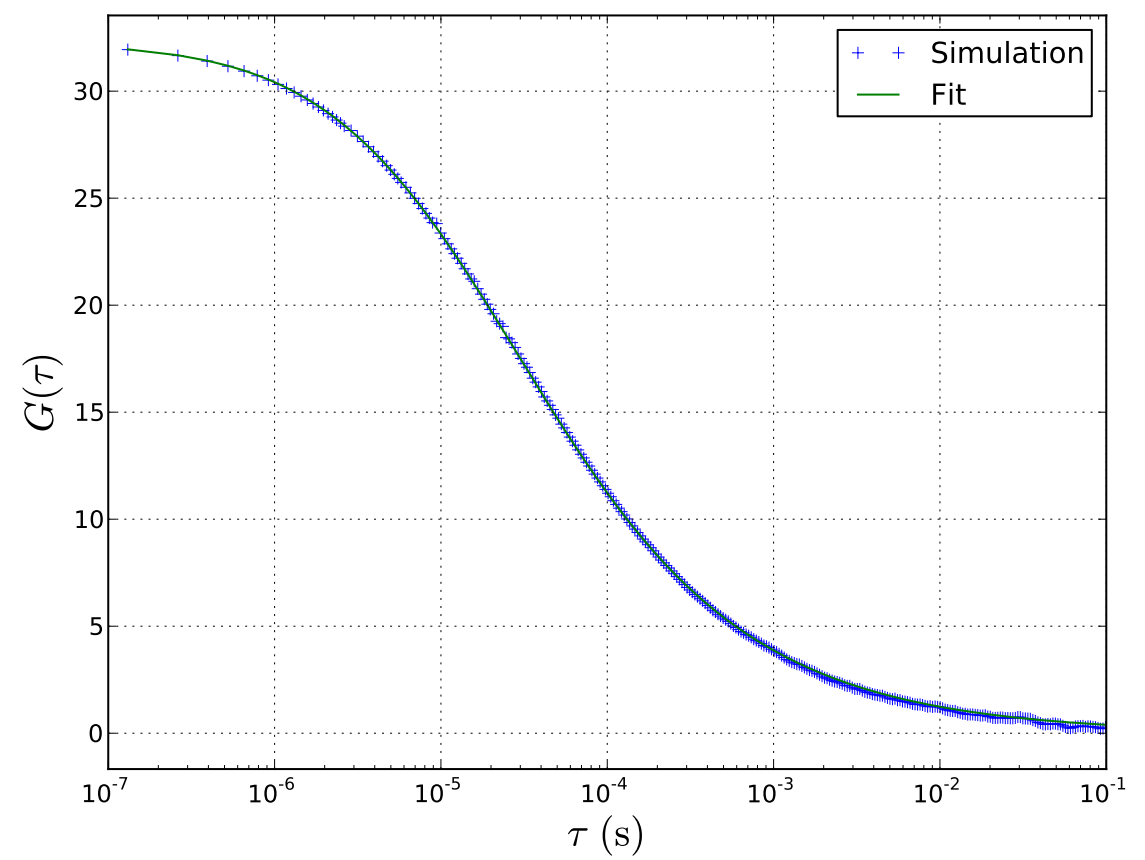

Figure 4.8.: Example of an FCS simulation in the case of total internal reflection with a decay length $d=70 \mathrm{~nm}$. The theoretical curve is given by equation 4.9. The simulation volume was $0.35 \times 0.35 \times 100 \mu \mathrm{m}^{3}$. The section of this volume in the $(x, y)$ plane can be chosen arbitrarily small as no artifical correlation is produced due to the reflection at the boundaries of the simulation volume because of the homogeneous intensity distribution in this plane. 20 molecules populate this volume, giving a concentration of about $0.34 \mathrm{nM}$. Photophysical and dynamical parameters of Atto655 were used for this simulation of $100 \mathrm{~s}$ with a time resolution of $100 \mathrm{~ns}$. 
correlation function is known. In the next section, the model is applied to near-field aperture probe in two cases, for which there are no analytical representations of the correlation function. From the comparison with known fit function, valuable insight is gained on the description of the intensity distribution at the near-field aperture probe.

\subsection{Modeling of the triangular aperture probe}

The intensity distribution at a near-field aperture probe was derived analytically only in the ideal case of a circular aperture in the infinitely thin and perfectly conducting material: this is the Bethe-Bouwkamp model and the corresponding FCS experiment with this kind of model is the object of the following section (4.2.1). However this intensity distribution cannot be expressed analytically in the case of a real near-field aperture and consequently it is not possible to obtain an analytical function describing the autocorrelation of the fluorescence signal of molecules diffusing at the aperture. Therefore a model of the actual aperture probe was implemented to get a map of the intensity distribution. The next section (section 4.2.2) deals with the details of this model and the results of the intensity calculations. Using the intensity distribution at the aperture, FCS simulations can be carried out and the main features of the corresponding autocorrelation are presented in the last section of this chapter (section 4.2.3).

\subsubsection{The Bethe-Bouwkamp model}

The only analytical solution that describes light interaction with an aperture much smaller than the wavelength of light is given by the Bethe-Bouwkamp theory [75], [76]. This theory applies to a circular aperture of radius $a$ in an infinitely thin and perfectly conducting screen. Bethe first stressed, that light diffraction at such small holes cannot be accurately described by the scalar formulation of Kirchhoff theory (see for example [77]) since boundary conditions at the aperture behind the screen (i.e. on the other side of the screen that is illuminated) are violated.

In order to satisfy these boundary conditions, Bethe's original idea consisted in deriving the diffracted fields from a fictitious magnetic charge density $\eta$ and a magnetic current density $\mathbf{K}$, from which the fields $\mathbf{E}$ and $\mathbf{H}$ can be derived using [76]:

$$
\mathbf{E}=\nabla \times \mathbf{F}, \quad \mathbf{H}=-\mathrm{i} k \mathbf{F}-\nabla \Psi
$$

where $\mathbf{F}$ and $\Psi$ are the magnetic vector potential and magnetic scalar potential and can be calculated as a function of $\mathbf{K}$ and $\eta$. Bouwkamp showed, however, that Bethe's analytical solution (first order approximation) is in error for K. As a result, a boundary condition in the hole is violated, which invalidates the fields $\mathbf{E}$ in and near the hole, whereas the fields $\mathbf{H}$ remains correct. The correct solution for the 
fields in and near the aperture $\left(r=\sqrt{x^{2}+y^{2}+z^{2}}<<a\right)$ for exciting fields of time harmonic dependence and amplitude $E_{0}, H_{0}$ is therefore [76], [29]:

$$
\begin{aligned}
& \frac{E_{x}}{E_{0}}=\mathrm{i} k z-\frac{2}{\pi} \mathrm{i} k a u\left\{1+v \operatorname{atan} v+\frac{1}{3} \frac{1}{u^{2}+v^{2}}+\frac{x^{2}-y^{2}}{3 a^{2}\left(u^{2}+v^{2}\right)\left(1+v^{2}\right)^{2}}\right\}(4) \\
& \frac{E_{y}}{E_{0}}=-\frac{4 \mathrm{i} k x y u}{3 \pi a\left(u^{2}+v^{2}\right)\left(1+v^{2}\right)^{2}} \\
& \frac{E_{z}}{E_{0}}=-\frac{4 \mathrm{i} k x v}{3 \pi\left(u^{2}+v^{2}\right)\left(1+v^{2}\right)} \\
& \frac{H_{x}}{H_{0}}=-\frac{4 x y v}{\pi a^{2}\left(u^{2}+v^{2}\right)\left(1+v^{2}\right)^{2}} \\
& \frac{H_{y}}{H_{0}}=1-\frac{2}{\pi}\left\{\operatorname{atan} v+\frac{v}{u^{2}+v^{2}}+\frac{v\left(x^{2}-y^{2}\right)}{\pi a^{2}\left(u^{2}+v^{2}\right)\left(1+v^{2}\right)^{2}}\right\} \\
& \frac{H_{z}}{H_{0}}=-\frac{4 a y u}{\pi a^{2}\left(u^{2}+v^{2}\right)\left(1+v^{2}\right)}
\end{aligned}
$$

where the oblate-spheroidal coordinate system was used $(\mathbf{r}=(u, v, \phi))$ and is related to the cartesian coordinate system by:

$$
\begin{aligned}
& x=a \sqrt{\left(1-u^{2}\right)\left(1+v^{2}\right)} \cos \phi \\
& y=a \sqrt{\left(1-u^{2}\right)\left(1+v^{2}\right)} \sin \phi \\
& z=a u v
\end{aligned}
$$

where $0 \leq u \leq 1,-\infty \leq v \leq \infty$ and $0 \leq \phi \leq 2 \pi$. Therefore, the surface $v=0$ $\left(x^{2}+y^{2} \leq a^{2}\right)$ corresponds to the aperture and the surface $u=0\left(x^{2}+y^{2} \geq a^{2}\right)$ to the screen.

These fields were implemented in the FCS simulation in order to compare the correlation curves with those resulting from different intensity distributions. Since the radial electric (derived from $E_{x}$ and $E_{y}$ with polar coordinates) and the normal magnetic $H_{z}(r=a)$ fields in the aperture at the edge diverge, a minimum distance between the aperture plane and the simulation volume has to be introduced. An FCS simulation example is shown in figure 4.9, where the aperture radius and the minimum distance to the aperture plane was set to be $30 \mathrm{~nm}$ and $1 \mathrm{~nm}$, respectively.

It is interesting to see that the correlation function of the FCS simulation of figure 4.9 can be fitted very well by a simple fit function that combines the analytical correlation function in two known cases. First for the fluorescence signal from molecules diffusing at an interface where light is totally internally reflected (see equation 4.9) and second, for the case where molecules diffuse in a two dimensional gaussian field (the second factor in equation 3.53 dependent on the intensity distribution in the $(x, y)$ plane). The corresponding function then writes:

$$
G(\tau)=\frac{1}{N}\left\{\left(1-2 \frac{D}{d^{2}} \tau\right) w\left[i\left(\frac{D}{d^{2}} \tau\right)^{\frac{1}{2}}\right]+2\left(\frac{\frac{D}{d^{2}} \tau}{\pi}\right)^{\frac{1}{2}}\right\}\left(1+\frac{4 D \tau}{R_{x y}^{2}}\right)^{-1}
$$




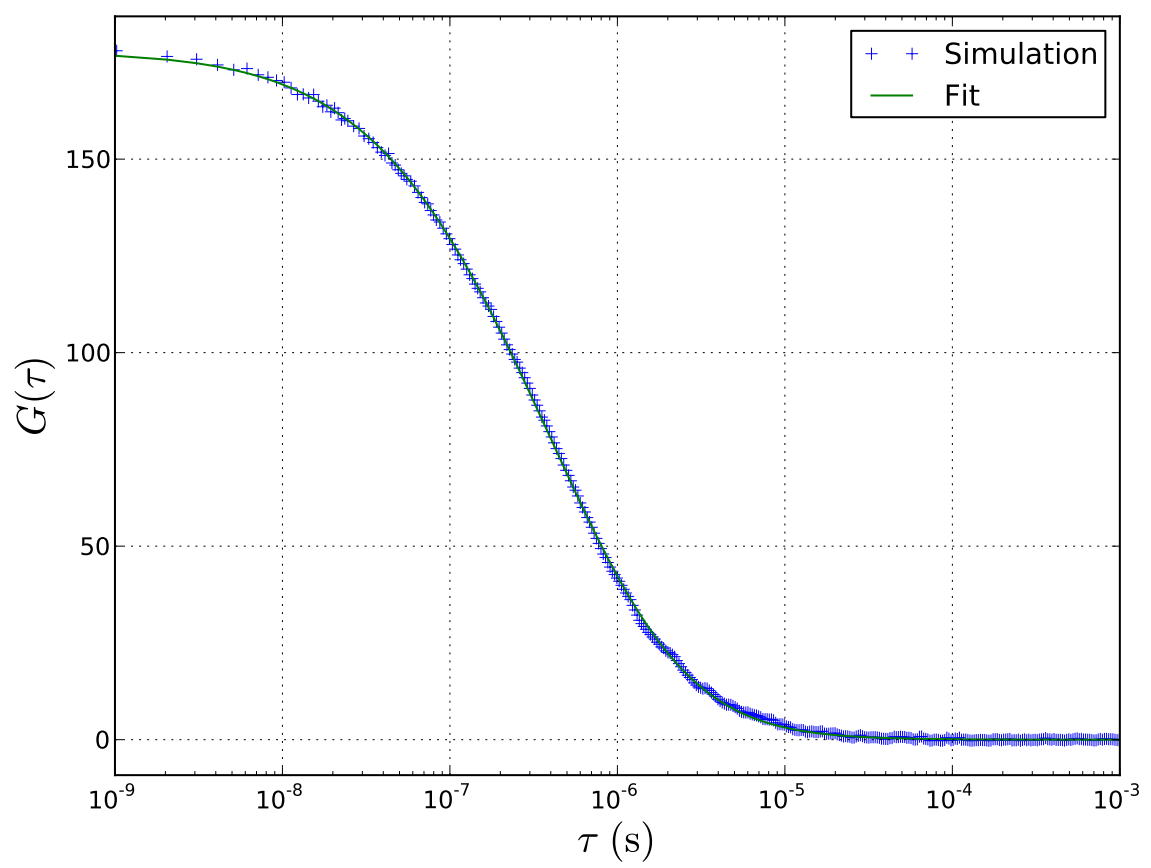

Figure 4.9.: FCS simulation example at an aperture of radius $30 \mathrm{~nm}$ with a minimum distance of the aperture plane to the simulation volume of $1 \mathrm{~nm}$. An exponential decay quenching model was used with a decay to $50 \%$ $5 \mathrm{~nm}$ away from the aperture and a decay from $90 \%$ to $10 \%$ within $3 \mathrm{~nm}$. The photophysical properties for Atto655 at $640 \mathrm{~nm}$ (excitation wavelength) were used (see section 4.1.2, TIRF modeling). The simulation was done with $1 \mathrm{~ns}$ resolution during $1 \mathrm{~s}$ and in a volume of $900 \times 900 \times 450 \mathrm{~nm}^{3}$. The function used to fit the simulation data is given in equation 4.22 . 
The choice of this function to model the data is not based on any derivation of the correlation function in the case of an intensity distribution given by the BetheBouwkamp model. This is a purely heuristic choice driven by the observation that a few nanometers away from the aperture, the intensity distribution turns into a Gaussian like distribution in the lateral plane.

The fit using this model is shown in figure 4.9 . Setting $D=426 \mu \mathrm{m}^{2} / \mathrm{s}$ correspondingly to the simulation provide a value of $8.68 \pm 0.04 \mathrm{~nm}$ for the decay length $d$ and $48.2 \pm 0.2 \mathrm{~nm}$ for the parameter $R_{x y}$. A surprisingly good fit is obtained with only three free parameters: $N, d$ and $R_{x y}$. This shows that, although the field distribution at the idealized aperture of the Bethe-Bouwkamp model is quite complex, the FCS signal is fairly equivalent to a simple exponential decaying field in the $z$ direction and a Gaussian profiled intensity distribution in the $(x, y)$ plane where $d$ and $R_{x y}$ are averaged value over the whole distribution. In the case of the simulation example given in figure 4.9 for an aperture radius of $30 \mathrm{~nm}$, the value $R_{x y}$ (intensity decay at $1 / e^{2}$ of the maximum intensity in the $(x, y)$ plane) makes sense since the confinement of light at the aperture is expected to decrease with increasing distance to the aperture. The value $d$ of about $10 \mathrm{~nm}$ (intensity decay at $1 / e$ of the maximum intensity along the $z$ axis) reflects the high axial confinement that is expected to be obtained at small apertures.

\subsubsection{Light intensity distribution at a less idealized aperture}

The model used to describe the aperture probe is a simplified model: it consists of a equilateral triangle of side length $80 \mathrm{~nm}$ being extruded in a gold film of $300 \mathrm{~nm}$ thickness (see figure 4.10). The extrusion process points out, that the pyramidal form of the real aperture (see section 5.1.1) is not taken into account at all in this model. However this model accounts for the finite conductivity of the metal and the ohmic losses inside it. This model of aperture is therefore, still idealized but more accurate than the Bethe-Bouwkamp model of the precedent section. The calculations of the intensity distribution were kindly carried out by Tolga Ergin using a finite element method implemented in the software package COMSOL Multiphysics of COMSOL.

The triangular aperture is illuminated at one side by a three dimensional Gaussian profile defined in cylindrical coordinates $\left(r=\sqrt{x^{2}+y^{2}}\right.$ and $\left.z\right)$ as follows:

$$
\mathbf{E}(r, z)=E_{0} \mathbf{u}_{x} \frac{w_{0}}{w(z)} \mathrm{e}^{-\frac{r^{2}}{w(z)^{2}}} \mathrm{e}^{\mathrm{i}\left\{k_{0}\left(z-\frac{r^{2}}{2 R(z)}\right)+\eta(z)\right\}}
$$

where $w_{0}=300 \mathrm{~nm}$ is the beam waist, $k_{0}=2 \pi / \lambda_{0}$ with the vacuum wavelength $\lambda_{0}=633 \mathrm{~nm}, w(z)=w 0 \sqrt{1+\left(z / z_{r}\right)^{2}}, R(z)=z\left(1+\left(z_{r} / z\right)^{2}\right), \eta(z)=\operatorname{atan} z / z_{r}$ and $z_{r}=\pi w_{0}^{2} / \lambda_{0}$. The electric field is polarized along the $x$ axis so that it is perpendicular to one of the triangular sides ( $\mathbf{u}_{x}$ is the unit vector along the $x$ axis) and its maximum amplitude is $E_{0}=1 \mathrm{~V} / \mathrm{m}$. The gold film is set to have a dielectric constant of $\epsilon_{\mathrm{Au}}=-10+\mathrm{i} 1.25$ where the imaginary part accounts for ohmic losses in the metal film. At the center of the metal coating the triangular aperture is 


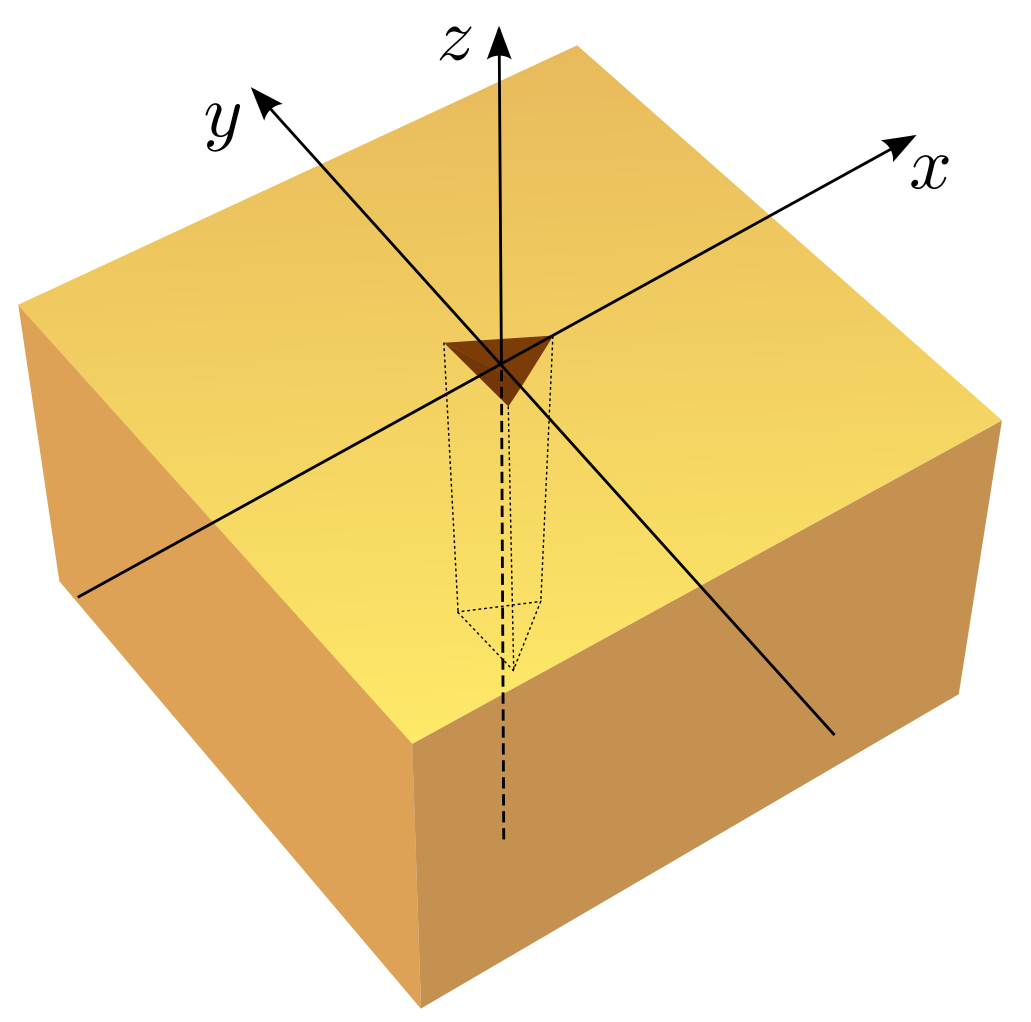

Figure 4.10.: The design of the aperture in a gold film used as a model of the nearfield aperture probe and used for the numerical calculation of the intensity distribution for the modeling of FCS with a near-field aperture probe. In the $(x, y)$-plane, the gold film is a square of $1000 \mathrm{~nm}$ side length. The height of the gold film is $300 \mathrm{~nm}$. The triangular aperture is equilateral and has a side length of $80 \mathrm{~nm}$. 
made of glass with the dielectric constant $\epsilon_{g}=2.25$. Notice that in equation 4.23, $z=0$ does not exactly correspond to $z=0$ in figure 4.10 but to $z=-300 \mathrm{~nm}$, i.e. not the plane separating water and gold but that separating vacuum and gold.

These numerical simulations delivered the different components of the electric $E_{i}$ and magnetic fields $H_{i}(i=\{x, y, z\})$ in a $1000 \times 1000 \times 380 \mathrm{~nm}^{3}$ large volume of water with a dielectric constant of $\epsilon_{\mathrm{H}_{2} \mathrm{O}}=1.7689$ directly under the aperture. However, for the simulation of the correlation function of the fluorescence signal only the electric fields are used to excite the fluorescence molecules according to the dipole approximation. Furthermore, the fluorescent molecules are supposed to rotate so fast on the scale of the smallest correlation time calculated for the correlation function, that the fluorescence signal is proportional to the time averaged of the squared amplitude of the electric field instead of its instantaneous value and orientation. As a result of losses in the metal coating, the electric fields contain an imaginary part $\Im\left(E_{i}\right)$ that must be taken into account for intensity calculations. This intensity is calculated as follows [77]:

$$
I=\frac{1}{4} \epsilon_{0} n_{W} \mathbf{E E}^{*}
$$

where $\epsilon_{0} \sim 8.85 \cdot 10^{-12} \mathrm{~F} / \mathrm{m}$ is the electric permittivity of vacuum and $n_{W}=1.33$ the refraction index of water. $\mathbf{E}$ represents the complex electric field vector with components $E_{i}=\Re\left(E_{i}\right)+\mathrm{i} \Im\left(E_{i}\right)$ with $i=\{x, y, z\}$. Instead of setting the excitation intensity before the aperture and consequently excite with the intensity calculated behind the aperture, the excitation intensity in the FCS simulation was set to be the maximum value of the intensity behind the aperture. The intensity map under the probe is consequently represented normalized to its maximum value in figure 4.11 in the plane of the aperture at the interface between gold and water $(z=0 \mathrm{~nm})$.

This numerical simulation is consistent with another simulation based on the field-susceptibility method [78] [39]. The main feature of the intensity directly under the aperture in the aperture plane is the confinement of the intensity at the rim to which the polarization is perpendicular. Additionally in this plane, this confinement can also be observed to be slightly under the glass side of the rim and have two maxima at its two corners (see figure 4.12, up). However, these last two features gradually vanish with increasing distance to the aperture. This can be observed at a distance of $z=30 \mathrm{~nm}$ in figure 4.12 (bottom).

Intensity profiles in the $x$ and $y$ direction at different distances $z$ to the aperture (see figure 4.13) show also that the intensity is more confined in the polarization direction. Additionally, the double maximum structure visible at $z=0 \mathrm{~nm}$ along the $y$ direction has vanished away after $z \sim 10 \mathrm{~nm}$. Over this distance, the intensity adopts an approximate Gaussian profile with different full width at half maximum (FWHM). Finally, the relative amplitude between the profiles at different distances $z$ from the aperture show the rapid decay of the intensity in this direction. Intensity profiles in this direction are shown in figure 4.14 for more clarity. The different curves correspond to different positions of the axis along which the intensity is evaluated and are characterized through the intersection of this axis with the aperture 


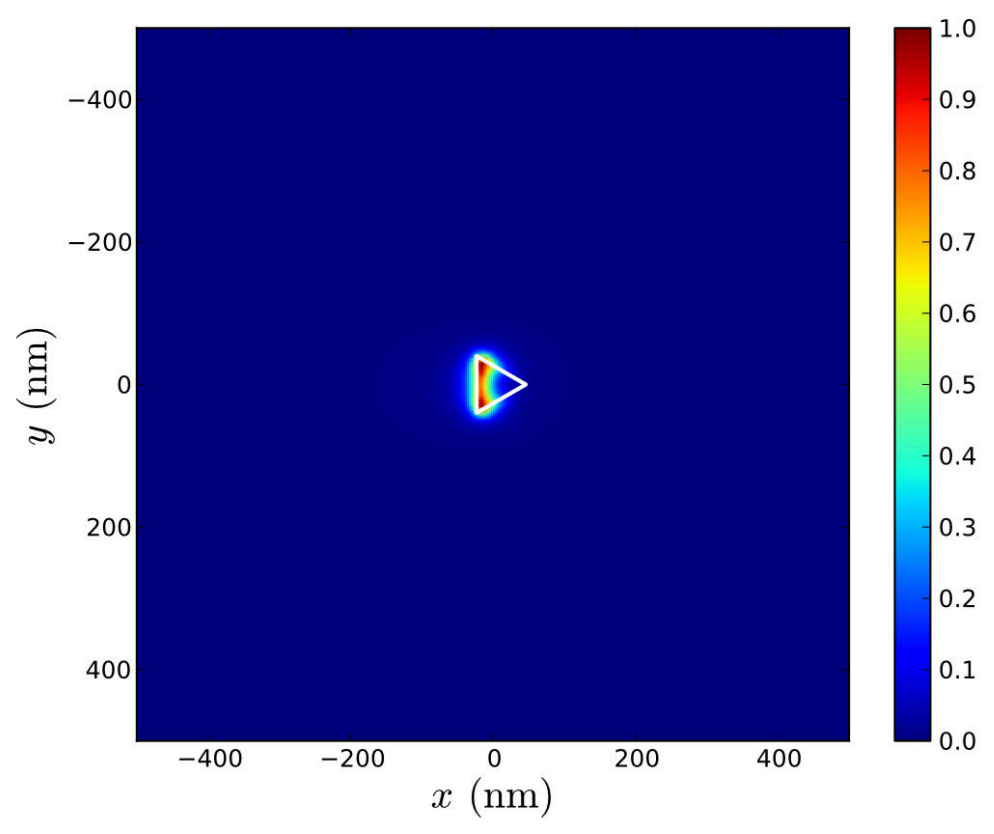

Figure 4.11.: Intensity map at the aperture probe model in the plane of the aperture at the interface between gold coating and water $(z=0 \mathrm{~nm})$. The white equilateral triangle represents the glass aperture with its $80 \mathrm{~nm}$ side length. The center of this triangle is located at $x=0 \mathrm{~nm}$ and $y=0 \mathrm{~nm}$. 

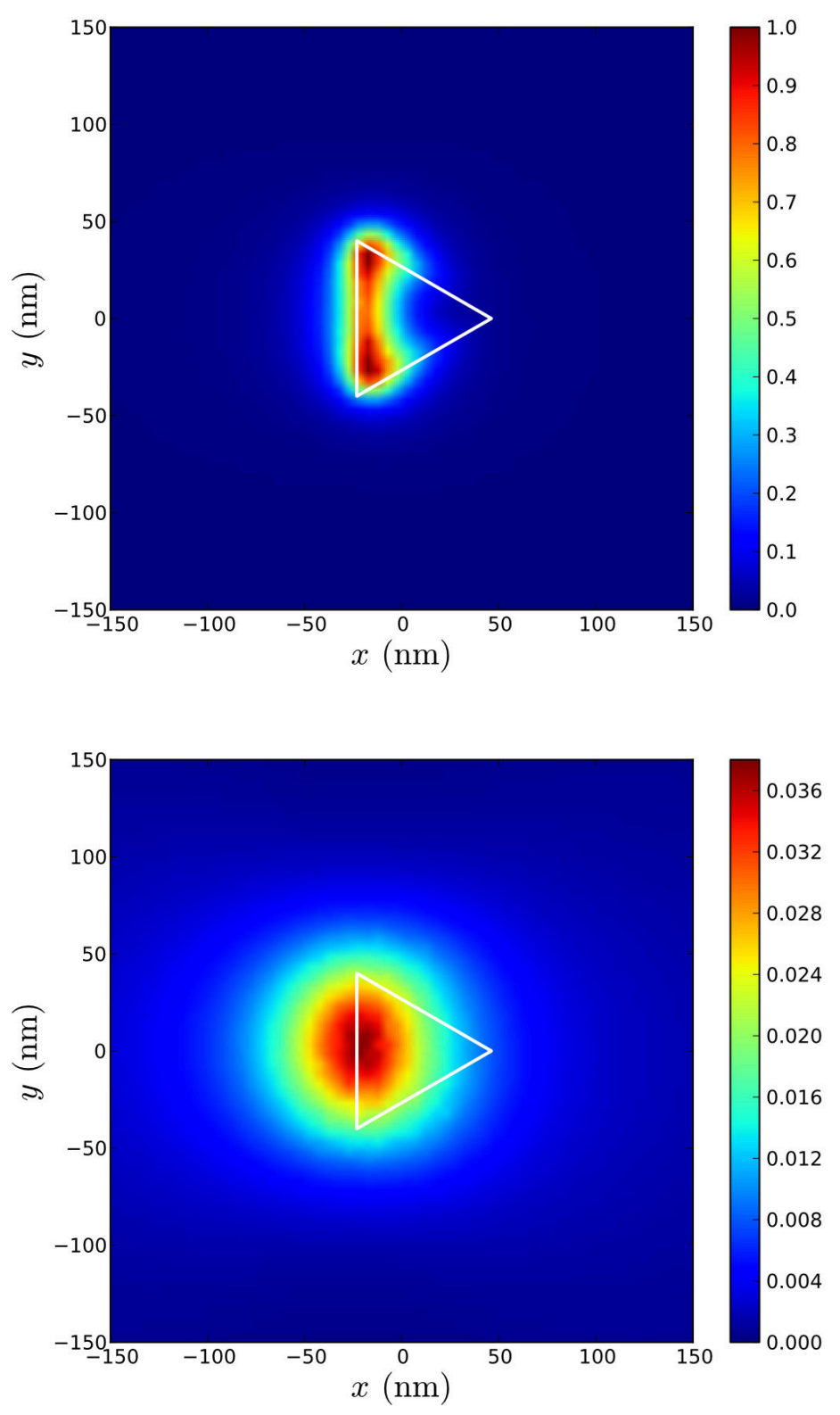

Figure 4.12.: Detailed view of the intensity map at two different distances from the probe aperture. Up: $z=0 \mathrm{~nm}$. Bottom: $z=30 \mathrm{~nm}$. With excitation light polarized along the $x$ axis, the intensity under the aperture is concentrated at the rim to which the polarization is perpendicular and on the glass side. Directly under the aperture a double maximum structure is also discernable that, however, very fast evolved in a single maximum located at the center of the rim, as the intensity is observed in planes perpendicular to the aperture axis with increasing distance to the aperture. Notice also the very fast decay of the intensity with $z$ by comparing the color bars. 
plane. From the three profiles, the one along the aperture axis $(x=0, y=0)$ has the smallest decay length whereas the profile intersepting one of the global intensity maxima $(x=-20, y=30)$ features a very rapid decay.

These different intensity maps and profiles show the high inhomogeneity of the electric fields under the aperture. However, the corresponding correlation function can be described in a relatively simple way as shown in the next section.

\subsubsection{FCS simulation at the triangular aperture probe}

In a similar manner to the idealized case of a circular aperture in a perfectly and infinitely thin conductor, FCS simulations were carried out using the simulated intensity distribution of the precedent section. An example of this kind of simulation is given in figure 4.15. For this simulation, 10 molecules with the photophysical and dynamical properties of Atto655 were enclosed in a $1000 \times 1000 \times 400 \mathrm{~nm}^{3}$ large volume and led to diffuse within 10 seconds. Because the intensity map was calculated with a resolution of $5 \mathrm{~nm}$, the time resolution $\tau_{\min }$ for the FCS simulation was set, so that the mean distance $l$ travelled during this time is on the order of the spatial resolution of the intensity map. Using $l=\sqrt{\left\langle x^{2}\right\rangle}=\sqrt{2 D \tau_{\min }}$ as guiding value, with $D=426 \mu \mathrm{m}^{2} / \mathrm{s}$, the time resolution was set to $10 \mathrm{~ns}$

Fitting the FCS simulation with equation 4.22 gives the values $N=(6.41 \pm 0.01)$. $10^{-2}, d=7.50 \pm 0.04 \mathrm{~nm}$ and $R_{x y}=115 \pm 1 \mathrm{~nm}$. A slightly better fit can be found by using th following equation:

$G(\tau)=\frac{1}{N}\left\{\left(1-2 \frac{D}{d^{2}} \tau\right) w\left[i\left(\frac{D}{d^{2}} \tau\right)^{\frac{1}{2}}\right]+2\left(\frac{\frac{D}{d^{2}} \tau}{\pi}\right)^{\frac{1}{2}}\right\}\left(1+\frac{4 D \tau}{R_{x}^{2}}\right)^{-1 / 2}\left(1+\frac{4 D \tau}{R_{y}^{2}}\right)^{-1 / 2}$

This equation is also a heuristic choice like equation 4.22 based on the observation that the intensity profiles in the plane $(x, y)$ are asymmetric (see figure 4.13 ). In the $x$ direction, which is the polarization direction, the intensity is better confined than in the $y$ direction. Using this equation to fit the FCS simulation of figure 4.15 gives $N=(6.45 \pm 0.02) \cdot 10^{-2}, d=7.73 \pm 0.06 \mathrm{~nm}, R_{x}=77 \pm 2 \mathrm{~nm}$ and $R_{y}=230 \pm 20 \mathrm{~nm}$. The standard deviation of the fit is also improved by about $5 \%$. The accuracy of the fit renders well this asymmetry in the intensity distribution and shows that in the polarization direction, the intensity is nearly as confined as for the idealized model of the circular aperture. Given the fact that a finite penetration depth of the excitation light is totally neglected in the Bethe-Bouwkamp model, this result shows the quality of light confinement at a triangular aperture. The axial light confinement seems to be higher for the triangular aperture than for the circular aperture in the Bethe-Bouwkamp model. This could be due to the fact that the circular aperture has two maxima in the intensity distribution at the edges of the aperture intersecting the polarization direction, whereas the triangular aperture has only one in this direction. As the distance $z$ to the aperture probe increases, 

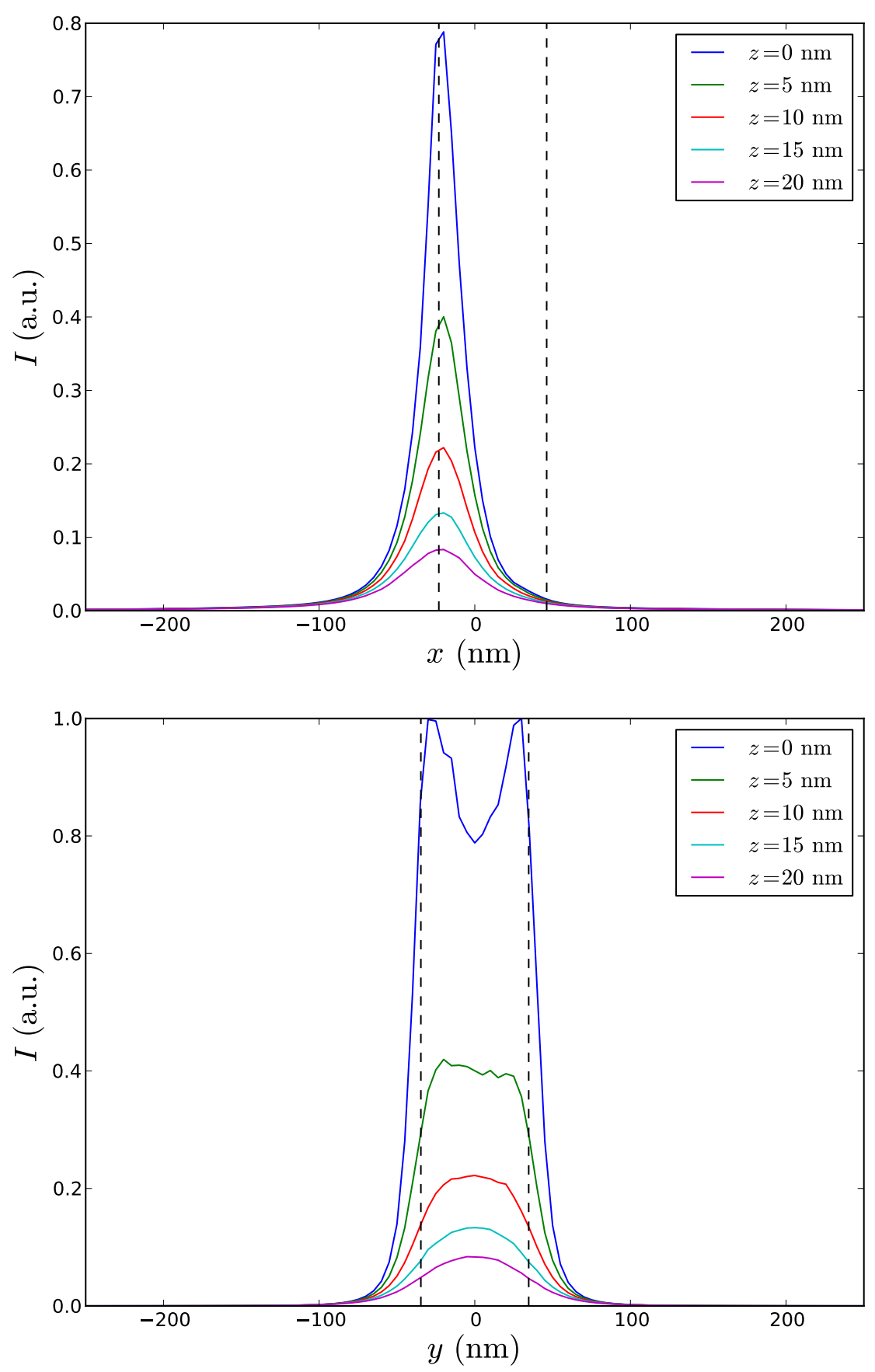

Figure 4.13.: Light intensity under the aperture along different axis and at different distances from the aperture probe. Above: along the $x$ axis at position $y=0 \mathrm{~nm}$ (i.e. the axis getting through the triangle center at $x=$ $0 \mathrm{~nm}$ ). Below: along the $y$ axis at position $x=-20 \mathrm{~nm}$ (i.e. the axis getting through the double maximum structure from figure 4.12 at $z=0 \mathrm{~nm}$ ). Above about $10 \mathrm{~nm}$ distance from the aperture the intensity profiles resemble Gaussian profiles. The black dotted lines represent the position of the rims. 


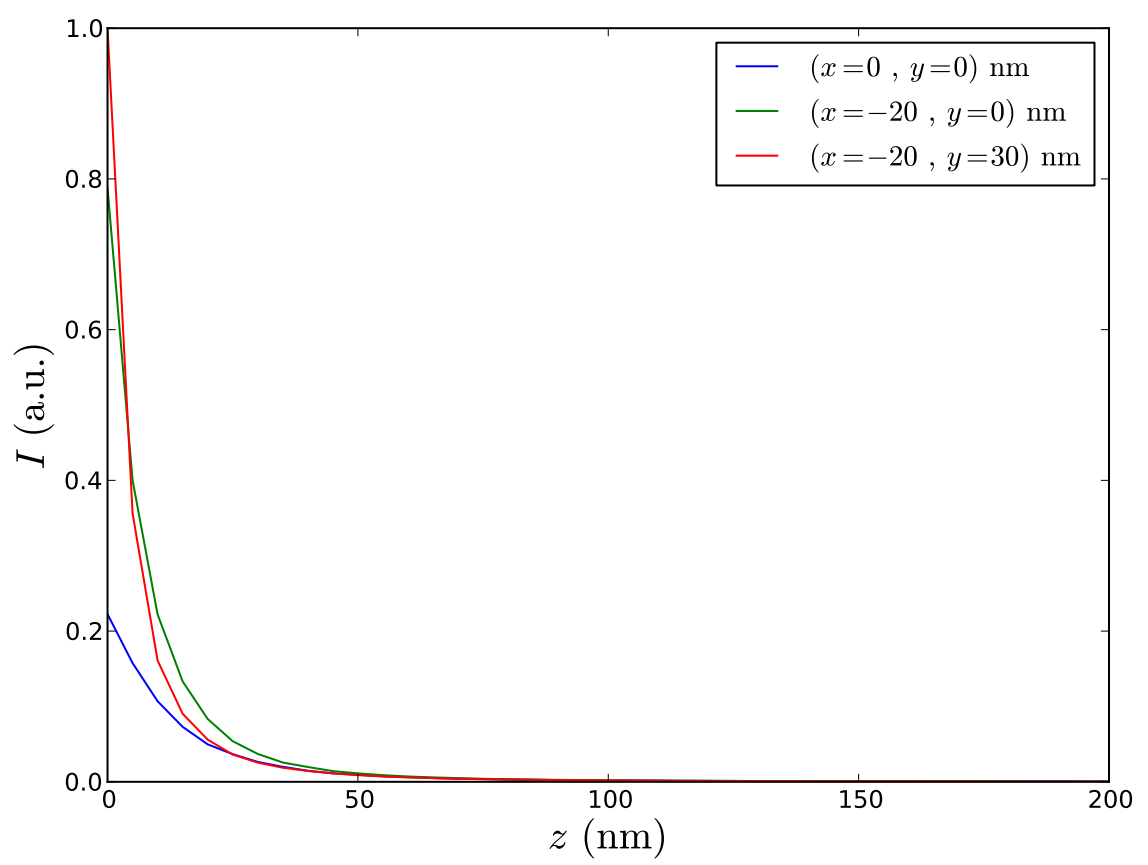

Figure 4.14.: Light intensity along a direction perpendicular to the aperture plane for different positions $(x, y)$ in the aperture plane. $(x=0, y=0)$ corresponds to the center of the triangle and the corresponding intensity represents therefore the intensity along the symmetry axis of the triangle. $(x=-20, y=0)$ corresponds nearly to the middle of the rim, to which the polarization of the excitation light is perpendicular. $(x=-20, y=30)$ corresponds to the position in the aperture plane of the intensity maximum. It can be seen that the intensity decay with increasing distance to the aperture is very fast and corresponds approximately to an exponential decay. However, the decay length of these intensity profiles is also dependent on the position $(x, y)$ at the intersection of the axis, along which the profile is considered, with the aperture plane. 


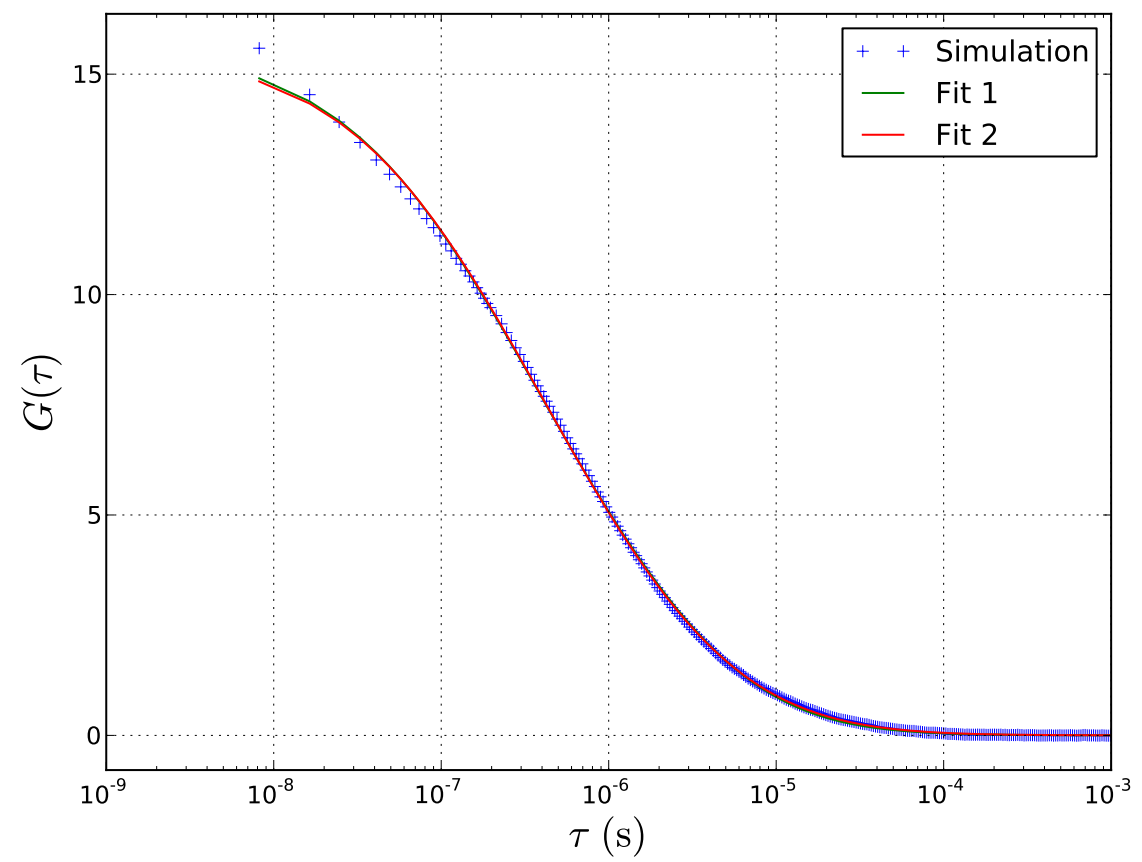

Figure 4.15.: FCS simulation example using the intensity distribution model calculated in section 4.2.2. The side length of the triangular aperture is $80 \mathrm{~nm}$ and the light polarization is set perpendicular to one rim of the triangle. The simulation volume is $1000 \times 1000 \times 400 \mathrm{~nm}^{3}$ populated with 10 Atto655 molecules (see parameters in table 4.1), which corresponds to a concentration of about $40 \mathrm{nM}$. The quenching model used is the same as in figure 4.9 and the intensity maximum was $1000 \mathrm{~kW} / \mathrm{cm}^{2}$ (excitation wavelength: $640 \mathrm{~nm}$ ). The simulation was carried out for one second with a resolution of 10 ns. Fitting was done either with equation 4.22 (Fit1) or equation 4.25 (Fit2). 
the contribution of the two maxima for the circular aperture leads to a slightly slower overall decrease of the intensity.

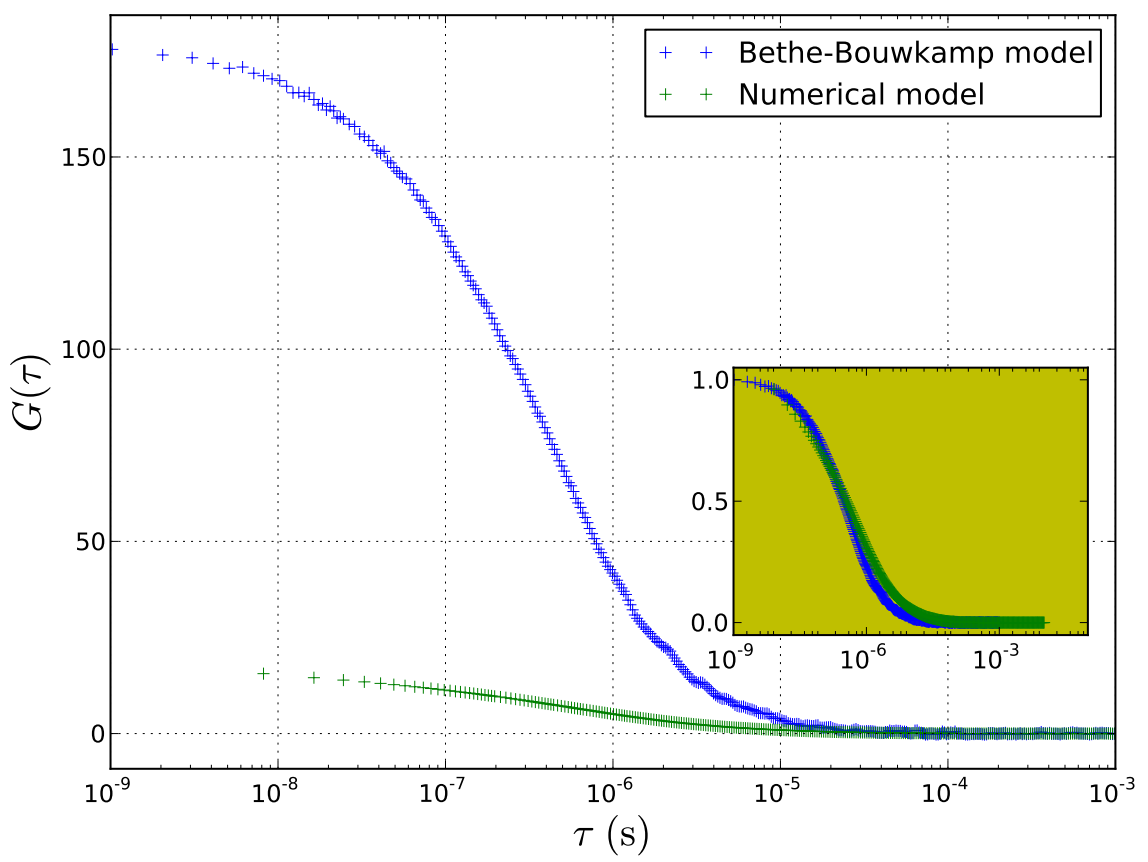

Figure 4.16.: Comparison between the two simulations of figure 4.9 and 4.15 showing FCS simulations with the idealized aperture with radius $30 \mathrm{~nm}$ of the Bethe-Bouwkamp model and with the triangular aperture with side length $80 \mathrm{~nm}$. These dimensions correspond to the same surface of the aperture. The other simulation parameters were kept the same. The inset shows the normalized correlation function.

Given that the amplitude of the correlation at zero lag time $G(0)$ for free diffusion only depends from the mean number of particles $\langle N\rangle$ in the excitation volume $V_{\text {exc }}$ (see section 3.2.2), its size can be calculated from the fit value of $N$ and the concentration used for the simulation. This concentration corresponds to 10 molecules in a $0.4 \mu \mathrm{m}^{3}$ simulation volume. It results in an excitation volume of about $3 \mu \mathrm{m}^{3}$ or 3 attoliters. It can be calculated straightforward, that using such an excitation volume, contains in average one molecule at a concentration of $0.5 \mu \mathrm{M}$. This simulation with the model aperture probe shows therefore that the triangular aperture is eligible for FCS measurements in the microcmolar range.

Using the size of the excitation volume found before, an expression can be given for $V_{\text {exc }}$ as a function of the parameters $d$ and $R_{x y}$ or $d, R_{x}$ and $R_{y}$ depending on the model used to describe the FCS curve. Similarly to the expression for the excitation volume created by a focused Gaussian beam, $V_{\text {exc, G }}=\pi^{3 / 2} R_{x y}^{2} R_{z}$, the 
excitation volume at the model triangular aperture probe can be expressed as:

$$
V_{\mathrm{exc}}=\left\{\begin{array}{r}
\alpha_{1} R_{x y}^{2} d \\
\alpha_{2} R_{x} R_{y} d
\end{array}\right.
$$

By introducing the fit values for $d$ and $R_{x y}$ and $d, R_{x}$ and $R_{y}$ and equating this volume with the 3 attoliters found before, the parameters $\alpha_{1}$ and $\alpha_{2}$ can be evaluated for the circular and elliptic intensity distributions in the lateral plane, respectively. The values $\alpha_{1} \approx 30.3$ and $\alpha_{2} \approx 21.4$, respectively, were found.

For the sake of completeness, the two FCS simulations from figure 4.9 and 4.15 , one with the idealized aperture of the Bethe-Bouwkamp model and one with the triangular aperture respectively, are compared in figure 4.16. The amplitude of the autocorrelation functions are inversely proportional to the mean number of molecules in the excitation volume (see equation 3.55). Due to the fact that the concentrations in the two simulations are the same, the excitation volumes can be compared. For the idealized aperture, the excitation volume is slightly more than one order of magnitude larger than that of the triangular aperture probe. This is not surprising since, for example, the skin depth does not play a role in the analytical model, whereas in the numerical model, this causes electromagnetic fields to leak through the metal coating, where it is thin enough, i.e. directly at the aperture. Consequently, the confinement is not as good, which explains the difference in the size of the excitation volumes. 


\section{Experimental realization of near-field optical fluorescence correlation spectroscopy}

This chapter deals with the experimental fabrication of a near-field optical probe at the aperture of which fluorescent molecules in a solution can diffuse. It also explains the setup enabling to measure the fluorescence signal stemming from these molecules and finally how the autocorrelation of this signal is calculated.

\subsection{Fabrication of the aperture probes and setup of the near-field optical microscope}

\subsubsection{Probe fabrication}

The near-field aperture probe is the heart of this study as it is the nanostructure that enhances the light intensity in a small region of space around the aperture, the near-field, i.e. at distances to the aperture much smaller than the wavelength. The fabrication process of this probe consists in different steps that are explained below.

\section{Glass cleaving and probe assembly}

The nanostructure designed to enhance electromagnetic radiations is actually the sharp end of a glass tip. Using the property of glass to create very sharp edges upon breaking, a tip is created by breaking a piece of glass in two perpendicular directions [39]. The resulting edge of the tip has a curvature radius of a few nanometers and can consequently be used as the basis for the nanostructure. Coating the structure with a metal of adequate thickness and uncapping it at a few nanometers of the end of the glass tip shall afterwards lead to the aperture probe [39].

First of all the glass pieces that will be used to form the probe are sonicated (Sonicator Sonorex Super RK100H, Bandelin GmbH \& Co. KG) in a 1\% Hellmanex II solution (Hellma $\mathrm{GmbH}$ ) for 15 minutes at $70^{\circ} \mathrm{C}$ and then rinsed with a copious amount of ultrapure water (resistivity greater than $18 \mathrm{M} \Omega \mathrm{cm}$ and provided from a Direct-Q 3 UV Water Purification System, Millipore). The glass pieces are small glass coverslips of $10 \times 10 \mathrm{~mm}^{2}$ surface and $0.3 \mathrm{~mm}$ thickness (Plano $\mathrm{GmbH}$ ), right angled glass prisms with a $4.5 \mathrm{~mm} \times 3.2 \mathrm{~mm}$ basis and a height of $1.6 \mathrm{~mm}$ (Optikron 
$\mathrm{GmbH}$ ) and $18 \mathrm{~mm} \times 18 \mathrm{~mm}$ coverslips with thickness \#1, which corresponds to a thickness of $145 \pm 15 \mu \mathrm{m}$ (Menzel GmbH). For the further fabrication process, it should be taken care of minimizing contamination by dust particles by, e.g., cleaning the working surface with isopropanol and acetone. These precautions together with the cleaning process ensure that no dust particle will stick at the end of the sharp tip before the metallization process as it can dramatically reduce the quality of the probe.

After the glass pieces dried (at ambient air or under a nitrogen flow), the glass tip is cut out of the thin coverslip (with thickness $145 \pm 15 \mu \mathrm{m}$ ) using a two step scratch-break process described in figure 5.1. The scratching was done using a diamant cutter (S90W, Thorlabs). At the end of this process a small triangle with the sharp tip as apex and with base length corresponding to the longest prism side $(4.5 \mathrm{~mm})$ is detached from the coverslip.

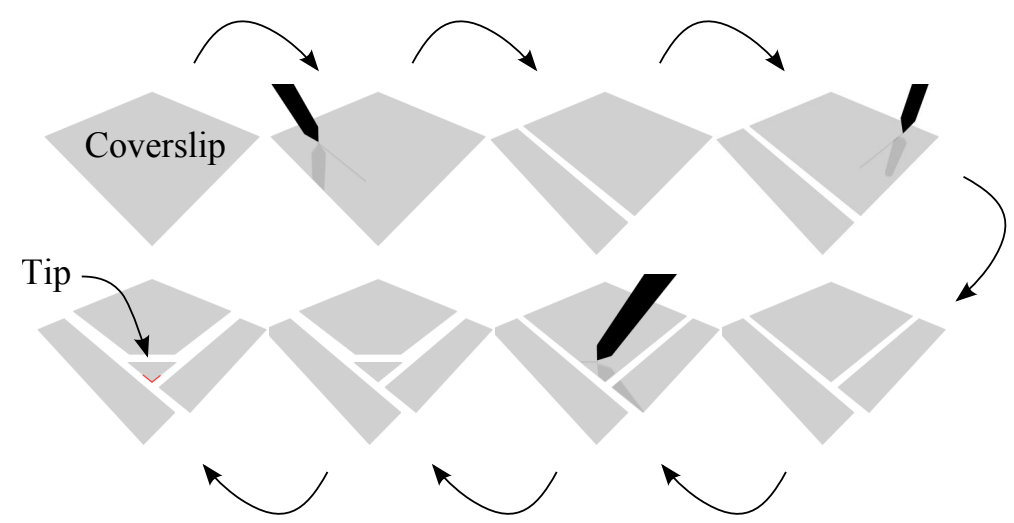

Figure 5.1.: The cleaving process of a $145 \pm 15 \mu \mathrm{m}$ thick $18 \mathrm{~mm} \times 18 \mathrm{~mm}$ coverslip is realized under the optical microscope (10× magnification). A diamant cutter is used to scratch the glass on a small part of the glass to facilitate breaking in a given direction. This process is repeated in the orthogonal direction so that a sharp tip is created. Finally a small triangle of basis $\sim 4.5 \mathrm{~mm}$ with the tip as apex (red mark in the last image) is detached from the glass coverslip for further processing.

Following this scratch-break process the different glass pieces are assembled using a glue (NOA81, Norland) with refractive index matching the one of glass and that hardens under ultraviolet exposure (ultraviolet lamp UVAHAND, Dr. Höhnle AG). Further working under the optical microscope $(10 \times$ magnification), the prism is first glued at the center of the thick coverslip $0.3 \mathrm{~mm}$. Then to facilitate further manipulation of the small glass triangle, the tip of a glass fiber is glued to the glass triangle approximately at the middle of the triangle. Finally, the glass triangle is glued at one of the free side of the glass prism lying on the thick coverslip. After the glue has completely dried, the glass fiber is moved away from the glass triangle by translation and rotation movements around the glue spot. This removal of the glass fiber without removing the glass triangle from the glass prism is possible, since 
the glue area between the fiber and the triangle is much smaller than the glue area between the glass triangle and the glass prism. These different steps are illustrated in figure 5.2.

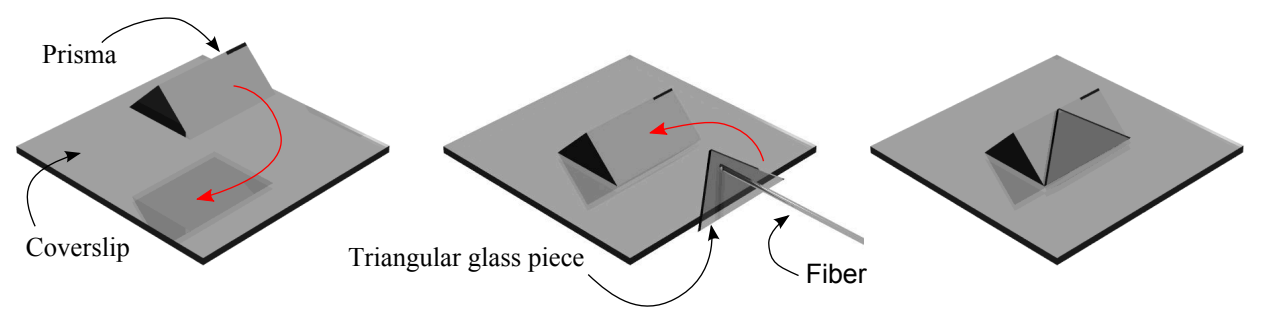

Figure 5.2.: To assemble the different glass pieces, the glue NOA81 (Norland) is used because it quickly cures under ultraviolet light exposure and its matched refractive index to glass after it has cured. First the glue is given in the middle of the coverslip and the prisma is laid on it as centered as possible (under the $10 \times$ magnification of a stereo microscope). Curing is performed under ultraviolet exposure by maintaining the prism in place with tweezers. Afterwards the glue is put on one side of the prism and the triangle glass piece obtained from the scratchbreak process is laid on the prism using a glass fiber. Ultraviolet light curing is carried out once more and the fiber is pulled away. The image on the right illustrates the probe before the coating process. For each assembly a minimal amount of glue is used so that it does not spread over the entire glass parts.

\section{Probe metallization}

Once the assembling is finished, the uncoated glass probe is put in a vacuum coater (Auto500, BOC Edwards) straight away, to avoid contamination with dust particles. The coating process is a very important step as it will directly influence the confinement of the light at the aperture. The coating is carried out by magnetron sputtering with a direct current (d.c.) power supply. In this kind of sputtering technology the metal to be coated (called the "target") is placed on an electrode at a negative voltage. After vacuum pumping, an inert gas (argon) called the "process gas" flows in the vacuum chamber. Through random processes such as thermal collisions, some of the argon atoms become ionized and are accelerated at the target. The energies transmitted to the target reach higher values than the binding energy of the metal atoms in the target so that these atoms are catapulted away from the target. At the target, the ions of the inert gas recombine with electrons. But the ejection of atoms out of the target comes along with the emission of secondary electrons. Some of these electrons are trapped in a magnetic field surrounding the target. These electrons can cause additional neutral gas atoms to ionize, which provokes a new bombardment of the target and the ejection of a target atom. This 
characteristic feature of magnetron sputtering enables to sputter at lower process gas pressure. At low pressure, the neutral atoms that are ejected from the target have a higher mean free path, since a collision with process gas atoms becomes less probable and a more directional coating can be achieved [79].

For the usual case where the metal to be coated is gold (gold target from Matek GmbH, purity: 99,99\%), the vacuum chamber is first pumped at a pressure of about 1 to $2 \cdot 10^{-6} \operatorname{mbar}\left(0.75\right.$ to $1.5 \cdot 10^{-6}$ Torr). This is already sufficient to achieve good coating results as gold is very inert and therefore does not tend to react with residual gases such as water vapor in the vacuum chamber. Once this pressure is reached an argon flow of $10,000 \mathrm{sccm}^{1}$ is set to get through the vacuum chamber. The probes lie about $13.5 \mathrm{~cm}$ away from the target on a holder that can contain up to 10 probes distributed on a circle of radius $2.5 \mathrm{~cm}$ and their tips are oriented in direction of the target. Additionally the long side of the prism is oriented in direction of the tangent at the point of the circle where the probe lies on the holder so that the triangular glass piece of the probe is on the outer side of this circle (see figure 5.3). The holder is also allowed to rotate about its own axis during the sputtering process to avoid possible asymmetry in the film coating due to a misalignment of holder axis and target axis.
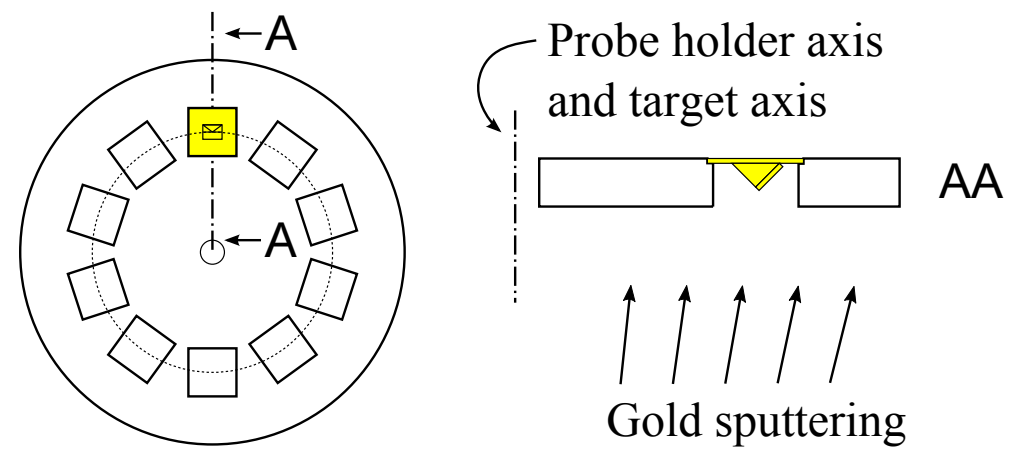

Figure 5.3.: Design of an aluminum holder for the metal coating of the probes. The axis of the holder corresponds to the axis of the target that is $13.5 \mathrm{~cm}$ away. The probes lie $25 \mathrm{~mm}$ away from the axis of the holder and are held so that the triangle glass piece obtained from the scratch-break process are on the outer part of the circle on which the probes are distributed (dashed line in the left image)

For FCS measurements in water and aqueous buffers, gold is usually the metal of choice to coat the near-field optical probes. Contrary to aluminum, it does not dissolve when exposed to the high temperature induced at the end of the tip. But gold also does not readily stick to a clean glass surface when sputtered. Therefore, an adhesive layer has to be coated on glass before the actual gold coating. In

\footnotetext{
${ }^{1}$ one standard cubic centimeter per minute represents one cubic centimeter per minute at the standard condition for temperature and pressure, especially the molar volume $V_{\text {mol }}$ of a gas in these conditions is $V_{\mathrm{mol}} \sim 22.4 \mathrm{~mol} / \mathrm{L}$
} 
this work, an adhesive layer made out of chromium was used although titanium is another common adhesive layer of choice [80]. Due to the large light absorption of chromium the adhesive layer must not be coated too thick otherwise the intensity at the end of the probe becomes too small. In order to evaluate the chrome thickness coated on the glass probe, such a probe was coated with chromium with a power supply of $100 \mathrm{~W}$ during 15 minutes. The film thickness was then evaluated by milling the aperture with a focused ion beam (see next step) and measuring the film thickness on an electron micrograph [81]. This resulted in a chromium sputter rate of about $0.35 \mathrm{~nm} / \mathrm{s}$. Consequently, the chromium sputtering was limited to $10^{\prime \prime}$, which results in a $3.5 \mathrm{~nm}$ thick chromium layer in the conditions enumerated above. After the glass probe is coated with a chromium layer, a gold film is sputtered during $3^{\prime} 30^{\prime \prime}$ with a power supply of $400 \mathrm{~W}$. In electron micrographs of the nearfield aperture probe (see figure 5.6) the chromium layer is not visible, which is compatible with a resolution of about $5 \mathrm{~nm}$ of the electron microscope and the desired thickness of $3.5 \mathrm{~nm}$. The gold film thickness reaches about $180 \mathrm{~nm}$. At this point the coated probe (see figure 5.4) reflects the entire incident light on the uncoated face of the probe.

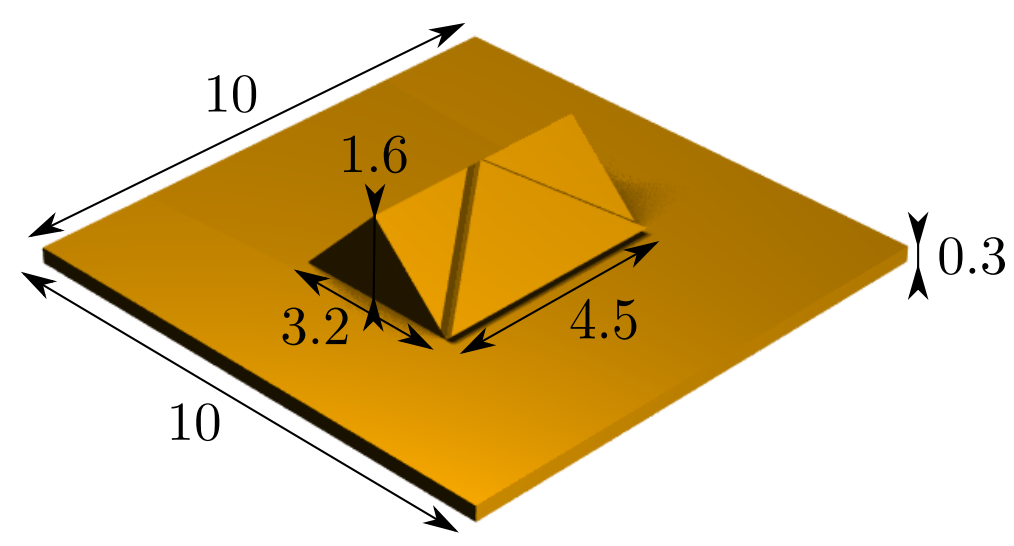

Figure 5.4.: Overview of the design of the near-field probe before the aperture creation process. All the dimensions on the image are given in millimeters. The basis is a glass coverslip of $10 \times 10 \mathrm{~mm}^{2}$ and with a thickness of $0.3 \mathrm{~mm}$. At its center lies a right angled glass prism with basis $4.5 \times 3.2 \mathrm{~mm}^{2}$ and height $1.6 \mathrm{~mm}$. A piece cut out of a glass coverslip of thickness $145 \pm 15 \mu \mathrm{m}$ so that its longest edge has also a dimension of $4.5 \mathrm{~mm}$, is glued on one of the widest sides of the prism. The sharp end of this glass piece is the near-field aperture tip and it lies at the centre of the edge of the prism so that the tip is the summit of the probe. Finally, a gold coating is applied on the glass structure from the side where the prism is glued on the large coverslip. The other side (the lower side of the coverslip in this image) is left uncoated. 


\section{FIB mlling}

To make a near-field aperture probe out of this coated probe a last step is needed: uncapping the metal coating at the very end of the pyramidal tip. This is achieved by a focused ion beam (FIB) instrument (CrossBeam 1540 XB, Carl Zeiss GmbH) from the Laboratory for Electron Microscopy at the Karlsruhe Institute of Technology and kindly operated by Patrice Brenner. In a FIB instrument, a small amount of gallium $(\mathrm{Ga})$ is vaporized upon heating, then ionized and finally accelerated towards the sample. The high momenta of these ions are used to bounce out atoms of a sample. The $\mathrm{Ga}^{+}$ions are focused and deflected with electrostatic lenses so that a FIB can be used to create nanostructures. In the case of a near-field optical probe, the Ga ion beam is scanned in the plane containing the tip and perpendicular to the base coverslip of the near-field probe with the fast axis being perpendicular to the tip axis. Starting at a secure distance away from the tip, the focused ion beam (ion beam current: $5 \mathrm{pA}$, ion energy: $30 \mathrm{keV}$ ) slowly approaches the tip and at some points atoms of the coating metals at the very end of the tip are removed. This milling procedure is carried on until the underlying glass is visible and is stopped when the glass aperture in the metal has reached the desired size, typically between 20 and $100 \mathrm{~nm}$ (see figure 5.5). The size of the aperture is controlled in the FIB instrument with an electron gun working as a scanning electron microscope (SEM) with an electron high tension (EHT) of $2 \mathrm{kV}$ (see detailled information in [81]).

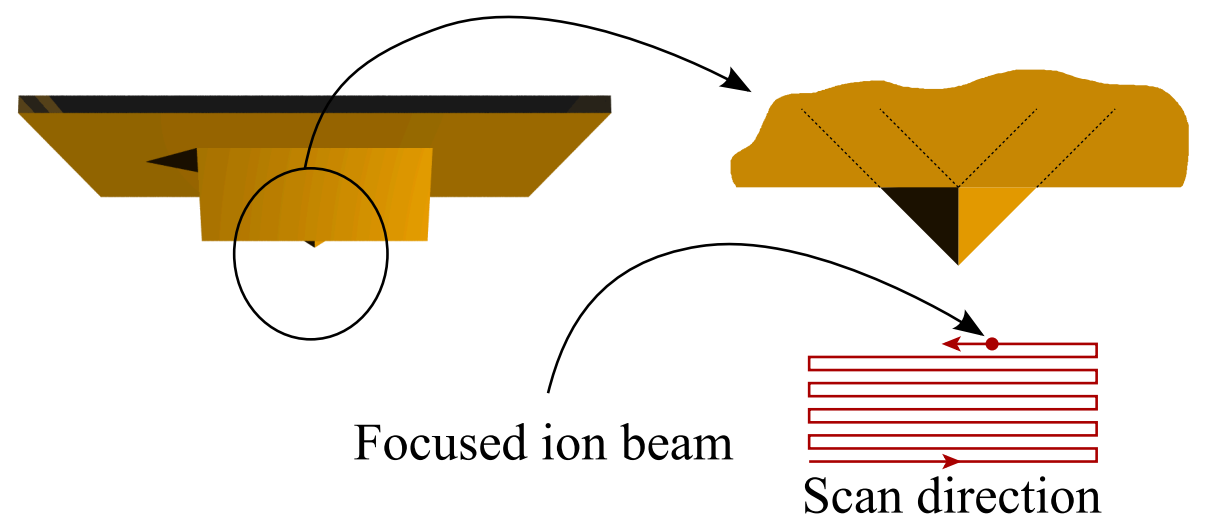

Figure 5.5.: To create an aperture in the metal coating of the probe, a focused gallium ion beam is slowly scanned under the aperture with an ion beam current of $5 \mathrm{pA}$ and an ion energy of $30 \mathrm{keV}$. The cutting plane is the plane perpendicular to the symmetry axis of the pyramidal tip of the triangular glass piece. As a result of the scanning of the beam, a triangular glass aperture is milled out of the metallic coating. The milling of the aperture is stopped as soon as the desired size of the glass aperture is reached (between 20 and $100 \mathrm{~nm}$ )

Two examplary images of the aperture milling process are shown in figure 5.6. The first one (left image) represents the ideal near-field optical probe with a symmetrical metal coating around the glass aperture. On the right image the asym- 
metric coating is due to a different orientation of the glass triangular piece lying on the prism of the near-field probe. This is a consequence of the very directional sputtering process and the triangular symmetry of the near-field optical probe.

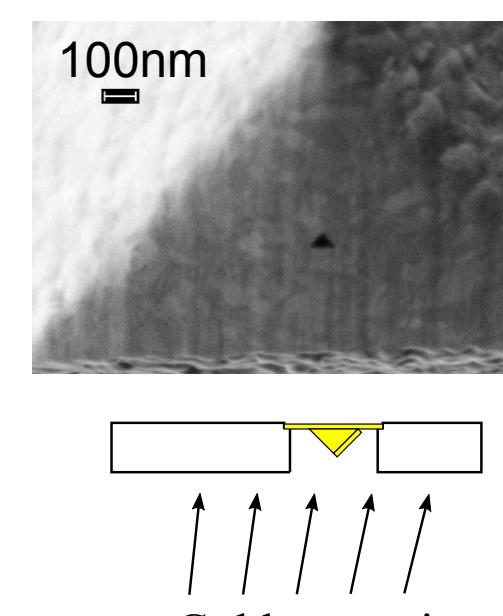

Gold sputtering

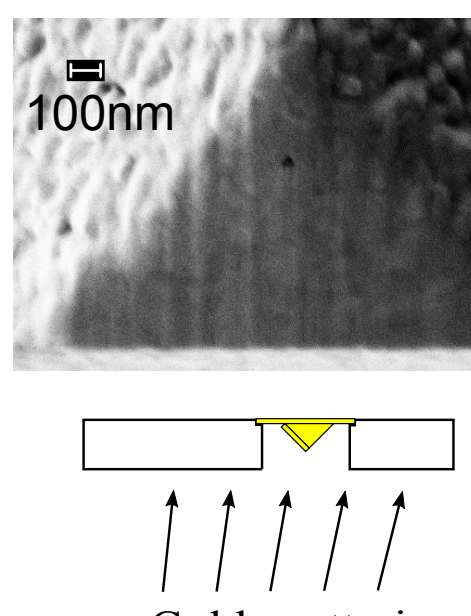

Gold sputtering

Figure 5.6.: Electron micrographs after uncapping the metal coating at the pyramidal tip. The triangular glass aperture is visible as a dark triangle surrounded by a brighter zone that represents gold. Depending on the orientation of the probe to the target in the vacuum coating device a symmetric or asymmetric coating can be observed (see figure 5.3). The vertical lines that can be observed on the flat end face of the probe are the result of scanning with the focused ion beam (see figure 5.5). The structure also visible at the flat end face with brighter and darker zone results from the different orientations of the metal grains, which causes a different emission of the secondary electrons that are responsible for the imaging process.

This overall fabrication process is highly reliable. Nearly $100 \%$ of the metallized probes display a well defined triangular aperture at the apex upon FIB milling considering their electron micrographs. Using batches of ten probes, different aperture sizes or, alternatively, many probes of a given aperture size can be fabricated with exactly the same sputtering conditions. This is an important aspect if the sputtering conditions vary from one batch to the other. However, focusing laser light in the probe and observing the diffraction pattern under the optical microscope with 20 fold magnification can reveal an asymmetric diffraction pattern. Such a pattern is usually caused by light leaking through small holes in the metal coating. In this, the probe is unusable for NSOM-FCS measurements. After inspection under the light microscope, above $50 \%$ of the probes fabricated following the present process can be used for NSOM-FCS. 


\subsubsection{Measurement setups}

\section{FCS measurements with a NSOM probe}

Excitation setup Once the near-field optical probe has been fabricated, coated and an aperture has been created with a FIB, it has to be properly illuminated with light in order to obtain an efficient coupling of light into the near-field of the probe aperture. To achieve this a dedicated setup called "excitation setup" was mounted and is explained in the following text. Figure 5.7 illustrates also this setup.

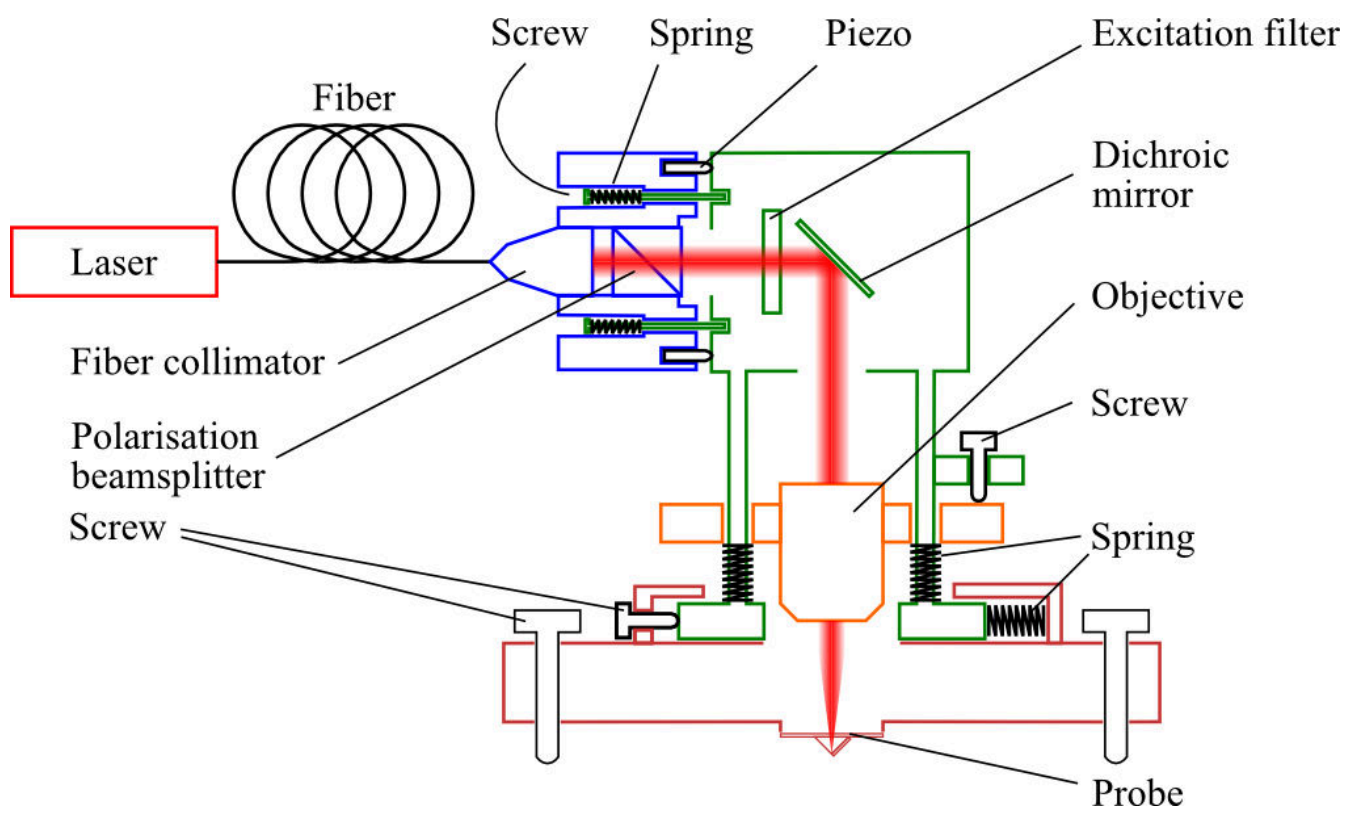

Figure 5.7.: Excitation setup to couple light into the tip of the near-field optical probe. Laser light is first coupled into an optical fiber then collimated. After purifying the polarization through a polarizing beam splitter and removing the background through an excitation filter, the collimated beam is focused upon reflexion at a dichroitic beam splitter by a microscope objective of long working distance. Coupling is usually done first by roughly translating the objective in the three directions using the dedicated micrometer screws. Fine coupling is achieved using the piezoelectric elements that are controlled by a voltage supply unit and allow a very precise inclination of the collimated beam, which results in precise translation movements in the focusing plane of the objective. The pieces of same color (apart from black pieces: springs, screws, piezos) are bound together.

The light source is the most basic but also the most important part of this excitation setup: it is delivered through a diode laser (PicoQuant $\mathrm{GmbH}$ ) with an intensity peak at $640 \mathrm{~nm}$ that can be operated in continuous wave (cw) mode for FCS measurements or pulsed mode for fluorescence lifetime measurements. This 
light source is then coupled into a single mode polarization maintaining optical fiber (Schäfter\&Kirchhoff GmbH) through a fiber coupler (Schäfter\&Kirchhoff GmbH).

At the other hand of the fiber a fiber collimator (Schäfter\&Kirchhoff $\mathrm{GmbH}$ ) transforms the laser beam into a collimated beam of $7.22 \mathrm{~mm}$ diameter (given at the $1 / \mathrm{e}^{2}$ value of the intensity maximum of the Gauss intensity distribution). The beam diameter was chosen so that the collimated beam completely illuminates the back aperture of the objective used to focused the light into the near-field optical probe, so that the best focusing can be obtained. The fiber collimator is mounted in a self-designed holder equipped with three piezoelectrical elements (PICMA PL055.30, PI Instruments GmbH) to enable an electronic control of the inclination of the collimated beam with high accuracy.

Once collimated, the beam passes through a polarization beamsplitter (Qioptiq) to obtain a precise linear polarization. Then an excitation filter (or clean-up filter) is used to filter out background light emitted either by the laser itself or by the fluorescent centers in the optical fiber upon excitation by the laser beam. This filtering is of utmost importance for FCS measurements with single molecules, since the fluorescence signal of these molecules can be buried under the background light at the detectors. Consequently, the useful dynamical information cannot be retrieved from the correlation of the fluorescence signal. For the FCS measurements, the excitation filter (HC Laser Clean-up MaxDiode 640/8, AHF Analysentechnik AG) does not efficiently reject background light above $700 \mathrm{~nm}$ although the photon detection efficiency of the detectors (see section 5.2) is still above $50 \%$ at $800 \mathrm{~nm}$. The efficient background suppression is in the case of this excitation filter only achieved in combination with an adequate emission filter (see section 5.2), a filter that only lets the main part of the fluorescence emission spectrum of the fluorescent molecules in solution through, but rejects higher or lower energetic light. The cumulated rejection efficiency out of the band of the emission filter from excitation and emission filters together is above optical density 6 (optical density OD $=-\log \left(\frac{I_{f}}{I_{0}}\right)$, where $I_{0}$ is the incident intensity light at a given wavelength on the filter and $I_{f}$ the filtered intensity).

The collimated beam is then reflected at a dichroic mirror (F53-530, AHF Analysentechnik AG) and focused by a microscope objective (ELWD Plan Fluor 20x, Nikon) with a high working distance of $7.4 \mathrm{~mm}$ in the tip of the near-field aperture probe. The numerical aperture of NA $=0.45$ enables to use nearly $40 \%$ of the whole opening angle of the tip $\left(90^{\circ}\right)$. A correction collar is also available at this objective to correct for the long path in glass $(2 \mathrm{~mm})$ that the light as to travel to the tip. The objective can also be roughly translated with three micrometer screws in the three directions. This is to achieve an approximate coupling of the light into the near-field probe. Precise coupling is achieved through the three piezoelectrical elements that can change the inclination of the collimated beam, which produces a translation of the focused beam in the focal plane of the objective.

Additionally, the near-field optical probe is fixed to a heavy stage (illustrated by the red block in figure 5.7) with the same glue used for the assembling of the 
glass pieces of the probe (see section 5.1.1). This way, possible infiltration of liquid from the sample is avoided (the near-field probe is immersed in the liquid). The assembly is placed on a small platform that can be moved with two micrometer screws in the horizontal plane. Fixed to the heavy stage, two manual micrometer screws and one motorized micrometer screw enable a rough positioning of the probe in the vertical direction.

Detection setup The samples on which FCS measurements are carried out lie on a scanning stage (P-517.3CL, PI Instruments $\mathrm{GmbH}$ ) that is fixed on an epifluorescence microscope (Eclipse TE-2000U, Nikon). The scanning stage enables a fine positioning of the near-field optical probe to the sample after the probe was roughly positioned with the micrometer screws of the heavy stage.

When light is coupled into the near-field optical probe the fluorescent molecules of the sample diffusing at the aperture emit light that is collected with the epifluorescence microscope, correspondingly to the light path illustrated in figure 5.8. The detection setup corresponds to a confocal detection scheme [14] where the detection fiber acts as a measurement pinhole.

The first optical element in the detection setup, an oil immersion objective ( $\mathrm{S}$ Fluor $100 \times$, Nikon) with high numerical aperture $(\mathrm{NA}=1.3)$ is used to gather fluorescence in a solid angle as high as possible. A coverslip of standard thickness $(170 \pm 5 \mu \mathrm{m})$ separates the water solution from the objective. The signal is detected at a maximal distance of $10 \mu \mathrm{m}$ away from the interface between coverslip and water, since the mismatch between refractive index of water and oil starts to disturb the light detection at the objective above this distance.

However, coupling light into the near-field optical probe also leads to emission in the far-field which is gathered together with the fluorescence light by the objective. In order to separate these contributions, the excitation light is filtered out through an emission filter (Bright Line R 685/40, AHF Analysentechnik AG). The tube lens of the epi-fluorescence microscope with a high focal length of $200 \mathrm{~mm}$ focuses the fluorescence light at one of the outputs of the microscope after reflection on a mirror. This light is finally focused with the help of a biconvex lens in the entrance of a multimode optical fiber (50/50 fiber divider, Schäfter\&Kirchhoff GmbH). This additional lens could appear superfluous, since the entrance of the optical fiber could be placed at the focal plane of the tube lens. However, this adds the possibility to integrate an additional light path for example to make an excitation setup with a confocal arrangement (see next section).

The optical fiber has a core diameter of $50 \mu \mathrm{m}$, which defines the pinhole size. Given the $100 \times$ magnification of the detection setup, the waist of the detection volume at the probe in the focal plane of the $100 \times$ objective is with $0.5 \mu \mathrm{m}$ nearly diffraction limited. Additionally, the optical fiber splits into two fiber elements for which the probability to transmit light is the same. Finally, the output of the pair of fibers are directed on a pair of avalanche photodiodes (APD) also called single photon avalanche diodes (SPAD) (SPCM-AQR-14-FC and SPCM-AQR-15- 


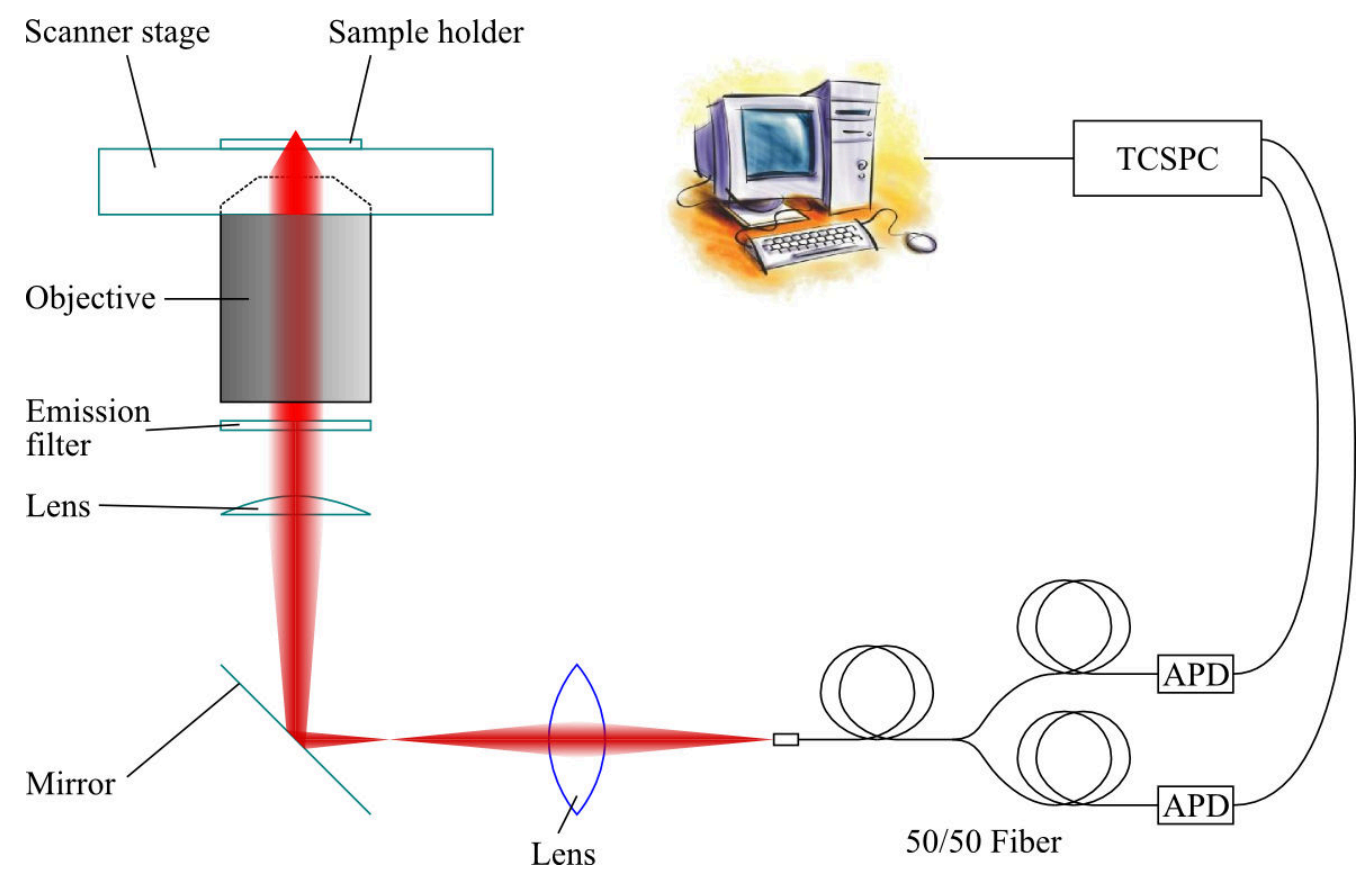

Figure 5.8.: The sample to study is placed on a sample holder fixed to a scanning stage. At the aperture of the near-field probe both excitation and fluorescence light are gathered from the objective of the epi-fluorescence microscope. Excitation light is separated from the fluorescence light with an emission filter. The tube lens of the microscope focused this light and a mirror directs it to one of the microscope output. Finally, a lens focuses the fluorescent light in an optical fiber that distributes the intensity to two outputs. The photon coming from these outputs are detected through avalanche photodiodes (APD) that emit TTL pulses upon detection. These TTL pulses are counted through a time correlated single photon counting device (TCSPC). The data are finally sent to a computer for the calculation of the cross correlation between the output signals of the two APDs. 
FC, PerkinElmer). To direct the output on the detectors, one lens is used for each pair fiber/detector so that the light spot at the detector illuminates the detector area $(175 \mu \mathrm{m})$ as much as possible. The TTL pulses emitted by the APDs are then detected at two different channels of a time correlated single photon counting device (TCSPC). A computer records the detection events counted by the TCSPC device and a correlation calculation can be carried out.

\section{Comparative FCS measurements with a confocal setup}

For comparative measurements, a confocal setup [14] was mounted at one of the side ports of the epi-fluorescent microscope (see figure 5.9). In this setup, part of the path taken by the excitation light is the same as the fluorescence light from the detection setup of figure 5.8. Similar to the excitation setup, the excitation light is provided by a diode laser (Sepia, PicoQuant $\mathrm{GmbH}$ ) and led to the confocal setup using the same optical fiber. The light is first collimated with a fiber collimator (Schäfter\&Kirchhoff $\mathrm{GmbH}$ ) and filtered through an excitation filter. Then this light is focused and directed in the epi-fluorescence microscope through a lens then followed by a silver mirror and a dichroic beamsplitter (AHF Analysentechnik AG). Finally, this excitation light is collimated again by the tube lens of the microscope and finally focused at the sample with the same objective that is used to detect the fluorescence (S Fluor $100 \times$, Nikon).

The fluorescence light stemming from the molecules being excited by this focused laser beam is gathered through the objective and then takes essentially the same light path and goes through the same optical component as in figure 5.8. Merely the emission filter has to be placed elsewhere: after the lens focussing the light in the detection fiber.

\subsection{Analysis of the fluorescence signal}

\subsubsection{Sample preparation}

The fluorescent molecules used to study the near-field distribution at a near-field optical probe are molecules that efficiently absorb in the red. The reason for this is on one side the too long penetration depth of green light in gold and on the other side the growing fluorescent background at blue shifted excitation wavelengths [70]. Therefore two red absorbing fluorescent molecules were chosen: Atto655 with free carboxyl group (ATTO-TEC GmbH) and Alexa647 carboxylic acid, succinimidyl ester (Molecular Probes, Invitrogen). The succinimidyl ester group is usually used to link the fluorescent molecule to a primary amine $\left(\mathrm{R}-\mathrm{NH}_{2}\right)$ but undergoes hydrolysis in aqueous solution to give the nonreactive free acid so that both Atto655 and Alexa647 show the same carboxyl group. The main difference between the two molecules lies in one of their photophysical properties: Alexa647 is believed to derive from the family of the carbocyanine dyes and therefore undergoes photoinduced isomerization at relatively small intensities of $7 \mathrm{~kW} / \mathrm{cm}^{2}[70]$. This results 


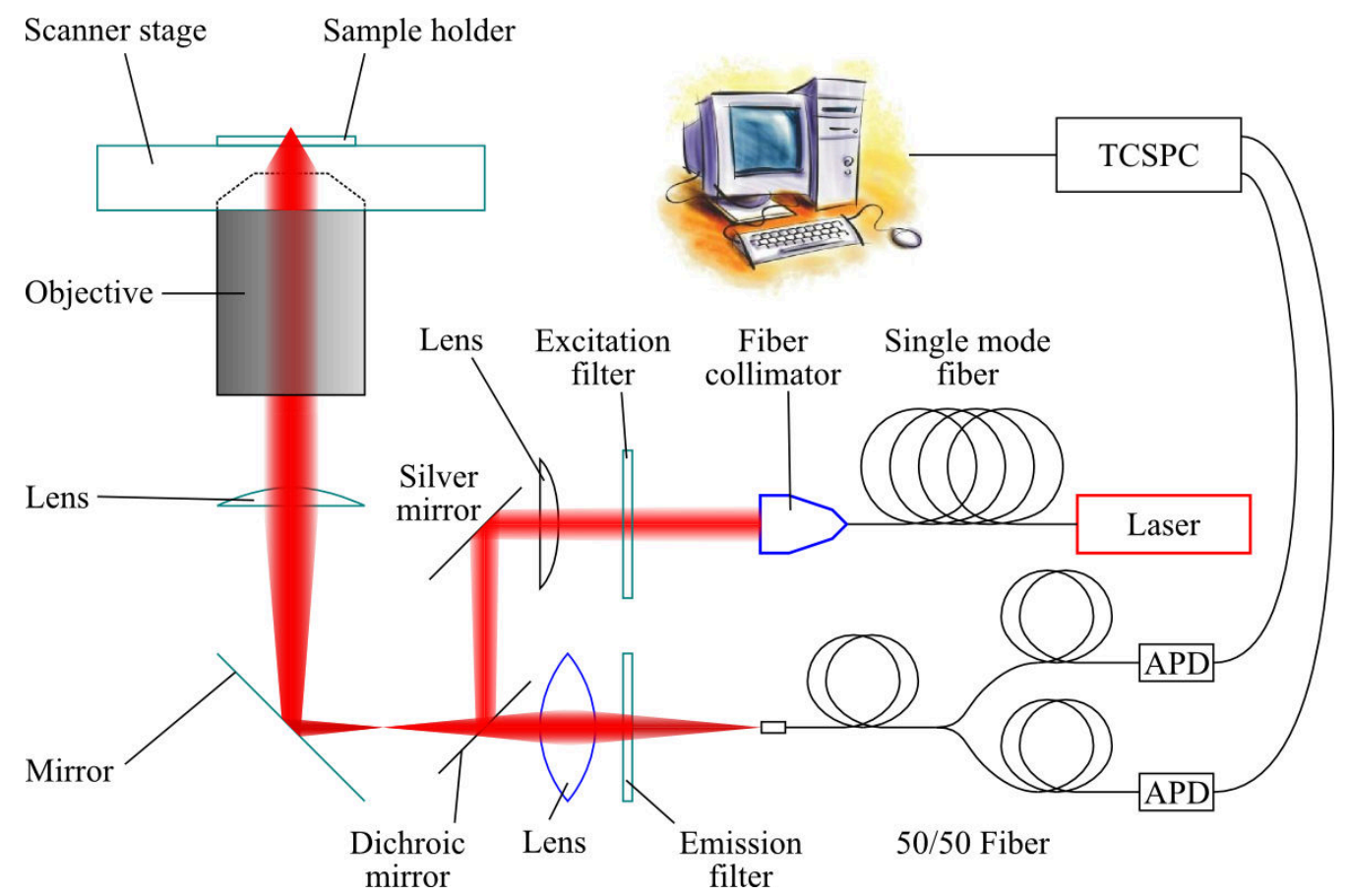

Figure 5.9.: The confocal setup is very similar to the detection setup used for the FCS measurements with a near-field optical probe (figure 5.8). Instead of exciting fluorescent molecules in solution through the probe, they are excited from a laser beam focused through the detection objective. The emission filter is correspondingly put out of the excitation light path and inserted just before the detection pinhole of the optical fiber. 
in the same feature on the autocorrelation function as for a molecule which triplet state can be excited. Atto655 does not show any triplet occupation at intensities as high as $100 \mathrm{~kW} / \mathrm{cm}^{2}[70]$.

To characterize an intensity distribution at a near-field optical aperture with FCS, the concentration of the fluorescent molecules must be chosen carefully, so that the amplitude of the correlation function that is proportional to the inverse mean number of molecules in the excitation volume is not too low and correlation times can still be derived from the correlation curve. Therefore, the purchased fluorescent molecules were always dissolved in aqueous solutions and diluted to concentrations from $100 \mathrm{nM}$ to $10 \mu \mathrm{M}$. First, the pure molecules purchased in solid form are diluted in ethanol (ROTIPURAN, $\geq 99,8 \%$ p.a., Carl Roth GmbH) at a concentration of about $1 \mathrm{mM}$. Afterwards a small amount of this solution is pipetted out and given to a big amount of water or buffer solution to achieve the desired concentration range. This way the amount of ethanol in the end solution is kept relatively low at typically $0,1 \%$. The water used for the dilution with or without buffer substance comes from a Synergy water purification system (DirectQ 3 UV Water Purification System, Millipore) with a resistivity of better than $18 \mathrm{M} \Omega \mathrm{cm}$ at $25^{\circ} \mathrm{C}$. For most buffered solution, phosphate saline buffer (PBS) was used and purchased as ready-to-use solution (Roti-Stock 10× PBS, Carl Roth $\mathrm{GmbH}$ ) with or without the deterging agent Tween20 (Roti-Stock 10× PBST, Carl Roth $\mathrm{GmbH}$ ). Also another ionic buffer (acetic acid / sodium acetate acid/base pair) and zwitterionic buffer like HEPES, CAPS and TRIS (all from Carl Roth $\mathrm{GmbH}$ ) were used in some measurements. The adjustment of the $\mathrm{pH}$ values were done with a $\mathrm{pH}$ meter (pH340i/SET, electrode SENTIx41) from WTW GmbH.

Once the solution with fluorescent molecules are ready, they are pipetted on a coverslip of thickness $170 \pm 5 \mu \mathrm{m}$ (Carl Roth $\mathrm{GmbH}$ ). This coverslip lies on a nickel coated aluminum plate with a hole in its center to allow light to get through. The coverslip is fixed to this plate with the help of small magnets (Peter Welter $\mathrm{GmbH}$ ). The plate itself is also fixed with magnets to the scanning stage of the epifluorescence microscope. The coverslip are either taken from the packaging without further treatment or treated with a $1 \%$ solution of Hellmanex II (using the same protocole as for the cleaning of the glass pieces used for near-field probe fabrication). As this cleaning solution causes the surface to become highly hydrophilic $1 \mathrm{~mm}$ high and $2 \mathrm{~mm}$ large borders made out of polydimethylsiloxane (PDMS, Sylgard 184 Silicone Elastomer Kit, Dow Corning) are glued (also with PDMS) to the coverslip. This is done by first mixing the silicone base to the the curing agent in proportion $10: 1$ and storing the mixture under vacuum to allow it to outgass. The viscous liquid mixture is poured in a teflon mould and a small magnet $(2 \times 2 \times 1 \mathrm{~mm})$ is added in each corner of the PDMS border [82]. The whole structure is then put in an oven to dry at $70^{\circ} \mathrm{C}$ for one hour. Finally, the $1 \mathrm{~mm}$ high borders are glued once again with the same or a fresh PDMS mixture and the complete structure is put to dry once again in the oven under the same conditions as before.

The amount of solution with fluorescent molecules used to carry out the FCS measurements is about $200 \mu \mathrm{L}$. No precaution is taken regarding the vaporization 
of the solution during the measurements and its impact on the amplitude of the correlation curve (typically decreasing with time as a result of a higher density of fluorescent molecules in solution) as the near-field optical probe is dived into the droplet of solution and in doing so the solution wets nearly the whole probe and part of the probe holder, which results in a much smaller water-air interface and therefore reduces the problem of evaporation.

Finally for the application of FCS with near-field optical probe to the measurements of dynamical biological processes, a microstructured template was used to allow patches of a biological membrane to stand freely and allowing a solution to be on both sides of the membrane. The structure was developed by Jörg Wissler and consists of an array of holes of $20 \mu \mathrm{m}$ diameter and $2 \mu \mathrm{m}$ in depth in a transparent photoresist lying on a coverslip [82]. The fabrication process starts by cleaning the coverslips in a piranha solution with one volume part of concentrated sulfuric acid (Carl Roth GmbH) and two volume parts of hydrogen peroxide (30\%, Carl Roth $\mathrm{GmbH}$ ). The coverslips are held in a glass holder and dived in this fresh mixture for 30 minutes by simultaneous stirring with a magnetic stir bar embedded in a glass matrix. The very corrosive piranha solution has to be handled with care and all the pieces staying in contact with the solution for a longer time are in the ideal case made out of glass. During the first 15 minutes the solution is very hot (above $100^{\circ} \mathrm{C}$ ) due to the strong exothermic reaction between the two compounds but after this period the solution is placed on a hot plate and is heated at $150^{\circ} \mathrm{C}$ for further 15 minutes. After the coverslips are rinsed with a copious amount of water they are ready to be coated with the negative photoresist (SU8-5, Microchem Chemicals). The coating is done on a spin-coater by giving $500 \mu \mathrm{L}$ of the SU8-5 solution and starting the spinning at 400 revolutions per minute (rpm). After 10 seconds the spinning is accelerated to $4000 \mathrm{rpm}$ for the last 50 seconds. After spin coating a soft bake step is carried out on a hot plate at $65^{\circ} \mathrm{C}$ for one minute. This is to evaporate the solvent of the photoresist and densify the film. At this point the photoresist is ready for exposure. This is done with a UV lamp (UVAHAND, Dr. Höhnle $\mathrm{AG}$ ) and a microstructured chromium mask for 45 seconds. After the exposure, the coverslip with photoresist goes through a post exposure bake step at $100^{\circ} \mathrm{C}$ for 5 minutes to optimize the cross-linking process initiated during the exposure. Finally, the photoresist that is not cross-linked is removed with MicroChem's SU8 developper (xr600, Microchem Chemicals) by diving the coated coverslip 3 times successively into the chemical for a few seconds. After drying the coverslips with the microstructured array under a gentle nitrogen flow, PDMS borders are glued on the coated coverslip as described before.

\subsubsection{Auto- and cross-correlation calculation from the detected signals}

As shown in the detection part of the setups in figure 5.9 and 5.8, the fluorescence signal is not detected by one fiber and one detector but with a fiber that 
distributes the input intensity in two outputs of half intensity ("50/50 fiber") that are connected at the input of two APDs. The reason for using a 50/50 fiber and two detectors is to get rid of two drawbacks in FCS measurements with one detector: Firstly, an avalanche photodiode has a finite dead time, i.e. as it detects a photon and accordingly emits a voltage pulse (a TTL pulse $2.5 \mathrm{~V}$ high in a $50 \Omega$ load and $30 \mathrm{~ns}$ wide) it cannot detect any other photon for a defined period after the detection. In the case of the detector employed in this work, this dead time is between 50 and 60 ns. Additionally the channels of the TCSPC device have also a dead time of $90 \mathrm{~ns}$. Consequently a photon detection leads always to no photon detection in the next period of time corresponding to the dead time. An artificial anticorrelation appears in the auto-correlation curve that has nothing to do with a correlation in the fluorescence signal. The second drawback stems from the so-called afterpulsing of such detectors: normally the detector emits a TTL pulse if a photon impinges on the detection area (above $70 \%$ detection efficiency in the domain $650-750 \mathrm{~nm}$ ). However, there is also a small but finite probability that another voltage pulse follows the first one. This phenomenon is called afterpulsing and even if its probability is typically as low as $0.3 \%$, it is still significant enough to produce a correlation visible in the auto-correlation function of the electrical signal at one detector. This afterpulsing probability is only significant for lag times below $1 \mu$ s, i.e. if no second voltage pulse was emitted in the microsecond following the first detection pulse, the probability for the detector to emit a second voltage pulse at a later time is negligible. But it also means that correlations in the fluorescence signal for lag times smaller than $1 \mu$ s are burried under this artificial positive correlation.

These drawbacks are not problematic as long as FCS measurements with smaller lag times as $1 \mu \mathrm{s}$ are not necessary. On the contrary, a cross-correlation of the signals of two detectors enables one to measure correlation in a smaller time range: if a photon is detected at one detector, the other detector is very likely to be ready to detect a photon directly afterwards. However, this requires a photon pair to split in the optical fiber at the output, which occurs only with $50 \%$ probability. Therefore for time ranges where dead time and afterpulsing are no issue, a cross-correlation of two signals stemming from one splitted fluorescence signal features a lower sample size for the statistical distribution of correlation events than an auto-correlation of the same unsplitted fluorescence signal. For correlation in a time range under $1 \mu \mathrm{s}$ a cross-correlation of the signal of two APDs has to be favoured to an autocorrelation. Owing to the small excitation volume expected at a near-field optical probe and the corresponding fluctuations in fluorescence in a very small time scale, the cross-correlation measurement scheme has to be adopted for near-field optical FCS.

However, there is a drawback to the use of a 50/50 fiber. The cross-correlation function are characterized by the presence of a peak at a lag time of about $30 \mathrm{~ns}$ (see for example figure 7.5). This correlation is actually an artificial one that stems from the detection setup shown in figure 5.8. Upon detection of a photon, the detector can emit a photon of longer wavelength that gets into the 50/50 fiber. 
This photon has a small probability of being reflected at the other side of the fiber and gets the way back to one of the two detectors. The detector that emitted this photon is still in a state where it cannot detect further photon (dead time) but it is highly probable that the other detector can detect this photon. When it does, it leads to a correlation in the signal that is therefore artificial and has no relevance for the fluctuations of the "true" fluorescence signal. However, this impairs the cross-correlation function of this fluorescence signal stemming from the fluorescence molecules and can cause difficulty in the analysis of the curve. This can be removed by adding an additional emission filter between the output of the 50/50 fiber and the detector. This effect tends also to disappear at higher count rates because either of the detectors are more likely to be in their dead time when the other emits the photon leading to the artificial correlation and therefore cannot detect it.

The TTL pulses marking the detection of a photon at each detector (and possibly some additional pulses due to afterpulsing) are detected at the TCSPC device in Time-Tagged Time Resolved mode (TTTR). This TTTR mode realizes a precise timing of the electrical pulse detection: at the beginning of the measurement, the time is set to 0 . When a pulse is detected, the arrival time $t_{i}$ (called time stamp) of this pulse is recorded together with the detection channel (giving one channel for each detector). The advantage of such a recording procedure is its memory efficiency. Instead of sampling time and recording if in this time interval a photon was detected or not, which for a time resolution of $1 \mathrm{~ns}$ and a measurement period of $1 \mathrm{~s}$ would require a total number of $10^{9}$ samples with many sampling times having no detection events, only the time arrival of detected photon and their detection channel are recorded and transmitted to the computer. This data flow to the computer is processed through a home-made software enabling a calculation of the auto- or cross-correlations of the signals in real-time up to a cout rate of some $10^{5} \mathrm{Cts} / \mathrm{s}$.

The calculation of the auto- and cross-correlation are based on the multiple-tau algorithm [83]: instead of being linearly spaced, the lag times $\tau$ in equation 3.26 are logarithmically spaced. To compute the correlation function, the arrival time $t_{i}$ recorded by the TCSPC device in TTTR mode are first converted to photon counts with sampling time $t_{s} . n_{j}$ corresponds to the number of photons counted in the time interval $(j+1) t_{s}-j t_{s}$. This way, a list $\left\{n_{j}\right\}$ of photon counts can be built for the whole measurement time $T_{\text {tot }}=M t_{s}$, where $M$ corresponds to the number of sample times. Using this list the correlation function can be calculated for the lag time $\tau_{s}=k t_{s}$ with [84]:

$$
G\left(\tau_{s}\right)=M \frac{\sum_{j=1}^{M} n_{j} n_{j-k}}{\sum_{j=1}^{M} n_{j} \sum_{j=1}^{M} n_{j-k}}-1
$$

This calculation is eventually repeated for all the logarithmically spaced lag times 
to give the final correlation function. Note that the sample time $t_{s}$ are also increased with increasing lag times $\tau_{s}$, so that the number of sample times $M$ decreases with $\tau_{s}$. 


\section{Near-field optical microscopy combined with FCS: characteristics and applications}

In this chapter, the advantages and disadvantages of NSOM-FCS are discussed in comparison to other common techniques. Following this, its application to the measurement of dynamic processes at a biological membrane under physiological conditions is shown.

\subsection{Comparison of the combination of NSOM probes with FCS over other combinations}

\subsubsection{Time resolution}

One of the most important aspects for a single molecule technique is its time resolution, i.e. the shortest characteristic time of a dynamical process that it is able to measure. This time is limited by the mean time it takes the molecules needs to diffuse in and out of the excitation volume. This time can be assessed with the FCS measurement of fluorescent molecules that are freely diffusing in the solution without being involved in other dynamical processes such as binding or chemical reactions. The shorter this time, the easier it is to distinguish free diffusion of fluorescent or dye labeled molecules from other dynamic processes. This time resolution is examplified in figure 6.1, where FCS simulations were carried out for different common single molecule techniques and are shown together with an NSOM-FCS measurement. In order to compare the different methods, all the correlation functions were normalized. The time resolution can then be approximated by the time at which the correlation function has decreased to 0.5. This definition corresponds exactly to the mean diffusion time $\tau=R_{x y}^{2} / 4 D$ in the case of a two dimensional diffusion in a Gaussian excitation profile [65].

FCS using a confocal setup was the first technique used to measure the dynamics of single molecules [65]. The FCS simulation corresponding to an excitation volume with dimension $R_{x y}=250 \mathrm{~nm}$ and $R_{z}=750 \mathrm{~nm}$ is shown on the right part of the graph. For these quite realistic dimensions, the time resolution is about $33 \mu \mathrm{s}$. This time resolution is about $10 \%$ smaller than that of a two dimensional diffusion in a Gaussian excitation profile with the same characteristic lateral dimension $R_{x y}$. 


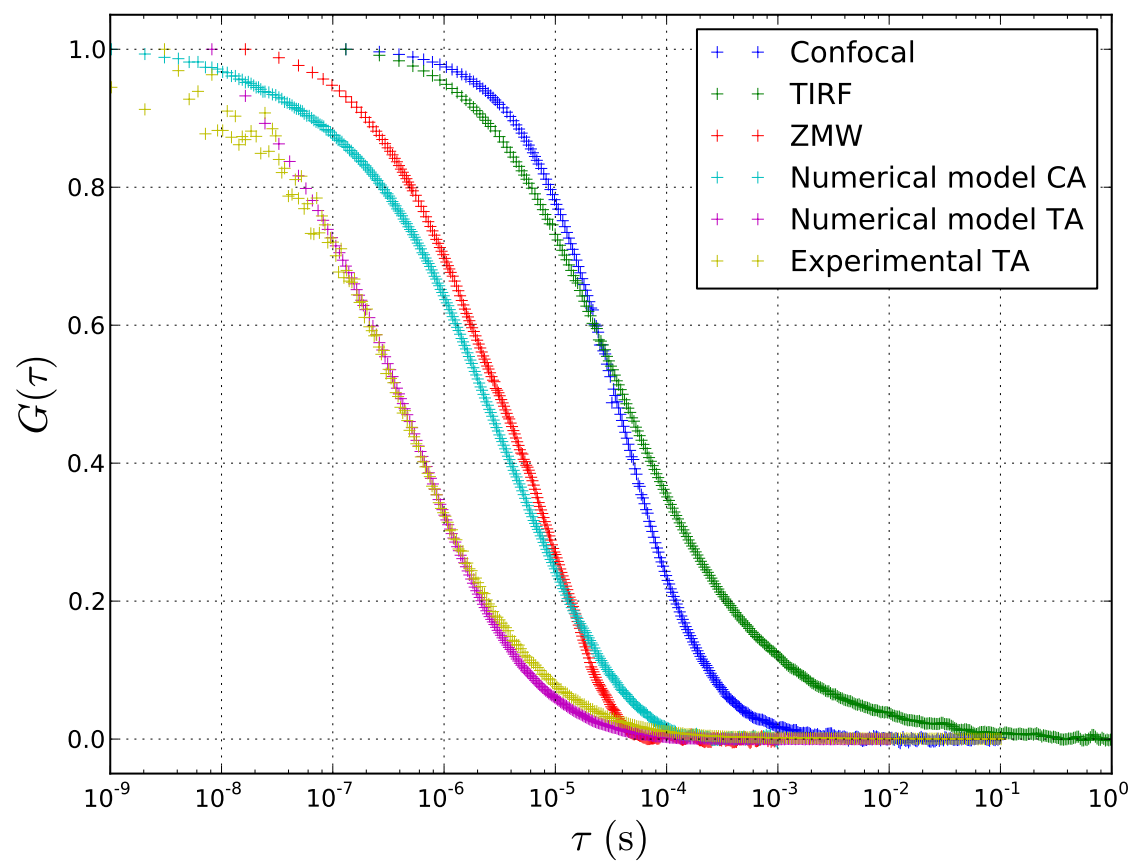

Figure 6.1.: Time resolution of different combinations of FCS and single molecule techniques. For FCS using a confocal setup (blue curve), a Gaussian excitation volume with $R_{x y}=250 \mathrm{~nm}$ and $R_{z}=750 \mathrm{~nm}$ was used. In the case of TIRF-FCS (green curve), the intensity distribution is homogeneous in the lateral plane and decays exponentially with a decay length of $70 \mathrm{~nm}$ axially. The FCS simulation using Zero-Mode Waveguide (ZMW, red curve) used holes of $50 \mathrm{~nm}$ in diameter and $100 \mathrm{~nm}$ in length. The intensity distribution inside the hole is assumed homogeneous in the lateral plane and decreases exponentially with growing distance to the hole ground with decay length $d=20 \mathrm{~nm}$. Using the numerical model used to calculate the intensity at an aperture in a metal film (section 4.2.2), FCS was simulated for a circular aperture (CA) of $80 \mathrm{~nm}$ diameter (cyan curve) or a triangular aperture (TA) of $80 \mathrm{~nm}$ side length (magenta curve). Additionally a typical FCS measurement with a triangular aperture is shown for comparison (yellow curve). All the correlation functions are normalized. 
A few years later, another single molecule technique was introduced in combination with FCS: TIRF [21]. This technique allows to reduce the confinement of the intensity in axial direction by using evanescent waves excited by total internal reflection (TIR, see figure 4.7). In such a setup, the intensity distribution is homogeneous in the lateral plane. In figure 6.1, an FCS simulation using this setup is shown where the collection efficiency function (CEF, see equation 3.32) is also homogeneous in the lateral plane. The decay length of the intensity was set to $d=70 \mathrm{~nm}$, which is a typical value for TIR [21]. In this case, the time resolution of about $38 \mu \mathrm{s}$ is comparable to that using a confocal setup. It is possible to further reduce this time resolution by introducing a pinhole in the image plane of the objective that collects fluorescence, so that the CEF is not homogeneous anymore. This speeds up the decay of the correlation function and consequently a smaller time resolution is achievable [21]. However, this resolution is at most one order of magnitude smaller than that of FCS using a confocal setup.

A promising new technique within the family of single molecule techniques is Stimulated Emission Depletion (STED) which uses a particularly shaped laser beam to deplete the fluorescence at the outer part of a focused laser beam and to achieve sub nanometer resolution [10]. This technique has already been used to obtain very high resolved images of fluorescent nitrogen vacancies in diamond with a spatial resolution of $5.8 \mathrm{~nm}$ [85]. Its implementation for FCS shows, however, a minor increase in time resolution of about 1.5 [22]. This is due to the fact that mostly the axial diffusion time is reduced ( 25 fold) but this diffusion time does not weigh as much as the lateral diffusion time in the correlation function (see equation 3.53).

Zero-Mode Waveguide (ZMW) has been introduced for nearly a decade in order to measure dynamics of single molecules at concentrations in the micromolar range [24]. For FCS at these concentrations using a confocal or a TIRF setup, the amplitude of the correlation function decreases under the noise level and no information on the dynamics of the single molecules can be retrieved. ZMW are arrays of subwavelength holes in a metal film coated on a glass surface. These holes are usually fabricated by electron beam lithography and subsequent reactive ion etching on an aluminum coating coverslip [24] or directly by milling the holes in the aluminum film using a focused ion beam [16]. At the bottom of the holes only evanescent waves exist with a much smaller decay length than that used in TIRF. Additionally, the intensity distribution in the hole in a plane parallel to the coverslip is almost homogeneous when using circular polarized light [24]. A simulation of FCS using a hole of diameter $50 \mathrm{~nm}$ in a $100 \mathrm{~nm}$ thick metal film is also shown in figure 6.1. The decay length of the intensity in the axial direction was chosen so that the FCS curve approaches those given in [24]. It results in a decay length of about $20 \mathrm{~nm}$. It should be noted, however, that this does not correspond to the decay length of the excitation intensity but that of the overall fluorescence emission profile. This profile also takes into account the approximately exponential decrease of fluorescence collection with growing distance of the fluorescent molecule to the ZMW ground. The resulting time resolution is about $3 \mu \mathrm{s}$, which is an order of magnitude better than TIRF. 
Similarly to ZMW, a circular aperture at the apex of a metal coated glass tip such as in the case of a fiber probe allows one to measure dynamics of single molecules with a high time resolution of about $2 \mu \mathrm{s}$. This is the result of an FCS simulation using the intensity distribution calculated from a similar model as that used for the triangular aperture in section 4.2.2. Only, the shape of the aperture was modified to a circular one with a diameter of $80 \mathrm{~nm}$. The corresponding FCS simulation is shown in figure 6.1.

Using a triangular aperture in a metal film results in a nearly one order of magnitude better time resolution than in the case of a circular aperture or a ZMW. An experimental measurement confirms this very small time resolution of about $300 \mathrm{~ns}$, that is the smallest resolution among the techniques presented here. Today, there are no other techniques offering a better time resolution. This resolution is so small that it falls under the typical dead time of typical avalanche photodiode detectors $(1 \mu \mathrm{s})$. Even smaller time resolution of about $200 \mathrm{~ns}$ are possible for some probes as shown from experimental measurements in section 7.4.

\subsubsection{Excitation volume}

Excitation volume and time resolution are parameters that depend on each other. For a given intensity profile in each direction, if the characteristic length of this profile ( $R_{x y}$ for a Gaussian profile, $d$ for an exponential decaying intensity) decreases, then the time resolution decreases. However, the situation is a little different if homogeneous profiles in some directions exist. If the molecules are not allowed to diffuse in and out of the excitation volume in the direction for which the intensity profile is homogeneous, then the time resolution does not vary with the extent of the homogeneous intensity profile, whereas the excitation volume does.

In order to illustrate this, the five simulations presented in figure 6.1 were scaled so that the concentrations of molecules used for the FCS simulations of the different techniques all correspond to the concentration used for the measurement given in the same figure ("Experimental TA"), i.e. $250 \mathrm{nM}$. This scaling is possible since the process of fluorescence fluctuation due to molecular diffusion is linear with the number of molecules $(G(0)=1 / N)$. Since $N=C_{0} V_{\text {exc }}$ and $C_{0}$ is kept constant ( $250 \mathrm{nM}$ corresponds to $C_{0}=150.5 \mu \mathrm{m}^{-3}$ ), the relative size of the excitation volume for the different techniques can be easily compared with one another.

The comparison of the amplitudes for the FCS simulation using the different techniques given in the precedent section shows the superiority of ZMW. Its excitation volume for a typical hole of $50 \mathrm{~nm}$ in diameter and for a decay length of the intensity of $20 \mathrm{~nm}$ is nearly a factor 30 smaller than the excitation with the triangular aperture probe with side length of $80 \mathrm{~nm}$ and over three orders of magnitude smaller than in a confocal setup, compatible with [24].

The FCS measurement with a triangular aperture probe is consistent with the corresponding simulation. Only the amplitude is a little smaller of a factor two. The rim size for this aperture probe was about $115 \mathrm{~nm}$, i.e. quite larger than that used for the simulation. Therefore, a smaller excitation volume could be expected using 

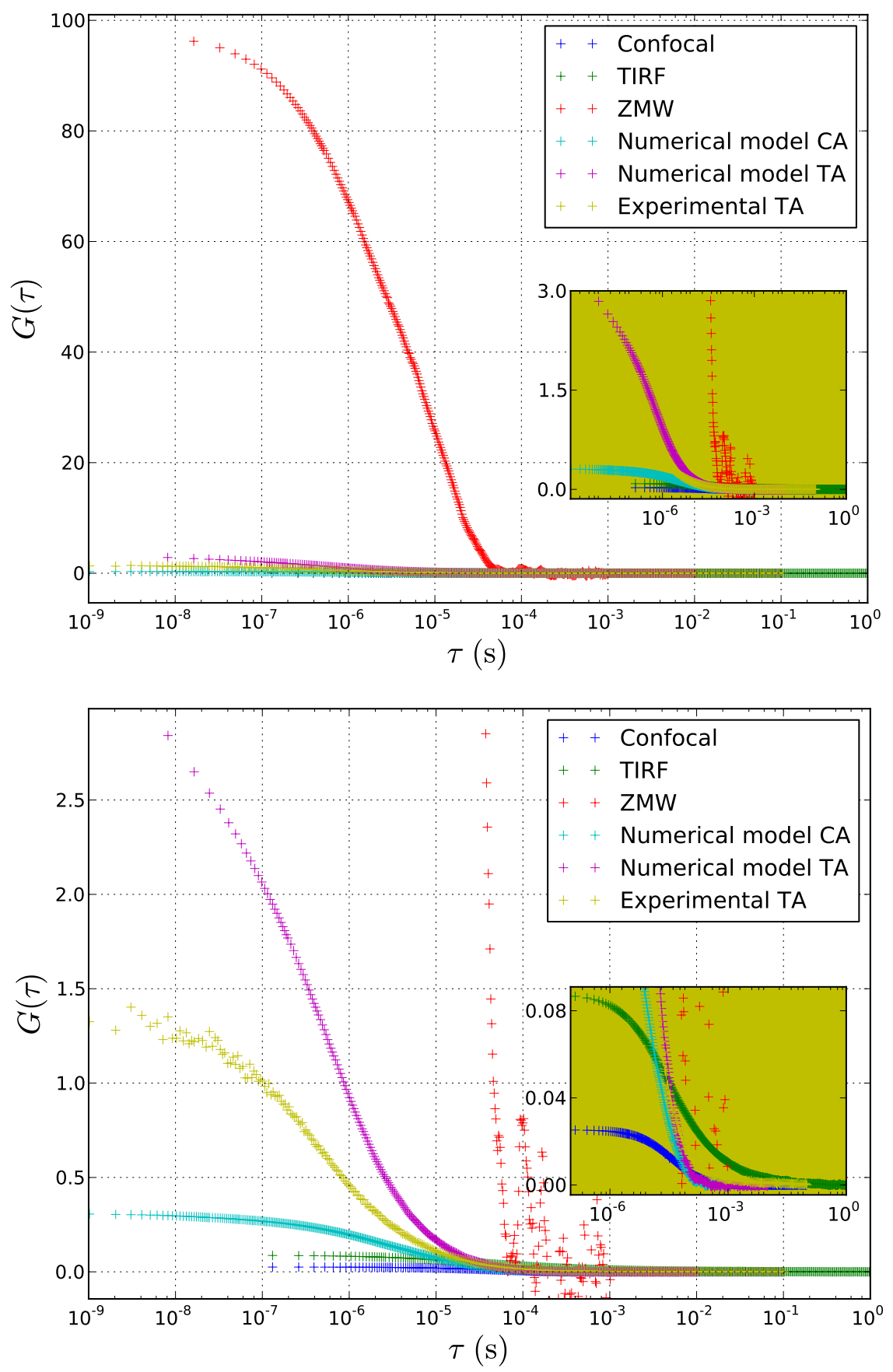

Figure 6.2.: The same FCS simulations presented in figure 6.1 were scaled so that the amplitude at zero lag time, $G(0)=1 / C_{0} V_{\mathrm{exc}}$, corresponds to the same molecules concentration $C_{0}$ used for the FCS measurement of the same figure ("Experimental TA"). This FCS measurement was taken with Atto655 molecules at a concentration of $250 \mathrm{nM}$ and with a gold coated aperture probe with a rim size of about $115 \mathrm{~nm}$. The two graphs with insets present the same curve at different scale for a better visibility. 
smaller rim size. However, for FCS measurements, the relation between amplitude of the correlation function and size of the excitation volume is more complicated and needs to be further analyzed (see section 7.2).

The slower decaying fields in a TIRF setup leads to a much larger excitation volume than that of an aperture probe and hardly achieved in the simulations a five fold decrease of this volume compared to the focused Gaussian beam with diffraction limited resolution. However, experimentally, a decrease of one order of magnitude is typical, probably due to a larger excitation volume in a confocal setup than the theoretical Gaussian excitation volume.

\subsubsection{Application domain and invasiveness}

NSOM-FCS is first and foremost a surface sensitive technique, similar to TIRF or ZMW. It does not have the ability of probing dynamics of fluorescent molecules at distances more than a few ten nanometers away from the aperture of the near-field optical probe. It has the advantage over a TIRF setup that the intensity decays much quicker in both the axial and lateral direction, which leads to a far better time resolution and a much smaller excitation volume. On the other side, TIRF-FCS is easily implemented and the samples can quickly be exchanged without modifying the excitation setup [86]. This is for example helpful for biological applications, where buffer solutions can be rapidly exchanged. Also the vaporization of buffer solutions can be efficiently prevented, so that biological samples always stay hydrated and conserve their physiological relevance.

FCS with ZMW is also a highly surface sensitive technique and its main advantage consists in the very small observation volume and the efficient background suppression due to fluorescent molecules out of a ZMW. This allows one to measure dynamics at concentrations as high as $20 \mu \mathrm{M}$ [24]. Similarly to near-field optical probes, ZMW relies on the quality of fabrication of the nanostructure. For NSOMFCS, small differences at the nanoaperture can lead to different or unexpected behavior in the FCS simulations (see section 7.1). Although ZMW has to deal with the same fabrication complexity, its fabrication can be highly parallelized by using a large array of small holes. This way, an efficient statistical analysis can help to filter out some FCS measurements as outliers and better describe the influence of the size of the nanostructure on the FCS measurements and consequently on the excitation volume. A major problem of ZMW for the measurement of dynamic processes at biological membranes is the necessity for the membrane to spread onto the bottom of the hole. This requires a high curvature of the membrane at the entrance of the ZMW that can be detrimental for the functionality of the membrane. At the expense of a complexer fabrication, this issue was removed recently by filling the ZMW with a dielectric [87]. However, the membrane then lies quite far away from the bottom of the hole and therefore, the high excitation intensity there is much weakened at the top of the hole, which results in a smaller signal from the fluorescent molecules, whereas the background signal increases by detecting the fluorescence above the holes. 
Despite its relatively small time resolution and excitation volume, the combination of FCS with a confocal setup is still the most popular among the here discussed techniques [88]. It is not only non invasive and easy to implement using epi-fluorescence microscopes. It can also be used to probe with diffraction limited resolution a large region of the sample of interest. Using water immersion objectives, biological samples can be probed over $100 \mu \mathrm{m}$ thickness compared to about $100 \mathrm{~nm}$ and $10 \mathrm{~nm}$ for TIRF-FCS and NSOM-FCS, respectively.

With the same advantage of large field depth, STED-FCS achieve a better resolution with theoretically no lower bound. However, the optical setup is more complex and an index mismatch inside cells leads to difficulties to deplete the excited molecules in the outer part of the excitation spot. If this is not perfectly realized, a high fluorescent background decreases the amplitude of the correlation function and diminishes the practical applicability to measurements with a high concentration of fluorescent molecules [22]. Also, the high power density needed for the depletion beam $\left(10-100 \mathrm{MW} / \mathrm{cm}^{2}\right)$ raises concerns about fluorescence photobleaching and the phototoxicity for the cell [26].

For NSOM-FCS photophysical effects are not an issue since the power density is very small at the probe aperture because of a large absorption and reflection of light in and at the metal coating. Moreover, fluorescence quenching and fluorescence enhancement both decrease the fluorescence lifetime and consequently the mean residence time of a molecule in an excited state. Furthermore, the molecules spend less time in the excitation volume because of its smaller size compared to other techniques. Consequently, the molecules are unlikely to make a transition in the triplet state and unlikely to be destroyed by triplet-triplet annihilation processes (see section 3.1.2). However, the smaller power density at the probe aperture can be an issue if the emitted fluorescence signal is too small. This is why near-field aperture probe fabricated by thermal pulling must have an aperture large enough. Using the triangular aperture probe with their high taper angle is an incontestable advantage for high fluorescence emission signals. As an additional consequence of the high light throughput of a triangular probe, a smaller aperture size can be used, which opens up the way to spatially high-resolved FCS measurements.

Owing to the fast decaying intensity at the near-field optical probe, the probe has to be brought in close proximity to the sample. NSOM-FCS is therefore an invasive technique such as TIRF or ZMW. For measurement at biological membranes with these probes, a shear force feedback is usually used to ensure a distance of the sample to the probe of a few nanometers [25], [26]. However, the quality factor of the resonance of the piezoelectric element used for this feedback decreases in water, which makes it difficult to reliably control the probe-sample distance. In this work, this active feedback was abandoned and consequently the possibility to measure FCS at samples lying on a hard surface such as glass. Instead, a passive force feedback was implemented using the tension of a free standing membrane spanned over a hole (see section 6.2.1). Using such a passive force feedback requires the fabrication of an adequate sample preparation, mainly by the design of the sample holder. However, the complex sample preparation is counter balanced by an easier 
probe preparation since the probe does not need to be attached to a piezoelectric element. For NSOM measurements with active force feedback the probe has to be light weighted enough in order to not disturb the resonance of the piezoelectrical element. Without this demand, the near-field aperture probe can be prepared with more flexibility.

Compared to FCS with ZMW, NSOM-FCS has the particular advantage that the probe can be used to scan the sample and consequently image the sample. This way, a defined place on the sample can be chosen for a dynamic measurement and the probe can be moved at this place for a subsequent FCS measurement. This is particularly useful for transport measurements through a biological membrane, that occurs at defined sites called "pores". In the case of a ZMW array and for diffusion measurements through a membrane, such imaging is not possible. Instead, FCS measurements have to be taken at many ZMWs and a post imaging process using for example electron microscopy has to be used in order to identify if the desired structure at which the diffusion process takes place lies over a hole and identify this hole with an FCS measurement realized before this post imaging process [82].

After this section outlining the strength and weakness of NSOM-FCS, an application to the measurement of the translocation dynamic at a biological membrane is shown in the next section.

\subsection{Application example of NSOM-FCS for the measurement of fast biological processes on the nanometer scale}

This section shows how the triangular aperture probes developed in this thesis were used to measure dynamical processes at a biological membrane (translocation) at nearly physiological conditions. Additionally, it shows how useful information on the translocation dynamics can be extracted by using a simplified model of the intensity distribution at the aperture adapted to the particular characteristics of the structure at which the translocation takes place. The following chapter mainly follows the description in [89]

\subsubsection{Imaging of single nuclear pores in a nuclear membrane}

NSOM with an aperture probe developed in this work was applied to study the kinetics of protein transport through a membrane. To our knowledge, this was never done before, presumably because common techniques relied on a support for the membrane and thereby obstructed the natural passageway. By establishing a novel method for NSOM imaging on unsupported membrane patches, we aimed at observing the gated transport through nuclear pore complexes (NPCs) embedded in the nuclear envelope (NE). NPCs are highly differentiated macromolecular assemblies of ring-like structure that regulate the exchange of molecules between nucleus 
and cytoplasm (see figure 6.3) [90], [91], [92], [93], [94]. Small molecules are allowed to diffuse freely through the central transport channel whereas the translocation rate of larger molecules $(>30-40 \mathrm{kDa})$ depends greatly on their interaction with NPC proteins containing natively unfolded phenylalanine-glycine-repeat domains (FG-repeat nups).

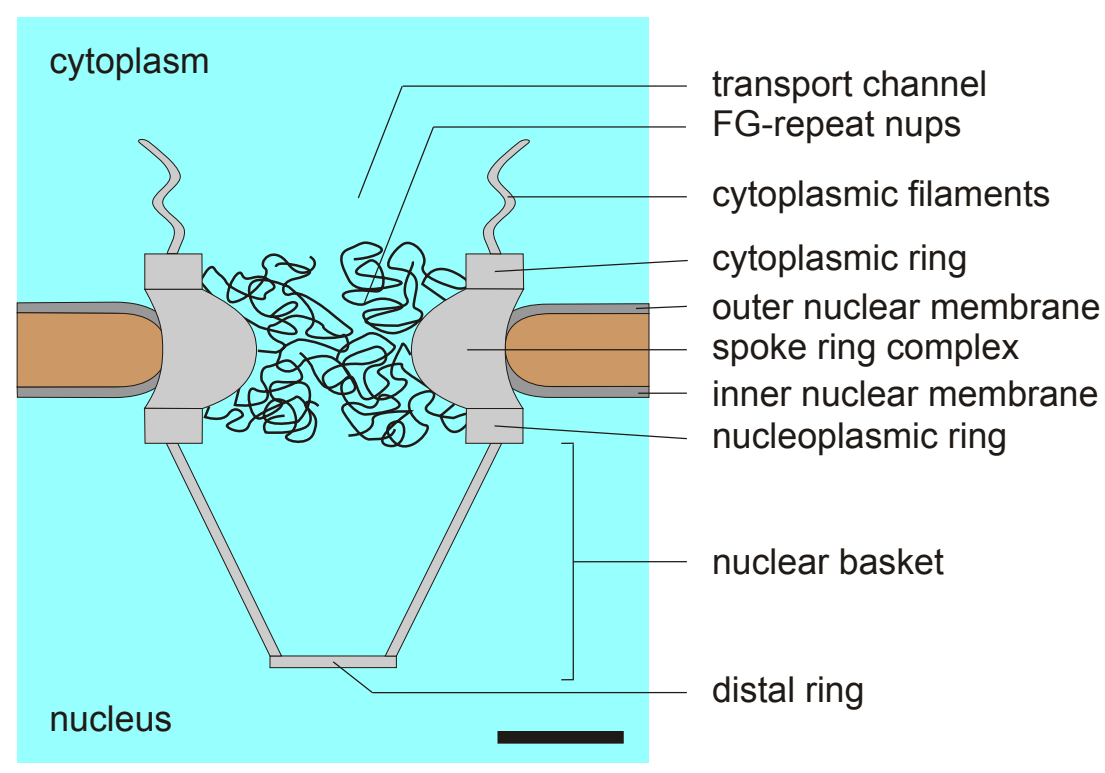

Figure 6.3.: Schematic of a vertebrate nuclear pore complex (NPC). The NPC is anchored at the merge of the outer and the inner nuclear envelope (NE). The cylinder-like structure of the NPC exhibits an octagonal symmetry with an outer diameter of $120 \mathrm{~nm}$. At the cytoplasmic side eight 35-nmlong flexible filaments emanate from the cytoplasmic ring, while from the nucleoplasmic ring another eight filaments extend $60-75 \mathrm{~nm}$ into the nucleoplasm and join to a distal ring forming a basket-like structure [93]. The spoke ring complex together with the cytoplasmic and nucleoplasmic ring moiety build the central framework $(50-90 \mathrm{~nm}$ in length) and enclose the hourglass-shaped pore with an inner diameter of 45-50 nm in the center [92], [93], [94]. Phenylalanine-glycine-repeat proteins (FG-repeat nups) line the transport channel and play a major role in the gating mechanism of the size-selective transport barrier [95]. Presumably, transport factors interact with natively unfolded FGrepeat domains for translocation of cargo molecules through the NPC [90], [91], [92]. Scale bar, $40 \mathrm{~nm}$ [89].

Here, we introduce a method that allowed us to employ the benefits of NSOM, ultra-high spatial and temporal resolution, on unfixed biological membranes in physiological solution. Application of this method to an unsupported native NE enabled us to resolve NPCs densely packed in the membrane with sub-50-nm optical resolution. After placing the near-field probe over a single NPC, we were able to 
observe the rapid translocation of fluorescence-labeled Nuclear Transport Factor 2 (NTF2) [96], [97] and gathered previously inaccessible kinetic information about a molecule passing through a NPC. By monitoring the fluorescence of NTF2 near the entrance of the transport channel and by analyzing the fluctuations using FCS we characterized the translocation of NTF2 as driven by Brownian motion and obtained values for the apparent diffusion coefficient, the effective diffusion range and the mean residence time of NTF2 in the channel.

Key to the observation of protein translocation through a NPC was a novel technique for NSOM imaging on unsupported membranes. For the manual preparation of such membranes, we used nuclei of unmature egg cells of an African claw frog (Xenopus laevis; stage VI oocytes) which have a typical diameter of $\sim 500 \mu \mathrm{m}$. After DiI-staining the NEs were spread on photolithographically fabricated templates exhibiting a regular grid of $20-\mu \mathrm{m}$-sized troughs (see figure $6.4 \mathrm{a}-\mathrm{b}$ ).
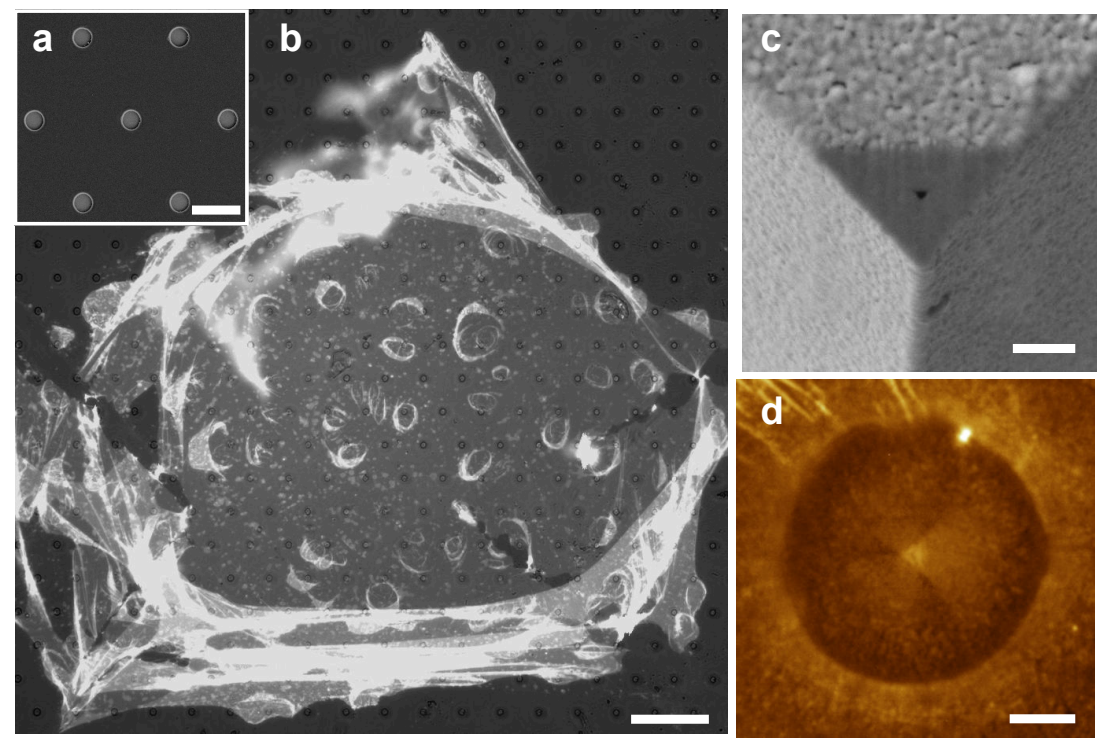

Figure 6.4.: Setup for NSOM imaging on unsupported membranes. (a, b) Manually prepared nuclear membranes were spread on a photo-lithographically fabricated template exhibiting a regular grid of $20-\mu \mathrm{m}$-sized troughs. The membrane was spanned over the troughs thereby establishing unsupported circular membrane patches of $20 \mu \mathrm{m}$ diameter. Scale bars, $50 \mu \mathrm{m}$ (a) and $200 \mu \mathrm{m}$ (b). (c) Electron micrograph of the near-field probe. Scale bar, $0,3 \mu \mathrm{m}$. (d) Near-field probe centered over a membrane patch. The typical side length of the triangular end face was $\sim 1 \mu \mathrm{m}$ rendering the probe well visible in an optical microscope. While scanning, the near-field probe was carefully lowered into the trough so that the membrane patch was set under a slight tension. The approach was stopped when no further increase of fluorescence intensity or optical resolution was observed. Scale bar, $5 \mu \mathrm{m}$ [89].

For NSOM imaging the near-field aperture probe was closely placed over a freely 
suspended membrane patch and carefully lowered into the trough until the membrane was set under a slight tension (see figure $6.4 \mathrm{~d}$ ). The probe-membrane distance was maintained without feedback control by using the elastic response of the nuclear membrane only. Even by scanning the probe across the NE in a constant-height mode with a scan speed of $>4 \mu$ s no distortion of the membrane could be observed and it appeared that the flat end face of the probe slid over an ultra-thin film of liquid. During imaging the membrane tension was fine-adjusted to further increase the fluorescence intensity and the optical resolution. However, typically the resolution could not be further improved, largely independent of the initial optical resolution. We assume that the absence of debris on the membrane surface after preparation is the most critical factor for achieving a close probe-sample distance and thus a high optical resolution.

Using such a set-up, we imaged the nucleoplasmic face of a freestanding native nuclear membrane stained with the Alexa Fluor ${ }^{\circledR} 633$ labeled pan-NPC antibody mAb414 (mAb414-A633) (see figure 6.5). The high optical resolution capability of NSOM and the extremely short depth of the evanescent field at the aperture enabled us to apply a steep intensity gradient along the axis of a single NPC. This offered the exciting prospect of being able to study the kinetics of protein translocation through an individual NPC. In the following, a one-dimensional diffusion model shall highlight the experimentally accessible quantities of a translocation through a pore using this approach.

\subsubsection{Analysis of complex diffusion mechanism in the light of the confined diffusion model}

Figure 6.6a schematically depicts a model for binding and diffusion of fluorescent particles moving in a channel with diffusion range $L$ that is illuminated along its axis by a laterally homogenous evanescent field of decay length $d$. In a first approach, the diffusion coefficient $D$ of the particles was assumed to be constant throughout the channel. Time traces of the fluorescence intensity were calculated using the simulation model of chapter 4 (see figure 6.6b). For the quantitative evaluation an FCS analysis was applied.

In the case of an infinite diffusion range $L$ and infinite residence time $T_{R}$ the theoretical ACF of the described situation is well-known and used, for example, to study the Brownian motion of molecules by means of total internal reflection microscopy (see section 4.1.2). Regarding the more general case of a confined diffusion (finite $L / d$ ) and finite residence time $T_{R}$ we derived an approximate analytic solution (see appendix A). Supposing that $T_{R}$ is much larger than the mean residence time of the particle in the evanescent field, $T_{d}=d^{2} / D$, the ACF resulting from the confined-diffusion model (CDM) is given by: 

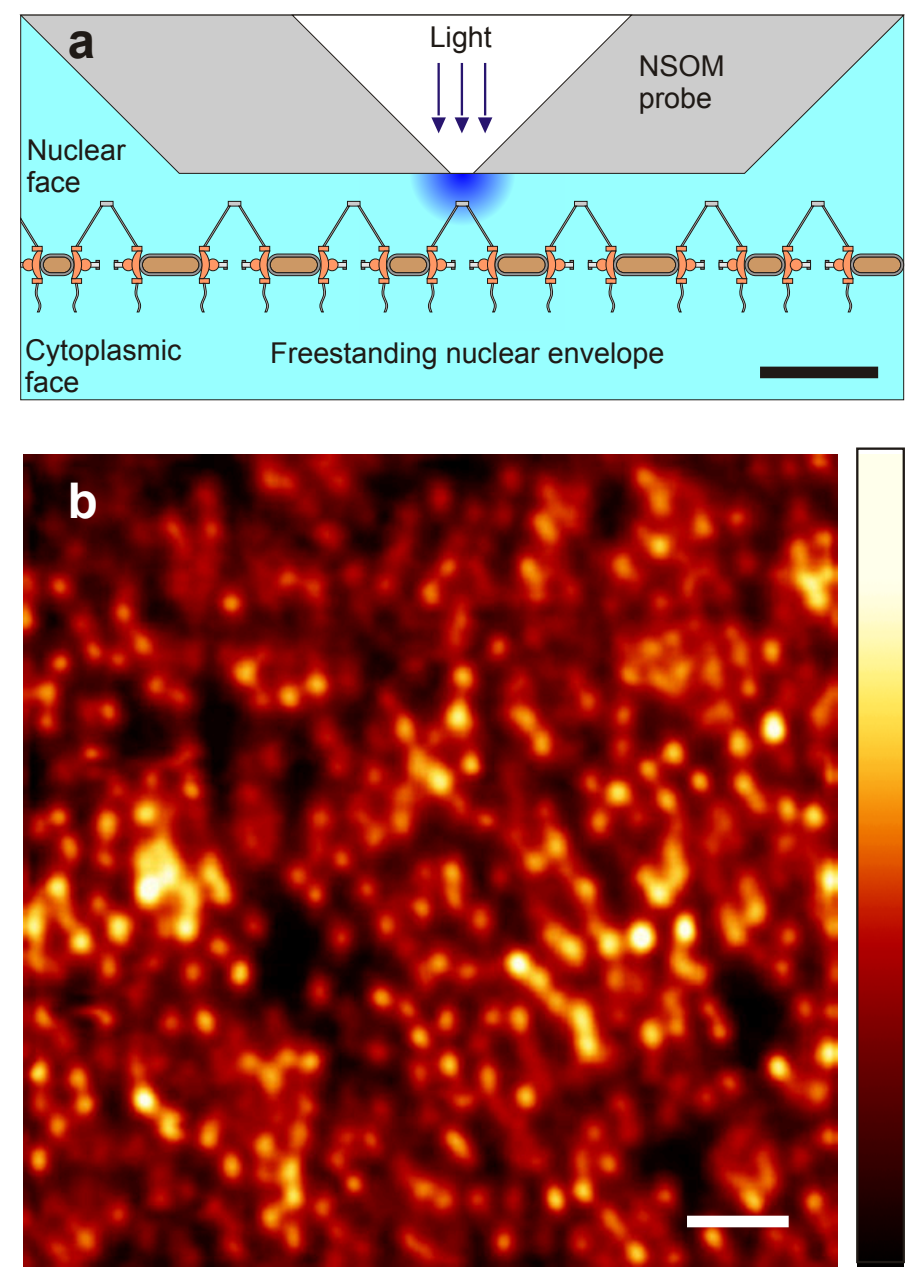

Figure 6.5.: NSOM imaging of a freely suspended native NE patch. (a) Schematic of a NSOM probe sliding over unsupported NE. Dependent on the pressure of the probe, the NPC baskets may extend up to $75 \mathrm{~nm}$ into the nuclear space and thus can act as a natural spacer between the light emitting aperture and the mid plane of the membrane. The evanescent field (blue) falls off exponentially with distance to the aperture on a scale of $10-30 \mathrm{~nm}$ so that chiefly fluorophores located at the nuclear side will be excited. Scale bar, $0.2 \mu \mathrm{m}$. (b) NSOM fluorescence image of the nucleoplasmic face of a freely suspended unfixed NE labeled with mAb414-A633. The size of the fluorescence spots of $60-90 \mathrm{~nm}$ agrees well with corresponding AFM measurements of the NPC basket. Scan speed, $4 \mu \mathrm{m} / \mathrm{s}$; intensity range, $0.25-3.0$ (arb. units); scale bar, $0.5 \mu \mathrm{m}$ [89]. 

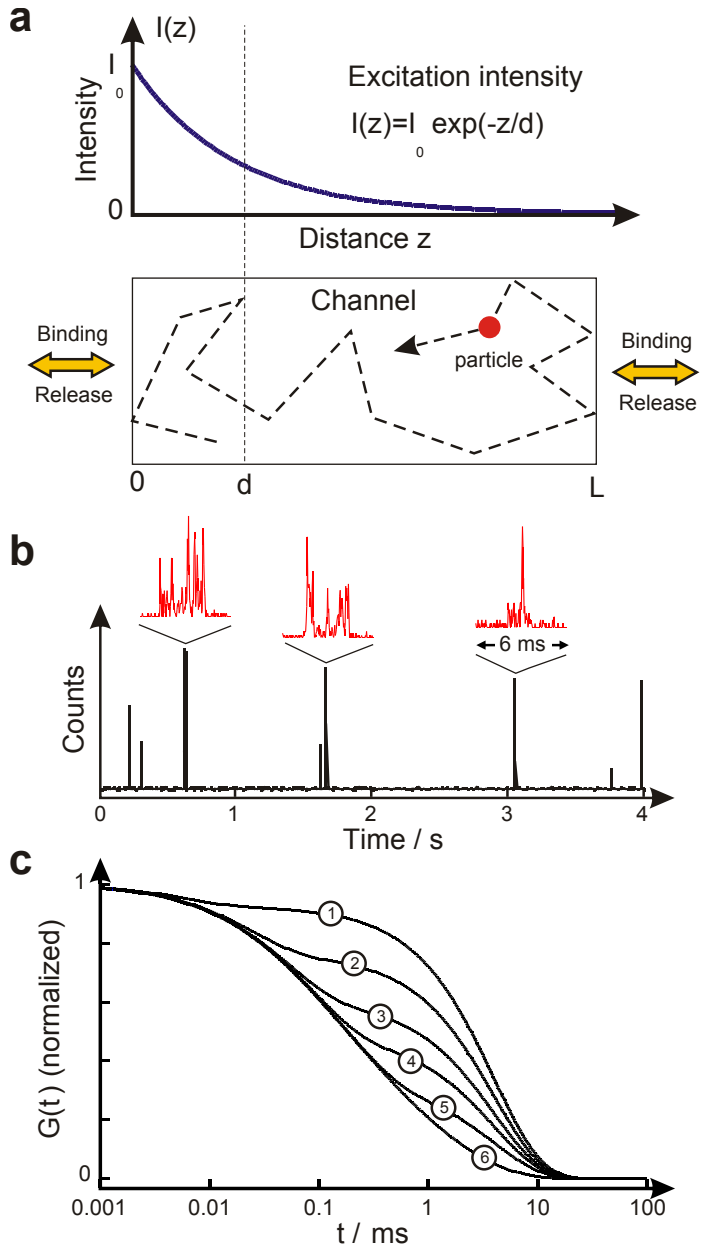

Figure 6.6.: Confined diffusion of fluorescent particles in an evanescent field. (a) A diffusion channel is illuminated along its axis by an evanescent field of depth d. A fluorescent particle with diffusion coefficient D is captured in the channel for a mean residence time TR, randomly moves back and forth within the diffusion range $\mathrm{L}$ and is fluorescently excited according to the local intensity $\mathrm{I}(\mathrm{z})$. (b) Time trace of the fluorescence intensity calculated by a Monte-Carlo simulation for $D=10 \mu \mathrm{m}^{2} / \mathrm{s}, L=100 \mathrm{~nm}$, $d=25 \mathrm{~nm}, T_{R}=4 \mathrm{~ms}$, and mean time between binding events of $T_{B}=1 \mathrm{~s}$ (black line). Representative fluorescence bursts accompanying capture and release of particles are displayed in enlarged views (red curves). (c) Normalized autocorrelation functions of the fluorescence fluctuations for different diffusion ranges L: 1) $25 \mathrm{~nm}, 2) 50 \mathrm{~nm}, 3$ ) $75 \mathrm{~nm}, 4) 100 \mathrm{~nm}, 5) 150 \mathrm{~nm}$, and 6) $\infty$ [89]. 


$$
\begin{aligned}
G_{c d}(\tau) \propto \frac{1}{\langle N\rangle}\left\{1+2 \sum_{m=1}^{\infty} \frac{\exp \left(-\left(\frac{m \pi}{L}\right)^{2} D \tau\right)}{\left(1+\left(\frac{m \pi d}{L}\right)^{2}\right)^{2}}\left(\frac{1-(-1)^{m} \exp \left(-\frac{L}{d}\right)}{1-\exp \left(-\frac{L}{d}\right)}\right)^{2}\right\} \times \\
\quad \exp \left(-\frac{\tau}{T_{R}}\right)
\end{aligned}
$$

with time lag $\tau$ and average number $\langle N\rangle$ of particles trapped in the channel. As an illustration, several autocorrelation curves for different values of $L / d$ are plotted in figure $6.6 \mathrm{c}$.

To clarify the effect of a locally varying diffusion coefficient along the axis of the channel, we carried out corresponding FCS simulations. The simulated ACFs turned out to have almost the same shape as $G_{c d}(\tau)$ and therefore can be excellently fitted by $G_{c d}(\tau)$ through a suitable choice of $L$ and $D$. Thus we found that the CDM is an adequate model even for the description of complex diffusional motion in a channel as long as the related parameters $D$ and $L$ resulting from a fit are considered as averaged over the real conditions. For the analysis of experimental data, we therefore regard $D$ as an apparent diffusion constant $D_{a p p}$ and $L$ as an effective diffusion range $L_{e f f}$. Assuming a decreasing diffusion coefficient toward the center of the pore, it turned out that $L_{\text {eff }}$ and $D_{a p p}$ represent minimum values for the real diffusion range and the real diffusion coefficient at the channel entrance, respectively.

\subsubsection{FCS measurement at a single nuclear pore}

For a NSOM-FCS measurement, NTF2-A633 was added to the buffer solution covering a freshly prepared NE and the NSOM probe was placed over a single pore to collect the fluorescence intensity $F$ as a function of time (see figure 6.7a). The appearance of intense fluorescence bursts of some milliseconds clearly indicated binding of NTF2-A633 to the NPC with a typical residence time of $T_{R} \sim 1-5 \mathrm{~ms}$ (see figure 6.7b). On a much shorter time scale (time bin $25 \mu \mathrm{s}$ ) these bursts turned out to be composed of several peaks of varying intensity at irregular intervals (see figure $6.7 \mathrm{~b}$, red curves). The typical length of the short events and hence the mean residence time $T_{d}$ of NTF2-A633 in the evanescent field of depth $d \approx 25 \mathrm{~nm}$ was in the order of $25 \mu \mathrm{s}$ only. We interpret such a continuing series of short events as intermittent fluorescence of a single NTF2 that was randomly pushed into the evanescent field at the channel entrance while caught in the transport channel. Our observations suggest that bound NTF2 proteins are not restricted to the central region of the NPCs but have a high degree of mobility along the pore axis.

To get a precise measure of the mobility of NTF2-A633 within the NPC we evaluated the autocorrelation function $G_{\text {pore }}(\tau)$ of the respective fluorescence data (see figure $6.7 \mathrm{c}$, green line). In principal, $G_{\text {pore }}(\tau)$ comprises the ACF of NTF2A633 binding to the pore, $G_{\text {bound }}(\tau)$, as well as the ACF of NTF2-A633 diffusing 
a

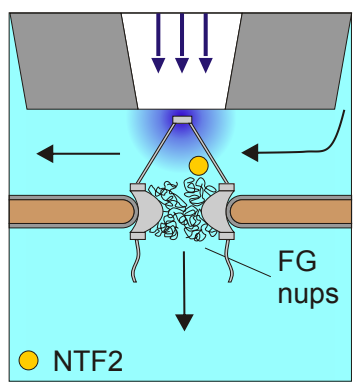

C

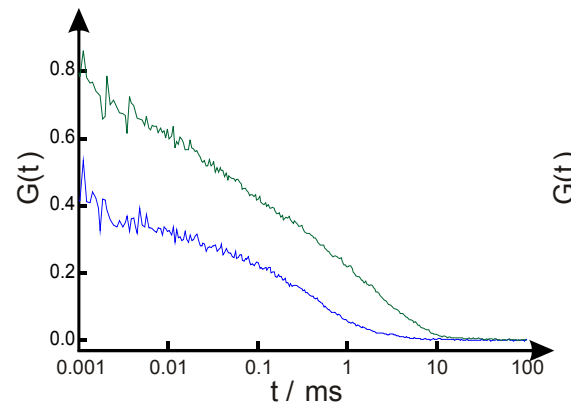

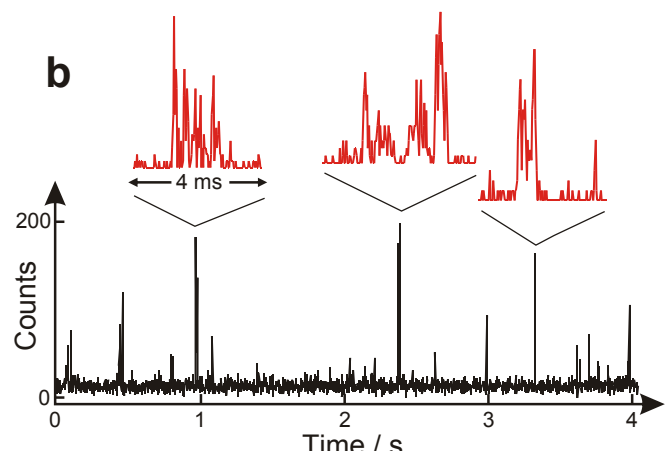

d

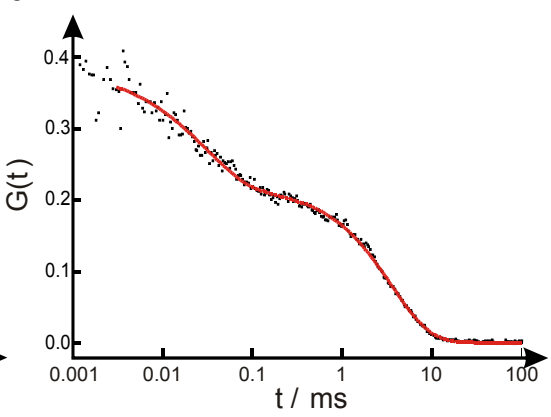

Figure 6.7.: Diffusional motion of NTF2 within NPCs observed by NSOM-FCS. (a) Schematic of the experiment: the NSOM probe is placed over a pore and the fluorescence of NTF2-A633 is measured as a function of time. (b) Typical time trace of NTF2-A633 fluorescence for a measurement over a pore (black line, time bin $1.6 \mathrm{~ms}$ ). On a much shorter scale (time bin $25 \mu \mathrm{s}$ ) the bursts are composed of several peaks of varying intensity (red curves). (c) ACF of a time trace measured over a pore (green line). To determine the background, FCS curves were collected at a location beside a pore (blue line). (d) Pore ACF after subtracting the background $b \cdot G_{b g}(t)$ (dots) and related least-squares fit (red line) $(a=0.71 \pm 0.02, b=0.88 \pm 0.03$; see text). The fit is based on the confined-diffusion model with fixed $d=25 \mathrm{~nm}$. Averaging over several measurements, we obtained $L_{e f f}=85 \pm 11 \mathrm{~nm}, D_{a p p}=17 \pm 3 \mu \mathrm{m}^{2} / \mathrm{s}$, and $T_{R}=3.6 \pm 0.5 \mathrm{~ms}$. [89]. 
in the solution between NSOM tip and NE, $G_{s o l}(\tau)$. Since the residence time of NTF2 at the pore is much longer than the residence time in the evanescent field, $T_{R} \gg T_{d}, G_{\text {pore }}(\tau)$ can be expressed as a weighted sum of both contributions, $G_{\text {pore }}=A \cdot G_{\text {bound }}(\tau)+B \cdot G_{\text {sol }}(\tau)$ [73]. The factors $A$ and $B$ depend on the concentration of molecules, the equilibrium constant of the reaction, and the average numbers of observed molecules in solution and at the binding site. While NTF2 is bound to the pore, it will only be excited by the evanescent field when it is located near the entrance of the transport channel. Assuming that the channel is homogeneously illuminated in lateral direction and that NTF2 is traveling up and down the channel in a random walk, we can approximate $G_{\text {bound }}(\tau)$ by the ACF of the CDM, $G_{c d}(\tau)$. For an independent measure of $G_{s o l}(\tau)$, we determined the fluorescence fluctuations caused by lateral diffusion alone at a location beside a nuclear pore. The corresponding "background" correlation function $G_{b g}(\tau)$ is displayed in figure 6.7c (blue line). A comparison of background and pore ACF clearly shows that the pore $\mathrm{ACF}$ is much more pronounced at time lags $>1 \mathrm{~ms}$ which likely reflects the binding of NTF2 to the pore. Supposing that the background ACF is almost constant across the NE, we can equate $G_{b g}(\tau)$ with $G_{s o l}(\tau)$ and finally arrive at $G(\tau)=a \cdot G_{c d}(\tau)+b \cdot G_{b g}(\tau)$ as a fit function for the measured pore ACF $G_{\text {pore }}(\tau)$.

The resulting least-squares fit (see figure $6.7 \mathrm{~d}$ ) shows a good correspondence to the data. Averaging over several measurements, we determined the apparent diffusion coefficient to $D_{a p p}=17 \pm 3 \mu \mathrm{m}^{2} / \mathrm{s}$ and the residence time in the transport channel to $T_{R}=3.6 \pm 0.5 \mathrm{~ms}$. The resulting effective diffusion range $L_{\text {eff }}=$ $85 \pm 11 \mathrm{~nm}$ agrees well with the length of the transport channel of $\sim 90 \mathrm{~nm}$ as found in a 3D reconstruction of the NPC based on cryo-electron tomography [93]. Assuming that the length of the transport channel is smaller than $90 \mathrm{~nm}$, the large value for $L_{\text {eff }}$ may be interpreted as indication for a temporary binding of NTF2 to FG-repeat nups reaching out beyond the central framework of the NPC. Recent NPC models suggest an increasing density of FG-repeat nups toward the center of the pore and thus imply a varying diffusion coefficient along the channel axis. As discussed above, the found numbers for $L_{e f f}$ and $D_{a p p}$ therefore represent minimum values for the real diffusion range and the real diffusion coefficient at the channel entrance.

In summary, we solved a major obstacle of NSOM imaging on soft matter in solution by establishing a novel distance control based on the weak elastic response of a freely suspended membrane patch. This "passive" distance control enabled us to reliably image a native NE at high optical resolution. As the transport channel of the NPCs was no longer obstructed by a support, the translocation of proteins through a single pore could be studied on a microsecond time scale by placing the near-field probe over a pore. The extremely short depth of the evanescent field in axial direction of $\sim 25 \mathrm{~nm}$ generated a steep intensity gradient along the axis of the transport channel, so that labeled proteins, NTF2-A633, produced strong fluorescence fluctuations by traveling up and down the channel. These fluctuations were evaluated by means of a fluorescence correlation analysis based on a model of Brownian motion in a confined space. In that way, the features of NSOM, ultra-high 
spatial and temporal resolution, were employed to reveal the kinetics of a transport process on a single-molecule level. 



\section{Detailed characterization of near-fields at an aperture probe with FCS}

Following the fabrication process explained in the last chapter, numerous triangular aperture (TA) probes with high quality of the triangular shape could be obtained. This chapter presents a selection of the numerous high-quality FCS measurements that have been carried out with these probes. This quality is mainly shown by the low variance of the FCS curves at lag times as small as the resolution limit of the experimental setup, i.e. $1 \mathrm{~ns}$, which requires measurements with a high signal-tonoise ratio (SNR). This SNR is evaluated in section 7.2. Field enhancement effects similar to these occuring at nanoantennas are considered to explain this high SNR. The very small excitation volume, which was evaluated to be 3 attoliters for the triangular aperture probe model of section 4.2.3, is confirmed in section 7.3 with NSOM-FCS measurements with molecular concentrations up to $25 \mu \mathrm{M}$. Also the same high time resolution of NSOM-FCS with TA probes was confirmed (section 7.4). Finally, it will be shown in section 7.5 that a simple free diffusion model cannot account for some measurements that show fluctuation at large lag times depending on the solvent characteristics. But first, we will start this chapter with an evaluation of the reproducibility of NSOM-FCS measurements.

\subsection{Experimental aspects of NSOM-FCS measurements}

When considering a measurement technique, it is important to know how stable and how reproducible the measurements are. The stability of a measurement is related to how much the measurement results vary when keeping the same measurement parameters and measuring over a long time, whereas its reproducibility indicates to what extent this measurement with a given set of parameters can be reproduced at a later time with the same set of parameters. In this work and with the fabrication technique for the TA probe, the reproducibility is widely ensured if the same TA probe is used from one measurement to the next with otherwise same experimental conditions. These other experimental conditions are essentially the laser intensity, the concentration of fluorescent molecules, the solvent, the optical setup alignment and the probe size. In the case where reproducibility is evaluated by considering 
the probe size ${ }^{1}$ instead of keeping the same probe, a mismatch can be observed between two test measurements.

\subsubsection{Stability of FCS measurements with a TA probe}

The several NSOM-FCS measurements carried out during this thesis proved to be quite stable as shown, for instance, in figure 7.1. In this measurement series, the cross correlation of the fluorescence signal at the two detectors was taken approximately every minute and a minute long. The cross correlation functions show a very similar form, which is a good indicator that the measurements are stable over a long period of time. This is a condition for measurements of slow dynamic processes with typical diffusion times of 10 to $100 \mathrm{~ms}$. To obtain a good statistical accuracy, the acquisition time should be around 10,000 times longer than the diffusion time of the measured process [98]. The time trace of one of the measurements of figure 7.1 is shown in figure 7.2 over a period of $60 \mathrm{~s}$ and with a time resolution of $1 \mathrm{~s}$. The constant value of the fluorescence signal in this time trace is an additional evidence for the stability of the measurement: no photophysical destruction of the fluorescent molecules or vaporization of the solvent leading to a higher fluorescent signal can be detected.

Eventually, after longer waiting time between two measurements the increase of the molecular concentration in solution begins to be visible: in figure 7.3, 20 minutes elapsed between the two measurements. As a result of the partly vaporization of the solution, its molecular concentration increases and the mean number of molecules $N$ in the excitation volume at the aperture increases. Since the amplitude of the correlation function is inversely proportional to $N$ (see for example equation 3.55), this amplitude decreases. Additionally, the fluorescence signal detected increases proportionally to $N$. This is also reflected by the detected count rates for these two measurements. It has to be noted however that an additional readjustment of the light coupling in the near-field probe had to be undertaken before the second measurement. This is probably due to mechanical constraints in the excitation setup, particularly in the setup components being translated by a system of screws and springs (see figure 5.7)

\subsubsection{Reproducibility of NSOM-FCS measurements}

The reproducibility of NSOM-FCS can be evaluated by carrying out two measurements with the same parameters such as optical setup, laser intensity and molecular concentration but by disturbing the alignement of the optical setup, changing temporary the laser intensity, taking a fresh coverslip and depositing the same amount of another batch of the solution of fluorescent molecules with the same concentration and then trying to achieve the same results as in the first measurement.

\footnotetext{
${ }^{1}$ The probe size in this chapter is evaluated as the height of the triangular aperture using electron micrographs such as in figure 5.6.
} 


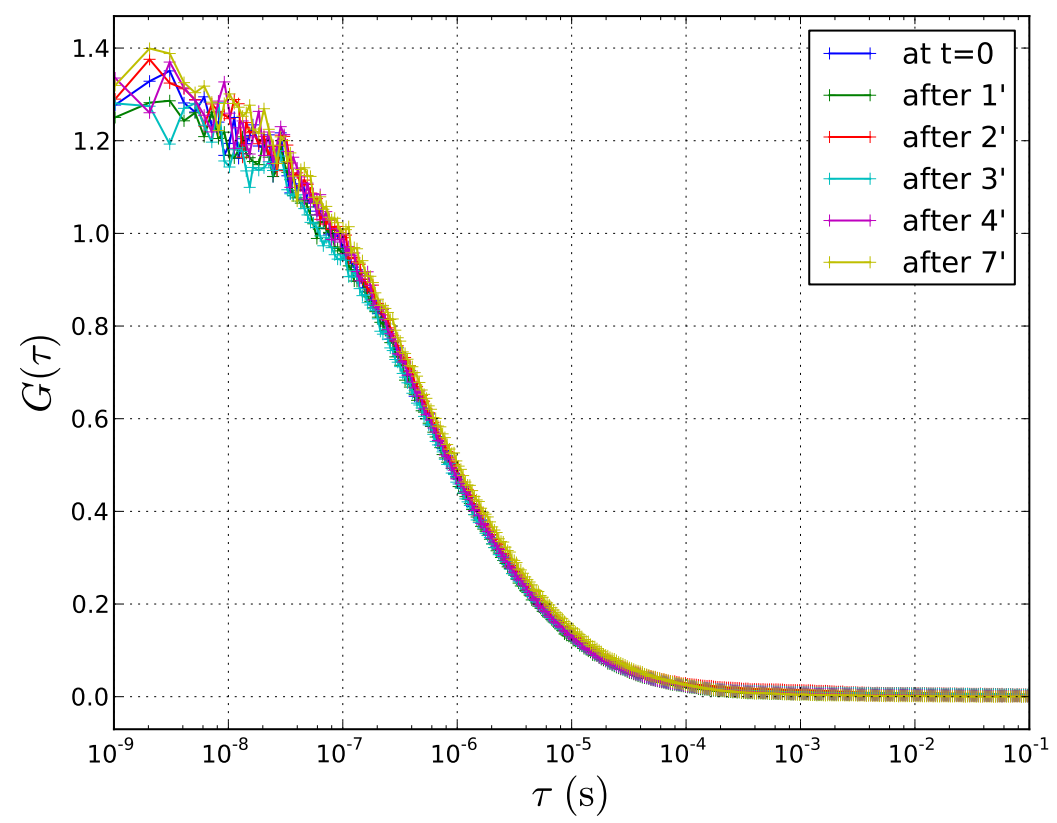

Figure 7.1.: Stability of the cross correlation of the fluorescence signal for a series of FCS measurements with no change in experimental conditions. The five measurements were taken one minute long and approximately every minute with an aperture probe, which aperture size is about $135 \mathrm{~nm}$. The fluorescent molecules are Atto655 in concentration $250 \mathrm{nM}$ in phosphate buffer saline with $0.5 \%$ of the detergent Tween20 (PBST). Averaging the count rate over these five measurements leads to a mean count rate of about $261 \pm 8 \mathrm{kHz}$. 


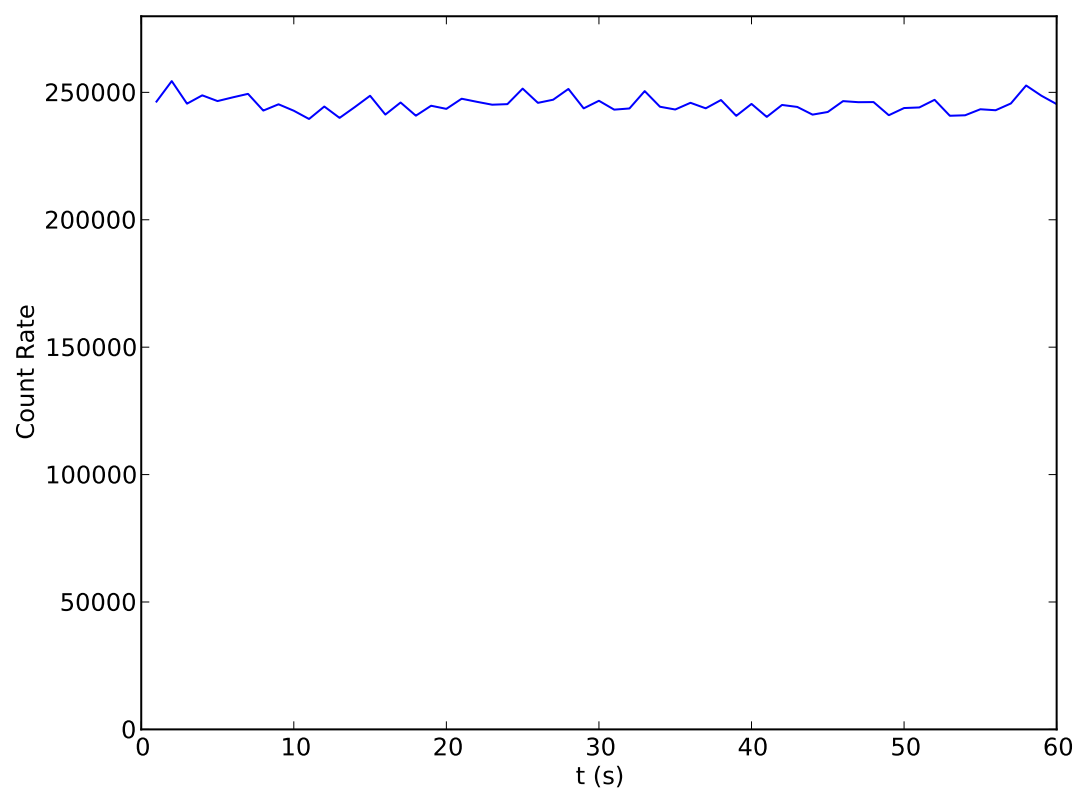

Figure 7.2.: The time trace of one of the measurements presented in figure 7.1. The stability of the fluorescence signal indicates that neither the fluorescent molecules suffer photophysical destruction (which would lead to a decrease of the fluorescence signal) nor that the vaporization of the solution is high (which would lead to a more concentrated solution in fluorescent molecules and consequently to a higher fluorescence signal). 


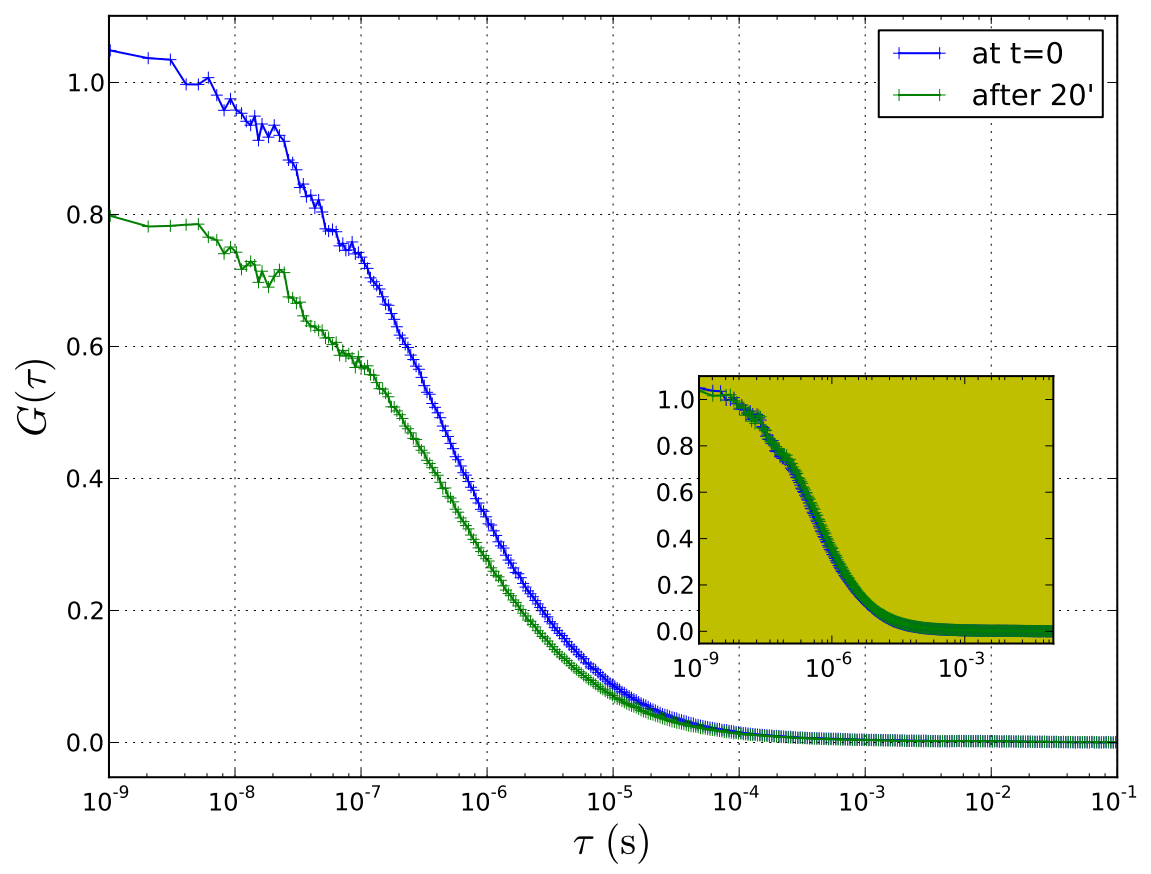

Figure 7.3.: Result of a longer waiting time between two FCS measurements in otherwise the same experimental conditions. These two measurements were taken with 20 minutes time difference, with a probe of aperture size of about $60 \mathrm{~nm}$. The fluorescent molecules are Atto655 with $100 \mathrm{nM}$ concentration in phosphate buffer saline (PBS). The amplitude ratio between the two measurements is about 1.3 , compatible with the mean count rate increase of about 554 to $748 \mathrm{kHz}$. The inset shows the normalized measurements. 
An example is given in figure 7.4, which shows two series of measurements taken one after another following the procedure described before. It can be seen that the amplitude of the cross-correlation function increases between the two series of measurements (relative increase of about 1.7). Additionally the signal count rate at the detectors decreases (relative decrease of 1.9). The variation of these two measurement values in opposite direction suggests that only the mean number $N$ of molecules in the excitation volume changed between the two series of measurements. This is compatible with the proportionality relation between $N$ and the amplitude $G(0)$ of the cross-correlation function: $G(0)=1 / N$ (see equation 3.55).

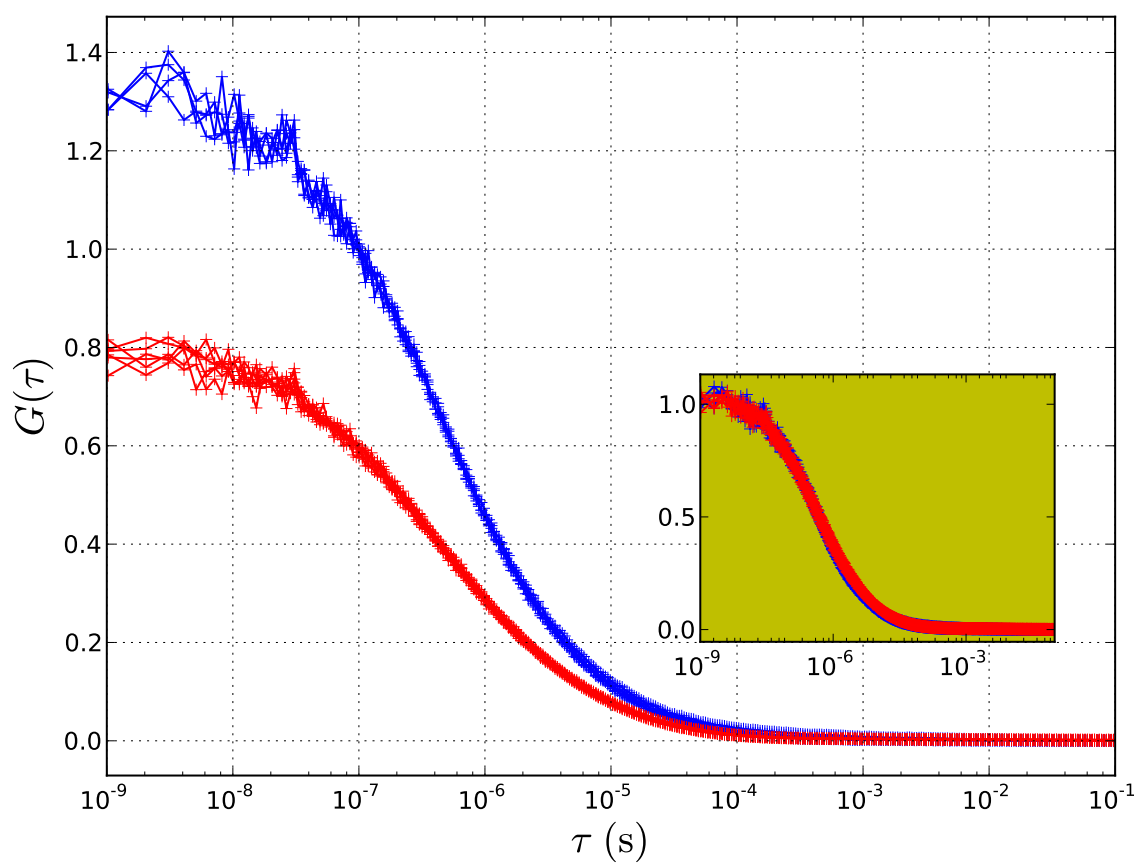

Figure 7.4.: FCS measurements illustrating the reproducibility of the measurements with a probe of size $\sim 135 \mathrm{~nm}$. The blue curves represent FCS measurements that were taken at a later time than the FCS measurements represented by the red curves with a different batch of the solution of Atto655 but with the same concentration $(250 \mathrm{nM})$. The amplitude of the FCS measurements at a later time (blue) increased by a factor of $\sim 1.67$, whereas the mean count rate $(220 \pm 2 \mathrm{kHz}$ for the blue curve and $415 \pm 6 \mathrm{kHz}$ for the red curve) decreased by a factor of $\sim 1.89$. However the course of the cross-correlation curve is conserved in the two cases as can be deduced from the perfect superposition of the normalized curves (inset). The power at the back aperture of the objective focusing the light in the aperture probe was $1.9 \mathrm{~mW}$.

A possible reason for the deviations between the two measurements might be slightly different effective concentrations due to pipetting errors. But also other 
reasons not as straightforward can be invoked: the fluorescent molecules can be partly adsorbed to the glass surface of the coverslip or to the large gold surface of the probe. The adsorption can vary depending on the coverslip but also on the measurement history of the probe: if it was previously used with a solution containing molecules that already adsorbed on the gold surface, this surface has different adsorption characteristics for the next measurement. This dependence on previous measurements can be partly removed by dipping the probe in pure water for some minutes. A more aggressive cleaning method using sonication should be avoided since it damages the probe at the aperture and consequently results in completely different FCS measurements. Also differences in the optical setup can lead to deviation in the measurements: variations in the thickness of the coverslip (thickness given with a precision of $\pm 5 \mu \mathrm{m}$ ), the surface of which is used to position the tip of the probe axially (position precision estimated to $1 \mu \mathrm{m}$ ) using a widefield image of the probe through an objective with a $100 \times$ magnification can add variation in the light path from the tip to the objective. This consequently leads to other values of the intensity of the fluorescence signals as well as small distortion in the measured excitation volume.

Another example of reproducible FCS measurements with the TA probe is given for a much smaller aperture size of $\sim 30 \mathrm{~nm}$ in figure 7.5 . Due to the greater variance of the cross-correlation functions, the averaged FCS measurements are shown instead of the single ones. In this case, the mean count rates and the amplitudes of the cross-correlation functions are slightly different but their evolution is compatible with the fact that these two dimensions are inversely proportional to each other. However, the proportionality relation has a larger deviation from one measurement to the other compared with the probe of larger aperture taken for the measurements of figure 7.4.

These measurement examples show that, if the reproducibility of the qualitative course of the cross-correlation function is widely ensured, the determination of the mean number of molecules in the excitation volume from the amplitude of this function should be done carefully.

\subsubsection{Variability of NSOM-FCS with different TA probes}

When the light intensity coupled into the TA probe is changed between two measurements with the same parameters, the reproducibility of NSOM-FCS can be impaired and variability in the measurements introduced. High intensities at the tip of some probes may be responsible for structural changes at the aperture with an accompanying change in the intensity distribution. But it is not totally clear which conditions (aperture size, metal coating quality) favor these changes. In figure 7.6, this fact is shown, for instance, with a probe with a rather large aperture: $\sim 95 \mathrm{~nm}$. Not only is the course of the cross-correlation function changed, but also its amplitude $G(0)$ and the mean number of photons detected do not behave as would be expected from the relation in equation 3.55, if the mean number of photons is proportional to the mean number of molecules $N$ in the excitation volume. 


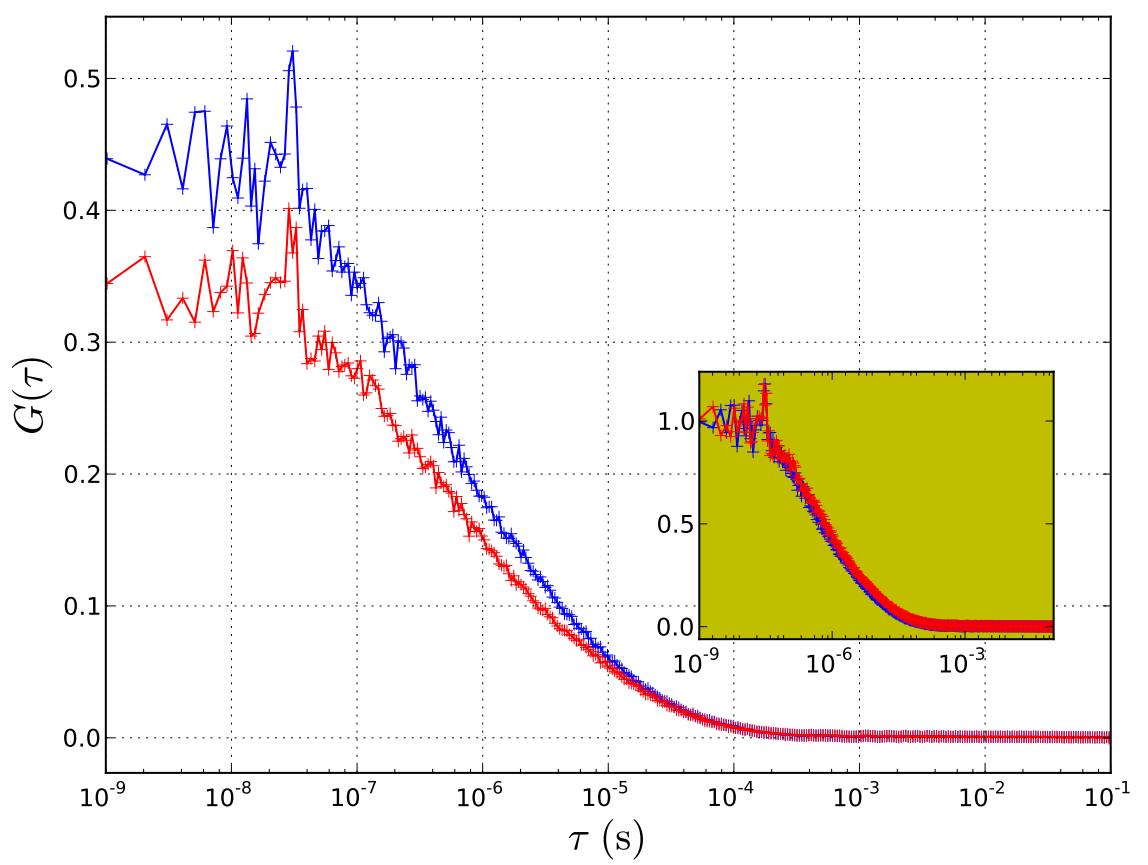

Figure 7.5.: FCS measurements illustrating the reproducibility of the measurements with a probe of size $\sim 30 \mathrm{~nm}$. The red curve represents the average of two FCS measurements that were taken at a later time than the five FCS measurements, the average of which is represented by the blue curve. The solution of Atto655 was from a different batch but with the same concentration $(250 \mathrm{nM})$. The amplitude of the FCS measurements at the later time (red) decreased by a factor of $\sim 1.29$, whereas the mean count rate $(109 \pm 8 \mathrm{kHz}$ for the blue curve and $199 \pm 4 \mathrm{kHz}$ for the red curve) increased by a factor of $\sim 1.8$. The power at the back aperture of the objective focussing the light in the aperture probe was $\sim 500 \mu \mathrm{W}$. The peak at $\sim 30 \mathrm{~ns}$ is an artificial correlation due to the detection setup (see section 5.2.2). 


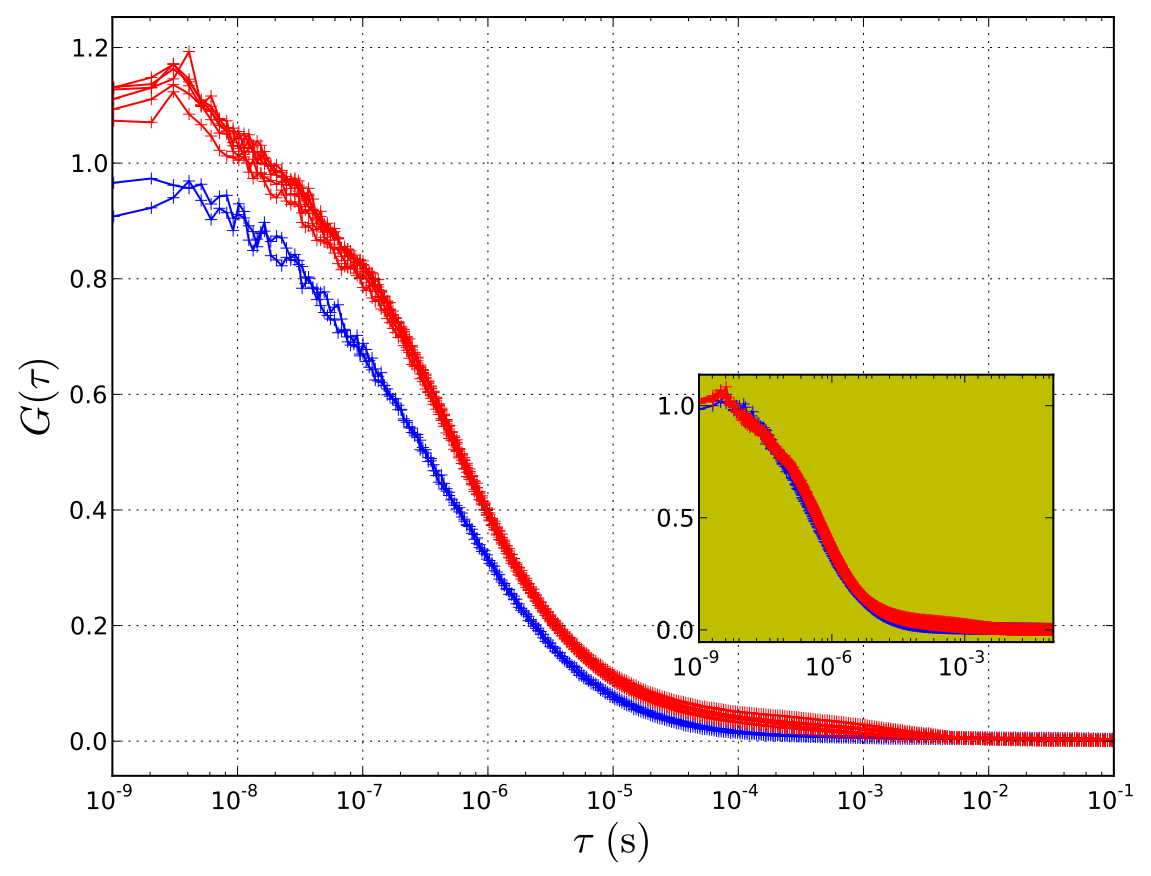

Figure 7.6.: FCS measurements illustrating the influence of the light intensity coupled into the probe on the reproducibility of the measurements with a probe of size $\sim 95 \mathrm{~nm}$. The red curves represent FCS measurements that were taken at a later time than the FCS measurements represented by the blue curves with a different batch of the solution of Atto655 but with the same concentration $(250 \mathrm{nM})$. The amplitude of the FCS measurements at the later time (red) increased with a factor of $\sim 1.16$ and also the mean count rate $(400 \pm 1 \mathrm{kHz}$ for the blue curve and $840 \pm 20 \mathrm{kHz}$ for the red curve) increased by a factor of $\sim 2.1$. The power at the back aperture of the focusing objective was $1.9 \mathrm{~mW}$. The course of the cross-correlation curve is also slightly modified as can be deduced from the perfect superposition of the normalized curves (inset). 
For the FCS measurements of figure 7.6, the modification of the aperture and its surrounding coating and the intensity distribution around it, seem to be positive regarding the count rate per molecules, the key parameter to obtain good SNR in FCS [20], [17]. If no other fluctuation mechanism as resulting from the free diffusion process of the fluorescent molecules is taken into account, then the count rate per molecule $(\mathrm{CRM})$ is simply $\mathrm{CRM}=G(0) \cdot \mathrm{CR}$, where $\mathrm{CR}$ is the count rate of the detected photons. This value is more than doubled between the two measurements, which also means that the FCS measurement can be carried out with a less than four times smaller duration to obtain the same statistical accuracy [20]. However, it is hard to say if these modifications are always beneficial for the FCS measurements.

The difficulty to obtain quantitatively reproducible measurements in this case may be due to the complexity of the interaction between light and the probe and its intensity dependent structural changes. Another example of the complexity of this interaction can be found by observing the difference in amplitude of the cross-correlation and the count rates between the FCS measurements with probes of growing aperture size: $30 \mathrm{~nm}$ (figure 7.5), $95 \mathrm{~nm}$ (figure 7.6) and $135 \mathrm{~nm}$ (figure 7.4). The attempt to scale these measured values with the probe size is very hard. This is because another fluctuating source disturbs the measurement: uncorrelated background noise stemming from the gold coating. Its effect is discussed in the next section.

\subsection{Insight into the signal-to-noise ratio of NSOM-FCS}

It has been shown in the previous section that for different aperture sizes, the FCS measurements do not reflect the expected scaling between excitation volume and aperture size. This observation results from the consideration of the amplitude of the cross-correlation function, which should increase with decreasing aperture size if only fluctuation arising from diffusional processes is taken into account. This section deals with the reasons for this absence of scaling: background signal and fluorescence enhancement introduced by the TA probe.

\subsubsection{Background origin of NSOM-FCS using gold coated TA probes}

Measurements of the signal detected when an aperture probe is dipped into a solution without fluorescent molecules show that the background noise has an effect on the measurements, which is far from being negligible. The general effect of uncorrelated background noise was discussed in section 3.2.2. It does not disturb the course of the cross-correlation function since it does not introduce additional correlation, but leads to a decrease of its amplitude corresponding to equation 3.59. This 
background noise is attributed to electronic transitions in the gold coating. There are two possible kinds of electronic transitions that can be taken into consideration. On the one hand, the best known photoluminescence mechanism in gold is the interband transition mechanism between the $s p$ conduction band and the lower lying $d$ bands. This transition mechanism has been reported in [99]. The corresponding emission shows a peak at about $560 \mathrm{~nm}(2.2 \mathrm{eV})$ with an excitation wavelength up to $514.5 \mathrm{~nm}$. Using a lower energetic excitation wavelength of $640 \mathrm{~nm}$, the photoluminescence of gold due to interband transitions is not expected to be very high but still emission in the wavelength range $685 \pm 20 \mathrm{~nm}$ is possible (other wavelengths are rejected by the combination of filters before detection). Another transition mechanism that was introduced to explain the broad visible and infrared spectrum detected at gold nanostructures is an intraband transition mechanism [100]. In the electric dipole approximation, this kind of transition is forbidden because the initial and the final state have the same symmetry. Furthermore the additional momentum needed to make the transition cannot be mediated by a propagating photon. However, in the vicinity of gold nanostructures such as gold nanospheres or simply roughnesses in a gold film, the high field gradients make the dipole approximation unsound and higher-order transition moments have to be taken into account, for which the transitions between electronic states of same symmetry may very well be allowed. Additionally, the electric near-field at those nanostructures convey a large spectrum of spatial frequencies (see section 2.1.1), from which the necessary additional momentum to make the electronic transition can be mediated.

This photoluminescence of the gold film that is responsible for the emission of uncorrelated noise, gives a viable explanation for the absence of scaling of the different count rates and amplitude of FCS measurements in figures 7.5, 7.6 and 7.4 with the size of the probe. A difference in the coating quality of the different probes could be responsible for a different intensity of the noise in each measurement. Even if the gold surface around the aperture should be very flat, as it was milled using a focused ion beam, the gold film cannot be perfectly homogeneous. This is due to the sputtering process, through which gold clusters cover the glass surface leading to a grain structure in the coating visible in the electron micrographs of the aperture probe (see for example 5.6). Since the probes used for the mentioned FCS measurements were coated together, coating conditions cannot be taken responsible for a difference in the coating quality. The roughness of the glass surface at the tip may be responsible for these differences. An additional possibility is a sort of distance dependent roughness of the coating to the apex. The coating of the three probes involved were capped at different heights to get an aperture of different size. The grain structure might be different at the very end of the apex as a few nanometers further away, leading to another noise emission characteristic of the gold film. 


\begin{tabular}{|c|c|c|c|}
\hline Parameters & $30 \mathrm{~nm}$ probe & $95 \mathrm{~nm}$ probe & $135 \mathrm{~nm}$ probe \\
\hline Background CR $(\mathrm{kHz})$ & $\sim 13$ & $\sim 340$ & $\sim 25$ \\
Total CR $(\mathrm{kHz})$ & $\sim 195$ & $\sim 689$ & $\sim 229$ \\
Uncorrected $G(0)$ & 0.34 & 1.33 & 1.17 \\
Corrected $G(0)$ & 0.39 & 5.18 & 1.48 \\
CRM $(\mathrm{kHz})$ & $\sim 71$ & $\sim 1.8 \cdot 10^{3}$ & $\sim 302$ \\
\hline
\end{tabular}

Table 7.1.: The parameters used to evaluate the influence of the gold photoluminescence on the FCS measurements of three probes of different aperture sizes. CR stands for count rate and "Background CR" corresponds to a measurement without fluorescence molecules, whereas "Total CR" represents the measurement with fluorescence molecules, i.e. a measurement where the signal detected is the superposition of the fluorescence signal from the molecules and the background due to the photoluminescence of gold. The value of the cross-correlation function at zero time lag $G(0)$ is used to identify the mean number of molecules in the excitation volume in the case of noisy measurements (see equation 3.59). The "Uncorrected $G(0)$ " value is therefore the value of the cross-correlation function as obtained from the curve, whereas "Corrected $G(0)$ " is the same value divided by the factor $\left(1-I_{\mathrm{B}} / I_{\mathrm{TOT}}\right)^{2}$, where $I_{\mathrm{B}}$ is proportional to the "Background CR" value and $I_{\mathrm{TOT}}$ to the "Total CR" with the same proportionality constant. Finally, the mean count rate per molecules (CRM) is computed by substracting the background count rate to the total count rate and multiplying by the corrected $G(0)$ value.

\subsubsection{Probe aperture size dependent emission of background noise}

In order to quantify the influence of the probe aperture size on the background noise, the background signal in absence of fluorescent molecules in solution and the total signal in presence of these molecules were measured for the three different probes used for the measurements given in figures 7.5, 7.6 and 7.4 and for a definite power of $500 \mu \mathrm{W}$ at the back focal plane of the focusing objective (see figure 5.7). The different measured count rates are given in table 7.2.2. The FCS measurements with the fluorescent molecules are given in figure 7.7 and the amplitude of the crosscorrelation functions at zero lag time are also listed in table 7.2.2. Together these different values allow one to compute the "true" fluorescence signal count rate, i.e. that coming from the molecules and finally to derive a mean count rate per molecules. The resulting values are also given in 7.2.2.

Considering the values of the different cross-correlations at zero lag time corrected for the background ("Corrected $G(0)$ "), a correct behavior can be assumed between 


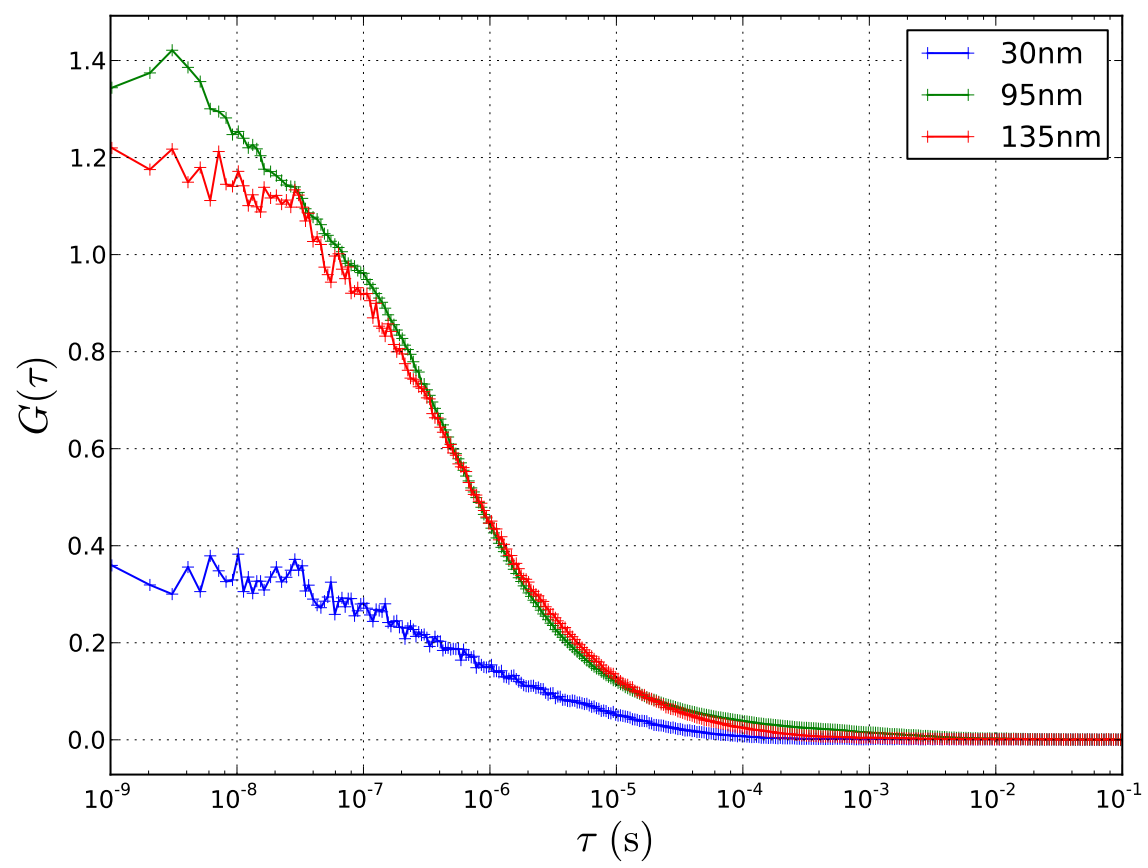

Figure 7.7.: NSOM-FCS measurements for different probe sizes. In the three cases the concentration of Atto655 and the power at the back focal plane at the focusing objective were kept to $250 \mathrm{nM}$ and about $500 \mu \mathrm{W}$, respectively. 
the values for the large aperture probe $(135 \mathrm{~nm})$ and the medium sized aperture probe $(95 \mathrm{~nm})$, for which the corrected $G(0)$ value is compatible with the decrease in the excitation volume for the smaller aperture (see equation 3.55: $G(0)=1 / N$ and $N=C_{0} V_{\text {exc }}$ from section 3.2.2). It could be expected, however, that the excitation volume is reduced of a factor corresponding to the aperture ratio in the three directions $x, y$ and $z$. Consequently, the mean number $N$ of molecules in the excitation volume should scale with the third power of the aperture ratio between the two probes. The observed increase of the corrected $G(0)$ value exceeds this expected value. For the probe of smallest aperture size, the scaling behavior is not reproduced at all, even qualitatively. Furthermore the count rates per molecule are not constant at all between the three probes. Consequently, taking the background into account is not sufficient to explain why the FCS measurements do not reflect a proportionality between aperture size and excitation volume. An influence of triplet occupation of the molecules on the amplitude of the corrected $G(0)$ is considered very unlikely since the fluorescent molecule used for these experiments, i.e. Atto655, is known to show nearly no triplet excitation [70].

\subsubsection{Antenna effects at the triangular probe}

A possible effect that could account for size dependent results in the FCS measurements is the antenna effect that was mentionned in section 2.2.2. In such a case, the fluorescent molecules are not only excited by a strong near-field intensity but their fluorescence emission is also amplified. This amplification is also strongly localized at the nanoantenna such that a seemingly higher confined field excites the molecules. Consequently this would also lead to a seemingly smaller excitation volume in the FCS measurements. This could explain the high corrected $G(0)$ value for the $95 \mathrm{~nm}$ large aperture probe. Such an enhancement could also explain the very high count rate per molecules measured for this probe (see table 7.2.2). Additionally, if an enhancement of the fluorescence emission takes place, then a decrease of the fluorescence lifetime should be observed. To test the hypothesis of fluorescence enhancement at the probe, the fluorescence lifetime of Atto655 was measured both at the probe and in a confocal experiment. The resulting measurements are shown in figure 7.8 .

The measured fluorescence signal $F_{C}(t)$ is given by the convolution of the instrument response function (IRF) with the fluorescence lifetime $F(t)$ of the fluorescent molecule:

$$
F_{C}(t)=\int_{0}^{\infty} \operatorname{IRF}\left(t^{\prime}-t_{0}\right) F\left(t-t^{\prime}\right) \mathrm{d} t^{\prime}
$$

The constant offset $t_{0}$ ensures best fitting results. Additionally, following expression for $F(t)$ were used:

$$
F(t)=A\left(\mathrm{e}^{-t / \tau_{1}}+\alpha \mathrm{e}^{-t / \tau_{2}}\right)
$$



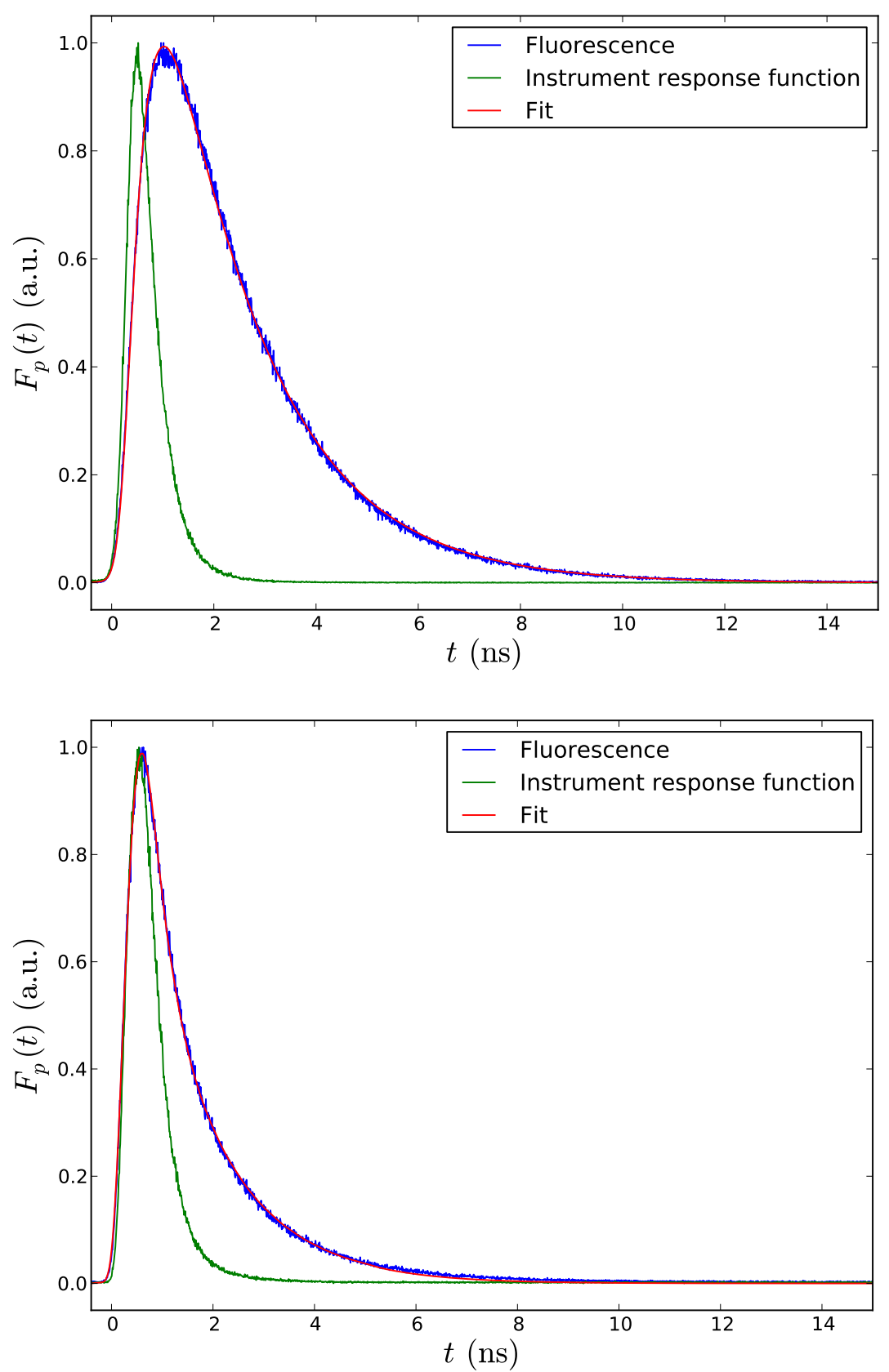

Figure 7.8.: Fluorescence lifetime measurements (blue) of Atto655 diffusing in a PBS solution through a focused laser beam in a confocal setup (up) and at the aperture probe with addition of Tween20 (down). The instrument response function (IRF, green) was measured the same way as the blue curve but without emission filter in order to mainly measure the excitation light. This lightwas also attenuated with a neutral glass filter. Fitting the functions was done by convolving the IRF with one and two exponential functions for the confocal and aperture probe case, respectively. 
where $\alpha$ reflects how much the fluorescence lifetime differs from a single exponential decay. If $\alpha<1$, the single exponential decay with fluorescence lifetime $\tau_{1}$ prevails. For the case of the confocal measurement where the lifetime of the fluorescent molecules is expected to be undisturbed, a purely single exponential fit was used and consequently $\alpha=0$. In such conditions, the fit value obtained for the lifetime of Atto655 is: $\tau_{1}=1.904 \pm 0.002 \mathrm{~ns}$, in good agreement with the value given by the manufacturer: $\tau_{1}=1.8 \mathrm{~ns}$ (Atto655 data sheet, measurement in water, [60]). However, if the fluorescence is measured from the Atto655 molecules diffusing at the aperture probe, the measured fluorescence signal changes significantly. It can no longer properly be fitted with the convolution of the IRF with a single exponential based lifetime. Instead, an additional exponential decay with a different lifetime has to be taken into account. Following equation 7.2, the values obtained by the fit in figure 7.8 (lower graph) are : $\tau_{1}=1.388 \pm 0.005, \tau_{2}=0.124 \pm 0.002$ and $\alpha=6.52 \pm 0.02$. These values not only show that the single exponential decay with the undisturbed fluorescence lifetime as measured in a confocal setup is heavily impaired, but also that it is largely reduced. This finding corroborates the idea of fluorescence enhancement at the aperture probe: spending less time in average in the singlet excited state, the decay rate $k_{21}$ increases and consequently the mean number of photon emitted by the fluorescent molecules also increases (equation 3.15).

As an additional consequence of the smaller fluorescence lifetime or equivalently increased decay rate $k_{21}$, the saturation intensity $I_{S}$ of the fluorescent molecule should increase (see equation 3.12). This could also be shown by comparing the count rate per molecules as a function of the excitation intensity in the case of a confocal excitation and in the case of an excitation through the aperture probe. The FCS measurements at four different intensities are given in figure 7.9. For each of the measurements, the background was estimated so that the $G(0)$ values can be corrected and the count rate per molecules calculated. For the confocal excitation, the noise is low and the corresponding FCS measurements were therefore not corrected for this small background. The count rate per molecules extracted from these measurements are shown in figure 7.10. The light power used to excite the fluorescent molecules is given by the power in the back focal plane of the objective focusing light in a PBS solution with the fluorescent molecule Atto655 with $10 \mathrm{nM}$ concentration and the power in the back focal plane of the objective focusing light into the aperture probe (see figure 5.7) for the NSOM-FCS measurements, where the aperture probe is immersed in a PBST solution with a Atto655 concentration of $250 \mathrm{nM}$.

This intensity dependent analysis of the count rate per molecules in the confocal case and in the NSOM case shows first the fluorescence intensity enhancement already mentioned before, due to the interaction of fluorescence light with the aperture probe that plays the role of a nanoantenna. It can clearly be seen from the inset of figure 7.10 that this count rate reaches a limit at $2 \mathrm{~mW}$ power because the slope of the curve approaches zero. In the case of the NSOM-FCS experiment, though the slope progressively decreases with higher excitation power, a further 


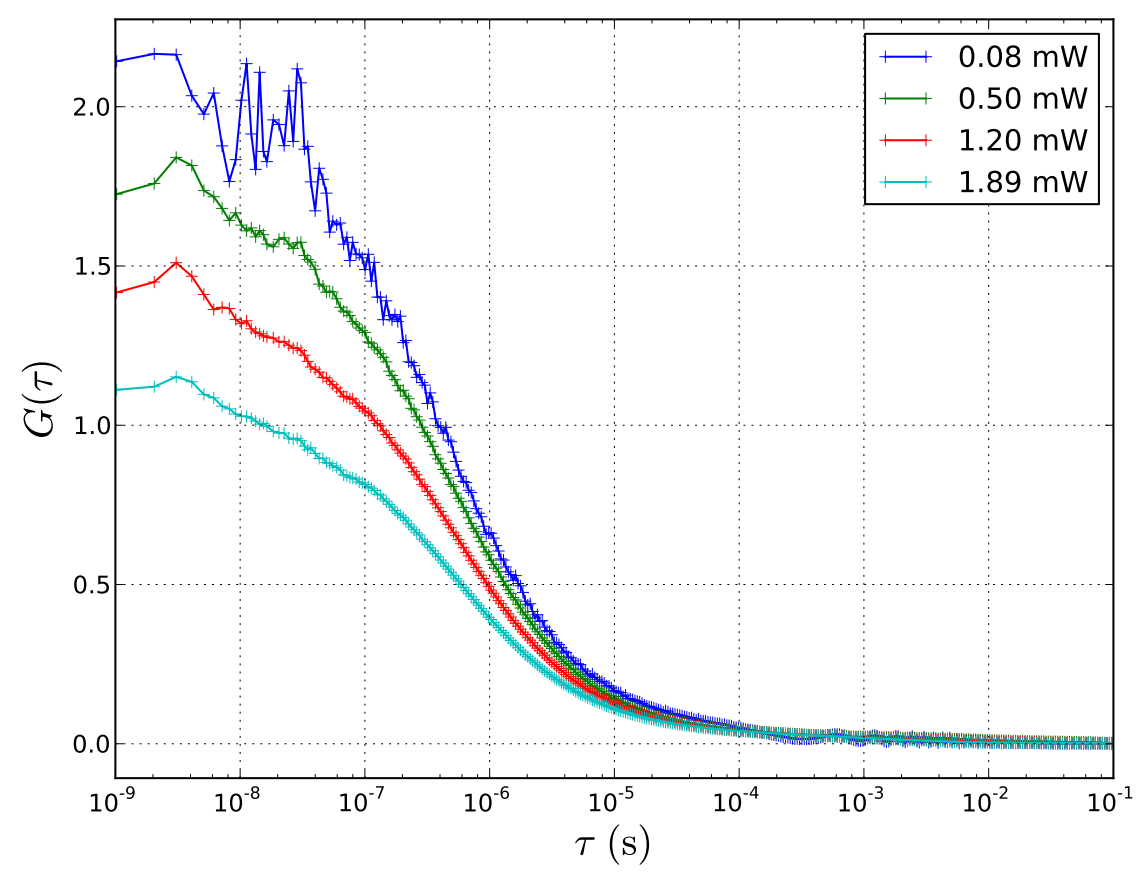

Figure 7.9.: Intensity dependent FCS measurements at a probe of aperture size $\sim 95 \mathrm{~nm}$ (as taken from an electron micrograph). The light power corresponds to the value measured at the back focal plane of the objective that focusses light in the aperture probe (see figure 5.7). The solution contains Atto655 in concentration $250 \mathrm{nM}$ in a PBST solution at $\mathrm{pH} \sim 7.4$. The amplitude of the measurements decreases as a result of the progressively saturating fluorescence emission of the Atto655 molecules that cannot compensate for the linear increase of the background. 


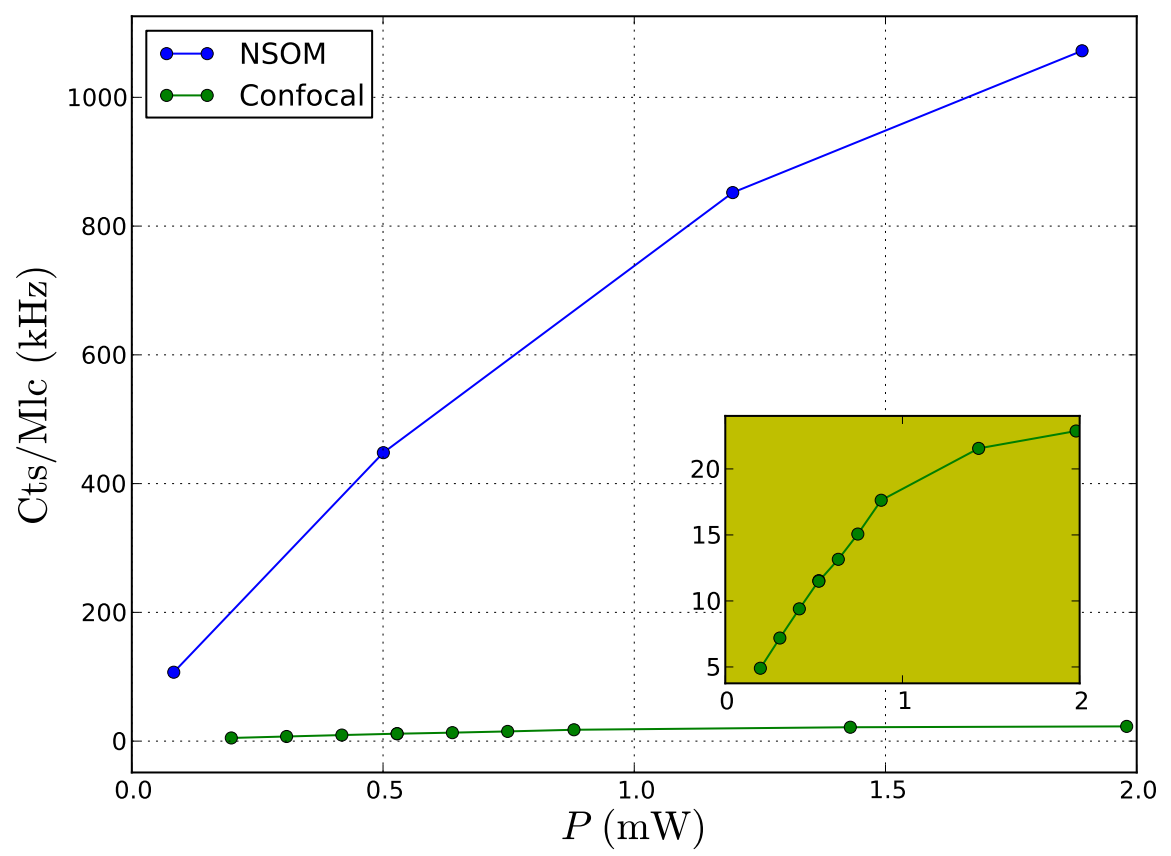

Figure 7.10.: Count rate per molecules (Cts/Mlc $(\mathrm{kHz})$ ) as a function of the excitation power at the back focal plane of the objective focusing light into the aperture probe (NSOM, blue curve) and of the objective focusing a collimated laser beam in the fluorescent molecules solution (confocal, green curve). The inset shows a zoom on the green curve. 
increase in count rate per molecules is still expected, possibly leading to an over two orders of magnitude higher count rate per molecules than in the confocal case. Similar experiments with nanohole milled in a gold film were carried out and also showed this fluorescence enhancement compared to a confocal excitation [18].

\subsection{Large intensity confinement at a TA probe}

Owing to its highly reduced excitation volume in comparison to the excitation volume from a focused laser beam, FCS measurements with an aperture probe opens the possibility of measuring dynamic processes of single molecules at high concentrations. In particular, biological processes such as enzymatic catalysis often have a dissociation constant in the micromolar range, which requires the different components of the association/dissociation reaction to be measured with micromolar concentrations in order to properly differentiate the different states of the components of the reaction [101].

In order to show the ability of measuring fluctuations of single molecules at high concentrations with a near-field aperture probe, such a probe was immersed in different PBS solutions of growing concentrations of Atto655 molecules: $250 \mathrm{nM}$, $2.5 \mu \mathrm{M}$ and finally $25 \mu \mathrm{M}$. The resulting cross-correlation of the fluorescence signal is shown in figure 7.11. In all three cases the power in the back focal plane of the objective placed before the probe in the excitation path (see figure 5.7) was held at relatively low levels: $95 \mu \mathrm{W}$ for the two lower concentrations and $40 \mu \mathrm{W}$ for the highest concentration.

These FCS measurements are characterized by the same course of the crosscorrelation function in the three cases that can be seen from the inset of figure 7.11 and which shows that the intensity distribution at the probe did not change between the three measurements. It is, however, astonishing that the amplitude of the cross-correlation function does not scale with the concentration as expected to from equation 3.55 since this amplitude is independent of the specific intensity distribution. A change in the amount of noise between the measurements at $250 \mathrm{nM}$ and $2.5 \mu \mathrm{M}$ Atto655 concentration is unlikely since the same intensity was used between the two measurements. Also the fact that the intensity distribution did not change, as can be seen from the conserved course of the two correlation functions, likely excludes structural changes at the aperture that would lead to another SNR (see precedent section).

In order to test the combined influence of fluorescence enhancement and fluorescence quenching at the TA probe and its metal interface near the aperture, respectively, fluorescence lifetime measurements were carried out for the three different concentrations. The results are given in table 7.2 obtained by fitting the lifetime measurements to equation 7.2. Average numbers of molecules in the excitation volume and mean count rate per molecules are also given for the three measurements from figure 7.11 assuming a negligible background justified by the very small excitation power used for these measurements. The parameters $\tau_{1}, \alpha$ and 


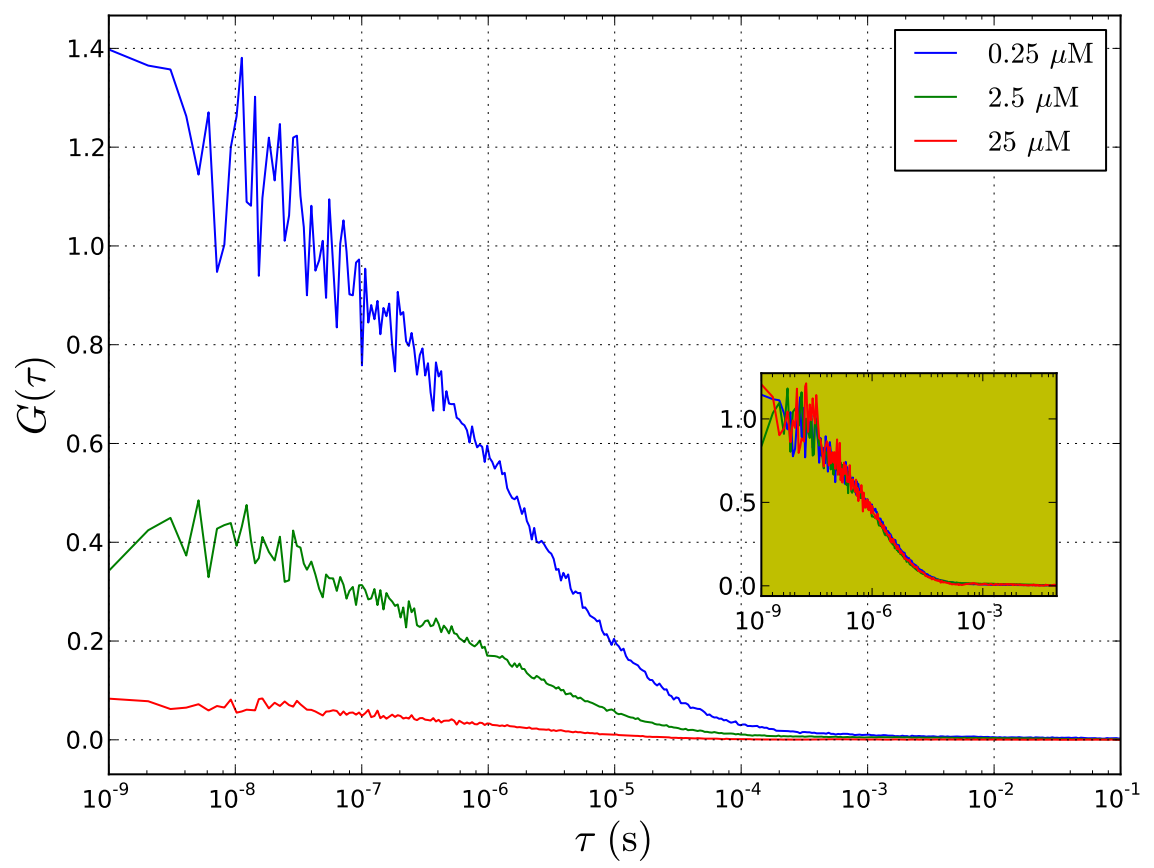

Figure 7.11.: NSOM-FCS measurements with a probe of aperture size $\sim 75 \mathrm{~nm}$ (as taken from an electron micrograph) of the diffusion of Atto655 in a PBS solution ( $\mathrm{pH} \sim 7.4$ ) and at different concentrations: $250 \mathrm{nM}$, $2.5 \mu \mathrm{M}$ and $25 \mu \mathrm{M}$. The inset shows the same measurements but normalized. The power at the back focal plane of the focusing objective is $95 \mu \mathrm{W}$ for the measurements at $250 \mathrm{nM}$ and $2.5 \mu \mathrm{M}$ and $40 \mu \mathrm{W}$ at $25 \mu \mathrm{M}$. 


\begin{tabular}{|c|c|c|c|}
\hline Concentration & $0.25 \mu \mathrm{M}$ & $2.5 \mu \mathrm{M}$ & $25 \mu \mathrm{M}$ \\
\hline$\tau_{1}(\mathrm{~ns})$ & $1.418 \pm 0.007$ & $1.433 \pm 0.005$ & $1.441 \pm 0.005$ \\
$\alpha$ & $14.4 \pm 0.2$ & $7.7 \pm 0.1$ & $18.8 \pm 0.3$ \\
$\tau_{2}(\mathrm{~ns})$ & $0.101 \pm 0.002$ & $0.114 \pm 0.002$ & $0.078 \pm 0.001$ \\
$\langle N(t)\rangle$ & $\sim 0.82$ & $\sim 2.4$ & $\sim 14.5$ \\
$\mathrm{CRM}(\mathrm{kHz})$ & $\sim 79$ & $\sim 10.1$ & $\sim 42$ \\
\hline
\end{tabular}

Table 7.2.: Results of lifetime measurements at $40 \mathrm{MHz}$ excitation frequency for the three different concentrations of figure 7.11: $\tau_{1}, \alpha$ and $\tau_{2}$ were obtained by fitting the measurements to equation 7.2. Also the average number of molecules in the excitation volume $(\langle N(t)\rangle)$ and the count rate per molecules (CRM) extracted from the FCS measurements of figure 7.11 are given assuming a negligible noise. Note that for the measurement at $25 \mu \mathrm{M}$, the power used to excite the molecules is a factor $\sim 2.4$ smaller than the power used for the measurement at $2.5 \mu \mathrm{M}$, which corresponds to the ratio of the count rate per molecules for these two measurements.

$\tau_{2}$ do not remain constant, contrary to the expectation. At high concentrations, the highly reduced fluorescence lifetime cannot be attributed to a self-quenching phenomenon since the mean distance between Atto655 molecules $C_{0}^{-1 / 3} \sim 40 \mathrm{~nm}$ $\left(C_{0}\right.$ is the molecule concentration) is still high compared to the few nanometers required for an energy transfer between two molecules [102].

Additionally, to exclude the possibility of errors in the preparation of the Atto655 molecules, the UV-Vis absorption spectrum of the solutions with nominal concentrations of $2.5 \mu \mathrm{M}$ and $25 \mu \mathrm{M}$ were recorded directly after the FCS and lifetime measurements to determine their concentration independently. The absorption spectra are shown in figure 7.12. The maximum of the optical density (OD, defined as $\mathrm{OD}=-\log \left(\frac{I_{f}}{I_{0}}\right)=\epsilon_{\text {ext }} l c$, see section 3.1.2) in the two measurements was used to derive the concentration of the two solutions. The value found with the extinction coefficient of Atto655 at its maximum absorption $\left(\epsilon_{\text {ext }}=125000 \mathrm{M}^{-1} \mathrm{~cm}^{-1}\right)$ and a cuve length of $l=0.3 \mathrm{~cm}$ are $3.28 \mu \mathrm{M}$ and $29.4 \mu \mathrm{M}$. The factor of $\sim 9$ between these two values is still $50 \%$ higher than the ratio of mean number of molecules in the excitation volume for the FCS measurements with these two different concentrations.

Even if the evolution of the amplitude of the cross-correlation functions with the concentration of the fluorescent molecules is still poorly understood, these measurements show promising perspectives towards the measurement of single molecule dynamics at concentrations up to $100 \mu \mathrm{M}$. This property stems from the highly confined fields at the near-field aperture probe, which simultaneously means that these dynamic measurements are made spatially highly resolved. 


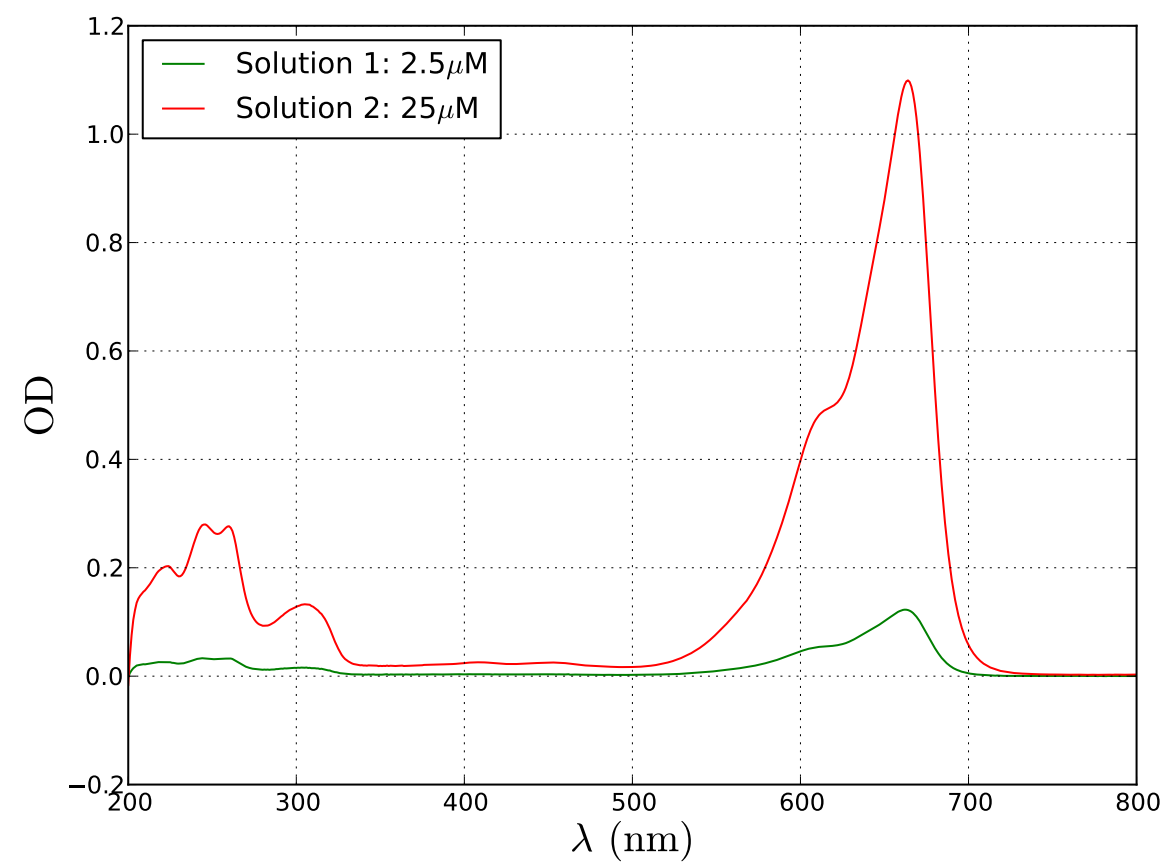

Figure 7.12.: Absorption spectrum of the solutions used to measure the FCS resp. lifetime measurements of figure 7.11 resp. of table 7.2 for the nominal concentrations of $2.5 \mu \mathrm{M}$ resp $25 \mu \mathrm{M}$. The evaluation of the optical density at the maximum of absorption gave $3.28 \mu \mathrm{M}$ resp. $29.4 \mu \mathrm{M}$. 


\subsection{High time resolution of NSOM-FCS}

In section 4.2.3, it was shown that in the simulation of the intensity at the nearfield aperture probe, mainly the exponential decaying fields in the axial direction shape the course of the correlation function. In this section, the experimental FCS measurements are presented. In order to be able to compare the varying experimental results between each other independently of any fit function, a criterion was adopted to give a measure of how confined the intensity at the probe aperture is. This criterion is based on the ratio of the amplitude of the cross-correlation function at a lag time of $0.1 \mu \mathrm{s}$ to the amplitude at lag time $10 \mu \mathrm{s}$. The higher the value, the more confined should be the intensity. The best value was reached for the measurement shown in figure 7.13.

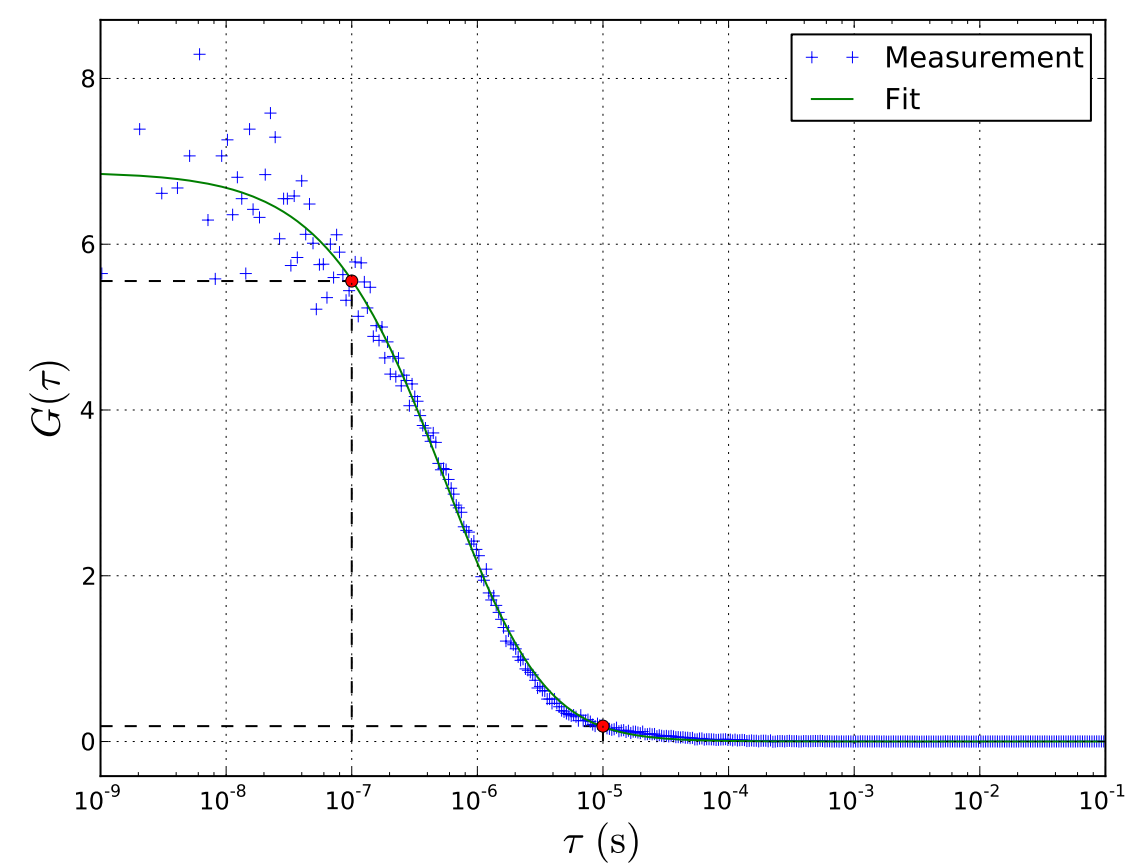

Figure 7.13.: FCS measurement of Alexa647 (100 nM) in a PBS solution at an aperture probe of approximately $45 \mathrm{~nm}$ aperture size (as taken from an electron micrograph). The power at the back focal plane of the focusing objective is $\sim 2.5 \mathrm{~mW}$ and a total count rate of $\sim 16 \mathrm{kHz}$ was measured during $120 \mathrm{~s}$. Fitting the experimental curve was done with equation 4.22. The two red points represent the two values of the amplitude of the cross-correlation function, the ratio of which was used to evaluate the field confinement at the aperture.

Fitting the cross-correlation curve with equation 4.22 results in a decay length in axial direction of $12 \pm 1 \mathrm{~nm}$ and a lateral extension of $43 \pm 2 \mathrm{~nm}$. This lateral extension was shown in section 4.2.3 to represent the radius of the equivalent Gaussian excitation profile by defining this radius as the point of the excitation profile, 
where the intensity decayed to $1 / \mathrm{e}^{2}$. This value is extremely small considering that it represents an average extension at a few nanometers of the aperture and therefore should be at least the size of the aperture plus a few ten nanometers due to leakage of excitation light through the side walls of the gold coating (see section 4.2.3).

In the case of the fluorescent molecules Atto655, that are lighter than Alexa647 (the molecular mass of Atto655 is $634 \mathrm{~g} / \mathrm{mol}$ [60] against $1250 \mathrm{~g} / \mathrm{mol}$ for Alexa647 [103]), the best confinement is obtained for the measurement shown in figure 7.14. This measurement features a very different shape of the intensity distribution. The fit results are $4.80 \pm 0.07 \mathrm{~nm}$ for the decay length $d$ in axial direction and $115 \pm 5 \mathrm{~nm}$ for the lateral extension of the radius at $1 / \mathrm{e}^{2}$ decay of the equivalent Gaussian profile, whereas the fit cannot reproduce the correlation at longer lag times, that may be due to temporary binding of the fluorescent molecules at the glass surface (see section 7.5).

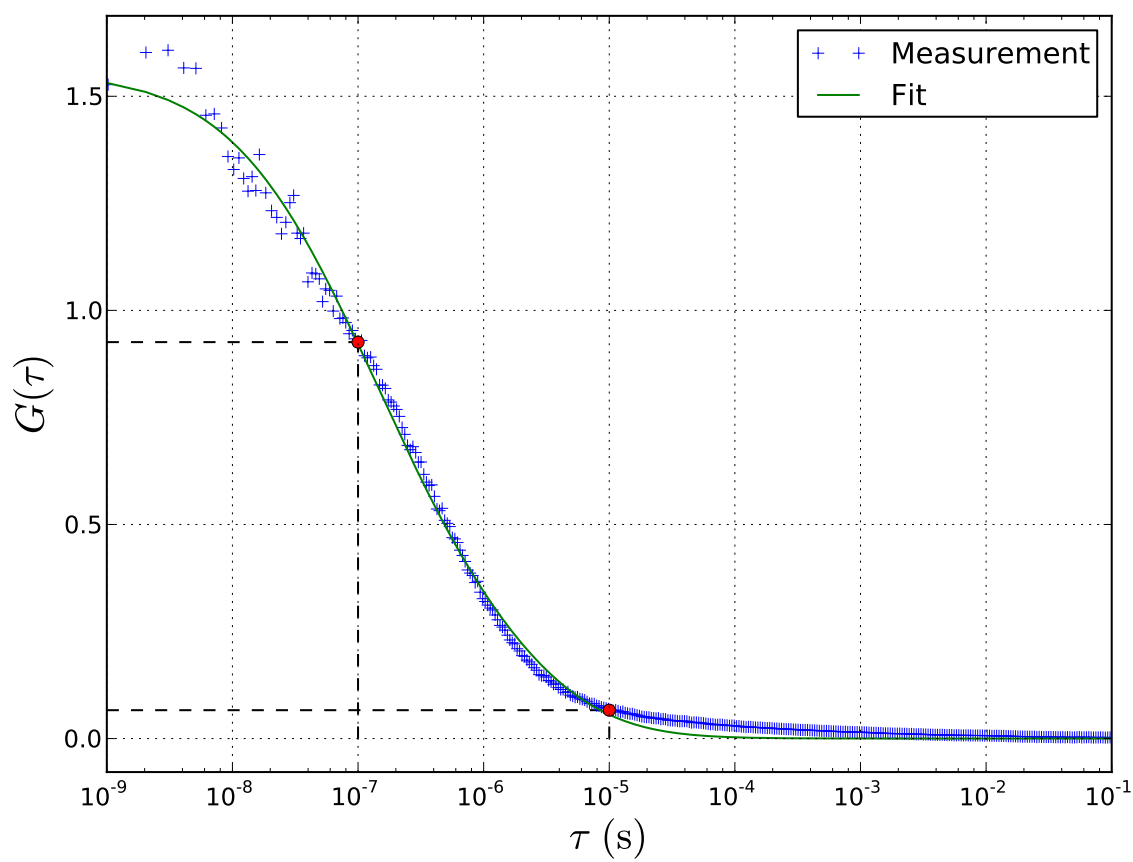

Figure 7.14.: FCS measurement of Atto655 (100 nM) in a PBS solution at an aperture probe of approximately $60 \mathrm{~nm}$ aperture size (as taken from an electron micrograph). The power at the back focal plane of the focusing objective is $\sim 1.9 \mathrm{~mW}$ and a total count rate of $\sim 250 \mathrm{kHz}$ was measured during $60 \mathrm{~s}$. The fitting of the experimental curve was done with equation 4.22 . The two red points represent the two values of the amplitude of the cross-correlation function, the ratio of which was used to evaluate the field confinement at the aperture.

Between these two extreme values of the decay length of the intensity in the axial direction, some measurements showed the same course as in the simulations at 
the model aperture probe (see section 4.2.3). For example the FCS measurement presented in figure 7.15 can be very well fitted by simulating the fluorescence signal stemming from molecules diffusing in solution and excited through the intensity distribution simulation explained in section 4.2.2. The simulated correlation function was already fitted to equation 4.22 in section 4.2 .3 and the fit parameters were found to be $7.22 \pm 0.05 \mathrm{~nm}$ for the decay length in axial direction and $113 \pm 2 \mathrm{~nm}$ for the radius at $1 / \mathrm{e}^{2}$ decay of the equivalent Gaussian profile in the lateral plane.

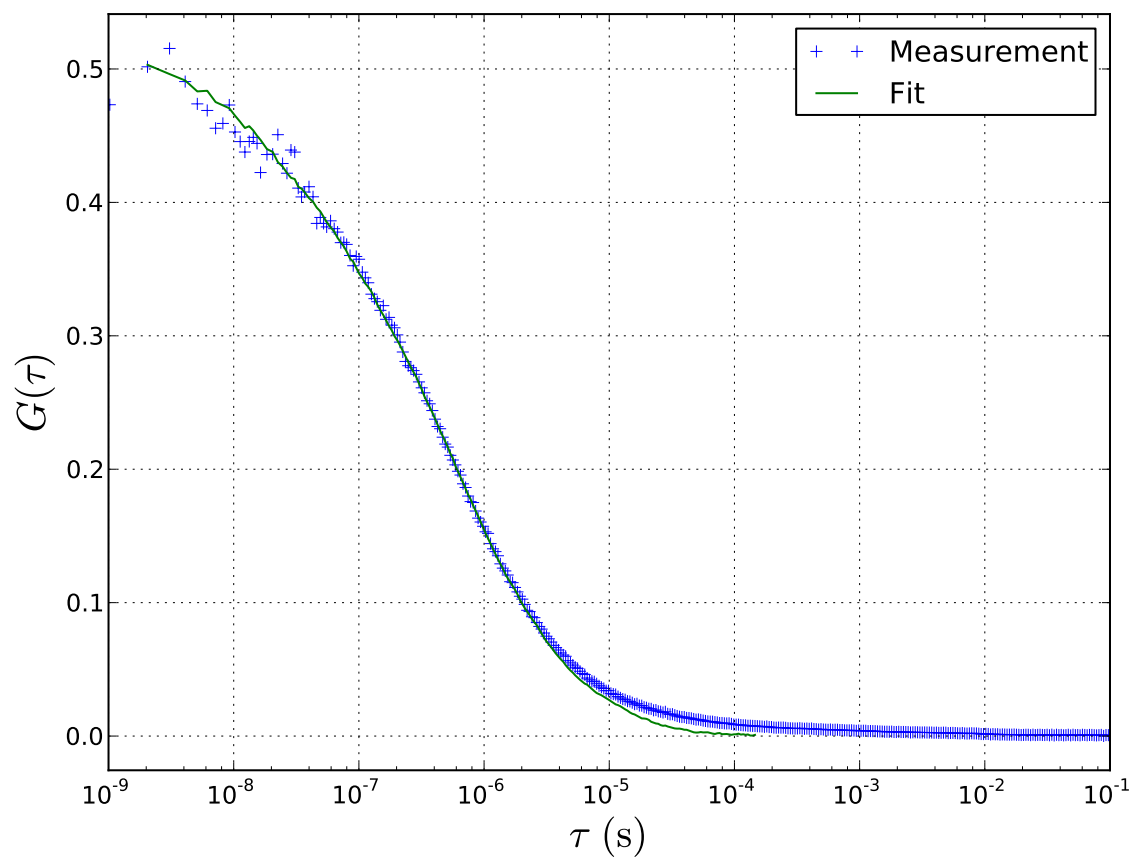

Figure 7.15.: FCS measurement of Atto655 $(2,5 \mu \mathrm{M})$ in a PBST solution at an aperture probe of approximately $95 \mathrm{~nm}$ aperture size (as taken from an electron micrograph). The power at the back focal plane of the focussing objective is $\sim 95 \mu \mathrm{W}$ and a total count rate of $\sim 680 \mathrm{kHz}$ was measured during $60 \mathrm{~s}$. Fitting the experimental curve was done with a simulated correlation function using the simulated intensity distribution at the triangular aperture from section 4.2.2.

These results show that not only the amplitude of the cross-correlation function has a complicated dependence on the size of the aperture probe (section 7.2) but also the course of the function and therefore the parameters describing the confinement of the field at the aperture probe. Deviations of the fits and the FCS measurements at longer lag times, on the scale of which the fluctuations due to diffusional processes in the confined excitation field are not correlated anymore, can also sometimes be observed as for example in figure 7.14 and 7.15 for lag times of about $0,1 \mathrm{~ms}$. The possible reason for this is exposed in the next section. 


\subsection{Change of the molecule's dynamic at the TA probe}

In order to characterize the intensity distribution at the aperture probe it is best to measure a fluorescence signal fluctuating only because of the diffusion process of the fluorescent molecules under the aperture. Fluctuating but not correlated signal such as background noise can be relatively easily accounted for by multiplying the amplitude of the correlation function by a constant factor (see section 7.2). Without this correction, the intensity distribution can still be retrieved but the assessment of the mean number of molecules in the excitation volume and therefore interesting data like mean count rate per molecules is impaired if the background noise is not measured independently. On the contrary, fluctuating correlated signal other than resulting from diffusion process can severely alter the course of the correlation function and prevent to determine correctly the intensity distribution.

\subsubsection{Effect of the solvent}

To exemplify this point, figure 7.16 shows two simulations of an FCS measurement at the model aperture probe described in section 4.2.2.

The two simulations were conducted with exactly the same parameters (see figure 7.16) except for the binding mechanism of the fluorescent molecules at the glass interface of the probe. In one case, it is neglected, in the other case, a binding probability of $1 \%$ with a mean binding time of $0.1 \mathrm{~ms}$ is set. The difference in the course of the correlation curve is tremendous and has nothing to do anymore with the steep decay of the fluorescence correlation due to diffusion of the fluorescent molecules in the high intensity gradient around the aperture.

This kind of binding phenomenon could be observed in two different situations. Firstly, by the comparison of FCS measurements using the fluorescent molecules Alexa 647 in an ethanolic solution and a PBS solution. Secondly, and to a less extent, with the fluorescent molecules Atto 655 in buffer solutions of varying $\mathrm{pH}$. Two exemplary measurements for the molecules Alexa 647 are shown in figure 7.17. The measurement in the PBS solution shows the usual steep decay of the cross-correlation curve resulting from the diffusion processes in the high confined excitation volume around the aperture. Much different is the measurement in the ethanolic solution, for which additional correlations at large lag times appear. The correlation function is very similar to the simulated correlation function of figure 7.16, which suggests also a binding mechanism of the fluorescent molecules at the glass interface.

Also the comparison of the two different time traces is very instructive and is given in figure 7.18 for a time bin of $2.15 \mathrm{~ms}$. While the fluorescence signal stemming from Alexa 647 in the PBS solution is characterized by small fluctuations on the microsecond scale that averaged out on a time scale of a few milliseconds and give a relatively constant fluorescent signal, the fluorescence emitted in the ethanol 


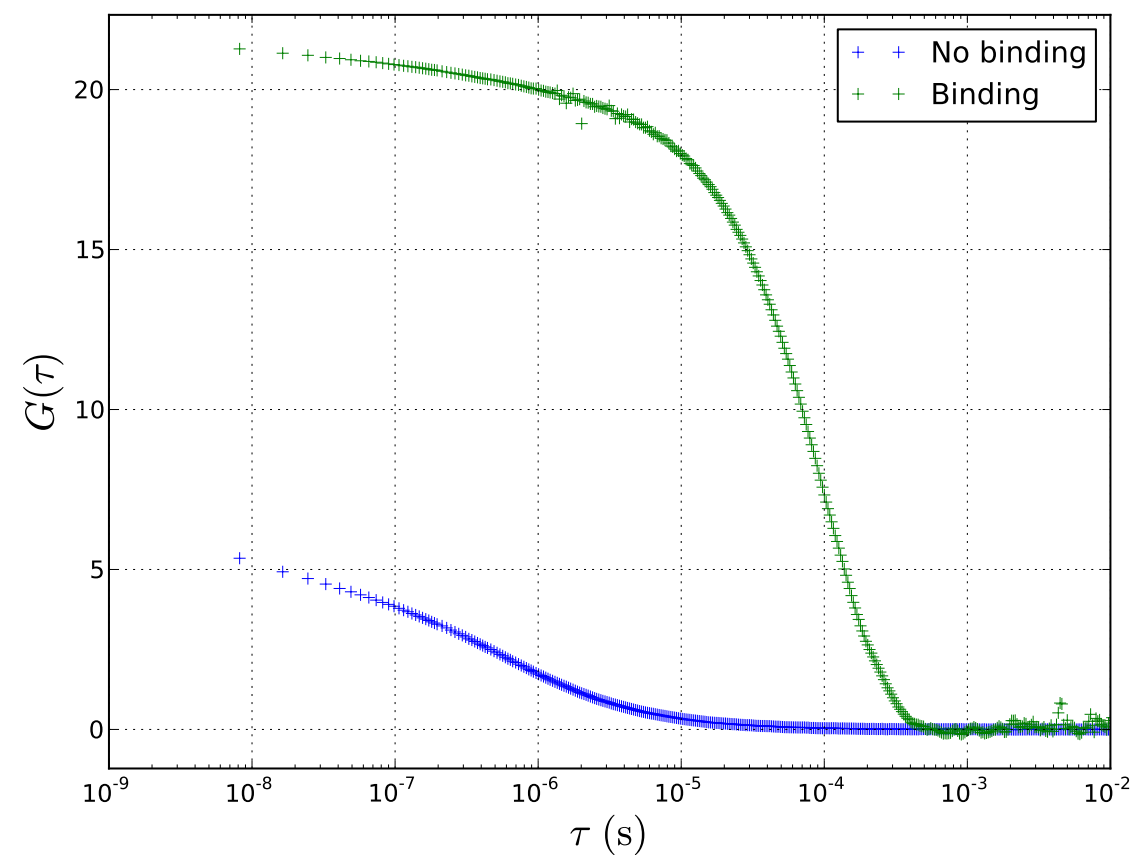

Figure 7.16.: Simulation of an FCS measurement at the model aperture probe described in section 4.2.2. The simulation volume extends $1,5 \mu \mathrm{m}$ away from the probe axis in the lateral plane and $1.5 \mu \mathrm{m}$ away from the aperture end in axial direction. The signal of 1,000 molecules diffusing in the volume is autocorrelated to obtain these curves. Both measurements are simulated with an excitation intensity of $1,000 \mathrm{~kW} / \mathrm{cm}^{2}$ at maximum intensity and at a wavelength of $640 \mathrm{~nm}$, a negligible background intensity of $1 \mathrm{kHz}$ and the photophysical parameters of Atto 655 at this excitation wavelength, i.e. $\sigma_{\mathrm{abs}}=315 \cdot 10^{-18} \mathrm{~cm}^{2}$, $k_{21}=1.85 \cdot 10^{9} \mathrm{~s}^{-1}, k_{21}=1 \mathrm{~s}^{-1}$ (reflecting a negligible triplet occupation) and $k_{32}=0.5 \cdot 10^{6} \mathrm{~s}^{-1}$ (although irrelevant as triplet excitation is very unlikely to happen). The two simulations were carried out $1.5 \mathrm{~s}$. The only difference between the two simulations is the simulation of an additional binding process at the glass interface with a probability of $1 \%$ and a mean binding time of $0.1 \mathrm{~ms}$ in one case and no binding in the other case. The count rate is 1.75 times higher for the simulation with binding events. 


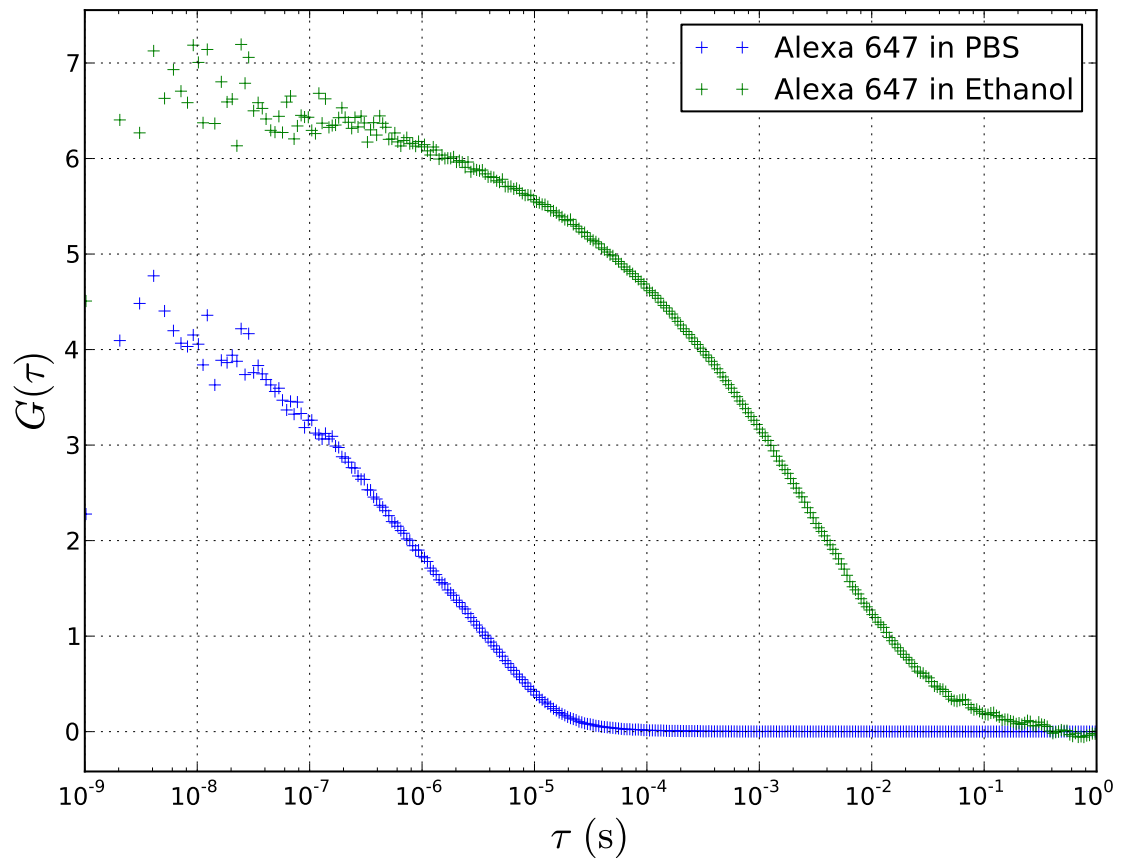

Figure 7.17.: FCS measurements of Alexa 647 at $100 \mathrm{nM}$ concentration in two different solutions: PBS (blue) and ethanol (green). The measurements were taken with two different gold coated aperture probes featuring the same aperture size of $90 \mathrm{~nm}$ as measured from their respective electron micrographs. 
solution not only show additional fluctuations on a much longer time scale of some hundreds of milliseconds. These fluctuations are also characterized by a high intensity of the fluorescence signal. This suggests that the fluorescent molecules transiently bind to the glass aperture where they are excited with the highest intensity. It has to be noted that FCS measurements in ethanol show also a greater variability in the amplitude of the correlation curve for different measurements in otherwise same conditions. But this is due to the long correlation time of the additional fluctuations (some hundreds of milliseconds), which impairs a good statistical accuracy for the acquisition time of $60 \mathrm{~s}$ of this FCS measurement [98].
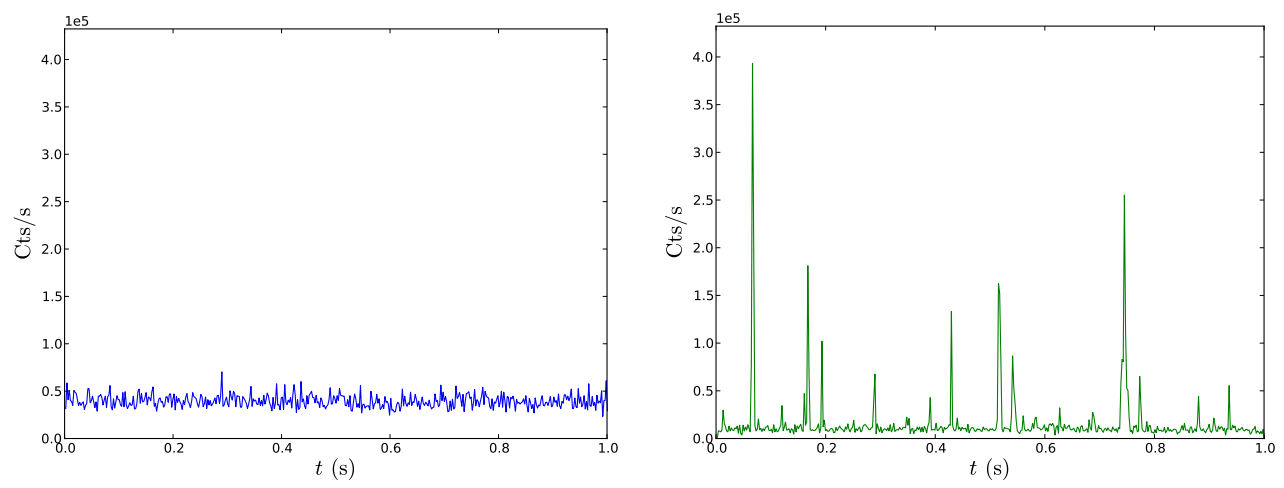

Figure 7.18.: Time trace of the two FCS measurements shown in figure 7.17 during one second with a time bin of $2.15 \mathrm{~ms}$. Left: in the PBS solution. Right: In ethanol. In ethanol, many events during some hundreds of milliseconds occur for which the count rates reach very high values increasing from about $23000 \mathrm{Cts} / \mathrm{s}$ to some $100000 \mathrm{Cts} / \mathrm{s}$, i.e. a transient increase of about one order of magnitude.

Measurement of the fluorescence lifetime of Alexa 647 in the ethanol solution at the aperture probe shows a strong increase of the fluorescence decay rate compared to its value measured in a confocal setup (see figure 7.19). In ethanol the fluorescence lifetime is known to increase compared to its value in water from 1.04 ns to $1.51 \mathrm{~ns}$ [70]. The measured value of $1.469 \pm 0.004 \mathrm{~ns}$ is in accordance with the reported value. Strikingly, when measured while diffusing at the aperture probe, the lifetime is heavily affected to an even greater extent as for the fluorescent molecule Atto 655 in aqueous solution as reported in figure 7.8 and table 7.2. The fit parameters are $\tau_{1}=1.57 \pm 0.03 \mathrm{~ns}, \tau_{2}=0.057 \pm 0.001 \mathrm{~ns}$ and $\alpha=167 \pm 3$ (see equation 7.2). This result combined with the high increase of the fluorescence count rate for binding event shown in figure 7.18 back up the hypothesis of fluorescence enhancement at the aperture probe. The different amount of change of the fluorescence lifetime for Atto 655 in aqueous solution (figure 7.8) and Alexa 647 in ethanol (figure 7.19) suggests that this fluorescence enhancement is also highly dependent on the nature of the fluorescent molecule or/and the solution. 

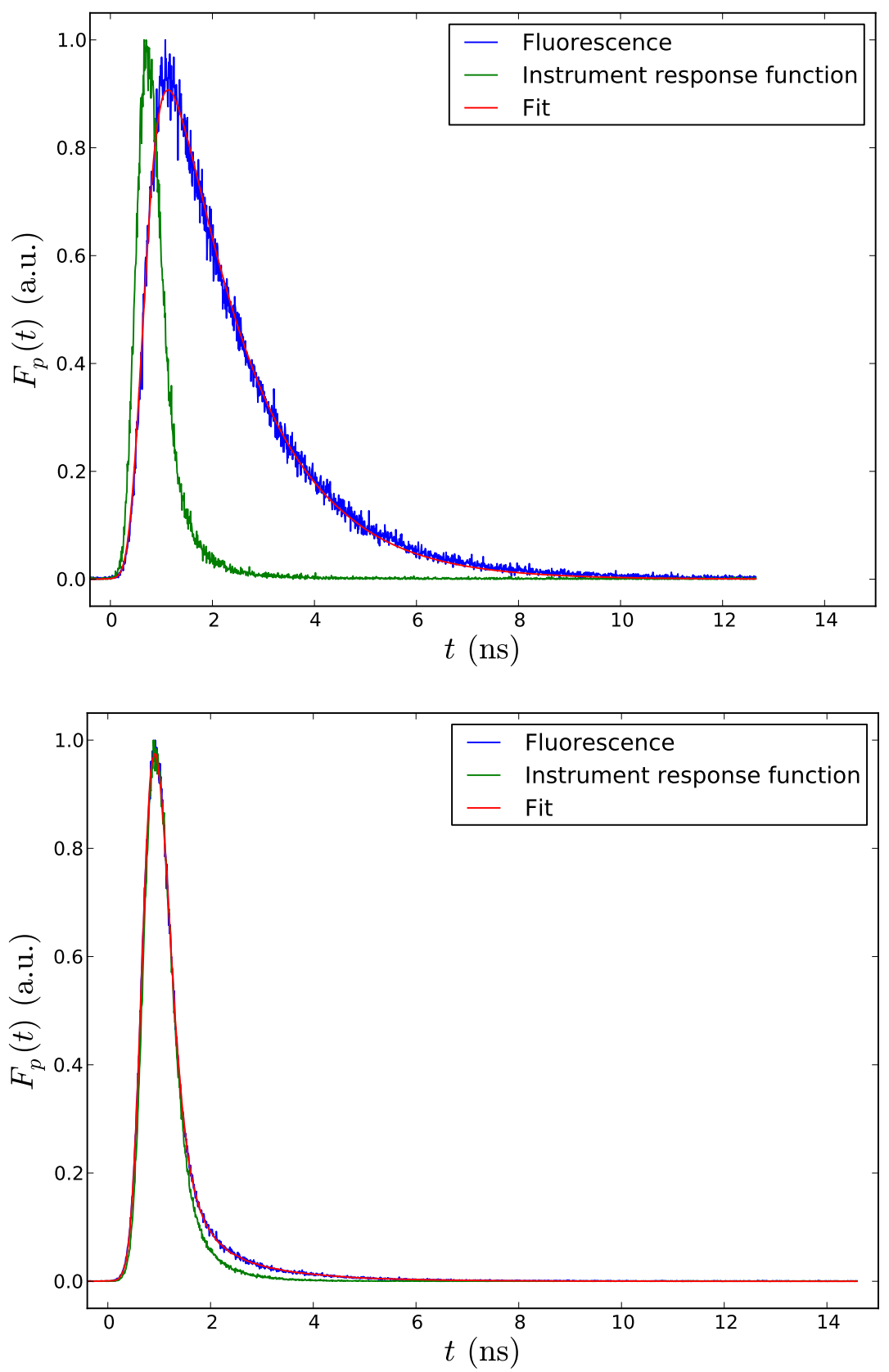

Figure 7.19.: Fluorescence lifetime measurements (blue) of Alexa 647 diffusing in a focused laser beam in a confocal setup (up) and at the aperture probe (down) both in an ethanol solution. The instrument response function (IRF, green) was measured the same way as the blue curve but without emission filter in order to measure mainly the excitation light and with a neutral glass filter to attenuate it. Fitting the functions was done by convolving the IRF with a single and two exponential functions for the confocal and the aperture probe case, respectively. 


\subsubsection{Effect of the $\mathrm{pH}$}

In the case of Atto655 in an aqueous solution, also several FCS measurements showed a correlation at longer times even though the appearance of the events is less strong than in the case of Alexa 647 in an ethanolic solution. Analyzing the time trace of these FCS measurements shows also events of longer duration (10 to $10 \mathrm{~ms}$ ) where the fluorescence signal is greater than in average but this signal increase is much smaller as the signal increase that can be observed in figure 7.18. Also the frequency of an event is not so high. These two observations suggest that also in this case the fluorescent molecules transiently bind to the glass surface of the aperture probe but with a much smaller probability as Alexa 647 in ethanol. Different measurements were carried out in buffered solutions to determine which parameters among $\mathrm{pH}$, buffer strength and buffer type have an effect on these binding events and are illustrated in figure 7.20, 7.21 and 7.22. Although these are single measurements with an acquisition time of $60 \mathrm{~s}$, that vary all the more that the binding event have a large mean binding time, these measurements are considered to be representative.

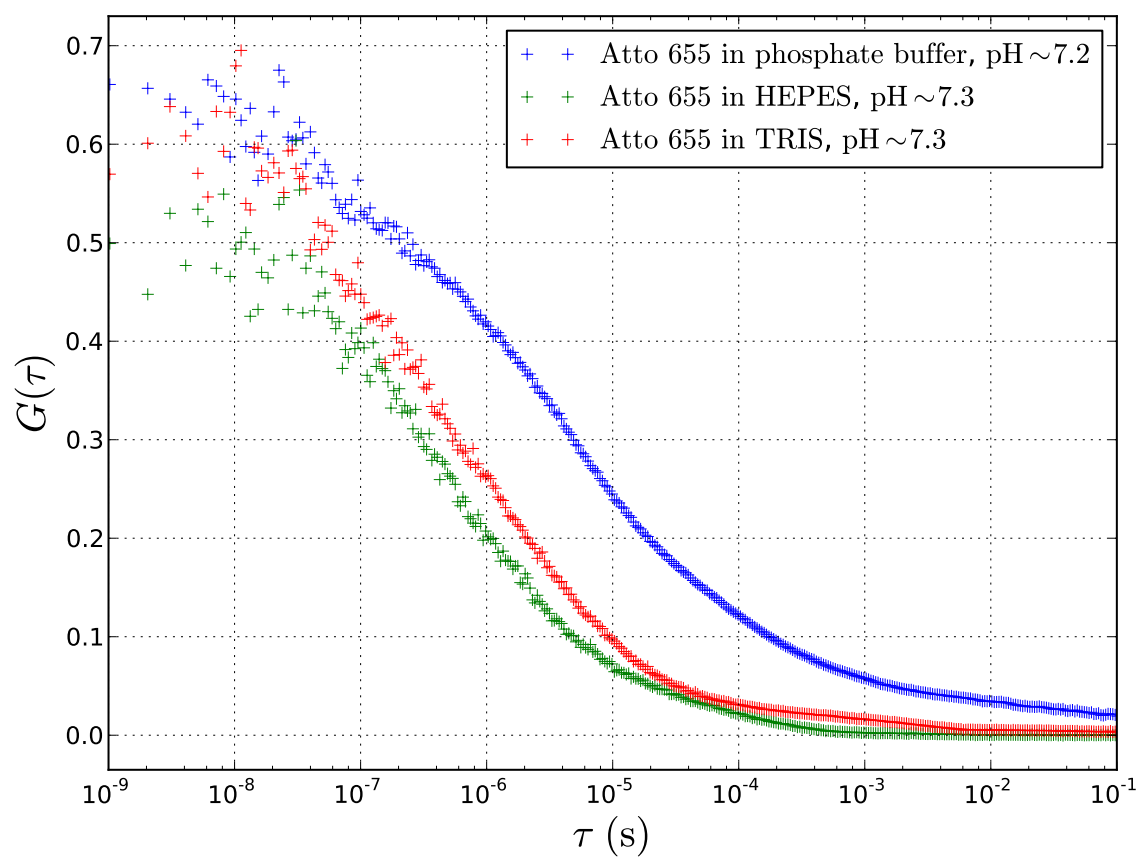

Figure 7.20.: FCS measurements with a gold coated probe of aperture size $\sim 75 \mathrm{~nm}$ (from an electron micrograph) and with a solution of Atto 655 $(2,5 \mu \mathrm{M})$ in an aqueous solution prepared with different buffer at $\mathrm{pH} \sim 7,2-7,3$. Every buffer solution was prepared with $100 \mathrm{mM}$ of the buffer substance.

In figure 7.20, the influence of the buffer type at fixed $\mathrm{pH}$ and buffer strength was investigated. The three measurements feature long correlation time to a different 


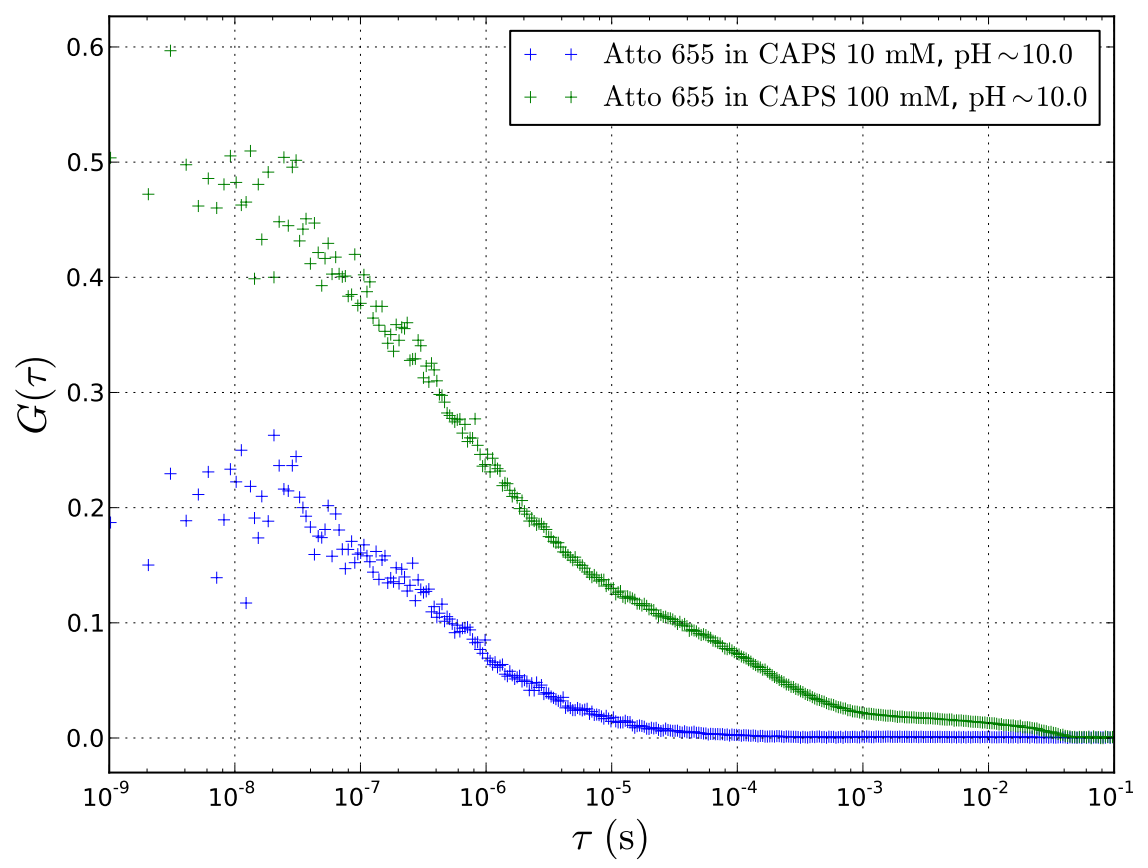

Figure 7.21.: FCS measurements with a gold coated probe of aperture size $\sim 75 \mathrm{~nm}$ (from an electron micrograph) and with a solution of Atto 655 $(2,5 \mu \mathrm{M})$ in an aqueous solution prepared with the buffer substance CAPS at $\mathrm{pH} \sim 10$ and for different buffer strengths. 


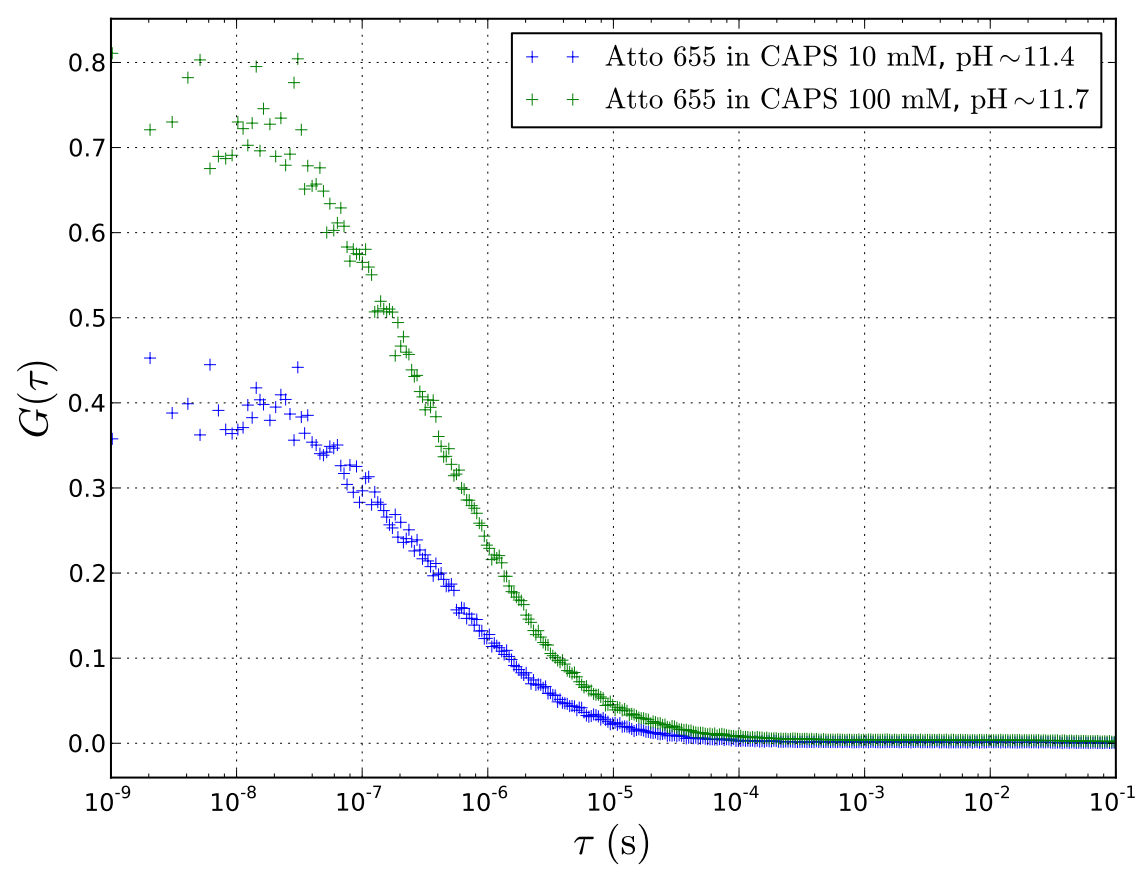

Figure 7.22.: FCS measurements with a gold coated probe of aperture size $\sim 75 \mathrm{~nm}$ (from an electron micrograph) and with a solution of Atto 655 $(2,5 \mu \mathrm{M})$ in an aqueous solution prepared with the buffer substance CAPS at high $\mathrm{pH}$ value and for different buffer strengths. 
extent but these different measurement conditions do not seem to totally hinder the binding events. Figure 7.21 and 7.22 show FCS measurements with a progressive increase of the $\mathrm{pH}$ value of the buffer solution. The higher the $\mathrm{pH}$, the steeper the correlation function decays and the more unprobable a binding event becomes. Buffer strength or buffer type do not seem to have a great influence on these binding events.

A possible explanation to the occurences of these binding events is a weak electrostatic interaction between the fluorescent molecules and the glass surface. The glass surface is originally treated in an alcaline solution (Hellmanex, see section 5.1.1) which very likely increases the density of $\mathrm{SiOH}^{(-)}$group at the glass surface and provides a great hydrophilicity for the glass surface after this treatment. However, the fluorescent molecules have no published structure so that it is hard to validate or invalidate the hypothesis of an electrostatic interaction. It is solely known that Atto 655 is a zwitterionic molecule [60] and Alexa 647 is supposed to be negatively charged due to a sulfonation process of some cyanine dyes (category of dye to which Alexa 647 is affiliated according to reference [70]). This latter supposition would actually weaken the idea of a binding process relying on an electrostatic interaction. Moreover, if the effect is only due to a high charge density on the glass surface following a chemical treatment, the effect should also be well observed in an FCS experiment conducted with a TIRF setup (see figure 4.7), which has not been reported to this extent (to our knowledge).

An alternative explanation to this transient binding and where the specific physical characteristic of the near-field aperture probe plays an important role, can be derived from similar findings: the adsorption of DNA double strands on gold nanoparticles [104]. These strands actually have an anionic character through the phosphate groups of the nucleotide side chain. The gold nanoparticles bear by way of their fabrication process also a negative charge on their surface. These two elements would invalidate the electrostatic interaction theory. Sandström et al. explain this adsorption by an ion-induced dipole interaction. The charges of th phosphate groups of DNA could induce a dipole in the highly polarizable gold particles. The intensity of this short ranged mechanism exceeds the electrostatic repulsion only for a few nanometers distance between DNA and gold nanoparticles. This polarization mechanism could possibly play a role in the transient binding of fluorescent molecules at the triangular aperture of the near-field aperture probe. 


\section{Conclusion}

Prelude to all NSOM-FCS measurements obtained for this thesis was a reliable and reproductible fabrication process for triangular aperture (TA) probes for measurements in aqueous buffer. The probe fabrication including an almost perfect aperture formation using a focused ion beam could be carried out with a yield of nearly $100 \%$. Light focusing into the probe resulted for few probes in ill defined diffraction patterns due to light leakage through the metal coating, however, an overall yield better than $50 \%$ was still achieved with the newly developed fabrication process.

As a result, the TA probes could be used to image single nuclear pores of a free standing nuclear membrane with sub-50-nm resolution. Imaging the membrane was the key to the accurate localization of single nuclear pores. This localization allowed us to place the TA probe over a single nuclear pore and carry out NSOMFCS measurements of the translocation process at such a structure for the first time. The highly confined and rapidly decaying intensity at the TA probe was an inevitable prerequisite for this study and led to the determination of minimum values for the effective diffusion range and the apparent diffusion coefficient of proteins in a pore. Such experimental data of the dynamic structure of a pore are indispensable for a better understanding of the translocation of proteins through the nuclear membrane.

Furthermore, major insight could be gained concerning the intensity distribution at a TA probe. Using available numerical calculations of the intensity distribution at a simplified model of a triangular aperture in a gold film, FCS simulations were carried out. The analysis of the autocorrelation function resulting from the simulation of the free diffusion of fluorescent molecules at this model aperture probe led us to a heuristic model for an accurate description of the FCS curve. On the one hand, this heuristic model is able to describe the rapid decay of the FCS curve at small lag times using the analytical FCS curve resulting from a diffusion in a one dimensional exponential decaying field as used, for example, in total internal reflection fluorescence (TIRF) measurements. On the other hand, the much faster decay as compared to TIRF-FCS on larger lag times is successfully described by the analytical FCS curve resulting from the diffusion in a two dimensional Gaussian intensity distribution. The heuristic model is shown to correspond very well with simulated FCS curves and therefore provide a simple equivalent description of the excitation volume at the TA probe. Using this equivalent description, a characteristic size of the excitation volume at the probe could be evaluated to 3 attoliters. Such an ultra-small excitation volume even makes it feasible to observe single molecule dynamics at phisiologically relevant protein concentrations in the 
micromolar range. The time resolution achieved by NSOM-FCS using triangular apertures was evaluated to reach $300 \mathrm{~ns}$.

During this thesis a large number of successful NSOM-FCS measurements have been collected thereby providing a solid basis for the comparison of experimental and theoretical results in this new field. In general, the theoretical simulations show a good agreement with the experimental results. However, the expected reduction of excitation volume with decreasing aperture size was not always observed. This different size scaling is shown to result from two different contributions. Firstly, a large background was measured which stems from luminescence of the gold coating of the TA probe. This luminescence very probably originates from near-field mediated intraband transitions in the metal, possibly enhanced by corrugations in the metal coating. Secondly, antenna effects may also contribute to the absence of size scaling and, to demonstrate this, lifetime measurements were carried out showing a large decrease of the fluorescence lifetime of the molecules diffusing at the probe. Simultaneously, measurements on the count rate per molecules showed increased values so that an enhanced fluorescence resulting from an interaction of the molecules with the TA probe seems obvious. Finally, it was shown that, depending on the solvent and the $\mathrm{pH}$ of a solution, strong fluctuations appear at large lag times, which results in a large deviation of the FCS curve as compared to the FCS curve resulting from a simple free diffusion of molecules at the TA probe. These fluctuations are probably caused by transient binding of molecules at the glass surface of the probe as demonstrated in corresponding FCS simulations obtained in this thesis.

As a conclusion, the theoretical and experimental results obtained in this thesis show the great potential of NSOM-FCS with the TA probe as a single molecule technique for spatially and temporally high-resolved dynamic measurements. The application on the translocation measurement at a biological membrane is a good evidence for this. A further system that could be well adapted for NSOM-FCS in the future is the giant unilamellar vesicle (GUV). These structures are model membranes that may enable us to study dynamic processes at a biological membrane in a much more fundamental way, since the required distance control for NSOM-FCS may be much easier achieved than at natural membranes. However, in order to use NSOM-FCS on GUVs or other samples, an adequate substrate has to be developed, so that the biological membrane is both free standing and well anchored so that it does not stick at the probe tip upon scanning.

In the field of plasmonics, NSOM-FCS may be of valuable help in order to better understand light-matter interaction at metal nanostructures. Also, time-gated NSOM-FCS measurements with pulsed excitation could be helpful to drive the signal-to-noise ratio of this technique at an even greater level. Further improvement of the signal-to-noise ratio would be certainly of great help to carry out a more systematic study on the probe's size dependence on the obsereved fluorescence enhancement, and this way may reveal a bunch of new information on the photophysical processes occuring at a nanoantenna. 


\section{A. Confined-diffusion model}

We consider the diffusion of a fluorescent particle captured in a one-dimensional channel with boundaries at $z=0$ and $L$ as depicted in figure 6.6a. If the particle is initially located at position $z$ than the probability density $p$ to find the particle after a time lag $\tau$ at the location $z^{\prime}$ is given by [65], [105]

$$
p\left(z, z^{\prime}, \tau\right)=\frac{1}{L} \sum_{m=-\infty}^{\infty} \exp \left(-D \tau\left(\frac{m \pi}{L}\right)^{2}\right) \cos (m \pi z / L) \cos \left(m \pi z^{\prime} / L\right)
$$

The fluorescence intensity $F$ of the particle is assumed to be proportional to the excitation intensity $I, F=Q I$. The normalized autocorrelation function $G(\tau)$ of $N$ particles is then given by

$$
G(\tau)=\frac{N}{L} \frac{Q^{2}}{\langle F\rangle^{2}} \int_{0}^{L} \mathrm{~d} z \int_{0}^{L} \mathrm{~d} z^{\prime} I(z) I\left(z^{\prime}\right) p\left(z, z^{\prime}, \tau\right)
$$

With $I(z)=I_{0} \exp (-z / d)$ it follows for the mean fluorescence intensity

$$
\langle F\rangle=Q I_{0} \frac{N}{L} \int_{0}^{L} \mathrm{~d} z \exp (-z / d)=Q I_{0} \frac{N d}{L}(1-\exp (-L / d))
$$

and thus

$$
\begin{aligned}
G(\tau)= & \frac{L}{N d^{2}}(1-\exp (-L / d))^{-2} \times \\
& \int_{0}^{L} \mathrm{~d} z \int_{0}^{L} \mathrm{~d} z^{\prime} \exp (-z / d) \exp \left(-z^{\prime} / d\right) p\left(z, z^{\prime}, \tau\right) \\
= & \frac{L}{N d^{2}}(1-\exp (-L / d))^{-2} \sum_{m=-\infty}^{\infty} \exp \left(-D \tau\left(\frac{m \pi}{L}\right)^{2}\right) \times \\
& \left\{\int_{0}^{L} \mathrm{~d} z \exp (-z / d) \cos (m \pi z / L)\right\}^{2}
\end{aligned}
$$


The solution of the integral is given by

$$
\int_{0}^{L} \mathrm{~d} z \exp (-z / d) \cos (m \pi z / L)=\frac{d}{1+(m \pi d / L)^{2}}\left(1-(-1)^{m} \exp (-L / d)\right)
$$

so that after a simplification due to the symmetry of the sum we arrive at

$$
G(\tau)=\frac{1}{N}\left\{1+2 \sum_{m=1}^{\infty} \frac{\exp \left(-\left(\frac{m \pi}{L}\right)^{2} D \tau\right)}{\left(1+\left(\frac{m \pi d}{L}\right)^{2}\right)^{2}}\left(\frac{1-(-1)^{m} \exp \left(-\frac{L}{d}\right)}{1-\exp \left(-\frac{L}{d}\right)}\right)^{2}\right\}
$$

So far, it was assumed that the particles are captured in the channel for an infinite time. By allowing particles to enter or leave the channel, we can treat the corresponding kinetics as a reversible bimolecular binding reaction between particles in solution and binding sites on a surface. The residence time $T_{R}$ in the channel is then equivalent to the inverse dissociation rate $\mathrm{kd}$ of the reaction. Supposing that the fluorescence of particles only occurs when associated to a binding site, here the channel, the autocorrelation function related to the binding kinetics of a single site is given by [73]

$$
G(\tau)=\frac{1}{C K} \exp \left(-\left(k_{a} C+k_{d}\right) \tau\right)
$$

with average concentration of particles in solution $C$, association and dissociation rate constants $k_{a}$ and $k_{d}$, respectively, and equilibrium constant $K=k_{a} / k_{d}$ of the reaction. Assuming that the fluorescence fluctuations arising from the binding kinetics are on a much slower time-scale than those of the diffusion in the channel, a separation of the dynamics is possible and the total autocorrelation function $G_{c d}(\tau)$ can be written as product of the single ones. Thus for a small enough concentration $C\left(k_{a} C \ll k_{d}\right)$ we finally get

$$
\begin{aligned}
G_{c d}(\tau) \propto \frac{1}{\langle N\rangle}\left\{1+2 \sum_{m=1}^{\infty} \frac{\exp \left(-\left(\frac{m \pi}{L}\right)^{2} D \tau\right)}{\left(1+\left(\frac{m \pi d}{L}\right)^{2}\right)^{2}}\left(\frac{1-(-1)^{m} \exp \left(-\frac{L}{d}\right)}{1-\exp \left(-\frac{L}{d}\right)}\right)^{2}\right\} \times \\
\quad \exp \left(-\frac{\tau}{T_{R}}\right)
\end{aligned}
$$




\section{Bibliography}

[1] E.B. Shera, N.K. Seitzinger, L.M. Davis, R.A. Keller, and S.A. Soper. Detection of single fluorescent molecules. Chem. Phys. Lett., 174:553-557, 1990. doi:10.1016/0009-2614(90)85485-U.

[2] S. Weiss. Fluorescence spectroscopy of single biomolecules. Science, 283:1676 1683, 1999. doi:10.1126/science.283.5408.1676.

[3] R. Kopelman and W. Tan. Near-field optics: Imaging single molecules. Science, 262:1382-1384, 1993. doi:10.1126/science.262.5138.1382.

[4] S. Nie, DT Chiu, and R.N. Zare. Probing individual molecules with confocal fluorescence microscopy. Science, 266:1018-1021, 1994. doi:10.1126/ science. 7973650 .

[5] E. Betzig and J.K. Trautman. Near-field optics: Microscopy, spectroscopy, and surface modification beyond the diffraction limit. Science, 257:189-195, 1992. doi:10.1126/science.257.5067.189.

[6] S.W. Hell and H.K. Stelzer. Fundamental improvement of resolution with a 4pi-confocal fluorescence microscope using two-photon excitation. Opt. Commun., 93:277-282, 1992. doi:10.1016/0030-4018(92)90185-T.

[7] M.G.L. Gustafsson. Surpassing the lateral resolution limit by a factor of two using structured illumination microscopy. J. Microsc., 198:82-87, 2000. doi:10.1046/j.1365-2818.2000.00710.x.

[8] S.W. Hell. Microscopy and its focal switch. Nat. Methods, 6:24-32, 2009. doi:10.1038/nmeth.1291.

[9] P.N. Hedde and G.U. Nienhaus. Optical imaging of nanoscale cellular structures. Biophys. J., 2:147-158, 2010. doi:10.1007/s12551-010-0037-0.

[10] S.W. Hell and J. Wichmann. Breaking the diffraction resolution limit by stimulated emission: stimulated-emission-depletion fluorescence microscopy. Opt. Lett., 19:780-782, 1994. doi:10.1364/0L.19.000780.

[11] E. Betzig, G.H. Patterson, R. Sougrat, O.W. Lindwasser, S. Olenych, J.S. Bonifacino, M.W. Davidson, J. Lippincott-Schwartz, and H.F. Hess. Imaging intracellular fluorescent proteins at nanometer resolution. Science, 313:16421645, 2006. doi:10.1126/science.1127344. 
[12] M.J. Rust, M. Bates, and X. Zhuang. Sub-diffraction-limit imaging by stochastic optical reconstruction microscopy (storm). Nat. Methods, 3:793796, 2006. doi:10.1038/nmeth929.

[13] S. van de Linde, A. Löschberger, T. Klein, M. Heidbreder, S. Wolter, M. Heilemann, and M. Sauer. Direct stochastic optical reconstruction microscopy with standard fluorescent probes. Nat. Photonics, 6:991-1009, 2011. doi:10.1038/nprot.2011.336.

[14] J.B. Pawley, editor. Handbook of Biological Confocal Microscopy. Springer Verlag, 2006.

[15] E. Fort and S. Gresillon. Surface enhanced fluorescence. J. Phys. D Appl. Phys., 41:013001, 2008. doi:10.1088/0022-3727/41/1/013001.

[16] H. Rigneault, J. Capoulade, J. Dintinger, J. Wenger, N. Bonod, E. Popov, T.W. Ebbesen, and P.-F. Lenne. Enhancement of single-molecule fluorescence detection in subwavelength apertures. Phys. Rev. Lett., 95:117401, 2005. doi:10.1103/PhysRevLett.95.117401.

[17] J. Wenger, D. Gérard, H. Aouani, and H. Rigneault. Nanoaperture-enhanced signal-to-noise ratio in fluorescence correlation spectroscopy. Anal. Chem., 81:834-839, 2008. doi:10.1021/ac8024015.

[18] D. Gérard, J. Wenger, N. Bonod, E. Popov, and H. Rigneault. Nanoapertureenhanced fluorescence: Towards higher detection rates with plasmonic metals. Phys. Rev. B, 77:045413, 2008. doi:10.1103/PhysRevB.77.045413.

[19] W.E. Moerner and M. Orrit. Illuminating single molecules in condensed matter. Science, 283:1670-1676, 1999. doi:10.1126/science.283.5408. 1670.

[20] D.E. Koppel. Statistical accuracy in fluorescence correlation spectroscopy. Phys. Rev. A, 10:1938-1945, 1974. doi:10.1103/PhysRevA.10.1938.

[21] N.L. Thompson, T.P. Burghardt, and D. Axelrod. Measuring surface dynamics of biomolecules by total internal reflection fluorescence with photobleaching recovery or correlation spectroscopy. Biophys. J., 33:435-454, 1981. doi:10.1016/S0006-3495(81)84905-3.

[22] L. Kastrup, H. Blom, C. Eggeling, and S.W. Hell. Fluorescence fluctuation spectroscopy in subdiffraction focal volumes. Phys. Rev. Lett., 94:178104, 2005. doi:10.1103/PhysRevLett.94.178104.

[23] M. Leutenegger, C. Eggeling, and S.W. Hell. Analytical description of sted microscopy performance. Opt. Express, 18:26417-26429, 2010. doi:10.1364/ OE. 18.026417. 
[24] M.J. Levene, J. Korlach, S.W. Turner, M. Foquet, H.G. Craighead, and W.W. Webb. Zero-mode waveguides for single-molecule analysis at high concentrations. Science, 299:682-686, 2003. doi:10.1126/science.1079700.

[25] D. Vobornik, D.S. Banks, Z. Lu, C. Fradin, R. Taylor, and L.J. Johnston. Fluorescence correlation spectroscopy with sub-diffraction-limited resolution using near-field optical probes. Appl. Phys. Lett., 93:163904-1-163904-3, 2008. doi:10.1063/1.2998602.

[26] C. Manzo, T.S. van Zanten, and M.F. Garcia-Parajo. Nanoscale fluorescence correlation spectroscopy on intact living cell membranes with nsom probes. Biophys. J., 100:L8-L10, 2011. doi:10.1016/j.bpj.2010.12.3690.

[27] Lukas Novotny. From near-field optics to optical antennas. Physics Today, 64:47-52, 2011. doi:10.1063/PT.3.1167.

[28] Edward Hutchinson Synge. A suggested model for extending microscopic resolution into the ultra-microscopic region. Philos. Mag., 6:356-362, 1928.

[29] Lukas Novotny and Bert Hecht. Principles of Nano-Optics. Cambridge University Press, 2006.

[30] Jean-Marie Vigoureux and Daniel Courjon. Detection of nonradiative fields in light of the heisenberg uncertainty principle and the rayleigh criterion. Appl. Optics, 31:3170-3177, 1992. doi:10.1364/A0.31.003170.

[31] Q. Wu, G.D. Feke, R.D. Grober, and L.P. Ghislain. Realization of numerical aperture 2.0 using a gallium phosphide solid immersion lens. Appl. Phys. Lett., 75:125537, 1999. doi:10.1063/1.125537.

[32] Dieter W Pohl. Optical stethoscopy: Image recording with resolution lambda/20. Appl. Phys. Lett., 44:651-653, 1984. doi:10.1063/1.94865.

[33] F. Keilmann and R. Hillenbrand. Near-field microscopy by elastic light scattering from a tip. Philos. T. Roy. Soc. A, 362:787-805, 2004. doi: 10.1098/rsta.2003.1347.

[34] L. Novotny and S.J. Stranick. Near-field optical microscopy and spectroscopy with pointed probes. Annu. Rev. Phys. Chem., 57:303-331, 2006. doi:10. 1146/annurev . physchem.56.092503.141236.

[35] R.C. Reddick, R.J. Warmack, and T.L. Ferrell. New form of scanning optical microscopy. Phys. Rev. B, 39:767-770, 1989. doi:10.1103/PhysRevB.39. 767.

[36] R. Reddick, R. Warmack, D.W. Chilcott, S.L. Sharp, and T.L. Ferrell. Photon scanning tunneling microscopy. Rev. Sci. Instrum., 61:3669, 1990. doi: 10.1063/1. 1141534 . 
[37] Eric Betzig, J.K. Trautman, T.D. Harris, J.S. Weiner, and R.L. Kostelar. Breaking the diffraction barrier: optical microscopy on a nanometric scale. Science, 251:1468-1470, 1991. doi:10.1126/science.251.5000.1468.

[38] J.A. Veerman, A.M. Otter, L. Kuipers, and N.F. van Hulst. High definition aperture probes for near-field optical microscopy fabricated by focused ion beam milling. Appl. Phys. Lett., 72:3115-3117, 1998. doi: $10.1063 / 1.121564$.

[39] A. Naber, D. Molenda, U.C. Fischer, H.J. Mass, C. Höppener, N. Lu, and H. Fuchs. Enhanced light confinement in a near-field optical probe with a triangular aperture. Phys. Rev. Lett., 89:210801, 2002. doi:10.1103/ PhysRevLett.89.210801.

[40] Jin Au Kong. Electromagnetic wave theory. John Wiley \& Sons, 1986.

[41] B. Hecht, B. Sick, U.P. Wild, and et al. Scanning near-field optical microscopy with aperture probes: Fundamentals and applications. J. Chem. Phys., 112:7761-7774, 2000. doi:10.1063/1.481382.

[42] D. Zeisel, S. Nettesheim, B. Dutoit, and R. Zenobi. Pulsed laser-induced desorption and optical imaging on a nanometer scale with scanning near-field microscopy using chemically etched fiber tips. Appl. Phys. Lett., 68:2491, 1996. doi:10.1063/1.115831.

[43] T. Saiki and K. Matsuda. Near-field optical fiber probe optimized for illumination-collection hybrid mode operation. Appl. Phys. Lett., 74:27732775, 1999. doi:10.1063/1.123307.

[44] R. Stöckle, C. Fokas, V. Deckert, R. Zenobi, B. Sick, B. Hecht, and U.P. Wild. High-quality near-field optical probes by tube etching. Appl. Phys. Lett., 75:160, 1999. doi:10.1063/1.124305.

[45] M. Ohtsu, editor. Progress in Nano-Electro-Optics III. Springer Verlag, 2005.

[46] D. Molenda, G. Colas des Francs, U.C. Fischer, N. Rau, and A. Naber. High-resolution mapping of the optical near-field components at a triangular nano-aperture. Opt. Express, 13:10688-10696, 2005. doi:10.1364/OPEX.13. 010688.

[47] L. Novotny and C. Hafner. Light propagation in a cylindrical waveguide with a complex, metallic, dielectric function. Phys. Rev. E, 50:4094-4106, 1994. doi:10.1103/PhysRevE.50.4094.

[48] T.W. Ebbesen, H.J. Lezec, H.F. Ghaemi, T. Thio, and P.A. Wolff. Extraordinary optical transmission through sub-wavelength hole arrays. Nature, 391:667-669, 1998. doi:10.1038/35570. 
[49] M.G. Velasco, P. Cassicy, and H. Xu. Extraordinary transmission of evanescent modes through a dielectric-filled nanowaveguide. Opt. Commun., 284:4805-4809, 2011. doi:10.1016/j.optcom.2011.06.006.

[50] K. Tanaka, G.W. Burr, T. Grosjean, T. Maletzky, and U.C. Fischer. Superfocussing in a metal-coated tetrahedral tip by dimensional reduction of surface-to edge-plasmon modes. Appl. Phys. B, 93:257-266, 2008. doi: $10.1007 / \mathrm{s} 00340-008-3147-7$.

[51] G.M. Hale and M.R. Querry. Optical constants of water in the 200-nm to 200- $\mu \mathrm{m}$ wavelength region. Appl. Optics, 12:555-563, 1973. doi:10.1364/ A0.12.000555.

[52] A. Otto. Excitation of nonradiative surface plasma waves in silver by the method of frustrated total reflection. Z. Phys., 216:398-410, 1968. doi: 10.1007/BF01391532.

[53] E. Kretschmann. Die bestimmung optischer konstanten von metallen durch anregung von oberflächenplasmaschwingungen. Z. Physik, 241:313-324, 1971. doi:10.1007/BF01395428.

[54] R.H. Ritchie, E.T. Arakawa, J.J. Cowan, and R.N. Hamm. Surface-plasmon resonance effect in grating diffraction. Phys. Rev. Lett., 21:1530-1533, 1968. doi:10.1103/PhysRevLett.21.1530.

[55] B. Hecht, H. Bielefeldt, L. Novotny, Y. Inouye, and D.W. Pohl. Local excitation, scattering, and interference of surface plasmons. Phys. Rev. Lett., 77:1889-1892, 1996. doi:10.1103/PhysRevLett.77.1889.

[56] P. Biagioni, J.-S. Huang, and B. Hecht. Nanoantennas for visible and infrared radiation. March 2011. arXiv:1103.1568.

[57] L.J. Sherry, R. Jin, C.A. Mirkin, C. Schatz, and R.P. Van Duyne. Localized surface plasmon resonance spectroscopy of single silver triangular nanoprisms. Nano Lett., 6:2060-2065, 2006. doi:10.1021/n1061286u.

[58] N. Félidj, J. Grand, G. Laurent, J. Aubard, G. Lévi, A. Hohenau, N. Galler, F.R. Aussenegg, and J.R. Krenn. Multipolar surface plasmon peaks on gold nanotriangles. J. Chem. Phys., 128:094702, 2008. doi:10.1063/1.2839273.

[59] L.Y. Wu, B.M. Ross, and L.P. Lee. Optical properties of the crescent-shaped nanohole antenna. Nano Lett., 9:1956-1961, 2009. doi:10.1021/n19001553.

[60] Website of ATTO-TEC GmbH, 2011. URL: https://www.atto-tec.com/.

[61] J. Widengren, U. Mets, and R. Rigler. Fluorescence correlation spectroscopy of triplet states in solution: a theoretical and experimental study. J. Phys. Chem.-US, 99:13368-13379, 1995. doi:10.1021/j100036a009. 
[62] J. Widengren, R. Rigler, and U. Mets. Triplet-state monitoring by fluorescence correlation spectroscopy. J. Fluoresc., 4:255-258, 1994. doi: 10.1007/BF01878460.

[63] K.H. Drexhage. Dye Lasers. Springer-Verlag, Berlin, 1973.

[64] P. Anger, P. Bharadwaj, and L. Novotny. Enhancement and quenching of single-molecule fluorescence. Phys. Rev. Lett., 96:113002, 2006. doi:10. 1103/PhysRevLett.96.113002.

[65] E.L. Elson and D. Magde. Fluorescence correlation spectroscopy. i. conceptual basis and theory. Biopolymers, 13:1-27, 1974. doi:10.1002/bip.1974. 360130102.

[66] J. Widengren and U. Mets. Single Molecule Detection in Solution, chapter Conceptual Basis of Fluorescence Correlation Spectroscopy and Related Techniques as Tools in Bioscience, pages 69-120. Wiley-VCH, 2002.

[67] F.A. Haight. Handbook of the Poisson Distribution. John Wiley \& Sons, 1967.

[68] L.E. Reichl. A Modern Course in Statistical Physics. John Wiley \& Sons, 1998.

[69] R. Roy, S. Hohng, and T. Ha. A practical guide to single-molecule fret. Nat. Methods, 5:507-516, 2008. doi:10.1038/NMETH. 1208.

[70] V. Buschmann, K.D. Weston, and M. Sauer. Spectroscopic study and evaluation of red-absorbing fluorescent dyes. Bioconjugate Chem., 14:195-204, 2003. doi:10.1021/bc025600x.

[71] T. Dertinger, V. Pacheco, I. von der Hocht, R. Hartmann, I. Gregor, and J. Enderlein. Two-focus fluorescence correlation spectroscopy: A new tool for accurate and absolute diffusion measurements. ChemPhysChem, 8:433443, 2007. doi:10.1002/cphc. 200600638.

[72] C.B. Müller, A. Loman, V. Pacheco, F. Koberling, D. Willbold, W. Richtering, and J. Enderlein. Precise measurement of diffusion by multi-color dual-focus fluorescence correlation spectroscopy. Europhys. Lett., 83:46001, 2008. doi:10.1209/0295-5075/83/46001.

[73] T.E. Starr and N.L. Thompson. Total internal reflection with fluorescence correlation spectroscopy: Combined surface reaction and solution diffusion. Biophys. J., 80:1575-1584, 2001. doi:10.1016/S0006-3495(01)76130-9.

[74] Nist digital library of mathematical functions. NIST Website, 2011. URL: http://dlmf.nist.gov/7.2. 
[75] H.A. Bethe. Theory of diffraction by small holes. Phys. Rev., 66:163-182, 1944. doi:10.1103/PhysRev.66.163.

[76] C.J. Bouwkamp. On bethe's theory of diffraction by small holes. Philips. Res. Rep., 5:321-332, 1950.

[77] J.D. Jackson. Classical electrodynamics. John Wiley \& Sons, 1999.

[78] G. Colas des Francs, D. Molenda, U.C. Fischer, and A. Naber. Enhanced light confinement in a triangular aperture: Experimental evidence and numerical calculations. Phys. Rev. B, 72:165111, 2005. doi:10.1103/PhysRevB.72. 165111.

[79] A.A. Tracton, editor. Coatings Technology Fundamentals, Testing, and Processing Techniques. Taylor \& Francis Group, 2007.

[80] M. Leutenegger, M. Gösch, A. Perentes, P. Hoffmann, O.J.F. Martin, and T. Lasser. Confining the sampling volume for fluorescence correlation spectroscopy using a sub-wavelength sized aperture. Opt. Express, 14:956-969, 2006. doi:10.1364/OPEX.14.000956.

[81] J. Pérez. Aufbau eines optischen rasternahfeldmikroskop für transmissionsund reflexionsmessungen. Diplom Thesis, Universität Karlsruhe (TH), Institut für Angewandte Physik, 2008.

[82] J. Wissler. Moleküldiffusion durch einzelne Kernporenkomplexe des Froscheies (Xenopus laevis): Untersuchung mit einer neuen Vorrichtung im optischen Nahfeld und Laser-Fluoreszenzkorrelationsspektroskopie. PhD thesis, Karlsruher Institutes für Technologie (KIT), Institut für Angewandte Physik, 2010 .

[83] K. Schätzel. New concepts in correlator design. Inst. Phys. Conf. Ser., 77:175$184,1985$.

[84] K. Schätzel, M. Drewel, and S. Stimac. Photon correlation measurements at large lag times: Improving statistical accuracy. J. Mod. Optic., 35:711-718, 1988. doi: $10.1080 / 09500348814550731$.

[85] E. Rittweger, K.Y. Han, S.E. Irvine, C. Eggeling, and S.W. Hell. Sted microscopy reveals crystal colour centres with nanometric resolution. Nat. Photonics, 3:144-147, 2009. doi:10.1038/nphoton.2009.2.

[86] N.L. Thompson and B.L. Steele. Total internal reflection with fluorescence correlation spectroscopy. Nat. Protoc., 2:878-890, 2007. doi:10.1038/ nprot.2007.110. 
[87] C.V. Kelly, B.A. Baird, and H.G. Craighead. An array of planar apertures for near-field fluorescence correlation spectroscopy. Biophys. J., 100:L34-L36, 2011. doi:10.1016/j.bpj.2011.02.034.

[88] J. Mütze, T. Ohrt, and P. Schwille. Fluorescence correlation spectroscopy in vivo. Laser Photonics Rev., 5:52-67, 2011. doi:10.1002/lpor. 200910041.

[89] M. Herrmann, N. Neuberth, J. Wissler, J. Pérez, D. Gradl, and A. Naber. Near-field optical study of protein transport kinetics at a single nuclear pore. Nano Lett., 9:3330-3336, 2009. doi:10.1021/n1901598z.

[90] M. Stewart. Molecular mechanism of the nuclear protein import cycle. Nat. Rev. Mol. Cell Biol., 8:195-208, 2007. doi:10.1038/nrm2114.

[91] L.J. Terry, E.B. Shows, and S.R. Wente. Crossing the nuclear envelope: Hierarchical regulation of nucleocytoplasmic transport. Science, 318:14121416, 2007. doi:10.1126/science.1142204.

[92] R.Y. Lim, U. Aebi, and B. Fahrenkrog. Towards reconciling structure and function in the nuclear pore complex. Histochem. Cell Biol., 129:105-116, 2008. doi:10.1007/s00418-007-0371-x.

[93] D. Stoffler, B. Feja, B. Fahrenkrog, J. Walz, D. Typke, and U. Aebi. Cryoelectron tomography provides novel insights into nuclear pore architecture: Implications for nucleocytoplasmic transport. J. Mol. Biol., 328:119-130, 2003. doi:10.1016/S0022-2836(03)00266-3.

[94] N. Elad, T. Maimon, D. Frenkiel-Krispin, R. Lim, and R.Y. Medalia. Structural analysis of the nuclear pore complex by integrated approaches. Curr. Opin. Struct. Biol., 19:226-232, 2009. doi:10.1016/j.sbi.2009.02.009.

[95] S.S. Patel, B.J. Belmont, J.M. Sante, and M.F. Rexach. Natively unfolded nucleoporins gate protein diffusion across the nuclear pore complex. Cell, 129:83-96, 2007. doi:10.1016/j.cell.2007.01.044.

[96] K. Ribbeck and D. Görlich. Kinetic analysis of translocation through nuclear pore complexes. EMBO J., 20:1320-1330, 2001. doi:10.1093/emboj/20.6. 1320 .

[97] K. Ribbeck, G. Lipowsky, H.M. Kent, M. Stewart, and D. Görlich. Ntf2 mediates nuclear import of ran. EMBO J., 17:6587-6598, 1998. doi:10. 1093/emboj/17.22.6587.

[98] A.J. García-Sáez and P. Schwille. Fluorescence correlation spectroscopy for the study of membrane dynamics and protein/lipid interactions. Methods, 46:116-122, 2008. doi:10.1016/j . ymeth.2008.06.011. 
[99] A. Mooradian. Photoluminescence of metals. Phys. Rev. Lett., 22:185-187, 1969. doi:10.1103/PhysRevLett.22.185.

[100] M. R. Beversluis, A. Bouhelier, and L. Novotny. Continuum generation from single gold nanostructures through near-field mediated intraband transitions. Phys. Rev. B, 68:115433, 2003. doi:10.1103/PhysRevB.68.115433.

[101] T.A. Laurence and S. Weiss. How to detect weak pairs. Science, 31:667-668, 2003. doi:10.1126/science.1081025.

[102] A. Penzkofer and Y. Lu. Fluorescence quenching of rhodamine $6 \mathrm{~g}$ in methanol at high concentration. Chem. Phys., 103:399-405, 1985. doi: 10.1016/0301-0104(86)80041-6.

[103] Life Technologies. The Molecular Probes Handbook - A Guide to Fluorescent Probes and Labeling Technologies. Life Technologies, 2010.

[104] P. Sandström, M. Boncheva, and B. Åkerman. Nonspecific and thiol-specific binding of dna to gold nanoparticles. Langmuir, 19:7537-7543, 2003. doi: 10.1021/la034348u.

[105] A. Gennerich and D. Schild. Fluorescence correlation spectroscopy in small cytosolic compartments depends critically on the diffusion model used. Biophys. J., 79:3294-3306, 2000. doi:10.1016/S0006-3495(00)76561-1. 



\section{Publications}

Parts of this work has already been published in scientific journals:

- M. Herrmann, N. Neuberth, J. Wissler, J. Pérez, D. Gradl, and A. Naber, "Near-Field Optical Study of Protein Transport Kinetics at a Single Nuclear Pore", Nano Lett. 9, 3330-3336 (2009)

- A. Naber, J. Wissler, J. Pérez, M. Herrmann, and D. Gradl, "Fluorescence correlation spectroscopy of particles in a transport channel illuminated by the evanescent field of a near-field optical aperture", Proc. of SPIE 7571, 757105 (2010)

- J. Pérez, M. Herrmann, D. Gradl, and A. Naber "Near-field optical fluorescence correlation spectroscopy", Proc. of SPIE 7571, 757118 (2010) 



\section{Acknowledgements}

In the first place, I would like to express my gratitude to Priv. Doz. Dr. Andreas Naber who gave me the opportunity to work on this such interdisciplinary field of research. It was a great pleasure to work together in this nice atmosphere. The discussions were always very fruitful. The only thing I regret is that the time available was a little too short to test all the new ideas that came up from these discussions. I also largely benefited from his great programming skills to facilitate this work. And for this I am very thankful. Last but not least, I appreciated a lot the several ideas he gave me in order to improve the writing of this thesis.

I am very indebted to Prof. Dr. Heinz Kalt, who kindly agreed to referee this thesis despite the request on short notice.

I am also thankful to Prof. Dr. Martin Wegener in the group of whom I was allowed to carry out my research. The availibility of excellent equipment in his group made a lot of experiments much easier.

I am very indebted to Patrice Brenner from the Laboratory for Electron Microscopy who operates the focus ion beam facility. His technical skills, his patience and flexibility for the many "aperture cutting" sessions were also greatly appreciated. With his participation the cutting of apertures in the metal coated near-field probes became a fast paced task carried out in a pleasant atmosphere.

The collaboration with Nicole Neuberth, Dr. Michael Herrmann, Dr. Dietmar Gradl and Dr. Jörg Wissler led to the successful biological application of NSOMFCS, for which I am grateful.

I would also like to thank the members of the group around Prof. Dr. Martin Wegener for the cordial atmosphere. Particularly, I thank Tolga Ergin for the simulation of the intensity distribution at the triangular aperture probe model.

Much of this work would not have been possible without the technical know-how of Heinz Hoffmann, Helmut Lay and Werner Gilde from the institute's electronic workshop and of the workers around Werner Wagner from the mechanical workshop. I am also very thankful for the great work realised by Thorsten Kuhn and Johann Westhauser, the former and current group's technician, respectively.

The Karlsruhe School of Optics and Photonics is greatly acknowledged for his financial support as well as for the many activities coordinated by Denica Angelova inside its $\mathrm{PhD}$ program.

Finally, I would like to thank my family for their support and patience. 
